\title{
Reproductive and Endocrine Biomarkers in Largemouth Bass (Micropterus salmoides) and Common Carp (Cyprinus carpio) from United States Waters
}

Steven L. Goodbred ${ }^{1}$, Stephen B. Smith ${ }^{2}$, Patricia S. Greene ${ }^{3}$, Richard H. Rauschenberger ${ }^{4}$, and Timothy M. Bartish ${ }^{5}$

${ }^{1}$ U.S. Geological Survey

Biological Resources Discipline

Sacramento, California

2 U.S. Geological Survey

Biological Resources Discipline

Reston, Virginia

${ }^{3}$ U.S. Geological Survey

Water Resources Discipline

Reston, Virginia

${ }^{4}$ U.S. Fish and Wildlife Service

Jacksonville, Florida

${ }^{5}$ U.S. Geological Survey

Biomonitoring of Environmental Status and Trends

Biological Resources Discipline

Ft. Collins, Colorado

Prepared in cooperation with the

U.S. Fish and Wild life Service

Data Series 2006-227

U.S. Department of the Interior

U.S. Geological Survey 


\section{U.S. Department of the Interior DIRK KEMPTHORNE, Secretary}

\section{U.S. Geological Survey Mark D. Myers, Director}

U.S. Geological Survey, Reston, Virginia: 2007

For product and ordering information:

World Wide Web: http://www.usgs.gov/pubprod

Telephone: 1-888-ASK-USGS

For more information on the USGS--the Federal source for science about the Earth, its natural and living resources, natural hazards, and the environment:

World Wide Web: http://www.usgs.gov

Telephone: 1-888-ASK-USGS

Any use of trade, product, or firm names is for descriptive purposes only and does not imply endorsement by the U.S. Government.

Although this report is in the public domain, permission must be secured from the individual copyright owners to reproduce any copyrighted materials contained within this report.

\section{Suggested Citation:}

Goodbred, S.L., Smith, S.B., Greene, P.S., Rauschenberger, R.H., Bartish, T.M., Reproductive and Endocrine Biomarkers in Largemouth Bass (Micropterus salmoides) and Common Carp (Cyprinus carpio) from United States Waters: U.S. Geological Survey Data Series 2006, 99 p.

This report can be accessed online at:

USGS Publications Warehouse-http://pubs.usgs.gov/ds/ds2006-227

and

USGS Florida Integrated Science Center-http://fisc.er.usgs.gov/Endocrine_Data_Report.pdf 


\section{Contents}

Abstract
Introduction.
Acknowledgments and Dedication
Materials and Methods
$\quad$ Sex Steroid Hormones
Vitellogenin
Results
References Cited

\section{Figure}

1. Map showing Regional boundaries and collection sites for common carp, largemouth bass, and both common carp and largemouth bass

\section{Tables}

1. Description of all study sites by region and species collected during 1994-97

2. National summary statistics (mean $\pm S E$ and ranges) for: fish size, biomarkers and reproductive stage for COMMON CARP and LARGEMOUTH BASS collected during non-reproductive (mid-August through March 1994-1997) and reproductive (April through mid-August 1995-1997) seasons

3. Site and regional mean values for biomarker and ancillary data for (A) COMMON CARP and (B) LARGEMOUTH BASS collections made at all sites in U.S. waters from March 1994 through August 1997

4. All individual COMMON CARP values for biomarker and ancillary data from collections made at sites in U.S. waters from August 1994 through October 1997

5. All individual LARGEMOUTH BASS values for biomarker and ancillary data from collections made at sites in U.S. waters from March 1994 through August 1997. 


\section{Selected Abbreviations and Acronyms}

${ }^{\circ} \mathrm{C}$ degree Celsius

ELISA Enzyme-Linked Immunosorbent Assay

g gram

$\mathrm{mL}$ milliliter

$\mathrm{mm}$ millimeter

$\mu \mathrm{g}$ microgram

$\mu \mathrm{L}$ microliter

ng nanogram

$\mathrm{nm}$ nanometer

$\mathrm{N}$ number of

pg picogram

$\mathrm{NaCl}$ sodium chloride

$\mathrm{NaPO}_{4}$ sodium phosphate

TBST tris-buffered saline

BEST Biomonitoring of Environmental Status and Trends Program (of the USGS)

CERC Columbia Environmental Research Center (of the USGS)

FISC Florida Integrated Science Center (of the USGS)

NAWQA National Water Quality Assessment Program (of the USGS)

USGS U.S. Geological Survey 


\title{
Reproductive and Endocrine Biomarkers in Largemouth Bass (Micropterus salmoides) and Common Carp (Cyprinus carpio) from United States Waters
}

\author{
By Steven L. Goodbred, Stephen B. Smith, Patricia S. Greene, Richard H. Rauschenberger, \\ and Timothy M. Bartish
}

\section{Abstract}

A nationwide reconnaissance investigation was initiated in 1994 to develop and evaluate a suite of reproductive and endocrine biomarkers for their potential to assess reproductive health and status in teleost (bony) fish. Fish collections were made at 119 sites, representing many regions of the country and land- and water-use settings. Collectively, this report will provide a national and regional benchmark and a basis for evaluating biomarkers of endocrine and reproductive function. Approximately 2,200 common carp (Cyprinus carpio) and 650 largemouth bass (Micropterus salmoides) were collected from 1994 through 1997. The suite of biomarkers used for these studies included: the plasma sex-steroid hormones, $17 \beta$-estradiol (E2) and 11 ketotestosterone (11KT); the ratio of E2 to $11 \mathrm{KT}$ (E2:11KT); plasma vitellogenin (VTG); and stage of gonadal development. This data report provides fish size, stage and reproductive biomarker data for individual fish and for site and regional summaries of these variables.

\section{Introduction}

Freshwater ecosystems have been shown to be the ultimate sink for many anthropogenic chemicals that can potentially affect aquatic communities and the terrestrial species that depend on them (Schmitt and others, 1999; Gross and others, 2003; Hoffman and others, 2003). Excess nutrients, runoff of pesticides from agricultural fields, industrial effluents, wastewater/sewage discharge, and urban nonpointsource contamination may contribute to the deterioration of freshwater aquatic environments. Many field studies designed to assess the effects of environmental contaminants in aquatic ecosystems focus efforts at local "hot spots," to evaluate the occurrence of endocrine disruption or the prevalence of external and internal anomalies (Baumann and others, 1991; Smith and others, 2002a,b). Although these focused studies have great value in determining cause-and-effect on select fish species at specific sites, a different approach is required to describe geographic differences, which is critical to determine if contaminant-induced effects might threaten populations of aquatic species nationally or regionally (Stahl and Clark, 1998). These data and the development of a battery of biomarkers designed to monitor the health and/or reproductive status of fish populations contribute to our understanding the well-being of aquatic resources, and help generate hypotheses concerning cause-and-effect relationships between environmental stressors (e.g., chemical contaminants) and fish health.

A variety of fish species are frequently used as sentinels or bioindicators for monitoring environmental quality in aquatic ecosystems. Teleost (bony fish) species are frequently preferred because of their wide geographic distribution, are relatively easy to sample, have a high reproductive rate, are integral components of the aquatic ecosystem, and are both commercially and recreationally important. Teleost fish in temperate regions undergo a seasonal reproductive cycle that has two distinct phases: (1) gonadal recrudescence/development, and (2) spawning. Cycling between these two phases is governed by both photoperiod and temperature, rainfall, water flow, as well as by other environmental cues (Kime, 1998; Gross and others, 2002, 2003). Most temperate fish in North America, such as black bass (Micropterus spp.), spawn during spring and early summer, but spawning time varies widely across a range of climatic conditions. Some species, such as common carp (Cyprinus carpio), may spawn as early as March in the southern U.S. to as late as mid-August in the northern U.S. (Panek, 1987). 
The two fish species selected for this study and described in this report, common carp and largemouth bass (Micropterus salmoides), are widely distributed across the United States. Although both species are oviparous (egg-laying), their reproductive strategies differ; common carp spawn by broadcasting eggs into the water column, whereas largemouth bass deposit eggs in nests. In addition, common carp are in the minnow family (Cyprinidae) and are omnivores, whereas largemouth bass are in the sunfish family (Centrarchidae) and are predators. Because they derive their food from different habitats, are in different trophic levels (Lee and others, 1980), and are in different families, they may also differ in their exposures to environmental contaminants at the same site and may have different physiological responses (Schmitt and others, 2002).

The biomarkers in this reconnaissance investigation were selected to measure a range of reproductively significant factors. The endocrine system is comprised of a wide variety of hormone-secreting cells, tissues, and ductless glands (e.g., the pituitary, thyroid, adrenal, and gonads), and each part plays an important role in growth, development, reproduction, and homeostasis of an organism. Endocrine hormones influence virtually every stage of the life cycle, from gametogenesis and fertilization, through development into a sexually mature organism. Other studies have reported correlations between specific impairment of reproduction (reduced fertility, hatchability and viability of offspring, impaired reproductive hormone activity, and/or altered sexual development and behavior) and elevated tissue concentrations of environmental contaminants (Hose and others, 1989; Tilitt and others 1992; Harrison and others, 1999; Gross and others, 2003). Abnormalities of these types may be caused by alteration of normal endocrine function either before or after hormone interactions with specific cellular receptors.

Concentrations of sex steroid hormones regulate important aspects of reproduction, such as spermatogenesis, follicular growth, and vitellogenesis. Initiation of these processes begins in the pituitary gland, which regulates gonadal hormone production in response to external cues (Kime, 1998; Gross and others, 2002, 2003). In most teleost fish, 17ßestradiol is the major estrogenic hormone in females responsible for ovarian growth. The concentration of 17ß-estradiol increases in the late winter to early spring, immediately preceding the spawning season, but its concentration is lower during the rest of the year (Norris and Jones, 1987; Down and others, 1990; Kime, 1998; Gross and others, 2002). In teleost fish, the primary sex steroid hormone in males is an androgen, 11-ketotestosterone, which is responsible for spermatogenesis (Borg, 1994; Mylonas and others, 1997; Cavaco and others, 1998; Cavaco and others, 1999). Concentrations of estrogens and androgens, and the ratio of two sex steroid hormones, $17 \beta$ estradiol/11-ketotestosterone (E2:11KT) have been utilized to assess the endocrine system in fish (Goodbred and others, 1997; Gross and others, 2002; Schmitt and others, 2002).

Vitellogenesis is the process of producing the protein vitellogenin in the liver in response to stimulation by estrogenic sex-steroid hormones. Vitellogenin is then processed by the

\section{Acknowledgments and Dedication}

This study was conducted by several U.S. Geological Survey (USGS) programs and research centers, including: the National Water Quality Assessment (NAWQA) Program, Biomonitoring of Environmental Status and Trends Program (BEST), the Florida Integrated Science Center (FISC), the Columbia Environmental Research Center (CERC), and personnel from both the Biological and the Water Resources disciplines. All biomarker analyses were conducted by the Joint Ecotoxicology Program of FISC and the University of Florida, College of Veterinary Medicine, Department of Physiological Sciences, and we wish to thank Tim Gross, Carla Wieser, Jon Wiebe, Nancy Denslow, and Kevin Kroll for these analyses. We also wish to thank members of NAWQA's biological team; Rachel Muir and Rod DeWeese for their field efforts and the review of this document and Wade Bryant for the expertise and field efforts. We also wish to thank the members of the BEST Program, Tim Bartish, Pat Anderson, and Jim Coyle for their expertise and support. In addition, we wish to acknowledge the critical support of the U.S. Fish and Wildlife Service in the field efforts nationally. The authors also want to thank all of the personnel from the various USGS NAWQA study units involved with the fish collections. Additional thanks to Steve Sorenson and Mike Yurewicz from the Northeast Region, and Christopher Schmitt from CERC.

Finally, and most importantly, we dedicate this report to our close friend and colleague, Tim Bartish, who is missed by all who knew him. 
ovary and transferred into developing follicles to form a large portion of the egg yolk. Male fish produce significant amounts of vitellogenin when exposed to estrogen or estrogenic chemicals (Purdom and others, 1994; Bromage and Cumaranatunga, 1998). Therefore, concentrations of vitellogenin have been recently recognized as an important biomarker for assessment of the reproductive status or health of fish (Denslow and others, 1999; Gross and others 2002, 2003; Schmitt and others, 2002). Vitellogenin provides an additional physiological biomarker for assessing reproductive health and predicting the ability of various compounds to alter the endocrine and reproductive systems. Males do not normally produce significant amounts of vitellogenin, but males treated with an estrogen produced significant vitellogenin concentrations. Therefore, it has been hypothesized that the elevated concentrations of plasma vitellogenin in males might serve as a reliable biomarker of exposure to environmental contaminants with estrogenic properties or endocrine effects (Heppell and others, 1995; Sumpter and Jobling, 1995; Palmer and Selcer, 1996; Denslow and others, 1996 a,b; 1997). Gonadal stage (stage of follicular or sperm maturation as determined by histological techniques) is also a potentially useful measure of the presence and effects of environmental stressors in teleost fish.
The purpose of this data report is to present multiple biomarkers of reproductive/endocrine function in common carp and largemouth bass on a national and regional scale in the United States.

\section{Materials and Methods Site Locations}

This report brings together data from sites sampled by a combination of two USGS monitoring/research efforts: (1) a national network of water-quality and bed-sediment and tissue sites of the USGS National Water Quality Assessment (NAWQA) Program (Gilliom and others, 1995); and (2) the sites in the Mississippi and Columbia River basins that were sampled by the USGS Biomonitoring of Environmental Status and Trends (BEST) Program (Schmitt and others, 1999; 2000).

The collection sites (fig. 1, table 1) represent a wide range of environmental settings and biologically important aquatic ecosystems across the contiguous United States; they are characterized by flow regime (riverine or lacustrine) and land use (agricultural, urban, or reference). The regional boundaries are rough estimates for the geographic separation of sites based on river basin and site proximity. There were no collection sites in the Southeast Region.

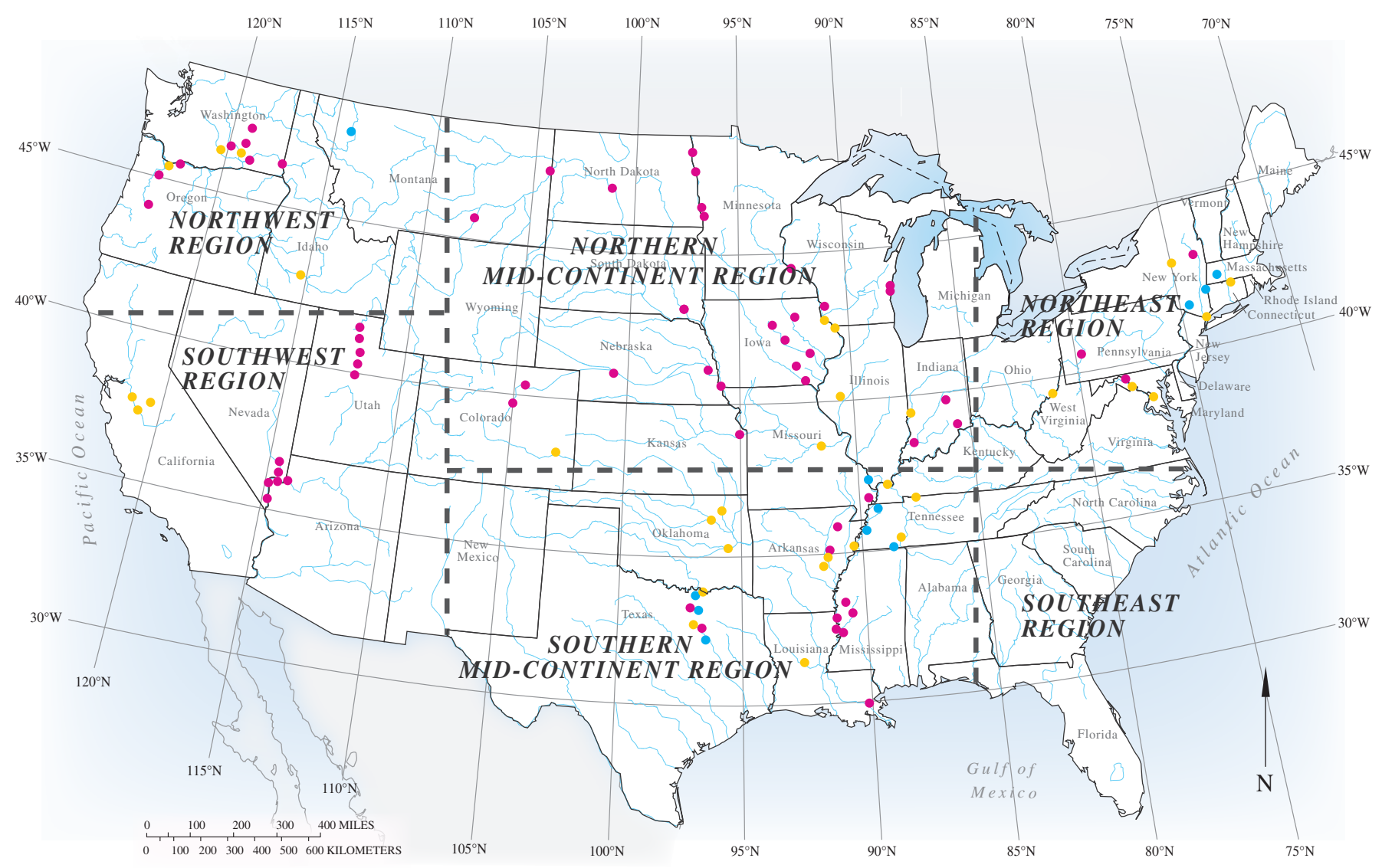

Figure 1. Regional boundaries and collection sites for common carp $\bullet$ (magenta), largemouth bass $\bullet$ (blue), and both common carp and largemouth bass $($ gold). 
Table 1. Description of all study sites by region and species collected during 1994-97.

\begin{tabular}{|c|c|c|c|c|c|c|c|c|c|c|}
\hline \multirow[b]{2}{*}{ Site name and location } & \multirow[b]{2}{*}{ Site code } & \multirow[b]{2}{*}{ Latitude } & \multirow[b]{2}{*}{ Longitude } & \multicolumn{2}{|c|}{ Flow regime } & \multicolumn{3}{|c|}{ Land use } & \multicolumn{2}{|c|}{ Species } \\
\hline & & & & Riverine & $\begin{array}{l}\text { Lacu- } \\
\text { strine }\end{array}$ & $\begin{array}{l}\text { Refer- } \\
\text { ence }\end{array}$ & $\begin{array}{l}\text { Agricul- } \\
\text { ture }\end{array}$ & Urban & $\begin{array}{c}\text { Common } \\
\text { carp }\end{array}$ & $\begin{array}{l}\text { Large- } \\
\text { mouth } \\
\text { bass }\end{array}$ \\
\hline
\end{tabular}

\section{Northeast Region}

\begin{tabular}{|c|c|c|c|c|c|c|c|c|c|c|}
\hline Connecticut Riv@ Thompsonville,CT & NECT-1 & $41^{\circ} 59^{\prime} \mathrm{N}$ & $72^{\circ} 36^{\prime} \mathrm{W}$ & $\mathrm{R}$ & & & & $\mathrm{U}$ & $\mathrm{C}$ & B \\
\hline Potomac Riv@ Anacostia Riv & NEDC-1 & $38^{\circ} 52^{\prime} \mathrm{N}$ & $76^{\circ} 59^{\prime} \mathrm{W}$ & $\mathrm{R}$ & & & & $\mathrm{U}$ & $\mathrm{C}$ & B \\
\hline Woods Pond at Lenox, MA & NEMA-1 & $42^{\circ} 21^{\prime} \mathrm{N}$ & $73^{\circ} 14^{\prime} \mathrm{W}$ & & $\mathrm{L}$ & & & $\mathrm{U}$ & & B \\
\hline Hudson Riv@L Lazume,NY & NENY-1 & $43^{\circ} 18^{\prime} \mathrm{N}$ & $73^{\circ} 50^{\prime} \mathrm{W}$ & $\mathrm{R}$ & & $\mathrm{R}$ & & & $\mathrm{C}$ & \\
\hline MohawkRiv@ Frankfort, NY & NENY-2 & $43^{\circ} 03^{\prime} \mathrm{N}$ & $75^{\circ} 04^{\prime} \mathrm{W}$ & $\mathrm{R}$ & & & & $\mathrm{U}$ & $\mathrm{C}$ & $\mathrm{B}$ \\
\hline Swinging Bridge Res nr Monticello, NY & NENY-3 & $41^{\circ} 37^{\prime} \mathrm{N}$ & $74^{\circ} 46^{\prime} \mathrm{W}$ & & $\mathrm{L}$ & $\mathrm{R}$ & & & & $\mathrm{B}$ \\
\hline Hudson Riv@ Poughkeepsie, NY & NENY-4 & $41^{\circ} 42^{\prime} \mathrm{N}$ & $73^{\circ} 55^{\prime} \mathrm{W}$ & $\mathrm{R}$ & & & & $\mathrm{U}$ & $\mathrm{C}$ & B \\
\hline Allegheny Riv@ Natrona,PA & NEPA-1 & $40^{\circ} 39^{\prime} \mathrm{N}$ & $79^{\circ} 41^{\prime} \mathrm{W}$ & $\mathrm{R}$ & & $\mathrm{R}$ & & & $\mathrm{C}$ & \\
\hline Shenandoah Riv @ Melville,wV & NEWV-1 & $39^{\circ} 16^{\prime} \mathrm{N}$ & $77^{\circ} 46^{\prime} \mathrm{W}$ & $\mathrm{R}$ & & & A & & $\mathrm{C}$ & \\
\hline Leetown Res at Leetown, WV & NEWV-2 & $39^{\circ} 23^{\prime} \mathrm{N}$ & $77^{\circ} 56^{\prime} \mathrm{W}$ & & $\mathrm{L}$ & $\mathrm{R}$ & & & $\mathrm{C}$ & B \\
\hline \multicolumn{11}{|l|}{ Northern Mid-Continent Region } \\
\hline Iowa Riv@ Morengo, IA & NMIA-1 & $41^{\circ} 50^{\prime} \mathrm{N}$ & $92^{\circ} 11^{\prime} \mathrm{W}$ & $\mathrm{R}$ & & & A & & $\mathrm{C}$ & \\
\hline South Fork at Iowa Riv @ Rowan, IA & NMIA-2 & $42^{\circ} 44^{\prime} \mathrm{N}$ & $93^{\circ} 35^{\prime} \mathrm{W}$ & $\mathrm{R}$ & & & A & & $\mathrm{C}$ & \\
\hline Iowa Riv nr New Providence, IA & NMIA-3 & $42^{\circ} 19^{\prime} \mathrm{N}$ & $93^{\circ} 09^{\prime} \mathrm{W}$ & $\mathrm{R}$ & & & A & & $\mathrm{C}$ & \\
\hline South Skunk River Oskaloosa, IA & NMIA-4 & $41^{\circ} 18^{\prime} \mathrm{N}$ & $92^{\circ} 38^{\prime} \mathrm{W}$ & $\mathrm{R}$ & & & A & & $\mathrm{C}$ & \\
\hline Cedar Riv@ @t. Chas City, IA & NMIA-5 & $43^{\circ} 03^{\prime} \mathrm{N}$ & $92^{\circ} 40^{\prime} \mathrm{W}$ & $\mathrm{R}$ & & & A & & $\mathrm{C}$ & \\
\hline Des Moines Riv@Keosauqua, IA & NMIA-6 & $40^{\circ} 44^{\prime} \mathrm{N}$ & $91^{\circ} 59^{\prime} \mathrm{W}$ & $\mathrm{R}$ & & & A & & $\mathrm{C}$ & \\
\hline Mississippi Riv @ Dubuque, IA & NMIA-7 & $42^{\circ} 26^{\prime} \mathrm{N}$ & $90^{\circ} 35^{\prime} \mathrm{W}$ & $\mathrm{R}$ & & & A & & $\mathrm{C}$ & B \\
\hline Mississippi Riv @ Guttenburg, IA & NMIA-8 & $42^{\circ} 43^{\prime} \mathrm{N}$ & $91^{\circ} 01^{\prime} \mathrm{W}$ & $\mathrm{R}$ & & & A & & $\mathrm{C}$ & $\mathrm{B}$ \\
\hline Illinois Riv @ Beardstown, IL & NMIL-1 & $40^{\circ} 07^{\prime} \mathrm{N}$ & $90^{\circ} 20^{\prime} \mathrm{W}$ & $\mathrm{R}$ & & & A & & $\mathrm{C}$ & $\mathrm{B}$ \\
\hline Sugar Creek nr New Palistine, IN & NMIN-1 & $39^{\circ} 43^{\prime} \mathrm{N}$ & $85^{\circ} 53^{\prime} \mathrm{W}$ & $\mathrm{R}$ & & & & $\mathrm{U}$ & $\mathrm{C}$ & \\
\hline Mississippi Riv@ L City, MN & NMMN-3 & $44^{\circ} 22^{\prime} \mathrm{N}$ & $92^{\circ} 07^{\prime} \mathrm{W}$ & & $\mathrm{L}$ & & & $\mathrm{U}$ & $\mathrm{C}$ & \\
\hline Missouri Riv@ Hermann, MO & NMMO-3 & $38^{\circ} 42^{\prime} \mathrm{N}$ & $91^{\circ} 26^{\prime} \mathrm{W}$ & $\mathrm{R}$ & & & A & & $\mathrm{C}$ & B \\
\hline Big Horn Riv @ Hardin, MT & NMMT-1 & $45^{\circ} 52^{\prime} \mathrm{N}$ & $107^{\circ} 34^{\prime} \mathrm{W}$ & $\mathrm{R}$ & & $\mathrm{R}$ & & & $\mathrm{C}$ & \\
\hline Yellowstone Riv @ Sidney, MT & NMMT-2 & $47^{\circ} 34^{\prime} \mathrm{N}$ & $104^{\circ} 13^{\prime} \mathrm{W}$ & $\mathrm{R}$ & & & A & & $\mathrm{C}$ & \\
\hline South Platte Riv below No Platte, NE & NMNE-1 & $41^{\circ} 08^{\prime} \mathrm{N}$ & $100^{\circ} 54^{\prime} \mathrm{W}$ & $\mathrm{R}$ & & & A & & $\mathrm{C}$ & \\
\hline Missouri Riv@ Nebraska City, NE & NMNE-3 & $40^{\circ} 40^{\prime} \mathrm{N}$ & $95^{\circ} 49^{\prime} \mathrm{W}$ & $\mathrm{R}$ & & & A & & $\mathrm{C}$ & \\
\hline RedRiv@ Drayton, ND & NMND-1 & $48^{\circ} 17^{\prime} \mathrm{N}$ & $97^{\circ} 12^{\prime} \mathrm{W}$ & $\mathrm{R}$ & & & A & & $\mathrm{C}$ & \\
\hline Red Riv nr Grand Forks, ND & NMND-2 & $47^{\circ} 54^{\prime} \mathrm{N}$ & $97^{\circ} 01^{\prime} \mathrm{W}$ & $\mathrm{R}$ & & & A & & $\mathrm{C}$ & \\
\hline Missouri Riv@ Garrison Dam, ND & NMND-3 & $47^{\circ} 28^{\prime} \mathrm{N}$ & $101^{\circ} 26^{\prime} \mathrm{W}$ & & $\mathrm{L}$ & $\mathrm{R}$ & & & $\mathrm{C}$ & \\
\hline James Riv@ Olivet, SD & NMSD-1 & $43^{\circ} 13^{\prime} \mathrm{N}$ & $97^{\circ} 41^{\prime} \mathrm{W}$ & $\mathrm{R}$ & & & A & & $\mathrm{C}$ & \\
\hline Mullet Riv@ Plymouth, WI & NMWI-1 & $43^{\circ} 46^{\prime} \mathrm{N}$ & $88^{\circ} 05^{\prime} \mathrm{W}$ & $\mathrm{R}$ & & & A & & $\mathrm{C}$ & \\
\hline North Br Milwaukee at Random Lake, WI & NMWI-2 & $43^{\circ} 02^{\prime} \mathrm{N}$ & $87^{\circ} 54^{\prime} \mathrm{W}$ & $\mathrm{R}$ & & & A & & $\mathrm{C}$ & \\
\hline Wisconsin Riv @ Woodman, WI & NMWI-3 & $43^{\circ} 05^{\prime} \mathrm{N}$ & $90^{\circ} 48^{\prime} \mathrm{W}$ & $\mathrm{R}$ & & $\mathrm{R}$ & & & $\mathrm{C}$ & \\
\hline
\end{tabular}


Table 1. (Continued) Description of all study sites by region and species collected during 1994-97.

\begin{tabular}{|c|c|c|c|c|c|c|c|c|c|c|}
\hline \multirow[b]{2}{*}{ Site name and location } & \multirow[b]{2}{*}{ Site code } & \multirow[b]{2}{*}{ Latitude } & \multirow[b]{2}{*}{ Longitude } & \multicolumn{2}{|c|}{ Flow regime } & \multicolumn{3}{|c|}{ Land use } & \multicolumn{2}{|c|}{ Species } \\
\hline & & & & Riverine & $\begin{array}{l}\text { Lacu- } \\
\text { strine }\end{array}$ & $\begin{array}{c}\text { Refer- } \\
\text { ence }\end{array}$ & $\begin{array}{l}\text { Agricul- } \\
\text { ture }\end{array}$ & Urban & $\begin{array}{l}\text { Common } \\
\text { carp }\end{array}$ & $\begin{array}{c}\text { Large- } \\
\text { mouth } \\
\text { bass }\end{array}$ \\
\hline \multicolumn{11}{|l|}{ Southern Mid-Continent Region } \\
\hline Cache Riv@ Cotton Plant, AR & SMAR-1 & $35^{\circ} 00^{\prime} \mathrm{N}$ & $91^{\circ} 11^{\prime} \mathrm{W}$ & $\mathrm{R}$ & & & A & & $\mathrm{C}$ & \\
\hline White River nr DeValls Bluff, AR & SMAR-3 & $34^{\circ} 44^{\prime} \mathrm{N}$ & $91^{\circ} 18^{\prime} \mathrm{W}$ & $\mathrm{R}$ & & & A & & $\mathrm{C}$ & \\
\hline Arkansas Riv@ Pine Bluff, AR & SMAR-4 & $34^{\circ} 16^{\prime} \mathrm{N}$ & $94^{\circ} 57^{\prime} \mathrm{W}$ & $\mathrm{R}$ & & & A & & $\mathrm{C}$ & B \\
\hline Ohio Riv@ Metropolis, IL & SMIL-2 & $37^{\circ} 07^{\prime} \mathrm{N}$ & $88^{\circ} 39^{\prime} \mathrm{W}$ & $\mathrm{R}$ & & & & $\mathrm{U}$ & $\mathrm{C}$ & B \\
\hline Tensas Riv@ Tendell, LA & SMLA-1 & $32^{\circ} 17^{\prime} \mathrm{N}$ & $91^{\circ} 07^{\prime} \mathrm{W}$ & $\mathrm{R}$ & & & A & & $\mathrm{C}$ & \\
\hline Mississippi Riv@ @uling, LA & SMLA-2 & $29^{\circ} 59^{\prime} \mathrm{N}$ & $90^{\circ} 25^{\prime} \mathrm{W}$ & $\mathrm{R}$ & & & & $\mathrm{U}$ & $\mathrm{C}$ & \\
\hline Big Sunflower Riv @ Anguilla, MS & SMMS-1 & $32^{\circ} 58^{\prime} \mathrm{N}$ & $90^{\circ} 50^{\prime} \mathrm{W}$ & $\mathrm{R}$ & & & A & & $\mathrm{C}$ & \\
\hline Boque Phalia at Leland, MS & SMMS-2 & $33^{\circ} 24^{\prime} \mathrm{N}$ & $90^{\circ} 54^{\prime} \mathrm{W}$ & $\mathrm{R}$ & & & A & & $\mathrm{C}$ & \\
\hline Steele Bayou at Rolling Fork, MS & SMMS-3 & $32^{\circ} 54^{\prime} \mathrm{N}$ & $90^{\circ} 52^{\prime} \mathrm{W}$ & $\mathrm{R}$ & & & A & & $\mathrm{C}$ & \\
\hline Yazoo Riv@ Redwood, MS & SMMS-4 & $32^{\circ} 55^{\prime} \mathrm{N}$ & $90^{\circ} 57^{\prime} \mathrm{W}$ & $\mathrm{R}$ & & & A & & $\mathrm{C}$ & \\
\hline Arkansas Riv @ Keystone Res, OK & SMOK-1 & $36^{\circ} 13^{\prime} \mathrm{N}$ & $96^{\circ} 15^{\prime} \mathrm{W}$ & & $\mathrm{L}$ & $\mathrm{R}$ & & & $\mathrm{C}$ & B \\
\hline Canadian Riv@Eufaula, OK & SMOK-2 & $35^{\circ} 16^{\prime} \mathrm{N}$ & $95^{\circ} 34^{\prime} \mathrm{W}$ & & $\mathrm{L}$ & & A & & $\mathrm{C}$ & B \\
\hline Verdigris Riv@ Oologah,OK & SMOK-3 & $36^{\circ} 31^{\prime} \mathrm{N}$ & $95^{\circ} 33^{\prime} \mathrm{W}$ & & $\mathrm{L}$ & & A & & $\mathrm{C}$ & B \\
\hline Wolf River nr LaGrange, TN & SMTN-1 & $35^{\circ} 02^{\prime} \mathrm{N}$ & $89^{\circ} 15^{\prime} \mathrm{W}$ & $\mathrm{R}$ & & & A & & & B \\
\hline Mississippi Riv@ Memphis, TN & SMTN-2 & $35^{\circ} 08^{\prime} \mathrm{N}$ & $90^{\circ} 03^{\prime} \mathrm{W}$ & $\mathrm{R}$ & & & & $\mathrm{U}$ & $\mathrm{C}$ & B \\
\hline Real Foot L NWR nr Union City, TN & SMTN-3 & $36^{\circ} 21^{\prime} \mathrm{N}$ & $89^{\circ} 58^{\prime} \mathrm{W}$ & & $\mathrm{L}$ & $\mathrm{R}$ & & & & B \\
\hline White Rock Lake, Dallas, TX & SMTX-5 & $32^{\circ} 50^{\prime} \mathrm{N}$ & $96^{\circ} 43^{\prime} \mathrm{W}$ & & $\mathrm{L}$ & & & $\mathrm{U}$ & & B \\
\hline Chambers L nr Corscama, TX & SMTX-6 & $32^{\circ} 13^{\prime} \mathrm{N}$ & $96^{\circ} 33^{\prime} \mathrm{W}$ & & $\mathrm{L}$ & & & $\mathrm{U}$ & & $\mathrm{B}$ \\
\hline RedRiv@L Texoma,TX & SMTX-7 & $33^{\circ} 52^{\prime} \mathrm{N}$ & $96^{\circ} 47^{\prime} \mathrm{W}$ & & $\mathrm{L}$ & & A & & $\mathrm{C}$ & B \\
\hline \multicolumn{11}{|l|}{ Northwest Region } \\
\hline Snake Riv@ Hagerman, ID & NWID-1 & $42^{\circ} 47^{\prime} \mathrm{N}$ & $114^{\circ} 56^{\prime} \mathrm{W}$ & $\mathrm{R}$ & & & A & & $\mathrm{C}$ & B \\
\hline Snake Riv@ Lewiston, ID & NWID-2 & $46^{\circ} 24^{\prime} \mathrm{N}$ & $117^{\circ} 02^{\prime} \mathrm{W}$ & $\mathrm{R}$ & & & A & & $\mathrm{C}$ & \\
\hline Flathead Riv@ Creston, MT & NWMT-1 & $48^{\circ} 09^{\prime} \mathrm{N}$ & $114^{\circ} 11^{\prime} \mathrm{W}$ & $\mathrm{R}$ & & & A & & & B \\
\hline Willamette Riv below Portland, OR & NWOR-1 & $45^{\circ} 28^{\prime} \mathrm{N}$ & $122^{\circ} 39^{\prime} \mathrm{W}$ & $\mathrm{R}$ & & & & $\mathrm{U}$ & $\mathrm{C}$ & B \\
\hline North Fork Willamette Riv @ Spgfield, OR & NWOR-2 & $44^{\circ} 07^{\prime} \mathrm{N}$ & $123^{\circ} 05^{\prime} \mathrm{W}$ & & $\mathrm{L}$ & & & $\mathrm{U}$ & $\mathrm{C}$ & \\
\hline ColumbiaRiv@Warrendale, OR & NWOR-3 & $45^{\circ} 38^{\prime} \mathrm{N}$ & $121^{\circ} 58^{\prime} \mathrm{W}$ & $\mathrm{R}$ & & & A & & $\mathrm{C}$ & \\
\hline Willamette Riv@ Oregon Cty, OR & NWOR-4 & $45^{\circ} 19^{\prime} \mathrm{N}$ & $122^{\circ} 39^{\prime} \mathrm{W}$ & $\mathrm{R}$ & & & A & & $\mathrm{C}$ & \\
\hline Billy Clapp L nr Stratford, WA & NWWA-1 & $47^{\circ} 16^{\prime} \mathrm{N}$ & $121^{\circ} 01^{\prime} \mathrm{W}$ & & $\mathrm{L}$ & $\mathrm{R}$ & & & $\mathrm{C}$ & \\
\hline Royal L nr Othello, WA & NWWA-2 & $46^{\circ} 54^{\prime} \mathrm{N}$ & $119^{\circ} 54^{\prime} \mathrm{W}$ & & $\mathrm{L}$ & & A & & $\mathrm{C}$ & \\
\hline Columbia Riv@ Pasco, WA & NWWA-3 & $46^{\circ} 31^{\prime} \mathrm{N}$ & $119^{\circ} 16^{\prime} \mathrm{W}$ & $\mathrm{R}$ & & & A & & $\mathrm{C}$ & B \\
\hline ColumbiaRiv@ Vernita Bg, WA & NWWA-4 & $46^{\circ} 37^{\prime} \mathrm{N}$ & $119^{\circ} 51^{\prime} \mathrm{W}$ & $\mathrm{R}$ & & & A & & $\mathrm{C}$ & \\
\hline Snake Riv @ Ice Harbor Dam, WA & NWWA-5 & $46^{\circ} 14^{\prime} \mathrm{N}$ & $118^{\circ} 53^{\prime} \mathrm{W}$ & $\mathrm{R}$ & & & A & & $\mathrm{C}$ & \\
\hline Yakima Riv@ Granger, WA & NWWA-6 & $46^{\circ} 20^{\prime} \mathrm{N}$ & $120^{\circ} 12^{\prime} \mathrm{W}$ & $\mathrm{R}$ & & & A & & $\mathrm{C}$ & B \\
\hline
\end{tabular}


Table 1. (Continued) Description of all study sites by region and species collected during 1994-97.

\begin{tabular}{|c|c|c|c|c|c|c|c|c|c|c|}
\hline Site name and location & Site code & Latitude & Longitude & Riverine & $\begin{array}{l}\text { Lacu- } \\
\text { strine }\end{array}$ & $\begin{array}{l}\text { Refer- } \\
\text { ence }\end{array}$ & $\begin{array}{l}\text { Agricul- } \\
\text { ture }\end{array}$ & Urban & $\begin{array}{c}\text { Common } \\
\text { carp }\end{array}$ & $\begin{array}{c}\text { Large- } \\
\text { mouth } \\
\text { bass }\end{array}$ \\
\hline \multicolumn{11}{|l|}{ Southwest Region } \\
\hline San Joaquin Riv@Salt Slough, CA & SWCA-2 & $37^{\circ} 34^{\prime} \mathrm{N}$ & $121^{\circ} 09^{\prime} \mathrm{W}$ & $\mathrm{R}$ & & & A & & $\mathrm{C}$ & B \\
\hline San Joaquin Riv@ Vernalis, CA & SWCA-3 & $37^{\circ} 36^{\prime} \mathrm{N}$ & $121^{\circ} 10^{\prime} \mathrm{W}$ & $\mathrm{R}$ & & & A & & $\mathrm{C}$ & B \\
\hline Virgin Riv inflo to L Mead, NV & SWNV-1 & $36^{\circ} 33^{\prime} \mathrm{N}$ & $114^{\circ} 20^{\prime} \mathrm{W}$ & $\mathrm{R}$ & & $\mathrm{R}$ & & & $\mathrm{C}$ & \\
\hline Muddy Riv inflo to L Mead, NV & SWNV-2 & $36^{\circ} 17^{\prime} \mathrm{N}$ & $114^{\circ} 30^{\prime} \mathrm{W}$ & $\mathrm{R}$ & & & A & & $\mathrm{C}$ & \\
\hline Las Vegas Bay nr Las Vegas Wash, NV & SWNV-3 & $36^{\circ} 06^{\prime} \mathrm{N}$ & $114^{\circ} 48^{\prime} \mathrm{W}$ & & $\mathrm{L}$ & & & $\mathrm{U}$ & $\mathrm{C}$ & \\
\hline Jordon Riv @ 17th Street, UT & SWUT-2 & $40^{\circ} 41^{\prime} \mathrm{N}$ & $112^{\circ} 06^{\prime} \mathrm{W}$ & $\mathrm{R}$ & & & & $\mathrm{U}$ & $\mathrm{C}$ & \\
\hline Jordon Riv@ Cudahy, UT & SWUT-3 & $40^{\circ} 39^{\prime} \mathrm{N}$ & $111^{\circ} 58^{\prime} \mathrm{W}$ & $\mathrm{R}$ & & & A & & $\mathrm{C}$ & \\
\hline Jordon Riv@Utah Lake, UT & SWUT-4 & $40^{\circ} 22^{\prime} \mathrm{N}$ & $111^{\circ} 54^{\prime} \mathrm{W}$ & $\mathrm{R}$ & & & A & & $\mathrm{C}$ & \\
\hline Weber Riv@ Ogden Bay, UT & SWUT-5 & $41^{\circ} 13^{\prime} \mathrm{N}$ & $112^{\circ} 40^{\prime} \mathrm{W}$ & $\mathrm{R}$ & & & & $\mathrm{U}$ & $\mathrm{C}$ & \\
\hline
\end{tabular}

\section{Sex Steroid Hormones}

Plasma samples from both species were analyzed for 17ß-estradiol and 11-ketotestosterone using radioimmunoassay procedures. Plasma samples $(50 \mu \mathrm{L})$ were extracted twice with 5-mL diethyl ether prior to radioimmunoassay analysis. Each sample was analyzed in duplicate for both $17 ß-$ estradiol and 11-ketotestosterone and corrected for extraction efficiencies of $92 \pm 2.8$ percent and $86 \pm 3.3$ percent, respectively. Standard curves were prepared in a buffer with known amounts of radioinert 17ß-estradiol or 11-ketotestosterone $(1,5,10,25,50,100,250,500$, and $1,000 \mathrm{pg})$. The minimum detectable concentration was $6.4 \mathrm{pg} / \mathrm{mL}$ for 173 -estradiol and $8.1 \mathrm{pg} / \mathrm{mL}$ for 11-ketotestosterone. Cross-reactivities with other steroids for the 17ß-estradiol antiserum were as follows: 11.2 percent for estrone, 1.7 percent for estriol, less than 1.0 percent for 17alpha-estradiol and androstenedione, and less than 0.1 percent for all other steroids examined. Cross-reactivities of the 11-ketotestosterone antiserum with other steroids were: 9.7 percent for testosterone, 3.7 percent for a-dihydrotestosterone, less than 1.0 percent for androstenedione, and less than 0.1 percent for all other steroids examined. A pooled sample (approximately $275 \mathrm{pg}$ of 173 -estradiol/mL and $220 \mathrm{pg} 11$-ketotestosterone/mL) was assayed serially in $10,20,30,40$, and $50 \mu \mathrm{L}$ volumes (final volume of $50 \mu \mathrm{L}$ with charcoal-stripped plasma). Further characterization of the assays involved measurement of known amounts $(1,2,5,10,25,50,100,250$, and 500 pg) of $17 ß$-estradiol or 11 - ketotestosterone in $50 \mu \mathrm{L}$ of charcoalstripped plasma. Values of $\mathrm{R}^{2}$ for correlations between actual and measured amounts during the calibration were 0.93 for $17 ß$-estradiol and 0.88 for 11 -ketotestosterone. Interassay and intraassay coefficients of variation were 7.3 and 9.5 percent, respectively, for plasma 17ß-estradiol; and 9.1 and 8.7 percent, respectively, for plasma 11-ketotestosterone (Bevans and others, 1996; Goodbred and others, 1997; Schmitt and Dethloff, 2000).

\section{Vitellogenin}

Vitellogenin concentrations in plasma of both species were assayed and quantified by capture Enzyme-Linked Immunosorbent Assay (ELISA) as previously described by Goodbred and others (1997) and summarized below. Initially, vitellogenin from bass was purified by ion-exchange chromatography (Denslow and others, 1999) for use in the standard. The monoclonal antibody Mab HL 1080 1C8-3C11 was used in the ELISA assay. This antibody reacts specifically to bass vitellogenin and not with other plasma proteins. Purified antibody was diluted to $10 \mu \mathrm{g} / \mathrm{mL}$ in phosphate-buffer saline (0.01 M NaPO4, $150 \mathrm{~mm} \mathrm{NaCl}, \mathrm{pH} 7.4)$ and coated onto 96-well microlitre plates $(50 \mu \mathrm{g} / \mathrm{mL}$ per well), and stored overnight at $4{ }^{\circ} \mathrm{C}$. Plates were washed with tris-buffered saline (TBST) buffer (10 mM Tris, $\mathrm{pH} \mathrm{7,} 150 \mathrm{mM} \mathrm{NaCl}$, $0.5 \%$ Tween), blocked with 0.1 percent bovine serum albumin in TBST for 2 hours at room temperature, and thoroughly 
washed again three times with TBST. Plasma samples were diluted from 1:100 to 1:5,000 in 0.1 percent bovine serum albumin in TBST and $50 \mu \mathrm{g}$ was added in duplicate to microtitre plate wells and incubated overnight. Standard curves were from 10 to $1,000 \mathrm{ng} / \mathrm{mL}$, and were constructed in male plasma at the same dilution as the sample to be analyzed. Of this solution, only $50 \mu \mathrm{g}$ were placed in the well. Although the assay itself is very sensitive, plasma samples must be diluted at least to 1:100 to eliminate interferences that affect the assay, thus making the assay sensitive to $0.001 \mathrm{mg} / \mathrm{mL}$. Male control plasma was made from composites of plasma from fish collected at an uncontaminated site, which was shown by Western Blot assay to have no vitellogenin. The next day, plates were washed with TBST, incubated with $50 \mu \mathrm{g}$ per well rabbit anti-vitellogenin polyclonal antibody UF114, produced and characterized by University of Florida, diluted to 1:500, and incubated for 2 hours at room temperature. This discloses the vitellogenin captured by the monoclonal antibody in the first step. The polyclonal antibody was, in turn, disclosed by a goat anti-rabbit immunoglobulin IgG linked to alkaline phosphatase and incubated for 2 hours at room temperature.

After a final series of washes with TBST, $100 \mu \mathrm{g}$ of p-nitro-phenyl-phosphate in carbonate buffer ( $\mathrm{pH}$ 9.6) was added to each well and incubated for 30 minutes. The intensity of yellow color that developed was quantified at $405 \mathrm{~nm}$ with an automated ELISA reader. Vitellogenin concentrations were calculated from standard curves after subtracting the small nonspecific color reaction (about $0.2 \mathrm{~A}, 405 \mathrm{~nm}$ ) with male control plasma. The ELISA assay used in this study can detect between 10 to $100 \mathrm{ng}$ of vitellogenin per well, resulting in a sensitivity of about $0.001 \mathrm{mg} / \mathrm{mL}$. Each ELISA assay included a positive control, which was plasma with a known vitellogenin concentration, to test for interassay and intraassay variation. The coefficient of variation was calculated for each duplicate sample and, if it exceeded 10 percent, samples were rerun. Standard curves fit by linear regression were used to calculate vitellogenin concentration, with $\mathrm{R}^{2}$ values usually between 0.95 and 0.99 (Denslow and others, 1996 a,b; 1997; Schmitt and Dethloff, 2000).

\section{Histological Determination of Sexual Maturation}

Samples of male and female gonads were taken, fixed in the field with Bouins' solution or 10 percent buffered formalin, and transferred to 100 percent ethanol in the laboratory prior to processing. Testes were cut longitudinally and ovaries were cut transversely. Samples were embedded in paraffin, sectioned to $5 \mu \mathrm{m}$, and stained with hematoxylin and eosin for histological evaluation. All tissue slides were evaluated for reproductive stage, anomalies, and gonadal parasites.

Gonads of female fish were classified according to four stages of sexual maturation, based on evaluation of histological slides. Ovaries containing mostly perinucleolar oocytes at various stages of pre-vitellogenic growth were classified as undeveloped (stage 0 ). Ovaries showing a mixture of both perinucleolar and cortical alveoli oocytes were classified as early-vitellogenic (stage 1). Ovaries classified as early-mid vitellogenic (stage 2 ) had some vitellogenic oocytes of various sizes and development, with few to moderate numbers of vitelline granules, and few fully developed oocytes. Late vitellogenisis was classified as stages 3 and 4, ovaries in which most oocytes were at or near maximum size and contained numerous, densely packed vitelline granules. Stage 4 were considered vitellogenic but post-ovulatory. Stage 5 females were post-vitellogenic.

Male gonads were classified according to three stages of sexual maturation. Undeveloped testes with no spermatogenesis were considered stage 0 . Testes that were classified as early spermatogenic (stage 1) had thick germinal epithelium, with diffuse pronounced proliferation and maturation of spermatozoa. Mid-spermatogenic (stage 2) testes had germinal epithelium of moderate thickness, with diffuse moderate proliferation and maturation of sperm. Testes classified as late spermatogenic (stage 3) had mostly thin germinal epithelium, with only scattered spermatogenic activity characteristic of full-grown testes and the latest stage of maturity (Goodbred and others, 1997; Schmitt and Dethloff, 2000).

\section{Results}

More than 2,200 common carp and nearly 650 bass were collected at over 100 sites nationally. This is a relatively large sample size, and data from all collected fish were utilized in the national summary. Common carp and largemouth bass were collected during both non-reproductive and reproductive seasons from 1994 though 1997. Largemouth bass were collected only from the northeastern region during the reproductive season.

Summary statistics are provided for fish size, biomarkers, and reproductive stage by species (table 2) and by region and site (tables $3 \mathrm{a}$ and $3 \mathrm{~b}$ ). Biomarkers and ancillary information for individual common carp and largemouth bass are listed in tables 4 and 5, respectively. This information may serve as a unique and useful set of baseline values for comparison and for planning future studies. 
Table 2. National summary statistics (mean \pm SE and ranges) for: fish size, biomarkers and reproductive stage for COMMON CARP and LARGEMOUTH BASS collected during non-reproductive (mid-August through March 1994-1997) and reproductive (April through mid-August 1995-1997) seasons.

[N, number of fish; E2, 17ß Estradiol; 11KT, 11 ketotestosterone; VTG, vitellogenin; mm, millimeter; g, grams; pg, picograms; mg, milligram; mL, milliliter; $\mathrm{ND}$, nondetected]

\begin{tabular}{|c|c|c|c|c|c|c|c|c|c|c|c|c|}
\hline $\begin{array}{l}\text { Length } \\
\text { (millimeter) }\end{array}$ & $\mathbf{N}$ & $\begin{array}{l}\text { Weight } \\
\text { (gram) }\end{array}$ & $\mathbf{N}$ & $\begin{array}{c}\mathrm{E}_{2} \\
\begin{array}{c}\text { (picogram per } \\
\text { milliliter) }\end{array}\end{array}$ & $\mathbf{N}$ & $\begin{array}{c}11 \mathrm{KT} \\
\text { (picogram per } \\
\text { milliliter) }\end{array}$ & $\mathbf{N}$ & $\begin{array}{l}\text { Ratio of } \\
E_{2} \text { to KT }\end{array}$ & $\mathbf{N}$ & $\begin{array}{c}\text { VTG } \\
\text { (milligram per } \\
\text { milliliter) }\end{array}$ & $\mathbf{N}$ & $\begin{array}{l}\text { Developmental } \\
\text { stage }\end{array}$ \\
\hline COMMON & & & & & & & & & & & & \\
\hline
\end{tabular}

\section{Female non-reproductive season}

\begin{tabular}{|c|c|c|c|c|c|c|c|c|c|c|c|c|}
\hline $\begin{array}{c}535 \pm 3.5 \\
(278-920)\end{array}$ & 819 & $\begin{array}{c}2380 \pm 50.4 \\
(288-10000)\end{array}$ & 819 & $\begin{array}{c}1479 \pm 44.4 \\
(15-11401)\end{array}$ & 823 & $\begin{array}{c}641 \pm 27.5 \\
(13-9066)\end{array}$ & 823 & $\begin{array}{c}3.8 \pm 0.15 \\
(0.1-40.6)\end{array}$ & 823 & $\begin{array}{l}12.6 \pm 0.51 \\
(N D-108)\end{array}$ & 821 & $\begin{array}{c}2.7 \pm 0.02 \\
(0-4)\end{array}$ \\
\hline
\end{tabular}

\section{Female reproductive season}

\begin{tabular}{|c|c|c|c|c|c|c|c|c|c|c|c|c|}
\hline $\begin{array}{c}515 \pm 6.3 \\
(332-742)\end{array}$ & 233 & $\begin{array}{c}2220 \pm 93.1 \\
(479-6300)\end{array}$ & 233 & $\begin{array}{l}1070 \pm 49.3 \\
(17-4142)\end{array}$ & 234 & $\begin{array}{r}659 \pm 49.4 \\
(45-5066)\end{array}$ & 234 & $\begin{array}{c}2.6 \pm 0.18 \\
(0.0-24.8)\end{array}$ & 234 & $\begin{array}{c}18.1 \pm 1.64 \\
(\mathrm{ND}-124.8)\end{array}$ & 234 & $\begin{array}{c}2.8 \pm 0.04 \\
(0-4)\end{array}$ \\
\hline
\end{tabular}

\section{Male non-reproductive season}

\begin{tabular}{|c|c|c|c|c|c|c|c|c|c|c|c|c|}
\hline $\begin{array}{c}507 \pm 2.8 \\
(278-779)\end{array}$ & 831 & $\begin{array}{c}1868 \pm 31.4 \\
(324-6407)\end{array}$ & 831 & $\begin{array}{l}502 \pm 15.8 \\
(17-4546)\end{array}$ & 833 & $\begin{array}{l}1232 \pm 42.1 \\
(61-9699)\end{array}$ & 833 & $\begin{array}{c}0.7 \pm 0.04 \\
(0.0-19.3)\end{array}$ & 833 & $\begin{array}{c}0.1 \pm 0.04 \\
(\mathrm{ND}-25.4)\end{array}$ & 830 & $\begin{array}{c}2.1 \pm 0.03 \\
(0-3)\end{array}$ \\
\hline \multicolumn{13}{|c|}{ Male reproductive season } \\
\hline $\begin{array}{c}501 \pm 5.4 \\
(297-763)\end{array}$ & 254 & $\begin{array}{c}1991 \pm 72.2 \\
(325-7300)\end{array}$ & 254 & $\begin{array}{l}408 \pm 22.1 \\
(51-2565)\end{array}$ & 255 & $\begin{array}{l}1000 \pm 63.8 \\
(74-6821)\end{array}$ & 255 & $\begin{array}{l}0.7 \pm 0.05 \\
(0.0-6.9)\end{array}$ & 255 & $\begin{array}{l}0.01 \pm 0.05 \\
(\mathrm{ND}-12.0)\end{array}$ & 255 & $\begin{array}{c}2.5 \pm 0.04 \\
(1-3)\end{array}$ \\
\hline
\end{tabular}

\section{LARGEMOUTH BASS}

\section{Female non-reproductive season}

\begin{tabular}{|c|c|c|c|c|c|c|c|c|c|c|c|c|}
\hline $\begin{array}{c}369 \pm 4.0 \\
(231-595)\end{array}$ & 323 & $\begin{array}{c}869 \pm 30.8 \\
(140-2920)\end{array}$ & 320 & $\begin{array}{l}717 \pm 40.1 \\
(29-6330)\end{array}$ & 335 & $\begin{array}{c}343 \pm 17.3 \\
(12-2203)\end{array}$ & 335 & $\begin{array}{c}3.3 \pm 0.20 \\
(0.0-27.8)\end{array}$ & 335 & $\begin{array}{c}1.3 \pm 0.38 \\
(\mathrm{ND}-77.9)\end{array}$ & 335 & $\begin{array}{c}1.7 \pm 0.04 \\
(0-3)\end{array}$ \\
\hline
\end{tabular}

\section{Female reproductive season}

\begin{tabular}{|c|c|c|c|c|c|c|c|c|c|c|c|c|}
\hline & 18 & $\begin{array}{c}872 \pm 97.1 \\
(350-1648)\end{array}$ & 18 & $\begin{array}{c}2710 \pm 606.2 \\
(384-9670)\end{array}$ & 18 & $\begin{array}{c}704 \pm 154.3 \\
(108-2306)\end{array}$ & 18 & $\begin{array}{c}4.6 \pm 0.68 \\
(1.6-12.4)\end{array}$ & 18 & $\begin{array}{c}4.8 \pm 0.84 \\
(\mathrm{ND}-12.4)\end{array}$ & 18 & $\begin{array}{l}3.2 \pm 0.15 \\
\quad(2-5)\end{array}$ \\
\hline
\end{tabular}

Male non-reproductive season

\begin{tabular}{|c|c|c|c|c|c|c|c|c|c|c|c|c|c|}
\hline $\begin{array}{c}338 \pm 3.2 \\
(226-515)\end{array}$ & 294 & $\begin{array}{c}616 \pm 19.5 \\
(114-1918)\end{array}$ & 283 & $\begin{array}{l}375 \pm 16.1 \\
(11-1823)\end{array}$ & 302 & $\begin{array}{c}703 \pm 41.8 \\
(15-7451)\end{array}$ & 302 & $\begin{array}{l}1.0 \pm 0.06 \\
(0.0-6.9)\end{array}$ & 302 & $\begin{array}{l}0.0 \pm 0.02 \\
(\mathrm{ND}-3.0)\end{array}$ & 302 & $\begin{array}{c}2.3 \pm 0.04 \\
(0-3)\end{array}$ & 302 \\
\hline
\end{tabular}

Male reproductive season

\begin{tabular}{|c|c|c|c|c|c|c|c|c|c|c|c|c|}
\hline $\begin{array}{c}305 \pm 7.8 \\
(276-386)\end{array}$ & 14 & $\begin{array}{l}450 \pm 43.0 \\
(321-938)\end{array}$ & 14 & $\begin{array}{l}781 \pm 120.2 \\
(280-1621)\end{array}$ & 14 & $\begin{array}{l}1195 \pm 115.2 \\
(410-1706)\end{array}$ & 14 & $\begin{array}{l}0.8 \pm 0.21 \\
(0.2-3.0)\end{array}$ & 14 & $\begin{array}{c}0.0 \pm 0.02 \\
(\mathrm{ND}-0.3)\end{array}$ & 14 & $\begin{array}{c}3.0 \pm 0.0 \\
(3-3)\end{array}$ \\
\hline
\end{tabular}


Table 3A. Site and regional mean values for biomarker and ancillary data for COMMON CARP collections made at all sites in U.S. waters from March 1994 through August 1997.

[N, number of fish; E2, 17ß Estradiol; 11KT, 11 ketotestosterone; VTG, vitellogenin; mm, millimeter; g, grams; pg, picograms; mg, milligram; mL, milliliter; ND, nondetected]

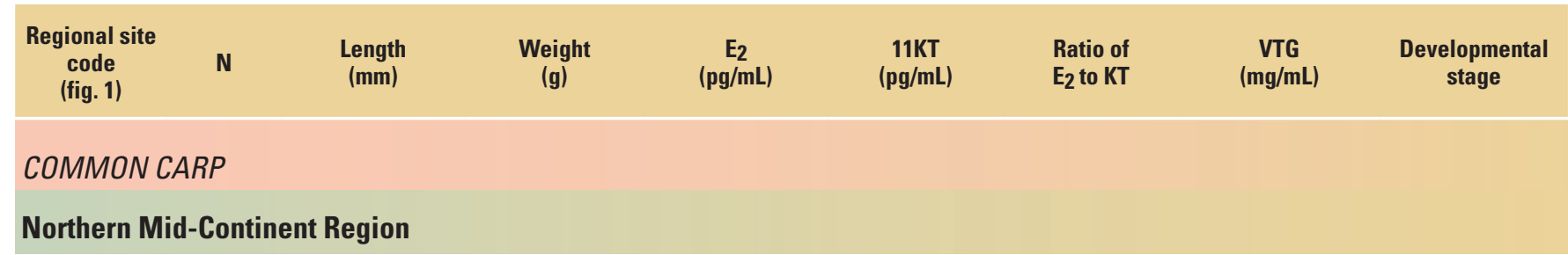

Female non-reproductive

\begin{tabular}{|c|c|c|c|c|c|c|c|c|}
\hline NMCO-1 & 32 & $\begin{array}{l}572 \pm 11.9 \\
(441-675)\end{array}$ & $\begin{array}{c}2827 \pm 178.7 \\
(1200-4588)\end{array}$ & $\begin{array}{c}1376 \pm 148.3 \\
(55-3249)\end{array}$ & $\begin{array}{l}668 \pm 69.2 \\
(127-1500)\end{array}$ & $\begin{array}{l}2 \pm 0.4 \\
(0-13)\end{array}$ & $\begin{array}{c}19 \pm 2.5 \\
(\mathrm{ND}-53.904)\end{array}$ & $\begin{array}{c}3 \pm 0.1 \\
(1-3)\end{array}$ \\
\hline NMCO-2 & 25 & $\begin{array}{l}533 \pm 7.5 \\
(480-620)\end{array}$ & $\begin{array}{l}2214 \pm 95.8 \\
(1559-3275)\end{array}$ & $\begin{array}{c}1519 \pm 112.4 \\
(559-2320)\end{array}$ & $\begin{array}{l}586 \pm 67.6 \\
(185-1250)\end{array}$ & $\begin{array}{c}3 \pm 0.2 \\
(2-6)\end{array}$ & $\begin{array}{c}29 \pm 1.4 \\
(14.7-45.9)\end{array}$ & $\begin{array}{l}3 \pm 0 \\
(3-3)\end{array}$ \\
\hline NMCO-3 & 11 & $\begin{array}{l}404 \pm 19.7 \\
(342-555)\end{array}$ & $\begin{array}{c}928 \pm 147.4 \\
(550-2150)\end{array}$ & $\begin{array}{c}967 \pm 154.6 \\
(366-1901)\end{array}$ & $\begin{array}{c}238 \pm 48.9 \\
(31-633)\end{array}$ & $\begin{array}{l}6 \pm 1.7 \\
(2-22)\end{array}$ & $\begin{array}{c}3 \pm 0.9 \\
(0.001-9.285)\end{array}$ & $\begin{array}{c}3 \pm 0.2 \\
(1-3)\end{array}$ \\
\hline NMIA-1 & 10 & $\begin{array}{c}461 \pm 18.8 \\
(360-530)\end{array}$ & $\begin{array}{c}1353 \pm 144.4 \\
(629-1970)\end{array}$ & $\begin{array}{c}1819 \pm 431.6 \\
(334-4819)\end{array}$ & $\begin{array}{c}303 \pm 42.2 \\
(57-569)\end{array}$ & $\begin{array}{l}6 \pm 1 \\
(2-13)\end{array}$ & $\begin{array}{c}3 \pm 0.5 \\
(0.181-5.132)\end{array}$ & $\begin{array}{c}3 \pm 0.2 \\
(1-3)\end{array}$ \\
\hline NMIA-2 & 10 & $\begin{array}{l}500 \pm 24.4 \\
(402-595)\end{array}$ & $\begin{array}{c}1928 \pm 284.5 \\
(970-3102)\end{array}$ & $\begin{array}{c}1578 \pm 312.1 \\
(143-3377)\end{array}$ & $\begin{array}{l}818 \pm 81.1 \\
(272-1155)\end{array}$ & $\begin{array}{c}2 \pm 0.7 \\
(0-8)\end{array}$ & $\begin{array}{c}4 \pm 0.6 \\
(\mathrm{ND}-6.93)\end{array}$ & $\begin{array}{c}3 \pm 0.1 \\
(2-3)\end{array}$ \\
\hline NMIA-3 & 3 & $\begin{array}{l}645 \pm 11.9 \\
(624-665)\end{array}$ & $\begin{array}{c}4166 \pm 280.3 \\
(3751-4700)\end{array}$ & $\begin{array}{c}1521 \pm 500.9 \\
(819-2491)\end{array}$ & $\begin{array}{c}250 \pm 117.5 \\
(112-484)\end{array}$ & $\begin{array}{c}7 \pm 0.9 \\
(5-8)\end{array}$ & $\begin{array}{c}3 \pm 0.4 \\
(2.736-4.159)\end{array}$ & $\begin{array}{l}3 \pm 0 \\
(3-3)\end{array}$ \\
\hline NMIA-4 & 10 & $\begin{array}{l}422 \pm 11.2 \\
(381-482)\end{array}$ & $\begin{array}{l}969 \pm 73.6 \\
(717-1355)\end{array}$ & $\begin{array}{c}1468 \pm 256.8 \\
(323-3276)\end{array}$ & $\begin{array}{c}287 \pm 25.4 \\
(121-397)\end{array}$ & $\begin{array}{c}6 \pm 1 \\
(1-11)\end{array}$ & $\begin{array}{c}3 \pm 0.4 \\
(0.027-4.999)\end{array}$ & $\begin{array}{c}3 \pm 0.2 \\
(1-3)\end{array}$ \\
\hline NMIA-5 & 10 & $\begin{array}{c}490 \pm 16.8 \\
(400-570)\end{array}$ & $\begin{array}{l}1534 \pm 159 \\
(811-2398)\end{array}$ & $\begin{array}{c}1846 \pm 202.9 \\
(1198-3190)\end{array}$ & $\begin{array}{c}571 \pm 108.7 \\
(135-1189)\end{array}$ & $\begin{array}{l}6 \pm 2.1 \\
(1-24)\end{array}$ & $\begin{array}{c}4 \pm 0.7 \\
(2.371-9.531)\end{array}$ & $\begin{array}{l}3 \pm 0 \\
(3-3)\end{array}$ \\
\hline NMIA-6 & 10 & $\begin{array}{l}538 \pm 14.3 \\
(462-605)\end{array}$ & $\begin{array}{l}2164 \pm 175 \\
(1310-2900)\end{array}$ & $\begin{array}{l}654 \pm 71.8 \\
(488-1220)\end{array}$ & $\begin{array}{l}280 \pm 45.2 \\
(132-522)\end{array}$ & $\begin{array}{c}3 \pm 0.4 \\
(1-5)\end{array}$ & $\begin{array}{c}2 \pm 0.5 \\
(0.045-5.482)\end{array}$ & $\begin{array}{c}3 \pm 0.2 \\
(1-3)\end{array}$ \\
\hline NMIA-7 & 10 & $\begin{array}{c}510 \pm 20.3 \\
(340-560)\end{array}$ & $\begin{array}{c}1964 \pm 181.8 \\
(555-2500)\end{array}$ & $\begin{array}{c}2262 \pm 353.8 \\
(1114-4852)\end{array}$ & $\begin{array}{l}714 \pm 75.4 \\
(351-1072)\end{array}$ & $\begin{array}{c}3 \pm 0.6 \\
(1-8)\end{array}$ & $\begin{array}{c}5 \pm 1.6 \\
(\mathrm{ND}-15.47)\end{array}$ & $\begin{array}{c}3 \pm 0.1 \\
(2-3)\end{array}$ \\
\hline NMIA-8 & 10 & $\begin{array}{l}483 \pm 7.5 \\
(441-513)\end{array}$ & $\begin{array}{l}1595 \pm 69.6 \\
(1230-1895)\end{array}$ & $\begin{array}{c}1576 \pm 276.7 \\
(639-3172)\end{array}$ & $\begin{array}{l}461 \pm 71.9 \\
(224-863)\end{array}$ & $\begin{array}{l}4 \pm 1.2 \\
(1-14)\end{array}$ & $\begin{array}{c}6 \pm 1.2 \\
(2.465-16.201)\end{array}$ & $\begin{array}{l}3 \pm 0 \\
(3-3)\end{array}$ \\
\hline NMIL-1 & 10 & $\begin{array}{c}421 \pm 15.6 \\
(366-529)\end{array}$ & $\begin{array}{c}1053 \pm 125.3 \\
(616-1864)\end{array}$ & $\begin{array}{l}548 \pm 92.2 \\
(171-1051)\end{array}$ & $\begin{array}{c}471 \pm 76.8 \\
(101-846)\end{array}$ & $\begin{array}{c}1 \pm 0.3 \\
(1-3)\end{array}$ & $\begin{array}{c}4 \pm 0.4 \\
(2.316-6.344)\end{array}$ & $\begin{array}{l}3 \pm 0 \\
(3-3)\end{array}$ \\
\hline NMIN-1 & 9 & $\begin{array}{c}627 \pm 23.3 \\
(524-742)\end{array}$ & $\begin{array}{c}3582 \pm 448.2 \\
(1775-5600)\end{array}$ & $\begin{array}{c}809 \pm 110.2 \\
(504-1423)\end{array}$ & $\begin{array}{l}810 \pm 97.9 \\
(383-1257)\end{array}$ & $\begin{array}{c}1 \pm 0.1 \\
(1-2)\end{array}$ & $\begin{array}{c}28 \pm 1.9 \\
(16.9-33.4)\end{array}$ & $\begin{array}{l}3 \pm 0 \\
(3-3)\end{array}$ \\
\hline NMIN-2 & 10 & $\begin{array}{c}633 \pm 35.8 \\
(510-855)\end{array}$ & $\begin{array}{c}3691 \pm 732.4 \\
(1610-8959)\end{array}$ & $\begin{array}{c}2367 \pm 274.9 \\
(828-3833)\end{array}$ & $\begin{array}{c}893 \pm 162.8 \\
(248-1890)\end{array}$ & $\begin{array}{c}3 \pm 0.7 \\
(1-9)\end{array}$ & $\begin{array}{c}18 \pm 1 \\
(12.911-22.7)\end{array}$ & $\begin{array}{c}3 \pm 0.2 \\
(2-3)\end{array}$ \\
\hline NMIN-3 & 17 & $\begin{array}{c}569 \pm 13.9 \\
(446-668)\end{array}$ & $\begin{array}{l}2722 \pm 202 \\
(1314-4530)\end{array}$ & $\begin{array}{l}1722 \pm 133 \\
(884-2799)\end{array}$ & $\begin{array}{c}1177 \pm 116.6 \\
(325-1969)\end{array}$ & $\begin{array}{c}2 \pm 0.3 \\
(1-5)\end{array}$ & $\begin{array}{c}30 \pm 1.2 \\
(21.4-41.1)\end{array}$ & $\begin{array}{l}3 \pm 0 \\
(3-3)\end{array}$ \\
\hline NMIN-4 & 9 & $\begin{array}{l}562 \pm 24.8 \\
(447-718)\end{array}$ & $\begin{array}{c}3008 \pm 542.7 \\
(1800-7181)\end{array}$ & $\begin{array}{c}570 \pm 65.4 \\
(351-920)\end{array}$ & $\begin{array}{c}109 \pm 11.1 \\
(69-181)\end{array}$ & $\begin{array}{l}6 \pm 0.9 \\
(3-12)\end{array}$ & $\begin{array}{c}3 \pm 0.7 \\
(0.001-7.453)\end{array}$ & $\begin{array}{c}2 \pm 0.4 \\
(0-3)\end{array}$ \\
\hline NMKS-1 & 10 & $\begin{array}{c}619 \pm 20.3 \\
(533-752)\end{array}$ & $\begin{array}{c}3238 \pm 374.1 \\
(2075-5450)\end{array}$ & $\begin{array}{c}934 \pm 109.9 \\
(443-1583)\end{array}$ & $\begin{array}{l}837 \pm 44.3 \\
(637-1070)\end{array}$ & $\begin{array}{c}1 \pm 0.1 \\
(0-2)\end{array}$ & $\begin{array}{c}2 \pm 0.4 \\
(\mathrm{ND}-3.761)\end{array}$ & $\begin{array}{c}3 \pm 0.2 \\
(1-3)\end{array}$ \\
\hline
\end{tabular}


Table 3A. (Continued) Site and regional mean values for biomarker and ancillary data for COMMON CARP collections made at all sites in U.S. waters from March 1994 through August 1997.

[N, number of fish; E2, 17ß Estradiol; 11KT, 11 ketotestosterone; VTG, vitellogenin; mm, millimeter; g, grams; pg, picograms; mg, milligram; mL, milliliter; $\mathrm{ND}$, nondetected]

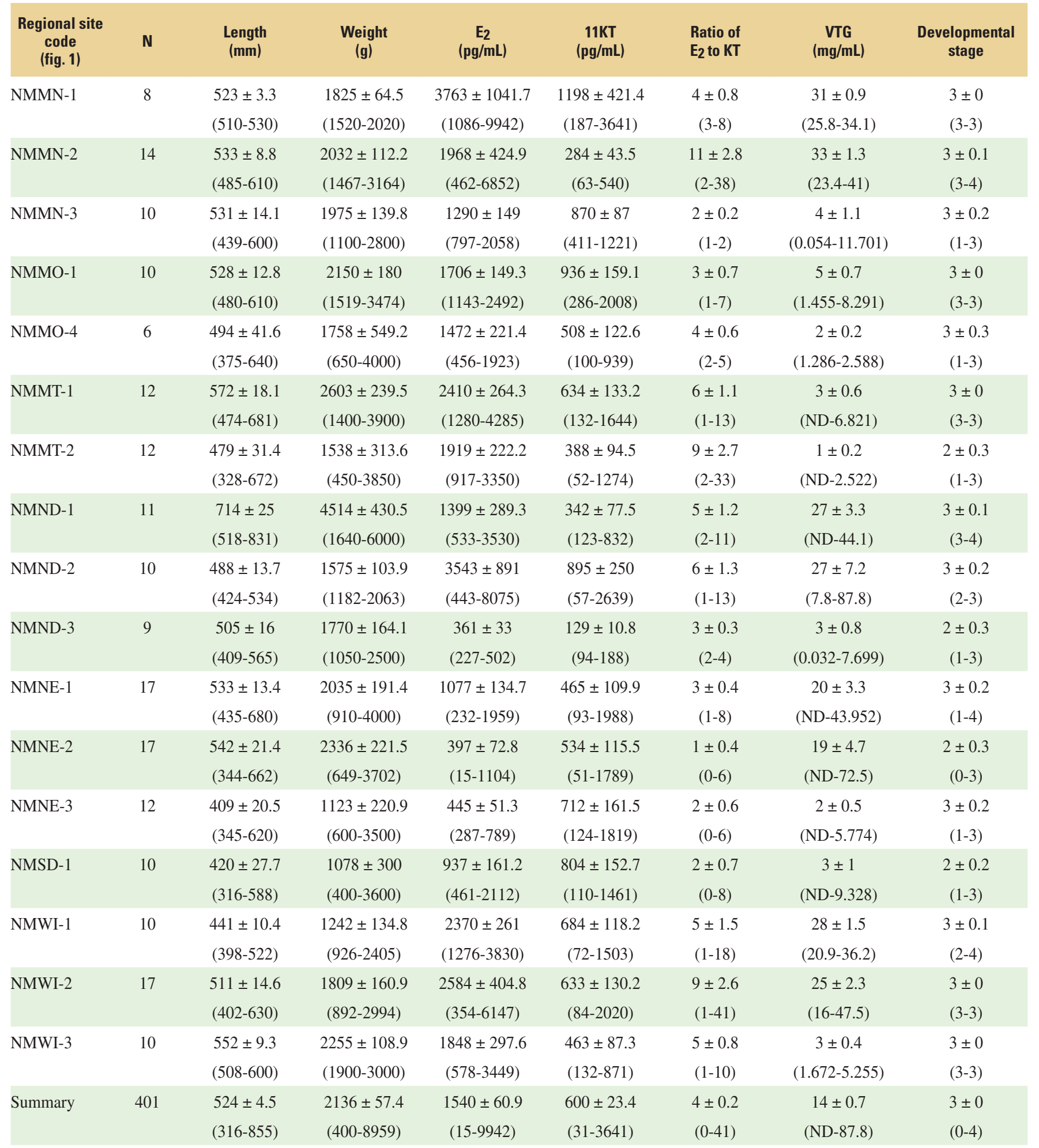


Table 3A. (Continued) Site and regional mean values for biomarker and ancillary data for COMMON CARP collections made at all sites in U.S. waters from March 1994 through August 1997.

[N, number of fish; E2, 17ß Estradiol; 11KT, 11 ketotestosterone; VTG, vitellogenin; mm, millimeter; g, grams; pg, picograms; mg, milligram; mL, milliliter; $\mathrm{ND}$, nondetected]

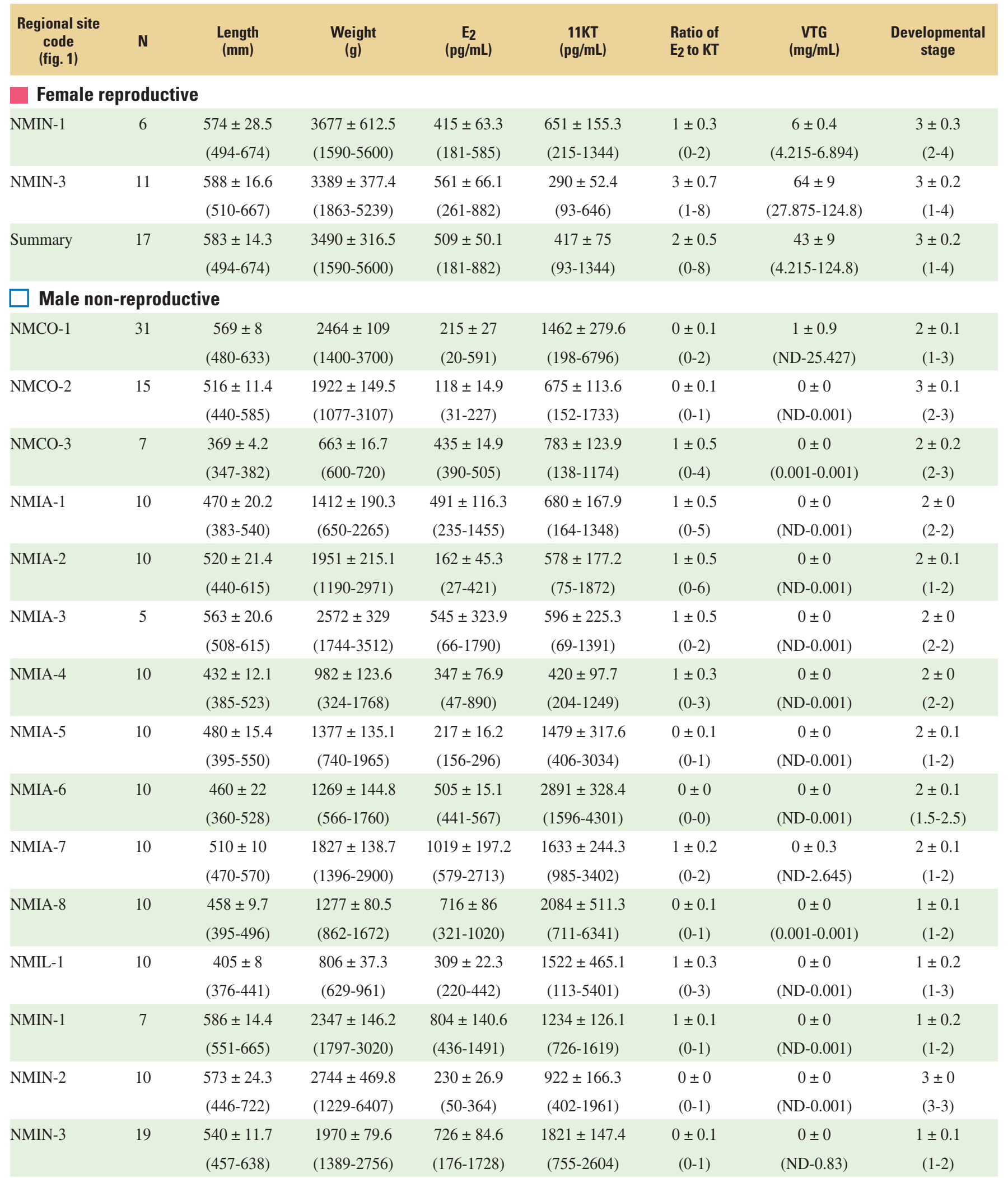




\section{Reproductive and Endocrine Biomarkers in Largemouth Bass and Common Carp from United States Waters}

Table 3A. (Continued) Site and regional mean values for biomarker and ancillary data for COMMON CARP collections made at all sites in U.S. waters from March 1994 through August 1997.

[N, number of fish; E2, 17ß Estradiol; 11KT, 11 ketotestosterone; VTG, vitellogenin; mm, millimeter; g, grams; pg, picograms; mg, milligram; mL, milliliter; $\mathrm{ND}$, nondetected]

\begin{tabular}{|c|c|c|c|c|c|c|c|c|}
\hline $\begin{array}{l}\text { Regional site } \\
\text { code } \\
\text { (fig. 1) }\end{array}$ & $\mathbf{N}$ & $\begin{array}{l}\text { Length } \\
\text { (mm) }\end{array}$ & $\begin{array}{l}\text { Weight } \\
\text { (g) }\end{array}$ & $\underset{(p g / m L)}{E_{2}}$ & $\begin{array}{c}11 \mathrm{KT} \\
(\mathrm{pg} / \mathrm{mL})\end{array}$ & $\begin{array}{l}\text { Ratio of } \\
E_{2} \text { to KT }\end{array}$ & $\underset{(\mathrm{mg} / \mathrm{mL})}{\mathrm{VTG}}$ & $\begin{array}{c}\text { Developmental } \\
\text { stage }\end{array}$ \\
\hline NMIN-4 & 8 & $\begin{array}{l}536 \pm 18.1 \\
(450-592)\end{array}$ & $\begin{array}{c}2275 \pm 181 \\
(1300-2800)\end{array}$ & $\begin{array}{l}411 \pm 26.7 \\
(310-544)\end{array}$ & $\begin{array}{c}858 \pm 261.2 \\
(246-2202)\end{array}$ & $\begin{array}{c}1 \pm 0.2 \\
(0-2)\end{array}$ & $\begin{array}{c}0 \pm 0 \\
(0.001-0.001)\end{array}$ & $\begin{array}{c}2 \pm 0.2 \\
(2-3)\end{array}$ \\
\hline NMKS-1 & 10 & $\begin{array}{c}573 \pm 15.8 \\
(460-621)\end{array}$ & $\begin{array}{c}2438 \pm 172.1 \\
(1200-2950)\end{array}$ & $\begin{array}{l}702 \pm 108.2 \\
(417-1445)\end{array}$ & $\begin{array}{l}1022 \pm 39.1 \\
(828-1206)\end{array}$ & $\begin{array}{c}1 \pm 0.1 \\
(0-2)\end{array}$ & $\begin{array}{c}0 \pm 0 \\
(\mathrm{ND}-0.013)\end{array}$ & $\begin{array}{c}2 \pm 0.2 \\
(1-3)\end{array}$ \\
\hline NMMN-1 & 9 & $\begin{array}{l}490 \pm 6.1 \\
(460-520)\end{array}$ & $\begin{array}{l}1435 \pm 58.9 \\
(1253-1763)\end{array}$ & $\begin{array}{c}586 \pm 141.5 \\
(57-1105)\end{array}$ & $\begin{array}{c}1174 \pm 339.3 \\
(77-2935)\end{array}$ & $\begin{array}{c}1 \pm 0.1 \\
(0-1)\end{array}$ & $\begin{array}{c}0 \pm 0 \\
(\mathrm{ND}-0.09)\end{array}$ & $\begin{array}{c}3 \pm 0.1 \\
(2-3)\end{array}$ \\
\hline NMMN-2 & 10 & $\begin{array}{c}505 \pm 15.4 \\
(450-620)\end{array}$ & $\begin{array}{l}1604 \pm 170 \\
(998-2918)\end{array}$ & $\begin{array}{c}759 \pm 118.2 \\
(267-1573)\end{array}$ & $\begin{array}{c}822 \pm 258.2 \\
(105-2261)\end{array}$ & $\begin{array}{c}2 \pm 0.6 \\
(0-7)\end{array}$ & $\begin{array}{c}0 \pm 0 \\
(\mathrm{ND}-0.05)\end{array}$ & $\begin{array}{l}3 \pm 0 \\
(3-3)\end{array}$ \\
\hline NMMN-3 & 10 & $\begin{array}{l}504 \pm 6.1 \\
(470-536)\end{array}$ & $\begin{array}{c}1975 \pm 298.5 \\
(1450-4600)\end{array}$ & $\begin{array}{l}862 \pm 78.7 \\
(445-1258)\end{array}$ & $\begin{array}{l}1457 \pm 111.5 \\
(1050-1963)\end{array}$ & $\begin{array}{c}1 \pm 0.1 \\
(0-1)\end{array}$ & $\begin{array}{c}0 \pm 0 \\
(\mathrm{ND}-0.008)\end{array}$ & $\begin{array}{l}1 \pm 0.1 \\
(1-1.5)\end{array}$ \\
\hline NMMO-1 & 10 & $\begin{array}{c}487 \pm 14 \\
(410-560)\end{array}$ & $\begin{array}{c}1590 \pm 132.4 \\
(1158-2467)\end{array}$ & $\begin{array}{c}429 \pm 135.2 \\
(17-1457)\end{array}$ & $\begin{array}{l}772 \pm 92.4 \\
(287-1389)\end{array}$ & $\begin{array}{c}1 \pm 0.2 \\
(0-2)\end{array}$ & $\begin{array}{c}0 \pm 0 \\
(\mathrm{ND}-0.001)\end{array}$ & $\begin{array}{c}2 \pm 0.1 \\
(2-3)\end{array}$ \\
\hline NMMO-4 & 9 & $\begin{array}{c}524 \pm 30.4 \\
(346-610)\end{array}$ & $\begin{array}{c}2078 \pm 287.4 \\
(550-3050)\end{array}$ & $\begin{array}{c}915 \pm 107.9 \\
(413-1431)\end{array}$ & $\begin{array}{c}1240 \pm 302.3 \\
(72-2954)\end{array}$ & $\begin{array}{c}3 \pm 2.1 \\
(0-19)\end{array}$ & $\begin{array}{c}0 \pm 0 \\
(\mathrm{ND}-0.001)\end{array}$ & $\begin{array}{l}2 \pm 0.2 \\
(1-2.5)\end{array}$ \\
\hline NMMT-1 & 8 & $\begin{array}{c}529 \pm 15.6 \\
(470-585)\end{array}$ & $\begin{array}{c}1775 \pm 161.8 \\
(1250-2600)\end{array}$ & $\begin{array}{c}1096 \pm 86.8 \\
(716-1418)\end{array}$ & $\begin{array}{c}2267 \pm 245 \\
(1184-2970)\end{array}$ & $\begin{array}{c}1 \pm 0.1 \\
(0-1)\end{array}$ & $\begin{array}{c}0 \pm 0.1 \\
(\mathrm{ND}-0.958)\end{array}$ & $\begin{array}{c}2 \pm 0.2 \\
(1-3)\end{array}$ \\
\hline NMMT-2 & 8 & $\begin{array}{c}551 \pm 26.3 \\
(390-619)\end{array}$ & $\begin{array}{c}2150 \pm 220.6 \\
(800-2800)\end{array}$ & $\begin{array}{c}1209 \pm 255.8 \\
(709-2918)\end{array}$ & $\begin{array}{c}2709 \pm 371.8 \\
(1191-4214)\end{array}$ & $\begin{array}{c}0 \pm 0.1 \\
(0-1)\end{array}$ & $\begin{array}{c}0 \pm 0 \\
(\mathrm{ND}-0.001)\end{array}$ & $\begin{array}{c}1 \pm 0.2 \\
(1-2)\end{array}$ \\
\hline NMND-1 & 10 & $\begin{array}{c}589 \pm 16.6 \\
(520-675)\end{array}$ & $\begin{array}{c}2352 \pm 203 \\
(1645-3470)\end{array}$ & $\begin{array}{l}444 \pm 95 \\
(100-901)\end{array}$ & $\begin{array}{c}965 \pm 129 \\
(508-1947)\end{array}$ & $\begin{array}{c}1 \pm 0.1 \\
(0-1)\end{array}$ & $\begin{array}{l}1 \pm 0.5 \\
(\mathrm{ND}-5)\end{array}$ & $\begin{array}{c}3 \pm 0.2 \\
(2-3)\end{array}$ \\
\hline NMND-2 & 4 & $\begin{array}{c}471 \pm 15.3 \\
(436-510)\end{array}$ & $\begin{array}{l}1252 \pm 83.4 \\
(1079-1470)\end{array}$ & $\begin{array}{c}1431 \pm 348.4 \\
(465-2104)\end{array}$ & $\begin{array}{c}857 \pm 238.1 \\
(387-1507)\end{array}$ & $\begin{array}{c}2 \pm 0.8 \\
(1-4)\end{array}$ & $\begin{array}{c}0 \pm 0 \\
(\mathrm{ND}-0.07)\end{array}$ & $\begin{array}{c}3 \pm 0.3 \\
(2-3)\end{array}$ \\
\hline NMND-3 & 11 & $\begin{array}{c}489 \pm 23.1 \\
(299-595)\end{array}$ & $\begin{array}{c}1625 \pm 226.6 \\
(420-3400)\end{array}$ & $\begin{array}{l}300 \pm 13 \\
(219-362)\end{array}$ & $\begin{array}{c}2249 \pm 513.2 \\
(887-6760)\end{array}$ & $\begin{array}{l}0 \pm 0 \\
(0-0)\end{array}$ & $\begin{array}{c}0 \pm 0 \\
(\mathrm{ND}-0.007)\end{array}$ & $\begin{array}{c}1 \pm 0.2 \\
(0-2)\end{array}$ \\
\hline NMNE-1 & 20 & $\begin{array}{l}509 \pm 11.1 \\
(390-615)\end{array}$ & $\begin{array}{c}1752 \pm 88.3 \\
(838-2350)\end{array}$ & $\begin{array}{c}308 \pm 54.3 \\
(39-881)\end{array}$ & $\begin{array}{c}1432 \pm 327.9 \\
(111-5303)\end{array}$ & $\begin{array}{c}0 \pm 0.1 \\
(0-2)\end{array}$ & $\begin{array}{c}1 \pm 0.5 \\
(\mathrm{ND}-10.981)\end{array}$ & $\begin{array}{c}1 \pm 0.1 \\
(1-2)\end{array}$ \\
\hline NMNE-2 & 18 & $\begin{array}{l}462 \pm 20 \\
(317-586)\end{array}$ & $\begin{array}{l}1331 \pm 137 \\
(464-2456)\end{array}$ & $\begin{array}{c}294 \pm 83.5 \\
(17-1171)\end{array}$ & $\begin{array}{c}600 \pm 123.1 \\
(76-2007)\end{array}$ & $\begin{array}{c}1 \pm 0.2 \\
(0-2)\end{array}$ & $\begin{array}{c}0 \pm 0 \\
(\mathrm{ND}-0.01)\end{array}$ & $\begin{array}{c}2 \pm 0.3 \\
(0-3)\end{array}$ \\
\hline NMNE-3 & 11 & $\begin{array}{c}472 \pm 34.5 \\
(352-680)\end{array}$ & $\begin{array}{l}1655 \pm 331 \\
(600-3450)\end{array}$ & $\begin{array}{c}363 \pm 18.8 \\
(283-482)\end{array}$ & $\begin{array}{c}2205 \pm 218.5 \\
(1142-3136)\end{array}$ & $\begin{array}{l}0 \pm 0 \\
(0-0)\end{array}$ & $\begin{array}{c}0 \pm 0 \\
(\mathrm{ND}-0.001)\end{array}$ & $\begin{array}{c}1 \pm 0.1 \\
(1-2)\end{array}$ \\
\hline NMSD-1 & 9 & $\begin{array}{c}494 \pm 32.1 \\
(319-603)\end{array}$ & $\begin{array}{c}1692 \pm 262.1 \\
(325-2775)\end{array}$ & $\begin{array}{l}821 \pm 94.6 \\
(446-1285)\end{array}$ & $\begin{array}{c}1504 \pm 301.3 \\
(256-3062)\end{array}$ & $\begin{array}{c}1 \pm 0.3 \\
(0-3)\end{array}$ & $\begin{array}{c}0 \pm 0 \\
(\mathrm{ND}-0.008)\end{array}$ & $\begin{array}{l}1 \pm 0.2 \\
(0.5-2)\end{array}$ \\
\hline NMWI-1 & 10 & $\begin{array}{c}433 \pm 8 \\
(405-490)\end{array}$ & $\begin{array}{l}980 \pm 62.2 \\
(712-1430)\end{array}$ & $\begin{array}{c}656 \pm 51 \\
(365-859)\end{array}$ & $\begin{array}{c}707 \pm 100 \\
(253-1306)\end{array}$ & $\begin{array}{c}1 \pm 0.2 \\
(0-3)\end{array}$ & $\begin{array}{c}0 \pm 0 \\
(\mathrm{ND}-0.02)\end{array}$ & $\begin{array}{c}2 \pm 0.3 \\
(1-3)\end{array}$ \\
\hline NMWI-2 & 23 & $\begin{array}{l}486 \pm 8.9 \\
(414-560)\end{array}$ & $\begin{array}{l}1384 \pm 74.6 \\
(868-2154)\end{array}$ & $\begin{array}{l}775 \pm 65.3 \\
(214-1340)\end{array}$ & $\begin{array}{l}621 \pm 55.5 \\
(108-1118)\end{array}$ & $\begin{array}{c}1 \pm 0.2 \\
(1-4)\end{array}$ & $\begin{array}{c}1 \pm 0.7 \\
(\mathrm{ND}-15)\end{array}$ & $\begin{array}{c}3 \pm 0.1 \\
(2-3)\end{array}$ \\
\hline NMWI-3 & 12 & $\begin{array}{l}519 \pm 8.3 \\
(468-578)\end{array}$ & $\begin{array}{l}1817 \pm 81.7 \\
(1400-2400)\end{array}$ & $\begin{array}{c}582 \pm 109.2 \\
(145-1474)\end{array}$ & $\begin{array}{c}1661 \pm 249.4 \\
(191-2893)\end{array}$ & $\begin{array}{c}1 \pm 0.6 \\
(0-8)\end{array}$ & $\begin{array}{c}0 \pm 0 \\
(\mathrm{ND}-0.005)\end{array}$ & $\begin{array}{c}1 \pm 0.1 \\
(1-2)\end{array}$ \\
\hline Summary & 384 & $\begin{array}{l}505 \pm 3.7 \\
(299-722)\end{array}$ & $\begin{array}{c}1752 \pm 38.1 \\
(324-6407)\end{array}$ & $\begin{array}{c}531 \pm 21.1 \\
(17-2918)\end{array}$ & $\begin{array}{c}1288 \pm 54.9 \\
(69-6796)\end{array}$ & $\begin{array}{c}1 \pm 0.1 \\
(0-19)\end{array}$ & $\begin{array}{c}0 \pm 0.1 \\
(\mathrm{ND}-25.427)\end{array}$ & $\begin{array}{l}2 \pm 0 \\
(0-3)\end{array}$ \\
\hline
\end{tabular}


Table 3A. (Continued) Site and regional mean values for biomarker and ancillary data for COMMON CARP collections made at all sites in U.S. waters from March 1994 through August 1997.

[N, number of fish; E2, 17ß Estradiol; 11KT, 11 ketotestosterone; VTG, vitellogenin; mm, millimeter; g, grams; pg, picograms; mg, milligram; mL, milliliter; $\mathrm{ND}$, nondetected]

\begin{tabular}{|c|c|c|c|c|c|c|c|c|}
\hline $\begin{array}{c}\text { Regional site } \\
\text { code } \\
\text { (fig. 1) }\end{array}$ & $\mathbf{N}$ & $\begin{array}{l}\text { Length } \\
(\mathrm{mm})\end{array}$ & $\begin{array}{l}\text { Weight } \\
\text { (g) }\end{array}$ & $\underset{(p g / m L}{E_{2}}$ & $\begin{array}{c}11 \mathrm{KT} \\
(\mathrm{pg} / \mathrm{mL})\end{array}$ & $\begin{array}{l}\text { Ratio of } \\
E_{2} \text { to KT }\end{array}$ & $\underset{(\mathrm{mg} / \mathrm{mL})}{\text { VTG }}$ & $\begin{array}{c}\text { Developmental } \\
\text { stage }\end{array}$ \\
\hline \multicolumn{9}{|c|}{ Male reproductive } \\
\hline NMIN-1 & 5 & $\begin{array}{l}583 \pm 4.9 \\
(572-599)\end{array}$ & $\begin{array}{l}2973 \pm 95.8 \\
(2675-3235)\end{array}$ & $\begin{array}{c}173 \pm 38.3 \\
(101-308)\end{array}$ & $\begin{array}{l}690 \pm 148.2 \\
(376-1092)\end{array}$ & $\begin{array}{l}0 \pm 0 \\
(0-0)\end{array}$ & $\begin{array}{c}0 \pm 0 \\
(\mathrm{ND}-0.001)\end{array}$ & $\begin{array}{l}3 \pm 0 \\
(3-3)\end{array}$ \\
\hline NMIN-3 & 19 & $\begin{array}{l}539 \pm 13.9 \\
(458-653)\end{array}$ & $\begin{array}{c}2343 \pm 158.5 \\
(1340-3722)\end{array}$ & $\begin{array}{c}278 \pm 21.4 \\
(108-441)\end{array}$ & $\begin{array}{l}573 \pm 77.5 \\
(151-1267)\end{array}$ & $\begin{array}{c}1 \pm 0.1 \\
(0-2)\end{array}$ & $\begin{array}{c}1 \pm 0.6 \\
(\mathrm{ND}-11.956)\end{array}$ & $\begin{array}{c}3 \pm 0.1 \\
(1-3)\end{array}$ \\
\hline Summary & 24 & $\begin{array}{l}548 \pm 11.6 \\
(458-653)\end{array}$ & $\begin{array}{c}2475 \pm 136.9 \\
(1340-3722)\end{array}$ & $\begin{array}{c}256 \pm 20.3 \\
(101-441)\end{array}$ & $\begin{array}{l}597 \pm 67.9 \\
(151-1267)\end{array}$ & $\begin{array}{c}1 \pm 0.1 \\
(0-2)\end{array}$ & $\begin{array}{c}1 \pm 0.5 \\
(\mathrm{ND}-11.956)\end{array}$ & $\begin{array}{c}3 \pm 0.1 \\
(1-3)\end{array}$ \\
\hline
\end{tabular}

\section{Female non-reproductive}

\begin{tabular}{|c|c|c|c|c|c|c|c|c|}
\hline NECT-1 & 9 & $\begin{array}{l}729 \pm 19.8 \\
(618-810)\end{array}$ & $\begin{array}{c}6613 \pm 581.2 \\
(2423-8286)\end{array}$ & $\begin{array}{c}3343 \pm 644 \\
(1646-7703)\end{array}$ & $\begin{array}{c}1568 \pm 191.3 \\
(769-2740)\end{array}$ & $\begin{array}{c}2 \pm 0.3 \\
(1-5)\end{array}$ & $\begin{array}{c}29 \pm 4.2 \\
(\mathrm{ND}-38.3)\end{array}$ & $\begin{array}{c}3 \pm 0.2 \\
(3-4)\end{array}$ \\
\hline NEDC-1 & 10 & $\begin{array}{l}591 \pm 22.8 \\
(496-692)\end{array}$ & $\begin{array}{l}3493 \pm 373.2 \\
(2032-5477)\end{array}$ & $\begin{array}{l}787 \pm 124 \\
(323-1765)\end{array}$ & $\begin{array}{l}552 \pm 129 \\
(66-1125)\end{array}$ & $\begin{array}{l}3 \pm 1.2 \\
(1-12)\end{array}$ & $\begin{array}{c}24 \pm 3 \\
(6.64-39.6)\end{array}$ & $\begin{array}{c}3 \pm 0.1 \\
(3-4)\end{array}$ \\
\hline NENY-1 & 7 & $\begin{array}{l}556 \pm 32 \\
(487-727)\end{array}$ & $\begin{array}{l}3023 \pm 591.5 \\
(1569-6200)\end{array}$ & $\begin{array}{c}2694 \pm 659.6 \\
(1002-5780)\end{array}$ & $\begin{array}{l}336 \pm 101 \\
(113-920)\end{array}$ & $\begin{array}{l}11 \pm 3 \\
(4-22)\end{array}$ & $\begin{array}{c}20 \pm 2.5 \\
(8-29)\end{array}$ & $\begin{array}{l}3 \pm 0 \\
(3-3)\end{array}$ \\
\hline NENY-2 & 16 & $\begin{array}{l}561 \pm 9.3 \\
(486-614)\end{array}$ & $\begin{array}{c}2774 \pm 129 \\
(1813-3516)\end{array}$ & $\begin{array}{c}1746 \pm 342.1 \\
(562-4741)\end{array}$ & $\begin{array}{c}586 \pm 101.1 \\
(115-1508)\end{array}$ & $\begin{array}{l}5 \pm 1.7 \\
(1-22)\end{array}$ & $\begin{array}{c}14 \pm 1.4 \\
(6.4-26.6)\end{array}$ & $\begin{array}{l}3 \pm 0 \\
(3-3)\end{array}$ \\
\hline NENY-4 & 11 & $\begin{array}{c}635 \pm 35.3 \\
(513-920)\end{array}$ & $\begin{array}{l}4104 \pm 749.7 \\
(2175-10000)\end{array}$ & $\begin{array}{c}3329 \pm 388.8 \\
(435-4499)\end{array}$ & $\begin{array}{c}1102 \pm 146.4 \\
(304-1702)\end{array}$ & $\begin{array}{c}3 \pm 0.5 \\
(1-8)\end{array}$ & $\begin{array}{l}35 \pm 2.5 \\
(19-50.2)\end{array}$ & $\begin{array}{l}3 \pm 0 \\
(3-3)\end{array}$ \\
\hline NEOH-1 & 2 & $\begin{array}{l}623 \pm 87.5 \\
(535-710)\end{array}$ & $\begin{array}{l}3225 \pm 1425 \\
(1800-4650)\end{array}$ & $\begin{array}{c}424 \pm 145.5 \\
(278-569)\end{array}$ & $\begin{array}{l}137 \pm 13.5 \\
(123-150)\end{array}$ & $\begin{array}{c}3 \pm 0.8 \\
(2-4)\end{array}$ & $\begin{array}{c}3 \pm 1 \\
(2.18-4.23)\end{array}$ & $\begin{array}{c}3 \pm 0.5 \\
(2-3)\end{array}$ \\
\hline NEPA-1 & 6 & $\begin{array}{c}519 \pm 35.9 \\
(429-660)\end{array}$ & $\begin{array}{c}1975 \pm 463.3 \\
(1000-3900)\end{array}$ & $\begin{array}{l}1166 \pm 452 \\
(259-2768)\end{array}$ & $\begin{array}{c}467 \pm 221.7 \\
(88-1504)\end{array}$ & $\begin{array}{c}3 \pm 0.7 \\
(1-6)\end{array}$ & $\begin{array}{c}2 \pm 0.6 \\
(0.314-4.671)\end{array}$ & $\begin{array}{l}3 \pm 0 \\
(3-3)\end{array}$ \\
\hline NEWV-1 & 14 & $\begin{array}{l}629 \pm 14.1 \\
(518-726)\end{array}$ & $\begin{array}{l}3740 \pm 220 \\
(1834-5335)\end{array}$ & $\begin{array}{c}1159 \pm 125.7 \\
(315-1760)\end{array}$ & $\begin{array}{c}278 \pm 53.1 \\
(13-700)\end{array}$ & $\begin{array}{l}9 \pm 2.5 \\
(1-28)\end{array}$ & $\begin{array}{c}14 \pm 1.5 \\
(\mathrm{ND}-20.4)\end{array}$ & $\begin{array}{c}3 \pm 0.1 \\
(3-4)\end{array}$ \\
\hline NEWV-2 & 11 & $\begin{array}{l}406 \pm 8.7 \\
(362-442)\end{array}$ & $\begin{array}{c}875 \pm 54.9 \\
(584.4-1204.8)\end{array}$ & $\begin{array}{l}716 \pm 92.8 \\
(309-1231)\end{array}$ & $\begin{array}{c}262 \pm 33.3 \\
(103-481)\end{array}$ & $\begin{array}{c}3 \pm 0.4 \\
(1-6)\end{array}$ & $\begin{array}{c}1 \pm 0.3 \\
(\mathrm{ND}-2.887)\end{array}$ & $\begin{array}{l}3 \pm 0 \\
(3-3)\end{array}$ \\
\hline Summary & 86 & $\begin{array}{c}581 \pm 11.8 \\
(362-920)\end{array}$ & $\begin{array}{c}3319 \pm 211.2 \\
(584.4-10000)\end{array}$ & $\begin{array}{c}1783 \pm 162.1 \\
(259-7703)\end{array}$ & $\begin{array}{l}620 \pm 60.1 \\
(13-2740)\end{array}$ & $\begin{array}{l}5 \pm 0.6 \\
(1-28)\end{array}$ & $\begin{array}{c}17 \pm 1.4 \\
(\mathrm{ND}-50.2)\end{array}$ & $\begin{array}{l}3 \pm 0 \\
(2-4)\end{array}$ \\
\hline \multicolumn{9}{|c|}{ Female reproductive } \\
\hline NENY-1 & 3 & $\begin{array}{l}575 \pm 23.9 \\
(529-610)\end{array}$ & $\begin{array}{c}2990 \pm 482.1 \\
(2070-3700)\end{array}$ & $\begin{array}{c}683 \pm 147.3 \\
(433-943)\end{array}$ & $\begin{array}{l}799 \pm 331.1 \\
(167-1286)\end{array}$ & $\begin{array}{c}1 \pm 0.6 \\
(1-3)\end{array}$ & $\begin{array}{c}57 \pm 15.5 \\
(40.36-87.793)\end{array}$ & $\begin{array}{l}3 \pm 0 \\
(3-3)\end{array}$ \\
\hline NENY-2 & 12 & $\begin{array}{l}585 \pm 18.1 \\
(455-689)\end{array}$ & $\begin{array}{l}3392 \pm 279.5 \\
(2100-5300)\end{array}$ & $\begin{array}{c}2753 \pm 297.6 \\
(1159-4142)\end{array}$ & $\begin{array}{c}3333 \pm 307.1 \\
(1456-5066)\end{array}$ & $\begin{array}{c}1 \pm 0.1 \\
(0-2)\end{array}$ & $\begin{array}{c}37 \pm 5.3 \\
(13.348-65.612)\end{array}$ & $\begin{array}{l}3 \pm 0 \\
(3-3)\end{array}$ \\
\hline NENY-4 & 13 & $\begin{array}{c}658 \pm 14 \\
(576-735)\end{array}$ & $\begin{array}{l}4715 \pm 346 \\
(2800-6300)\end{array}$ & $\begin{array}{c}1722 \pm 234.2 \\
(467-3662)\end{array}$ & $\begin{array}{l}1004 \pm 195 \\
(199-2554)\end{array}$ & $\begin{array}{l}3 \pm 0.8 \\
(1-12)\end{array}$ & $\begin{array}{c}44 \pm 6.1 \\
(\mathrm{ND}-89.456)\end{array}$ & $\begin{array}{l}3 \pm 0 \\
(3-3)\end{array}$ \\
\hline Summary & 28 & $\begin{array}{l}618 \pm 12.5 \\
(455-735)\end{array}$ & $\begin{array}{c}3963 \pm 242.6 \\
(2070-6300)\end{array}$ & $\begin{array}{c}2053 \pm 209.9 \\
(433-4142)\end{array}$ & $\begin{array}{l}1980 \pm 276 \\
(167-5066)\end{array}$ & $\begin{array}{l}2 \pm 0.4 \\
(0-12)\end{array}$ & $\begin{array}{c}42 \pm 4 \\
(\mathrm{ND}-89.456)\end{array}$ & $\begin{array}{l}3 \pm 0 \\
(3-3)\end{array}$ \\
\hline
\end{tabular}


Table 3A. (Continued) Site and regional mean values for biomarker and ancillary data for COMMON CARP collections made at all sites in U.S. waters from March 1994 through August 1997.

[N, number of fish; E2, 17ß Estradiol; 11KT, 11 ketotestosterone; VTG, vitellogenin; mm, millimeter; g, grams; pg, picograms; mg, milligram; mL, milliliter;

ND, nondetected]

\begin{tabular}{|c|c|c|c|c|c|c|c|c|}
\hline $\begin{array}{c}\text { Regional site } \\
\text { code } \\
\text { (fig. 1) }\end{array}$ & $\mathbf{N}$ & $\begin{array}{l}\text { Length } \\
\text { (mm) }\end{array}$ & $\begin{array}{l}\text { Weight } \\
\text { (g) }\end{array}$ & $\underset{(p g / m L)}{E_{2}}$ & $\begin{array}{c}11 \mathrm{KT} \\
(\mathrm{pg} / \mathrm{mL})\end{array}$ & $\begin{array}{l}\text { Ratio of } \\
E_{2} \text { to KT }\end{array}$ & $\underset{(\mathrm{mg} / \mathrm{mL})}{\mathrm{VTG}}$ & $\begin{array}{c}\text { Developmental } \\
\text { stage }\end{array}$ \\
\hline \multicolumn{9}{|c|}{$\square$ Male non-reproductive } \\
\hline NECT-1 & 11 & $\begin{array}{c}569 \pm 37.9 \\
(339-690)\end{array}$ & $\begin{array}{c}3268 \pm 632.8 \\
(601-6356)\end{array}$ & $\begin{array}{l}744 \pm 102.1 \\
(114-1227)\end{array}$ & $\begin{array}{c}1349 \pm 175.7 \\
(442-2161)\end{array}$ & $\begin{array}{c}1 \pm 0.1 \\
(0-1)\end{array}$ & $\begin{array}{c}0 \pm 0 \\
(\mathrm{ND}-0.001)\end{array}$ & $\begin{array}{c}3 \pm 0.1 \\
(2-3)\end{array}$ \\
\hline NEDC-1 & 22 & $\begin{array}{c}508 \pm 10.3 \\
(401-615)\end{array}$ & $\begin{array}{c}2068 \pm 123.8 \\
(1073-3545)\end{array}$ & $\begin{array}{l}391 \pm 24.8 \\
(204-601)\end{array}$ & $\begin{array}{l}911 \pm 113.3 \\
(341-2038)\end{array}$ & $\begin{array}{c}1 \pm 0.1 \\
(0-1)\end{array}$ & $\begin{array}{c}0 \pm 0 \\
(\mathrm{ND}-0.001)\end{array}$ & $\begin{array}{c}1 \pm 0.1 \\
(1-3)\end{array}$ \\
\hline NENY-1 & 19 & $\begin{array}{l}557 \pm 14.1 \\
(475-674)\end{array}$ & $\begin{array}{c}2628 \pm 250.9 \\
(1356-5500)\end{array}$ & $\begin{array}{l}506 \pm 80.6 \\
(72-1183)\end{array}$ & $\begin{array}{l}857 \pm 91.8 \\
(254-1638)\end{array}$ & $\begin{array}{c}1 \pm 0.1 \\
(0-1)\end{array}$ & $\begin{array}{c}0 \pm 0 \\
(\mathrm{ND}-0.57)\end{array}$ & $\begin{array}{c}3 \pm 0.1 \\
(2-3)\end{array}$ \\
\hline NENY-2 & 24 & $\begin{array}{c}539 \pm 5 \\
(466-576)\end{array}$ & $\begin{array}{l}2212 \pm 66.7 \\
(1582-3037)\end{array}$ & $\begin{array}{c}190 \pm 29.6 \\
(30-509)\end{array}$ & $\begin{array}{c}837 \pm 113.8 \\
(144-2186)\end{array}$ & $\begin{array}{c}0 \pm 0.1 \\
(0-1)\end{array}$ & $\begin{array}{c}0 \pm 0 \\
(\mathrm{ND}-0.18)\end{array}$ & $\begin{array}{c}2 \pm 0.2 \\
(1-3)\end{array}$ \\
\hline NENY-4 & 18 & $\begin{array}{c}607 \pm 10.9 \\
(508-673)\end{array}$ & $\begin{array}{c}3461 \pm 201 \\
(1946-5352)\end{array}$ & $\begin{array}{c}216 \pm 45.5 \\
(29-857)\end{array}$ & $\begin{array}{c}1762 \pm 142.5 \\
(565-3012)\end{array}$ & $\begin{array}{l}0 \pm 0 \\
(0-0)\end{array}$ & $\begin{array}{c}0 \pm 0 \\
(\mathrm{ND}-0.04)\end{array}$ & $\begin{array}{l}3 \pm 0 \\
(3-3)\end{array}$ \\
\hline NEOH-1 & 3 & $\begin{array}{c}523 \pm 22.5 \\
(479-552)\end{array}$ & $\begin{array}{c}1624 \pm 246.8 \\
(1150-1981)\end{array}$ & $\begin{array}{l}458 \pm 47.4 \\
(401-552)\end{array}$ & $\begin{array}{c}1760 \pm 1620.8 \\
(97-5001)\end{array}$ & $\begin{array}{c}2 \pm 1.2 \\
(0-4)\end{array}$ & $\begin{array}{c}0 \pm 0 \\
(0.001-0.013)\end{array}$ & $\begin{array}{l}1 \pm 0 \\
(1-1)\end{array}$ \\
\hline NEPA-1 & 5 & $\begin{array}{c}472 \pm 39.3 \\
(373-594)\end{array}$ & $\begin{array}{c}1870 \pm 223.4 \\
(1500-2750)\end{array}$ & $\begin{array}{l}361 \pm 23.6 \\
(300-407)\end{array}$ & $\begin{array}{c}2356 \pm 564.7 \\
(1319-4090)\end{array}$ & $\begin{array}{l}0 \pm 0 \\
(0-0)\end{array}$ & $\begin{array}{c}0 \pm 0 \\
(\mathrm{ND}-0.027)\end{array}$ & $\begin{array}{c}2 \pm 0.2 \\
(2-3)\end{array}$ \\
\hline NEWV-1 & 22 & $\begin{array}{l}577 \pm 6.7 \\
(507-637)\end{array}$ & $\begin{array}{l}2802 \pm 111.5 \\
(1832-3764)\end{array}$ & $\begin{array}{c}260 \pm 39.2 \\
(50-872)\end{array}$ & $\begin{array}{l}421 \pm 62.9 \\
(75-1187)\end{array}$ & $\begin{array}{c}1 \pm 0.1 \\
(0-2)\end{array}$ & $\begin{array}{c}0 \pm 0 \\
(\mathrm{ND}-0.27)\end{array}$ & $\begin{array}{c}1 \pm 0.1 \\
(1-2)\end{array}$ \\
\hline NEWV-2 & 8 & $\begin{array}{l}389 \pm 16.9 \\
(337-464)\end{array}$ & $\begin{array}{c}753 \pm 97 \\
(462.8-1129.5)\end{array}$ & $\begin{array}{l}301 \pm 37.9 \\
(135-419)\end{array}$ & $\begin{array}{l}971 \pm 196.5 \\
(371-1764)\end{array}$ & $\begin{array}{c}0 \pm 0.1 \\
(0-1)\end{array}$ & $\begin{array}{c}0 \pm 0 \\
(\mathrm{ND}-0.001)\end{array}$ & $\begin{array}{c}3 \pm 0 \\
(2.5-2.5)\end{array}$ \\
\hline Summary & 132 & $\begin{array}{l}543 \pm 6.6 \\
(337-690)\end{array}$ & $\begin{array}{c}2490 \pm 94.8 \\
(462.8-6356)\end{array}$ & $\begin{array}{l}350 \pm 22.8 \\
(29-1227)\end{array}$ & $\begin{array}{c}1038 \pm 69.2 \\
(75-5001)\end{array}$ & $\begin{array}{l}1 \pm 0 \\
(0-4)\end{array}$ & $\begin{array}{c}0 \pm 0 \\
(\mathrm{ND}-0.57)\end{array}$ & $\begin{array}{c}2 \pm 0.1 \\
(1-3)\end{array}$ \\
\hline \multicolumn{9}{|c|}{ Male reproductive } \\
\hline NENY-1 & 17 & $\begin{array}{c}568 \pm 17.7 \\
(473-702)\end{array}$ & $\begin{array}{c}2856 \pm 306.5 \\
(1375-5150)\end{array}$ & $\begin{array}{c}827 \pm 72 \\
(427-1786)\end{array}$ & $\begin{array}{l}800 \pm 102.8 \\
(222-1811)\end{array}$ & $\begin{array}{c}1 \pm 0.2 \\
(0-4)\end{array}$ & $\begin{array}{c}0 \pm 0 \\
(\mathrm{ND}-0.001)\end{array}$ & $\begin{array}{c}3 \pm 0.2 \\
(1-3)\end{array}$ \\
\hline NENY-2 & 17 & $\begin{array}{l}555 \pm 8.3 \\
(445-595)\end{array}$ & $\begin{array}{l}2371 \pm 113.7 \\
(1200-3200)\end{array}$ & $\begin{array}{c}435 \pm 40.4 \\
(83-686)\end{array}$ & $\begin{array}{c}3480 \pm 388.8 \\
(1409-6821)\end{array}$ & $\begin{array}{l}0 \pm 0 \\
(0-0)\end{array}$ & $\begin{array}{c}0 \pm 0 \\
(\mathrm{ND}-0.001)\end{array}$ & $\begin{array}{c}3 \pm 0.1 \\
(2-3)\end{array}$ \\
\hline NENY-4 & 13 & $\begin{array}{c}648 \pm 20.9 \\
(496-763)\end{array}$ & $\begin{array}{c}4585 \pm 430.1 \\
(2500-7300)\end{array}$ & $\begin{array}{c}911 \pm 219.5 \\
(89-2565)\end{array}$ & $\begin{array}{c}1679 \pm 349.6 \\
(205-4871)\end{array}$ & $\begin{array}{c}1 \pm 0.4 \\
(0-6)\end{array}$ & $\begin{array}{c}0 \pm 0 \\
(\mathrm{ND}-0.202)\end{array}$ & $\begin{array}{c}2 \pm 0.2 \\
(1-3)\end{array}$ \\
\hline Summary & 47 & $\begin{array}{c}585 \pm 10.6 \\
(445-763)\end{array}$ & $\begin{array}{c}3158 \pm 211.2 \\
(1200-7300)\end{array}$ & $\begin{array}{l}709 \pm 72.7 \\
(83-2565)\end{array}$ & $\begin{array}{c}2012 \pm 241.5 \\
(205-6821)\end{array}$ & $\begin{array}{c}1 \pm 0.2 \\
(0-6)\end{array}$ & $\begin{array}{c}0 \pm 0 \\
(\mathrm{ND}-0.202)\end{array}$ & $\begin{array}{c}3 \pm 0.1 \\
(1-3)\end{array}$ \\
\hline
\end{tabular}

\section{Northwest Region}

\section{Female non-reproductive}

\begin{tabular}{lcccccccc}
\hline NWID-1 & 10 & $399 \pm 12.1$ & $892 \pm 80.8$ & $1094 \pm 160.3$ & $289 \pm 52.4$ & $5 \pm 1.1$ & $1 \pm 0.3$ & $1 \pm 0.2$ \\
& & $(355-464)$ & $(603-1289)$ & $(483-1990)$ & $(89-612)$ & $(2-12)$ & $(0.009-2.805)$ & $(0-2)$ \\
NWID-2 & 4 & $617 \pm 11.8$ & $3550 \pm 266.1$ & $1201 \pm 48.7$ & $535 \pm 155.1$ & $3 \pm 1.1$ & $4 \pm 1.3$ & $2 \pm 0$ \\
& & $(585-639)$ & $(2900-4200)$ & $(1115-1341)$ & $(186-844)$ & $(2-6)$ & $(1.437-7.368)$ & $(2-2)$ \\
NWOR-2 & \multirow{2}{*}{14} & $404 \pm 3.7$ & $843 \pm 33.1$ & $538 \pm 122.8$ & $386 \pm 78.5$ & $2 \pm 0.2$ & $9 \pm 2.9$ & $2 \pm 0.2$ \\
& & $(385-437)$ & $(681-1022)$ & $(67-1522)$ & $(22-1030)$ & $(1-3)$ & $(0.31-36.9)$ & $(1-3)$ \\
NWOR-3 & 10 & $573 \pm 19.5$ & $2724 \pm 307.3$ & $632 \pm 83$ & $431 \pm 96.1$ & $2 \pm 0.6$ & $0 \pm 0.1$ & $2 \pm 0.1$ \\
& & $(500-696)$ & $(1670-4500)$ & $(88-1031)$ & $(58-898)$ & $(1-6)$ & $(0-0.997)$ & $(2-3)$
\end{tabular}


Table 3A. (Continued) Site and regional mean values for biomarker and ancillary data for COMMON CARP collections made at all sites in U.S. waters from March 1994 through August 1997.

[N, number of fish; E2, 17ß Estradiol; 11KT, 11 ketotestosterone; VTG, vitellogenin; mm, millimeter; g, grams; pg, picograms; mg, milligram; mL, milliliter; $\mathrm{ND}$, nondetected]

\begin{tabular}{|c|c|c|c|c|c|c|c|c|}
\hline $\begin{array}{c}\text { Regional site } \\
\text { code } \\
\text { (fig. 1) }\end{array}$ & $\mathbf{N}$ & $\begin{array}{l}\text { Length } \\
(\mathrm{mm})\end{array}$ & $\begin{array}{l}\text { Weight } \\
\text { (g) }\end{array}$ & $\begin{array}{c}E_{2} \\
(p g / m L)\end{array}$ & $\begin{array}{c}11 \mathrm{KT} \\
(\mathrm{pg} / \mathrm{mL})\end{array}$ & $\begin{array}{l}\text { Ratio of } \\
E_{2} \text { to KT }\end{array}$ & $\underset{(\mathrm{mg} / \mathrm{mL})}{\text { VTG }}$ & $\begin{array}{c}\text { Developmental } \\
\text { stage }\end{array}$ \\
\hline NWOR-4 & 4 & $\begin{array}{c}677 \pm 53.4 \\
(562-771)\end{array}$ & $\begin{array}{c}5313 \pm 1252.1 \\
(2650-8000)\end{array}$ & $\begin{array}{l}578 \pm 164 \\
(183-899)\end{array}$ & $\begin{array}{c}196 \pm 58.9 \\
(82-349)\end{array}$ & $\begin{array}{c}3 \pm 0.9 \\
(1-5)\end{array}$ & $\begin{array}{c}0 \pm 0.2 \\
(0.067-0.994)\end{array}$ & $\begin{array}{l}2 \pm 0 \\
(2-2)\end{array}$ \\
\hline NWWA-3 & 11 & $\begin{array}{c}518 \pm 19.4 \\
(439-626)\end{array}$ & $\begin{array}{c}2041 \pm 257.7 \\
(1105-3750)\end{array}$ & $\begin{array}{c}1241 \pm 139.5 \\
(512-1833)\end{array}$ & $\begin{array}{c}270 \pm 44.9 \\
(53-613)\end{array}$ & $\begin{array}{l}6 \pm 0.8 \\
(1-10)\end{array}$ & $\begin{array}{c}2 \pm 0.2 \\
(1.355-2.986)\end{array}$ & $\begin{array}{l}2 \pm 0 \\
(2-2)\end{array}$ \\
\hline NWWA-4 & 10 & $\begin{array}{l}607 \pm 19.3 \\
(475-661)\end{array}$ & $\begin{array}{c}3178 \pm 303.8 \\
(1376-4550)\end{array}$ & $\begin{array}{l}512 \pm 86.4 \\
(102-855)\end{array}$ & $\begin{array}{c}262 \pm 51.6 \\
(51-548)\end{array}$ & $\begin{array}{c}3 \pm 0.6 \\
(1-7)\end{array}$ & $\begin{array}{c}1 \pm 0.2 \\
(0.126-2.327)\end{array}$ & $\begin{array}{c}2 \pm 0.1 \\
(1-2)\end{array}$ \\
\hline NWWA-5 & 10 & $\begin{array}{c}654 \pm 23.3 \\
(535-745)\end{array}$ & $\begin{array}{l}4219 \pm 551.1 \\
(2150-6738)\end{array}$ & $\begin{array}{c}958 \pm 108.3 \\
(518-1462)\end{array}$ & $\begin{array}{c}260 \pm 41.2 \\
(79-447)\end{array}$ & $\begin{array}{l}5 \pm 1 \\
(1-10)\end{array}$ & $\begin{array}{c}2 \pm 0.2 \\
(1.457-3.109)\end{array}$ & $\begin{array}{l}2 \pm 0 \\
(2-2)\end{array}$ \\
\hline NWWA-6 & 10 & $\begin{array}{l}520 \pm 18.7 \\
(428-615)\end{array}$ & $\begin{array}{c}1818 \pm 213.8 \\
(898-3150)\end{array}$ & $\begin{array}{l}1063 \pm 96.1 \\
(587-1418)\end{array}$ & $\begin{array}{l}361 \pm 67.5 \\
(100-770)\end{array}$ & $\begin{array}{l}4 \pm 1 \\
(2-12)\end{array}$ & $\begin{array}{c}2 \pm 0.2 \\
(0.537-2.885)\end{array}$ & $\begin{array}{c}2 \pm 0.1 \\
(1-2)\end{array}$ \\
\hline Summary & 83 & $\begin{array}{l}531 \pm 12 \\
(355-771)\end{array}$ & $\begin{array}{c}2386 \pm 178.5 \\
(603-8000)\end{array}$ & $\begin{array}{c}854 \pm 50.5 \\
(67-1990)\end{array}$ & $\begin{array}{l}329 \pm 24.6 \\
(22-1030)\end{array}$ & $\begin{array}{l}4 \pm 0.3 \\
(1-12)\end{array}$ & $\begin{array}{c}3 \pm 0.6 \\
(0.009-36.9)\end{array}$ & $\begin{array}{c}2 \pm 0.1 \\
(0-3)\end{array}$ \\
\hline \multicolumn{9}{|c|}{ Female reproductive } \\
\hline NWOR-1 & 15 & $\begin{array}{l}504 \pm 9.4 \\
(428-565)\end{array}$ & $\begin{array}{c}1959 \pm 129.4 \\
(1052-2983)\end{array}$ & $\begin{array}{c}823 \pm 127.9 \\
(249-2069)\end{array}$ & $\begin{array}{l}592 \pm 87.8 \\
(168-1124)\end{array}$ & $\begin{array}{c}2 \pm 0.4 \\
(0-5)\end{array}$ & $\begin{array}{c}41 \pm 8 \\
(2.66-118.839)\end{array}$ & $\begin{array}{c}3 \pm 0.2 \\
(1-3)\end{array}$ \\
\hline NWOR-2 & 15 & $\begin{array}{c}402 \pm 7 \\
(345-445)\end{array}$ & $\begin{array}{l}939 \pm 48.3 \\
(550-1216)\end{array}$ & $\begin{array}{c}443 \pm 89.8 \\
(17-1105)\end{array}$ & $\begin{array}{l}458 \pm 48 \\
(144-744)\end{array}$ & $\begin{array}{c}1 \pm 0.5 \\
(0-6)\end{array}$ & $\begin{array}{c}25 \pm 3.2 \\
(0.001-40.04)\end{array}$ & $\begin{array}{c}2 \pm 0.2 \\
(2-4)\end{array}$ \\
\hline NWWA-1 & 10 & $\begin{array}{l}505 \pm 9.7 \\
(453-542)\end{array}$ & $\begin{array}{l}1945 \pm 112.5 \\
(1454-2451)\end{array}$ & $\begin{array}{c}1289 \pm 115.9 \\
(464-1875)\end{array}$ & $\begin{array}{c}501 \pm 77.3 \\
(45-934)\end{array}$ & $\begin{array}{l}3 \pm 0.8 \\
(2-10)\end{array}$ & $\begin{array}{c}20 \pm 5.1 \\
(4.596-62.448)\end{array}$ & $\begin{array}{l}3 \pm 0 \\
(3-3)\end{array}$ \\
\hline NWWA-2 & 15 & $\begin{array}{c}650 \pm 14.3 \\
(530-710)\end{array}$ & $\begin{array}{l}4190 \pm 237 \\
(2192-5320)\end{array}$ & $\begin{array}{l}1517 \pm 153.7 \\
(1005-3542)\end{array}$ & $\begin{array}{c}741 \pm 68 \\
(476-1507)\end{array}$ & $\begin{array}{c}2 \pm 0.1 \\
(2-3)\end{array}$ & $\begin{array}{c}44 \pm 6.9 \\
(16.589-86.521)\end{array}$ & $\begin{array}{c}3 \pm 0.1 \\
(3-4)\end{array}$ \\
\hline Summary & 55 & $\begin{array}{c}516 \pm 13.6 \\
(345-710)\end{array}$ & $\begin{array}{c}2287 \pm 184.7 \\
(550-5320)\end{array}$ & $\begin{array}{l}993 \pm 84.6 \\
(17-3542)\end{array}$ & $\begin{array}{c}580 \pm 38 \\
(45-1507)\end{array}$ & $\begin{array}{l}2 \pm 0.2 \\
(0-10)\end{array}$ & $\begin{array}{c}34 \pm 3.4 \\
(0.001-118.839)\end{array}$ & $\begin{array}{c}3 \pm 0.1 \\
(1-4)\end{array}$ \\
\hline \multicolumn{9}{|c|}{$\square$ Male non-reproductive } \\
\hline NWID-1 & 10 & $\begin{array}{l}364 \pm 8.7 \\
(321-406)\end{array}$ & $\begin{array}{l}683 \pm 54.1 \\
(397-900)\end{array}$ & $\begin{array}{l}304 \pm 56.2 \\
(135-575)\end{array}$ & $\begin{array}{c}777 \pm 176.9 \\
(128-1503)\end{array}$ & $\begin{array}{c}1 \pm 0.2 \\
(0-2)\end{array}$ & $\begin{array}{l}0 \pm 0 \\
(0-0)\end{array}$ & $\begin{array}{c}2 \pm 0.2 \\
(2-3)\end{array}$ \\
\hline NWID-2 & 9 & $\begin{array}{c}540 \pm 18.2 \\
(440-601)\end{array}$ & $\begin{array}{c}2300 \pm 207.5 \\
(1300-3300)\end{array}$ & $\begin{array}{l}452 \pm 75.1 \\
(171-792)\end{array}$ & $\begin{array}{l}778 \pm 158.9 \\
(260-1638)\end{array}$ & $\begin{array}{c}1 \pm 0.3 \\
(0-3)\end{array}$ & $\begin{array}{l}0 \pm 0 \\
(0-0)\end{array}$ & $\begin{array}{c}2 \pm 0.2 \\
(2-3)\end{array}$ \\
\hline NWOR-2 & 15 & $\begin{array}{l}385 \pm 8.1 \\
(339-482)\end{array}$ & $\begin{array}{l}742 \pm 57.4 \\
(454-1476)\end{array}$ & $\begin{array}{l}169 \pm 53 \\
(23-875)\end{array}$ & $\begin{array}{c}730 \pm 107.3 \\
(84-1738)\end{array}$ & $\begin{array}{c}0 \pm 0.1 \\
(0-2)\end{array}$ & $\begin{array}{c}0 \pm 0 \\
(\mathrm{ND}-0.68)\end{array}$ & $\begin{array}{c}2 \pm 0.1 \\
(2-3)\end{array}$ \\
\hline NWOR-3 & 10 & $\begin{array}{c}514 \pm 20.3 \\
(383-625)\end{array}$ & $\begin{array}{l}1961 \pm 221 \\
(819-3400)\end{array}$ & $\begin{array}{c}384 \pm 103.1 \\
(118-1117)\end{array}$ & $\begin{array}{c}440 \pm 103.6 \\
(126-1156)\end{array}$ & $\begin{array}{c}1 \pm 0.3 \\
(0-4)\end{array}$ & $\begin{array}{c}0 \pm 0 \\
(0-0.035)\end{array}$ & $\begin{array}{l}3 \pm 0 \\
(3-3)\end{array}$ \\
\hline NWOR-4 & 10 & $\begin{array}{l}563 \pm 21.3 \\
(465-677)\end{array}$ & $\begin{array}{c}2586 \pm 287.6 \\
(1351-3800)\end{array}$ & $\begin{array}{c}374 \pm 84.2 \\
(63-882)\end{array}$ & $\begin{array}{l}316 \pm 36.7 \\
(147-556)\end{array}$ & $\begin{array}{c}1 \pm 0.3 \\
(0-3)\end{array}$ & $\begin{array}{c}0 \pm 0 \\
(0-0.01)\end{array}$ & $\begin{array}{l}3 \pm 0 \\
(3-3)\end{array}$ \\
\hline NWWA-3 & 9 & $\begin{array}{c}501 \pm 12.2 \\
(461-581)\end{array}$ & $\begin{array}{c}1473 \pm 104.4 \\
(1200-2200)\end{array}$ & $\begin{array}{c}316 \pm 70.8 \\
(109-798)\end{array}$ & $\begin{array}{c}663 \pm 103 \\
(195-1139)\end{array}$ & $\begin{array}{c}1 \pm 0.1 \\
(0-1)\end{array}$ & $\begin{array}{l}0 \pm 0 \\
(0-0)\end{array}$ & $\begin{array}{c}2 \pm 0.2 \\
(2-3)\end{array}$ \\
\hline NWWA-4 & 11 & $\begin{array}{l}523 \pm 25.4 \\
(416-667)\end{array}$ & $\begin{array}{c}1853 \pm 224.2 \\
(917-2950)\end{array}$ & $\begin{array}{c}237 \pm 34.1 \\
(102-432)\end{array}$ & $\begin{array}{c}423 \pm 64.1 \\
(103-703)\end{array}$ & $\begin{array}{c}1 \pm 0.2 \\
(0-2)\end{array}$ & $\begin{array}{c}0 \pm 0 \\
(0-0.037)\end{array}$ & $\begin{array}{c}2 \pm 0.2 \\
(2-3)\end{array}$ \\
\hline
\end{tabular}


Table 3A. (Continued) Site and regional mean values for biomarker and ancillary data for COMMON CARP collections made at all sites in U.S. waters from March 1994 through August 1997.

[N, number of fish; E2, 17ß Estradiol; 11KT, 11 ketotestosterone; VTG, vitellogenin; mm, millimeter; g, grams; pg, picograms; mg, milligram; mL, milliliter; ND, nondetected]

\begin{tabular}{|c|c|c|c|c|c|c|c|c|}
\hline $\begin{array}{c}\text { Regional site } \\
\text { code } \\
\text { (fig. 1) }\end{array}$ & $\mathbf{N}$ & $\begin{array}{l}\text { Length } \\
(\mathrm{mm})\end{array}$ & $\begin{array}{l}\text { Weight } \\
\text { (g) }\end{array}$ & $\underset{(p g / m L)}{E_{2}}$ & $\begin{array}{c}11 \mathrm{KT} \\
(\mathrm{pg} / \mathrm{mL})\end{array}$ & $\begin{array}{l}\text { Ratio of } \\
E_{2} \text { to KT }\end{array}$ & $\underset{(\mathrm{mg} / \mathrm{mL})}{\mathrm{VTG}}$ & $\begin{array}{c}\text { Developmental } \\
\text { stage }\end{array}$ \\
\hline NWWA-5 & 11 & $\begin{array}{l}595 \pm 11.6 \\
(560-667)\end{array}$ & $\begin{array}{c}2891 \pm 174.6 \\
(2200-4000)\end{array}$ & $\begin{array}{c}179 \pm 32.7 \\
(72-463)\end{array}$ & $\begin{array}{c}582 \pm 125.4 \\
(185-1648)\end{array}$ & $\begin{array}{c}0 \pm 0.1 \\
(0-1)\end{array}$ & $\begin{array}{c}0 \pm 0 \\
(0-0.009)\end{array}$ & $\begin{array}{c}2 \pm 0.2 \\
(2-3)\end{array}$ \\
\hline NWWA-6 & 10 & $\begin{array}{c}489 \pm 13.6 \\
(444-590)\end{array}$ & $\begin{array}{c}1437 \pm 139.8 \\
(1038-2500)\end{array}$ & $\begin{array}{c}323 \pm 77.2 \\
(76-783)\end{array}$ & $\begin{array}{l}651 \pm 104 \\
(123-1124)\end{array}$ & $\begin{array}{c}1 \pm 0.2 \\
(0-2)\end{array}$ & $\begin{array}{l}0 \pm 0 \\
(0-0)\end{array}$ & $\begin{array}{c}3 \pm 0.1 \\
(2-3)\end{array}$ \\
\hline Summary & 95 & $\begin{array}{l}492 \pm 9.3 \\
(321-677)\end{array}$ & $\begin{array}{c}1726 \pm 94.7 \\
(397-4000)\end{array}$ & $\begin{array}{c}293 \pm 23.2 \\
(23-1117)\end{array}$ & $\begin{array}{c}598 \pm 40.1 \\
(84-1738)\end{array}$ & $\begin{array}{c}1 \pm 0.1 \\
(0-4)\end{array}$ & $\begin{array}{c}0 \pm 0 \\
(0-0.68)\end{array}$ & $\begin{array}{c}3 \pm 0.1 \\
(2-3)\end{array}$ \\
\hline \multicolumn{9}{|c|}{ Male reproductive } \\
\hline NWOR-1 & 15 & $\begin{array}{l}461 \pm 9.2 \\
(379-521)\end{array}$ & $\begin{array}{c}1342 \pm 75.9 \\
(690-1996)\end{array}$ & $\begin{array}{c}561 \pm 49.8 \\
(169-818)\end{array}$ & $\begin{array}{c}1473 \pm 273.9 \\
(509-4310)\end{array}$ & $\begin{array}{c}1 \pm 0.1 \\
(0-1)\end{array}$ & $\begin{array}{c}0 \pm 0 \\
(\mathrm{ND}-0.001)\end{array}$ & $\begin{array}{c}3 \pm 0.1 \\
(2-3)\end{array}$ \\
\hline NWOR-2 & 15 & $\begin{array}{l}369 \pm 7.1 \\
(327-446)\end{array}$ & $\begin{array}{l}726 \pm 35.2 \\
(547-1096)\end{array}$ & $\begin{array}{c}263 \pm 13.9 \\
(121-341)\end{array}$ & $\begin{array}{l}509 \pm 59.3 \\
(149-821)\end{array}$ & $\begin{array}{c}1 \pm 0.1 \\
(0-2)\end{array}$ & $\begin{array}{c}0 \pm 0 \\
(\mathrm{ND}-0.09)\end{array}$ & $\begin{array}{c}3 \pm 0.2 \\
(1-3)\end{array}$ \\
\hline NWWA-1 & 10 & $\begin{array}{l}511 \pm 12.2 \\
(464-571)\end{array}$ & $\begin{array}{r}1713 \pm 152 \\
(1056-2688)\end{array}$ & $\begin{array}{l}515 \pm 101.2 \\
(201-1179)\end{array}$ & $\begin{array}{c}1136 \pm 139.1 \\
(549-2020)\end{array}$ & $\begin{array}{c}0 \pm 0.1 \\
(0-1)\end{array}$ & $\begin{array}{c}0 \pm 0 \\
(\mathrm{ND}-0.001)\end{array}$ & $\begin{array}{c}2 \pm 0.3 \\
(1-3)\end{array}$ \\
\hline NWWA-2 & 15 & $\begin{array}{l}624 \pm 7.5 \\
(575-683)\end{array}$ & $\begin{array}{c}3404 \pm 118.1 \\
(2750-4012)\end{array}$ & $\begin{array}{l}554 \pm 78.9 \\
(226-1129)\end{array}$ & $\begin{array}{c}1038 \pm 129.1 \\
(393-2181)\end{array}$ & $\begin{array}{l}1 \pm 0 \\
(0-1)\end{array}$ & $\begin{array}{c}0 \pm 0 \\
(\mathrm{ND}-0.001)\end{array}$ & $\begin{array}{l}3 \pm 0 \\
(3-3)\end{array}$ \\
\hline Summary & 55 & $\begin{array}{l}489 \pm 13.7 \\
(327-683)\end{array}$ & $\begin{array}{c}1804 \pm 148.6 \\
(547-4012)\end{array}$ & $\begin{array}{l}469 \pm 35.2 \\
(121-1179)\end{array}$ & $\begin{array}{c}1030 \pm 98.6 \\
(149-4310)\end{array}$ & $\begin{array}{l}1 \pm 0 \\
(0-2)\end{array}$ & $\begin{array}{c}0 \pm 0 \\
(\mathrm{ND}-0.09)\end{array}$ & $\begin{array}{c}3 \pm 0.1 \\
(1-3)\end{array}$ \\
\hline
\end{tabular}

\section{Southern Mid-Continent Region}

\section{Female non-reproductive}

\begin{tabular}{|c|c|c|c|c|c|c|c|c|}
\hline SMAR-1 & 7 & $\begin{array}{l}471 \pm 28 \\
(340-550)\end{array}$ & $\begin{array}{c}1582 \pm 236.7 \\
(523-2278)\end{array}$ & $\begin{array}{c}2021 \pm 554.3 \\
(821-5126)\end{array}$ & $\begin{array}{c}194 \pm 20.5 \\
(99-268)\end{array}$ & $\begin{array}{c}11 \pm 2.9 \\
(3-21)\end{array}$ & $\begin{array}{c}4 \pm 0.5 \\
(2.155-5.763)\end{array}$ & $\begin{array}{c}3 \pm 0.1 \\
(2-3)\end{array}$ \\
\hline SMAR-2 & 10 & $\begin{array}{c}516 \pm 12.6 \\
(465-600)\end{array}$ & $\begin{array}{c}2022 \pm 187 \\
(1358-3472)\end{array}$ & $\begin{array}{c}1465 \pm 214.9 \\
(151-2192)\end{array}$ & $\begin{array}{c}615 \pm 155 \\
(203-1956)\end{array}$ & $\begin{array}{l}3 \pm 0.9 \\
(0-11)\end{array}$ & $\begin{array}{c}4 \pm 0.5 \\
(0.421-5.804)\end{array}$ & $\begin{array}{c}3 \pm 0.2 \\
(1-3)\end{array}$ \\
\hline SMAR-3 & 7 & $\begin{array}{c}599 \pm 21.3 \\
(540-700)\end{array}$ & $\begin{array}{c}3241 \pm 337.6 \\
(2052-4690)\end{array}$ & $\begin{array}{c}1105 \pm 255.4 \\
(439-2265)\end{array}$ & $\begin{array}{c}565 \pm 233.7 \\
(172-1925)\end{array}$ & $\begin{array}{c}3 \pm 0.8 \\
(0-7)\end{array}$ & $\begin{array}{c}1 \pm 0.6 \\
(\mathrm{ND}-4.28)\end{array}$ & $\begin{array}{l}3 \pm 0 \\
(3-3)\end{array}$ \\
\hline SMAR-4 & 9 & $\begin{array}{c}525 \pm 12.2 \\
(473-570)\end{array}$ & $\begin{array}{c}2026 \pm 150.7 \\
(1480-2792)\end{array}$ & $\begin{array}{c}1755 \pm 445.3 \\
(147-4537)\end{array}$ & $\begin{array}{c}376 \pm 65.7 \\
(166-779)\end{array}$ & $\begin{array}{c}4 \pm 0.8 \\
(1-9)\end{array}$ & $\begin{array}{c}3 \pm 0.5 \\
(1.122-5.995)\end{array}$ & $\begin{array}{l}3 \pm 0 \\
(3-3)\end{array}$ \\
\hline SMIL-2 & 5 & $\begin{array}{c}640 \pm 36.9 \\
(510-736)\end{array}$ & $\begin{array}{c}3304 \pm 744.7 \\
(1700-5720)\end{array}$ & $\begin{array}{c}462 \pm 77.9 \\
(349-769)\end{array}$ & $\begin{array}{c}409 \pm 152.9 \\
(108-779)\end{array}$ & $\begin{array}{c}3 \pm 1.2 \\
(0-7)\end{array}$ & $\begin{array}{c}2 \pm 0.4 \\
(0.845-3.24)\end{array}$ & $\begin{array}{l}3 \pm 0 \\
(3-3)\end{array}$ \\
\hline SMLA-2 & 7 & $\begin{array}{c}577 \pm 47.3 \\
(350-720)\end{array}$ & $\begin{array}{c}3790 \pm 784.5 \\
(720-6602)\end{array}$ & $\begin{array}{l}597 \pm 68.9 \\
(288-907)\end{array}$ & $\begin{array}{c}290 \pm 76.2 \\
(131-716)\end{array}$ & $\begin{array}{c}2 \pm 0.3 \\
(1-4)\end{array}$ & $\begin{array}{c}2 \pm 0.4 \\
(0.567-3.244)\end{array}$ & $\begin{array}{c}2 \pm 0.4 \\
(1-3)\end{array}$ \\
\hline SMLA-3 & 8 & $\begin{array}{c}531 \pm 38.1 \\
(435-750)\end{array}$ & $\begin{array}{c}2129 \pm 469.1 \\
(1097-5000)\end{array}$ & $\begin{array}{c}1211 \pm 125.3 \\
(591-1782)\end{array}$ & $\begin{array}{l}335 \pm 24 \\
(260-429)\end{array}$ & $\begin{array}{c}4 \pm 0.5 \\
(1-7)\end{array}$ & $\begin{array}{c}2 \pm 0.3 \\
(0.245-2.897)\end{array}$ & $\begin{array}{c}3 \pm 0.1 \\
(2-3)\end{array}$ \\
\hline SMMO-2 & 10 & $\begin{array}{l}375 \pm 7.7 \\
(344-415)\end{array}$ & $\begin{array}{c}663 \pm 59.8 \\
(304-990)\end{array}$ & $\begin{array}{c}491 \pm 94.8 \\
(181-984)\end{array}$ & $\begin{array}{c}226 \pm 34.8 \\
(123-461)\end{array}$ & $\begin{array}{c}3 \pm 0.6 \\
(1-7)\end{array}$ & $\begin{array}{c}2 \pm 0.5 \\
(0.006-3.998)\end{array}$ & $\begin{array}{c}2 \pm 0.3 \\
(1-3)\end{array}$ \\
\hline SMMS-3 & 1 & $\begin{array}{c}495 \pm 0 \\
(495-495)\end{array}$ & $\begin{array}{c}1638 \pm 0 \\
(1638-1638)\end{array}$ & $\begin{array}{c}1149 \pm 0 \\
(1149-1149)\end{array}$ & $\begin{array}{c}305 \pm 0 \\
(305-305)\end{array}$ & $\begin{array}{l}4 \pm 0 \\
(4-4)\end{array}$ & $\begin{array}{c}0 \pm 0 \\
(0.14-0.14)\end{array}$ & $\begin{array}{l}3 \pm 0 \\
(3-3)\end{array}$ \\
\hline SMOK-1 & 11 & $\begin{array}{c}534 \pm 17.7 \\
(473-670)\end{array}$ & $\begin{array}{c}2003 \pm 282.4 \\
(1250-4350)\end{array}$ & $\begin{array}{c}1426 \pm 218.6 \\
(271-2489)\end{array}$ & $\begin{array}{l}419 \pm 63.7 \\
(196-943)\end{array}$ & $\begin{array}{c}4 \pm 0.6 \\
(0-6)\end{array}$ & $\begin{array}{c}1 \pm 0.4 \\
(0.001-3.717)\end{array}$ & $\begin{array}{c}2 \pm 0.4 \\
(0-3)\end{array}$ \\
\hline
\end{tabular}


Table 3A. (Continued) Site and regional mean values for biomarker and ancillary data for COMMON CARP collections made at all sites in U.S. waters from March 1994 through August 1997.

[N, number of fish; E2, 17ß Estradiol; 11KT, 11 ketotestosterone; VTG, vitellogenin; mm, millimeter; g, grams; pg, picograms; mg, milligram; mL, milliliter; $\mathrm{ND}$, nondetected]

\begin{tabular}{|c|c|c|c|c|c|c|c|c|}
\hline $\begin{array}{l}\text { Regional site } \\
\text { code } \\
\text { (fig. 1) }\end{array}$ & $\mathbf{N}$ & $\begin{array}{l}\text { Length } \\
(\mathrm{mm})\end{array}$ & $\begin{array}{l}\text { Weight } \\
\text { (g) }\end{array}$ & $\underset{(p g / m L)}{E_{2}}$ & $\begin{array}{c}11 \mathrm{KT} \\
(\mathrm{pg} / \mathrm{mL})\end{array}$ & $\begin{array}{l}\text { Ratio of } \\
E_{2} \text { to KT }\end{array}$ & $\begin{array}{c}\text { VTG } \\
(\mathrm{mg} / \mathrm{mL})\end{array}$ & $\begin{array}{c}\text { Developmental } \\
\text { stage }\end{array}$ \\
\hline SMOK-2 & 10 & $\begin{array}{l}490 \pm 16.6 \\
(420-580)\end{array}$ & $\begin{array}{l}1603 \pm 143.1 \\
(1000-2550)\end{array}$ & $\begin{array}{c}1453 \pm 151.8 \\
(741-2466)\end{array}$ & $\begin{array}{l}891 \pm 86.4 \\
(464-1337)\end{array}$ & $\begin{array}{c}2 \pm 0.4 \\
(1-5)\end{array}$ & $\begin{array}{c}1 \pm 0.3 \\
(0.044-2.613)\end{array}$ & $\begin{array}{c}3 \pm 0.3 \\
(0-3)\end{array}$ \\
\hline SMOK-3 & 10 & $\begin{array}{l}540 \pm 20.5 \\
(470-656)\end{array}$ & $\begin{array}{c}2150 \pm 249.4 \\
(1400-3750)\end{array}$ & $\begin{array}{l}1622 \pm 116.7 \\
(1221-2303)\end{array}$ & $\begin{array}{c}960 \pm 143.8 \\
(199-1746)\end{array}$ & $\begin{array}{c}2 \pm 0.6 \\
(1-7)\end{array}$ & $\begin{array}{c}2 \pm 0.2 \\
(0.712-3.109)\end{array}$ & $\begin{array}{l}3 \pm 0 \\
(3-3)\end{array}$ \\
\hline SMTN-2 & 9 & $\begin{array}{l}451 \pm 15.5 \\
(379-504)\end{array}$ & $\begin{array}{c}1454 \pm 165.2 \\
(799-2200)\end{array}$ & $\begin{array}{c}444 \pm 109.6 \\
(186-1008)\end{array}$ & $\begin{array}{l}226 \pm 36.6 \\
(118-448)\end{array}$ & $\begin{array}{c}2 \pm 0.8 \\
(0-7)\end{array}$ & $\begin{array}{c}2 \pm 0.7 \\
(0.01-4.859)\end{array}$ & $\begin{array}{c}2 \pm 0.3 \\
(0-3)\end{array}$ \\
\hline SMTN-4 & 2 & $\begin{array}{c}545 \pm 0 \\
(545-545)\end{array}$ & $\begin{array}{l}2184 \pm 216 \\
(1968-2400)\end{array}$ & $\begin{array}{l}550 \pm 4.5 \\
(545-554)\end{array}$ & $\begin{array}{l}94 \pm 25 \\
(69-119)\end{array}$ & $\begin{array}{c}5 \pm 0.7 \\
(5-6)\end{array}$ & $\begin{array}{c}3 \pm 0.4 \\
(2.304-3.113)\end{array}$ & $\begin{array}{l}3 \pm 0 \\
(3-3)\end{array}$ \\
\hline SMTN-5 & 5 & $\begin{array}{l}543 \pm 38.7 \\
(455-662)\end{array}$ & $\begin{array}{c}2420 \pm 469.5 \\
(1200-3600)\end{array}$ & $\begin{array}{l}1470 \pm 327 \\
(583-2582)\end{array}$ & $\begin{array}{l}712 \pm 157 \\
(370-1249)\end{array}$ & $\begin{array}{c}2 \pm 0.4 \\
(1-3)\end{array}$ & $\begin{array}{c}0 \pm 0.2 \\
(0.001-1.24)\end{array}$ & $\begin{array}{c}3 \pm 0.2 \\
(2-3)\end{array}$ \\
\hline SMTX-3 & 5 & $\begin{array}{l}534 \pm 17.7 \\
(472-575)\end{array}$ & $\begin{array}{c}2110 \pm 152.8 \\
(1589-2423)\end{array}$ & $\begin{array}{c}382 \pm 77.4 \\
(110-558)\end{array}$ & $\begin{array}{c}119 \pm 31.2 \\
(57-237)\end{array}$ & $\begin{array}{c}3 \pm 0.6 \\
(2-5)\end{array}$ & $\begin{array}{l}12 \pm 1.8 \\
(7-16.5)\end{array}$ & $\begin{array}{l}3 \pm 0 \\
(3-3)\end{array}$ \\
\hline SMTX-4 & 5 & $\begin{array}{c}626 \pm 23.1 \\
(559-690)\end{array}$ & $\begin{array}{c}4106 \pm 597.3 \\
(2690-5992)\end{array}$ & $\begin{array}{c}1708 \pm 367.3 \\
(947-2922)\end{array}$ & $\begin{array}{l}445 \pm 63 \\
(265-654)\end{array}$ & $\begin{array}{c}4 \pm 0.4 \\
(2-4)\end{array}$ & $\begin{array}{l}10 \pm 1.2 \\
(7-12.8)\end{array}$ & $\begin{array}{l}3 \pm 0 \\
(3-3)\end{array}$ \\
\hline SMTX-7 & 13 & $\begin{array}{l}635 \pm 15.4 \\
(535-730)\end{array}$ & $\begin{array}{c}3292 \pm 239.8 \\
(1800-4600)\end{array}$ & $\begin{array}{l}733 \pm 48.6 \\
(538-1080)\end{array}$ & $\begin{array}{l}246 \pm 86.1 \\
(32-1136)\end{array}$ & $\begin{array}{l}8 \pm 1.9 \\
(1-26)\end{array}$ & $\begin{array}{c}1 \pm 0.2 \\
(0.265-3.142)\end{array}$ & $\begin{array}{l}3 \pm 0 \\
(3-3)\end{array}$ \\
\hline Summary & 134 & $\begin{array}{c}531 \pm 8 \\
(340-750)\end{array}$ & $\begin{array}{c}2268 \pm 107.3 \\
(304-6602)\end{array}$ & $\begin{array}{c}1145 \pm 69.4 \\
(110-5126)\end{array}$ & $\begin{array}{l}441 \pm 32.5 \\
(32-1956)\end{array}$ & $\begin{array}{l}4 \pm 0.3 \\
(0-26)\end{array}$ & $\begin{array}{c}3 \pm 0.3 \\
(\mathrm{ND}-16.5)\end{array}$ & $\begin{array}{c}3 \pm 0.1 \\
(0-3)\end{array}$ \\
\hline \multicolumn{9}{|c|}{ Female reproductive } \\
\hline SMLA-1 & 5 & $\begin{array}{l}479 \pm 29 \\
(404-554)\end{array}$ & $\begin{array}{c}1588 \pm 286.5 \\
(868-2380)\end{array}$ & $\begin{array}{l}977 \pm 317.1 \\
(166-2122)\end{array}$ & $\begin{array}{l}441 \pm 195.7 \\
(154-1209)\end{array}$ & $\begin{array}{c}3 \pm 0.8 \\
(1-5)\end{array}$ & $\begin{array}{c}3 \pm 1.1 \\
(0.202-5.641)\end{array}$ & $\begin{array}{l}3 \pm 0 \\
(3-3)\end{array}$ \\
\hline SMMS-1 & 8 & $\begin{array}{c}478 \pm 21.6 \\
(413-558)\end{array}$ & $\begin{array}{c}1411 \pm 189.2 \\
(907-2432)\end{array}$ & $\begin{array}{c}1125 \pm 207.7 \\
(23-1634)\end{array}$ & $\begin{array}{l}299 \pm 41.8 \\
(165-479)\end{array}$ & $\begin{array}{l}4 \pm 1 \\
(0-9)\end{array}$ & $\begin{array}{c}0 \pm 0 \\
(\mathrm{ND}-0.228)\end{array}$ & $\begin{array}{c}3 \pm 0.3 \\
(1-3)\end{array}$ \\
\hline SMMS-2 & 10 & $\begin{array}{l}445 \pm 19.5 \\
(356-528)\end{array}$ & $\begin{array}{c}1157 \pm 160.3 \\
(613-2316)\end{array}$ & $\begin{array}{c}1676 \pm 215.9 \\
(236-2318)\end{array}$ & $\begin{array}{c}539 \pm 108.2 \\
(88-1114)\end{array}$ & $\begin{array}{l}5 \pm 2.2 \\
(1-25)\end{array}$ & $\begin{array}{c}1 \pm 0.3 \\
(0.127-2.238)\end{array}$ & $\begin{array}{c}3 \pm 0.2 \\
(1-3)\end{array}$ \\
\hline SMMS-3 & 7 & $\begin{array}{l}392 \pm 16 \\
(332-456)\end{array}$ & $\begin{array}{l}843 \pm 90.7 \\
(479-1175)\end{array}$ & $\begin{array}{c}1534 \pm 269.1 \\
(338-2317)\end{array}$ & $\begin{array}{c}260 \pm 23.2 \\
(169-339)\end{array}$ & $\begin{array}{l}6 \pm 1.3 \\
(2-12)\end{array}$ & $\begin{array}{c}1 \pm 0.4 \\
(0.006-2.877)\end{array}$ & $\begin{array}{l}3 \pm 0 \\
(3-3)\end{array}$ \\
\hline SMMS-4 & 5 & $\begin{array}{l}609 \pm 42.2 \\
(497-742)\end{array}$ & $\begin{array}{c}3605 \pm 699.2 \\
(2071-6200)\end{array}$ & $\begin{array}{c}834 \pm 197.1 \\
(356-1391)\end{array}$ & $\begin{array}{l}635 \pm 155.5 \\
(427-1246)\end{array}$ & $\begin{array}{c}1 \pm 0.5 \\
(1-3)\end{array}$ & $\begin{array}{c}0 \pm 0.3 \\
(\mathrm{ND}-1.473)\end{array}$ & $\begin{array}{c}3 \pm 0.2 \\
(2-3)\end{array}$ \\
\hline SMTX-2 & 16 & $\begin{array}{l}499 \pm 18.6 \\
(420-710)\end{array}$ & $\begin{array}{c}1681 \pm 228.4 \\
(960-4521)\end{array}$ & $\begin{array}{c}1158 \pm 164.4 \\
(119-2397)\end{array}$ & $\begin{array}{l}477 \pm 79.7 \\
(112-1291)\end{array}$ & $\begin{array}{c}3 \pm 0.5 \\
(1-9)\end{array}$ & $\begin{array}{c}4 \pm 2.9 \\
(\mathrm{ND}-46.029)\end{array}$ & $\begin{array}{c}2 \pm 0.1 \\
(2-3)\end{array}$ \\
\hline Summary & 51 & $\begin{array}{l}479 \pm 12 \\
(332-742)\end{array}$ & $\begin{array}{c}1599 \pm 147.8 \\
(479-6200)\end{array}$ & $\begin{array}{c}1256 \pm 94.6 \\
(23-2397)\end{array}$ & $\begin{array}{l}443 \pm 42.7 \\
(88-1291)\end{array}$ & $\begin{array}{l}4 \pm 0.5 \\
(0-25)\end{array}$ & $\begin{array}{c}2 \pm 0.9 \\
(\mathrm{ND}-46.029)\end{array}$ & $\begin{array}{c}3 \pm 0.1 \\
(1-3)\end{array}$ \\
\hline \multicolumn{9}{|c|}{$\square$ Male non-reproductive } \\
\hline SMAR-1 & 9 & $\begin{array}{l}512 \pm 15.1 \\
(460-570)\end{array}$ & $\begin{array}{c}1809 \pm 146.5 \\
(1189-2505)\end{array}$ & $\begin{array}{l}626 \pm 172 \\
(61-1472)\end{array}$ & $\begin{array}{c}217 \pm 19.3 \\
(158-317)\end{array}$ & $\begin{array}{c}3 \pm 0.8 \\
(0-7)\end{array}$ & $\begin{array}{c}0 \pm 0 \\
(\mathrm{ND}-0.001)\end{array}$ & $\begin{array}{l}2 \pm 0 \\
(2-2)\end{array}$ \\
\hline SMAR-2 & 10 & $\begin{array}{l}465 \pm 13.5 \\
(391-519)\end{array}$ & $\begin{array}{c}1317 \pm 100.3 \\
(882-1758)\end{array}$ & $\begin{array}{c}410 \pm 97.9 \\
(83-946)\end{array}$ & $\begin{array}{l}990 \pm 190.2 \\
(109-1815)\end{array}$ & $\begin{array}{c}1 \pm 0.3 \\
(0-4)\end{array}$ & $\begin{array}{c}0 \pm 0 \\
(\mathrm{ND}-0.001)\end{array}$ & $\begin{array}{l}2 \pm 0 \\
(2-2)\end{array}$ \\
\hline
\end{tabular}


Table 3A. (Continued) Site and regional mean values for biomarker and ancillary data for COMMON CARP collections made at all sites in U.S. waters from March 1994 through August 1997.

[N, number of fish; E2, 17ß Estradiol; 11KT, 11 ketotestosterone; VTG, vitellogenin; mm, millimeter; g, grams; pg, picograms; mg, milligram; mL, milliliter; $\mathrm{ND}$, nondetected]

\begin{tabular}{|c|c|c|c|c|c|c|c|c|}
\hline $\begin{array}{c}\text { Regional site } \\
\text { code } \\
\text { (fig. 1) }\end{array}$ & $\mathbf{N}$ & $\begin{array}{l}\text { Length } \\
(\mathrm{mm})\end{array}$ & $\begin{array}{l}\text { Weight } \\
\text { (g) }\end{array}$ & $\underset{(p g / m L)}{E_{2}}$ & $\begin{array}{c}11 \mathrm{KT} \\
(\mathrm{pg} / \mathrm{mL})\end{array}$ & $\begin{array}{l}\text { Ratio of } \\
E_{2} \text { to KT }\end{array}$ & $\underset{(\mathrm{mg} / \mathrm{mL})}{\text { VTG }}$ & $\begin{array}{c}\text { Developmental } \\
\text { stage }\end{array}$ \\
\hline SMAR-3 & 10 & $\begin{array}{l}566 \pm 23.5 \\
(430-674)\end{array}$ & $\begin{array}{c}2773 \pm 382.2 \\
(1147-4996)\end{array}$ & $\begin{array}{c}535 \pm 55.3 \\
(358-945)\end{array}$ & $\begin{array}{l}1265 \pm 317 \\
(248-3226)\end{array}$ & $\begin{array}{c}1 \pm 0.2 \\
(0-2)\end{array}$ & $\begin{array}{c}0 \pm 0 \\
(\mathrm{ND}-0.001)\end{array}$ & $\begin{array}{c}2 \pm 0.2 \\
(1-3)\end{array}$ \\
\hline SMAR-4 & 10 & $\begin{array}{c}545 \pm 30.9 \\
(330-700)\end{array}$ & $\begin{array}{c}2016 \pm 302.9 \\
(643-4200)\end{array}$ & $\begin{array}{c}496 \pm 116.1 \\
(80-1228)\end{array}$ & $\begin{array}{c}3531 \pm 924.1 \\
(632-8492)\end{array}$ & $\begin{array}{c}0 \pm 0.1 \\
(0-1)\end{array}$ & $\begin{array}{c}0 \pm 0 \\
(0.001-0.014)\end{array}$ & $\begin{array}{l}1 \pm 0.1 \\
(1-1.5)\end{array}$ \\
\hline SMIL-2 & 6 & $\begin{array}{c}578 \pm 14.8 \\
(515-620)\end{array}$ & $\begin{array}{c}2550 \pm 282.3 \\
(1500-3600)\end{array}$ & $\begin{array}{c}300 \pm 47.7 \\
(170-469)\end{array}$ & $\begin{array}{c}2564 \pm 1379.1 \\
(271-7985)\end{array}$ & $\begin{array}{c}1 \pm 0.2 \\
(0-1)\end{array}$ & $\begin{array}{c}0 \pm 0 \\
(0.001-0.001)\end{array}$ & $\begin{array}{c}1 \pm 0.2 \\
(1-2)\end{array}$ \\
\hline SMLA-2 & 1 & $\begin{array}{c}600 \pm 0 \\
(600-600)\end{array}$ & $\begin{array}{c}3325 \pm 0 \\
(3325-3325)\end{array}$ & $\begin{array}{c}491 \pm 0 \\
(491-491)\end{array}$ & $\begin{array}{c}590 \pm 0 \\
(590-590)\end{array}$ & $\begin{array}{l}1 \pm 0 \\
(1-1)\end{array}$ & $\begin{array}{c}0 \pm 0 \\
(0.001-0.001)\end{array}$ & $\begin{array}{l}2 \pm 0 \\
(2-2)\end{array}$ \\
\hline SMLA-3 & 4 & $\begin{array}{c}554 \pm 45.3 \\
(473-651)\end{array}$ & $\begin{array}{c}2192 \pm 428.8 \\
(1410-2950)\end{array}$ & $\begin{array}{c}584 \pm 63.5 \\
(459-746)\end{array}$ & $\begin{array}{l}870 \pm 215 \\
(493-1296)\end{array}$ & $\begin{array}{c}1 \pm 0.2 \\
(0-1)\end{array}$ & $\begin{array}{c}0 \pm 0 \\
(0.001-0.001)\end{array}$ & $\begin{array}{c}2 \pm 0.3 \\
(2-3)\end{array}$ \\
\hline SMMO-2 & 10 & $\begin{array}{l}401 \pm 22.7 \\
(300-550)\end{array}$ & $\begin{array}{c}918 \pm 167.6 \\
(336-2009)\end{array}$ & $\begin{array}{l}369 \pm 31 \\
(259-527)\end{array}$ & $\begin{array}{c}1767 \pm 594.9 \\
(220-5991)\end{array}$ & $\begin{array}{c}0 \pm 0.1 \\
(0-1)\end{array}$ & $\begin{array}{c}0 \pm 0 \\
(0.001-0.001)\end{array}$ & $\begin{array}{c}2 \pm 0.2 \\
(1-2)\end{array}$ \\
\hline SMOK-1 & 9 & $\begin{array}{l}494 \pm 14.6 \\
(432-575)\end{array}$ & $\begin{array}{c}1578 \pm 134.7 \\
(1000-2500)\end{array}$ & $\begin{array}{l}339 \pm 21.4 \\
(246-461)\end{array}$ & $\begin{array}{l}865 \pm 176.1 \\
(359-2016)\end{array}$ & $\begin{array}{c}0 \pm 0.1 \\
(0-1)\end{array}$ & $\begin{array}{c}0 \pm 0 \\
(0.001-0.001)\end{array}$ & $\begin{array}{c}3 \pm 0.3 \\
(1-3)\end{array}$ \\
\hline SMOK-2 & 10 & $\begin{array}{l}435 \pm 10.1 \\
(356-470)\end{array}$ & $\begin{array}{l}1153 \pm 73.3 \\
(900-1700)\end{array}$ & $\begin{array}{c}1228 \pm 146.2 \\
(464-1957)\end{array}$ & $\begin{array}{c}1068 \pm 152.1 \\
(577-2348)\end{array}$ & $\begin{array}{c}1 \pm 0.2 \\
(0-3)\end{array}$ & $\begin{array}{c}0 \pm 0 \\
(0.001-0.001)\end{array}$ & $\begin{array}{c}2 \pm 0.1 \\
(2-3)\end{array}$ \\
\hline SMOK-3 & 9 & $\begin{array}{l}485 \pm 11.1 \\
(433-530)\end{array}$ & $\begin{array}{c}1500 \pm 117.2 \\
(1000-2175)\end{array}$ & $\begin{array}{l}775 \pm 98.5 \\
(300-1148)\end{array}$ & $\begin{array}{c}1536 \pm 191.7 \\
(652-2116)\end{array}$ & $\begin{array}{c}1 \pm 0.1 \\
(0-1)\end{array}$ & $\begin{array}{c}0 \pm 0 \\
(0.001-0.009)\end{array}$ & $\begin{array}{l}3 \pm 0 \\
(3-3)\end{array}$ \\
\hline SMTN-2 & 8 & $\begin{array}{l}445 \pm 14.5 \\
(370-498)\end{array}$ & $\begin{array}{c}1292 \pm 115.5 \\
(711-1778)\end{array}$ & $\begin{array}{l}413 \pm 85.7 \\
(205-828)\end{array}$ & $\begin{array}{l}499 \pm 159.3 \\
(167-1565)\end{array}$ & $\begin{array}{c}1 \pm 0.2 \\
(0-2)\end{array}$ & $\begin{array}{c}0 \pm 0 \\
(0.001-0.005)\end{array}$ & $\begin{array}{c}2 \pm 0.1 \\
(1-2)\end{array}$ \\
\hline SMTN-4 & 2 & $\begin{array}{c}538 \pm 32.5 \\
(505-570)\end{array}$ & $\begin{array}{c}2065 \pm 335.5 \\
(1729-2400)\end{array}$ & $\begin{array}{c}246 \pm 85.5 \\
(160-331)\end{array}$ & $\begin{array}{l}651 \pm 513.5 \\
(137-1164)\end{array}$ & $\begin{array}{c}1 \pm 1.1 \\
(0-2)\end{array}$ & $\begin{array}{c}0 \pm 0 \\
(0.001-0.025)\end{array}$ & $\begin{array}{l}2 \pm 0 \\
(2-2)\end{array}$ \\
\hline SMTN-5 & 10 & $\begin{array}{l}505 \pm 16.7 \\
(395-570)\end{array}$ & $\begin{array}{c}1745 \pm 177.4 \\
(850-2500)\end{array}$ & $\begin{array}{l}444 \pm 99.8 \\
(138-1276)\end{array}$ & $\begin{array}{c}1406 \pm 284.4 \\
(264-2783)\end{array}$ & $\begin{array}{c}1 \pm 0.5 \\
(0-5)\end{array}$ & $\begin{array}{c}0 \pm 0 \\
(0.001-0.001)\end{array}$ & $\begin{array}{l}2 \pm 0.1 \\
(1.5-3)\end{array}$ \\
\hline SMTX-3 & 2 & $\begin{array}{c}518 \pm 7 \\
(511-525)\end{array}$ & $\begin{array}{c}1749 \pm 33 \\
(1716-1782)\end{array}$ & $\begin{array}{l}78 \pm 0.5 \\
(77-78)\end{array}$ & $\begin{array}{l}85 \pm 11 \\
(74-96)\end{array}$ & $\begin{array}{c}1 \pm 0.1 \\
(1-1)\end{array}$ & $\begin{array}{c}0 \pm 0 \\
(\mathrm{ND}-0.001)\end{array}$ & $\begin{array}{l}1 \pm 0 \\
(1-1)\end{array}$ \\
\hline SMTX-4 & 4 & $\begin{array}{c}590 \pm 22.6 \\
(550-655)\end{array}$ & $\begin{array}{c}2969 \pm 107.4 \\
(2718-3155)\end{array}$ & $\begin{array}{l}303 \pm 60 \\
(128-388)\end{array}$ & $\begin{array}{c}616 \pm 47.8 \\
(521-732)\end{array}$ & $\begin{array}{c}0 \pm 0.1 \\
(0-1)\end{array}$ & $\begin{array}{c}0 \pm 0 \\
(\mathrm{ND}-0.001)\end{array}$ & $\begin{array}{c}2 \pm 0.3 \\
(1-2)\end{array}$ \\
\hline SMTX-5 & 1 & $\begin{array}{c}490 \pm 0 \\
(490-490)\end{array}$ & $\begin{array}{c}1550 \pm 0 \\
(1550-1550)\end{array}$ & $\begin{array}{c}636 \pm 0 \\
(636-636)\end{array}$ & $\begin{array}{c}1415 \pm 0 \\
(1415-1415)\end{array}$ & $\begin{array}{l}0 \pm 0 \\
(0-0)\end{array}$ & $\begin{array}{c}0 \pm 0 \\
(0.008-0.008)\end{array}$ & $\begin{array}{l}2 \pm 0 \\
(2-2)\end{array}$ \\
\hline SMTX-7 & 10 & $\begin{array}{c}585 \pm 15.4 \\
(521-670)\end{array}$ & $\begin{array}{l}2455 \pm 202 \\
(1700-3700)\end{array}$ & $\begin{array}{l}632 \pm 54.2 \\
(403-1037)\end{array}$ & $\begin{array}{c}1046 \pm 350.5 \\
(61-2983)\end{array}$ & $\begin{array}{l}4 \pm 1.5 \\
(0-13)\end{array}$ & $\begin{array}{c}0 \pm 0 \\
(0.001-0.093)\end{array}$ & $\begin{array}{c}2 \pm 0.1 \\
(2-3)\end{array}$ \\
\hline Summary & 125 & $\begin{array}{c}506 \pm 7 \\
(300-700)\end{array}$ & $\begin{array}{c}1816 \pm 74.6 \\
(336-4996)\end{array}$ & $\begin{array}{l}538 \pm 32.7 \\
(61-1957)\end{array}$ & $\begin{array}{c}1305 \pm 137.9 \\
(61-8492)\end{array}$ & $\begin{array}{l}1 \pm 0.2 \\
(0-13)\end{array}$ & $\begin{array}{c}0 \pm 0 \\
(\mathrm{ND}-0.093)\end{array}$ & $\begin{array}{c}2 \pm 0.1 \\
(1-3)\end{array}$ \\
\hline \multicolumn{9}{|c|}{ Male reproductive } \\
\hline SMLA-1 & 9 & $\begin{array}{l}518 \pm 11.6 \\
(445-555)\end{array}$ & $\begin{array}{l}1730 \pm 78.6 \\
(1298-2065)\end{array}$ & $\begin{array}{c}362 \pm 88.7 \\
(71-792)\end{array}$ & $\begin{array}{c}243 \pm 25.2 \\
(111-355)\end{array}$ & $\begin{array}{c}2 \pm 0.7 \\
(0-7)\end{array}$ & $\begin{array}{c}0 \pm 0 \\
(\mathrm{ND}-0.001)\end{array}$ & $\begin{array}{c}2 \pm 0.1 \\
(1-2)\end{array}$ \\
\hline SMMS-1 & 8 & $\begin{array}{c}447 \pm 20.6 \\
(368-548)\end{array}$ & $\begin{array}{c}1036 \pm 176.2 \\
(325-1829)\end{array}$ & $\begin{array}{c}286 \pm 77.4 \\
(78-786)\end{array}$ & $\begin{array}{l}438 \pm 71.1 \\
(201-738)\end{array}$ & $\begin{array}{c}1 \pm 0.2 \\
(0-1)\end{array}$ & $\begin{array}{c}0 \pm 0 \\
(\mathrm{ND}-0.178)\end{array}$ & $\begin{array}{l}1 \pm 0 \\
(1-1)\end{array}$ \\
\hline
\end{tabular}


Table 3A. (Continued) Site and regional mean values for biomarker and ancillary data for COMMON CARP collections made at all sites in U.S. waters from March 1994 through August 1997.

[N, number of fish; E2, 17ß Estradiol; 11KT, 11 ketotestosterone; VTG, vitellogenin; mm, millimeter; g, grams; pg, picograms; mg, milligram; mL, milliliter; $\mathrm{ND}$, nondetected]

\begin{tabular}{|c|c|c|c|c|c|c|c|c|}
\hline $\begin{array}{l}\text { Regional site } \\
\text { code } \\
\text { (fig. 1) }\end{array}$ & $\mathbf{N}$ & $\begin{array}{l}\text { Length } \\
(\mathrm{mm})\end{array}$ & $\begin{array}{l}\text { Weight } \\
\text { (g) }\end{array}$ & $\begin{array}{c}\mathrm{E}_{2} \\
(\mathrm{pg} / \mathrm{mL})\end{array}$ & $\begin{array}{c}11 \mathrm{KT} \\
(\mathrm{pg} / \mathrm{mL})\end{array}$ & $\begin{array}{l}\text { Ratio of } \\
E_{2} \text { to KT }\end{array}$ & $\begin{array}{c}\text { VTG } \\
(\mathrm{mg} / \mathrm{mL})\end{array}$ & $\begin{array}{c}\text { Developmental } \\
\text { stage }\end{array}$ \\
\hline SMMS-2 & 10 & $\begin{array}{l}444 \pm 16 \\
(383-546)\end{array}$ & $\begin{array}{c}1119 \pm 126.5 \\
(685-1977)\end{array}$ & $\begin{array}{c}197 \pm 69.3 \\
(51-757)\end{array}$ & $\begin{array}{l}688 \pm 73.9 \\
(372-1093)\end{array}$ & $\begin{array}{c}0 \pm 0.1 \\
(0-1)\end{array}$ & $\begin{array}{c}0 \pm 0 \\
(\mathrm{ND}-0.001)\end{array}$ & $\begin{array}{c}2 \pm 0.1 \\
(1-2)\end{array}$ \\
\hline SMMS-3 & 10 & $\begin{array}{c}401 \pm 10.6 \\
(362-460)\end{array}$ & $\begin{array}{c}824 \pm 75 \\
(649-1347)\end{array}$ & $\begin{array}{c}176 \pm 46.1 \\
(62-539)\end{array}$ & $\begin{array}{l}553 \pm 81.5 \\
(158-961)\end{array}$ & $\begin{array}{c}0 \pm 0.1 \\
(0-1)\end{array}$ & $\begin{array}{c}0 \pm 0 \\
(\mathrm{ND}-0.001)\end{array}$ & $\begin{array}{c}2 \pm 0.1 \\
(1-2)\end{array}$ \\
\hline SMMS-4 & 7 & $\begin{array}{c}527 \pm 41.8 \\
(297-657)\end{array}$ & $\begin{array}{c}2289 \pm 367.1 \\
(407-3693)\end{array}$ & $\begin{array}{l}526 \pm 96 \\
(120-924)\end{array}$ & $\begin{array}{l}465 \pm 64.3 \\
(199-721)\end{array}$ & $\begin{array}{c}1 \pm 0.4 \\
(0-3)\end{array}$ & $\begin{array}{c}0 \pm 0 \\
(\mathrm{ND}-0.001)\end{array}$ & $\begin{array}{c}2 \pm 0.1 \\
(1-2)\end{array}$ \\
\hline SMTX-2 & 9 & $\begin{array}{l}434 \pm 9.4 \\
(389-474)\end{array}$ & $\begin{array}{c}1066 \pm 71.3 \\
(661-1400)\end{array}$ & $\begin{array}{l}594 \pm 139.6 \\
(158-1442)\end{array}$ & $\begin{array}{c}1029 \pm 204.9 \\
(158-1945)\end{array}$ & $\begin{array}{c}1 \pm 0.5 \\
(0-5)\end{array}$ & $\begin{array}{c}0 \pm 0 \\
(\mathrm{ND}-0.001)\end{array}$ & $\begin{array}{c}2 \pm 0.1 \\
(2-3)\end{array}$ \\
\hline Summary & 53 & $\begin{array}{l}459 \pm 9.7 \\
(297-657)\end{array}$ & $\begin{array}{c}1309 \pm 91 \\
(325-3693)\end{array}$ & $\begin{array}{c}345 \pm 41 \\
(51-1442)\end{array}$ & $\begin{array}{l}578 \pm 53.3 \\
(111-1945)\end{array}$ & $\begin{array}{c}1 \pm 0.2 \\
(0-7)\end{array}$ & $\begin{array}{c}0 \pm 0 \\
(\mathrm{ND}-0.178)\end{array}$ & $\begin{array}{c}2 \pm 0.1 \\
(1-3)\end{array}$ \\
\hline
\end{tabular}

\section{Female non-reproductive}

\begin{tabular}{|c|c|c|c|c|c|c|c|c|}
\hline SWCA-1 & 22 & $\begin{array}{l}551 \pm 22 \\
(410-766)\end{array}$ & $\begin{array}{c}2413 \pm 265.6 \\
(943-5176)\end{array}$ & $\begin{array}{c}2669 \pm 334.1 \\
(614-6398)\end{array}$ & $\begin{array}{l}1225 \pm 193 \\
(215-2868)\end{array}$ & $\begin{array}{l}3 \pm 0.4 \\
(1-11)\end{array}$ & $\begin{array}{c}32 \pm 4.1 \\
(2.3-108.266)\end{array}$ & $\begin{array}{c}3 \pm 0.1 \\
(1-3)\end{array}$ \\
\hline SWCA-2 & 36 & $\begin{array}{l}514 \pm 18.1 \\
(323-742)\end{array}$ & $\begin{array}{c}2124 \pm 244.8 \\
(460-6356)\end{array}$ & $\begin{array}{c}1601 \pm 257.3 \\
(129-8333)\end{array}$ & $\begin{array}{c}1779 \pm 403.2 \\
(17-9066)\end{array}$ & $\begin{array}{c}2 \pm 0.3 \\
(0-9)\end{array}$ & $\begin{array}{c}23 \pm 2 \\
(1.04-42.6)\end{array}$ & $\begin{array}{c}3 \pm 0.1 \\
(1-4)\end{array}$ \\
\hline SWCA-3 & 36 & $\begin{array}{l}584 \pm 17.7 \\
(411-795)\end{array}$ & $\begin{array}{c}3255 \pm 296.2 \\
(913-7290)\end{array}$ & $\begin{array}{c}2328 \pm 376.7 \\
(135-11401)\end{array}$ & $\begin{array}{l}1278 \pm 232 \\
(121-5973)\end{array}$ & $\begin{array}{l}3 \pm 0.5 \\
(0-18)\end{array}$ & $\begin{array}{c}32 \pm 2.8 \\
(0.001-75.235)\end{array}$ & $\begin{array}{c}3 \pm 0.1 \\
(1-4)\end{array}$ \\
\hline SWUT-1 & 5 & $\begin{array}{l}611 \pm 41.4 \\
(495-700)\end{array}$ & $\begin{array}{c}3154 \pm 611.8 \\
(1540-4572)\end{array}$ & $\begin{array}{c}1256 \pm 240.4 \\
(791-2176)\end{array}$ & $\begin{array}{l}464 \pm 48.9 \\
(300-586)\end{array}$ & $\begin{array}{c}3 \pm 0.6 \\
(2-5)\end{array}$ & $\begin{array}{c}4 \pm 0.5 \\
(2.774-5.757)\end{array}$ & $\begin{array}{c}3 \pm 0.2 \\
(2-3)\end{array}$ \\
\hline SWUT-2 & 5 & $\begin{array}{l}415 \pm 22.5 \\
(366-492)\end{array}$ & $\begin{array}{c}1063 \pm 147.3 \\
(702-1572)\end{array}$ & $\begin{array}{l}784 \pm 152.2 \\
(366-1296)\end{array}$ & $\begin{array}{l}417 \pm 62.1 \\
(268-579)\end{array}$ & $\begin{array}{c}2 \pm 0.4 \\
(1-3)\end{array}$ & $\begin{array}{c}2 \pm 1.2 \\
(0.025-6.396)\end{array}$ & $\begin{array}{c}2 \pm 0.2 \\
(2-3)\end{array}$ \\
\hline SWUT-3 & 5 & $\begin{array}{l}723 \pm 29 \\
(625-785)\end{array}$ & $\begin{array}{c}5262 \pm 708.2 \\
(3397-7581)\end{array}$ & $\begin{array}{c}939 \pm 278.9 \\
(316-1816)\end{array}$ & $\begin{array}{c}564 \pm 111.2 \\
(311-838)\end{array}$ & $\begin{array}{c}2 \pm 0.4 \\
(1-3)\end{array}$ & $\begin{array}{c}1 \pm 0.3 \\
(0.581-2.198)\end{array}$ & $\begin{array}{l}3 \pm 0 \\
(3-3)\end{array}$ \\
\hline SWUT-4 & 6 & $\begin{array}{c}438 \pm 52.3 \\
(278-560)\end{array}$ & $\begin{array}{c}1427 \pm 440.5 \\
(288-2869)\end{array}$ & $\begin{array}{l}654 \pm 154 \\
(278-1207)\end{array}$ & $\begin{array}{c}231 \pm 48.9 \\
(34-364)\end{array}$ & $\begin{array}{l}4 \pm 1 \\
(1-8)\end{array}$ & $\begin{array}{c}1 \pm 0.5 \\
(0.042-3.172)\end{array}$ & $\begin{array}{c}2 \pm 0.4 \\
(1-3)\end{array}$ \\
\hline SWUT-5 & 4 & $\begin{array}{c}604 \pm 46.4 \\
(498-718)\end{array}$ & $\begin{array}{c}3082 \pm 861 \\
(1520-5500)\end{array}$ & $\begin{array}{c}759 \pm 166.8 \\
(575-1259)\end{array}$ & $\begin{array}{l}400 \pm 71 \\
(273-603)\end{array}$ & $\begin{array}{c}2 \pm 0.1 \\
(2-2)\end{array}$ & $\begin{array}{c}2 \pm 0.6 \\
(0.275-3.125)\end{array}$ & $\begin{array}{c}3 \pm 0.3 \\
(2-3)\end{array}$ \\
\hline Summary & 119 & $\begin{array}{c}549 \pm 10.8 \\
(278-795)\end{array}$ & $\begin{array}{c}2642 \pm 153.9 \\
(288-7581)\end{array}$ & $\begin{array}{c}1866 \pm 161.9 \\
(129-11401)\end{array}$ & $\begin{array}{c}1237 \pm 150.6 \\
(17-9066)\end{array}$ & $\begin{array}{c}2 \pm 0.2 \\
(0-18)\end{array}$ & $\begin{array}{c}23 \pm 1.7 \\
(0.001-108.266)\end{array}$ & $\begin{array}{c}3 \pm 0.1 \\
(1-4)\end{array}$ \\
\hline \multicolumn{9}{|c|}{ Female reproductive } \\
\hline SWAZ-1 & 10 & $\begin{array}{l}422 \pm 6.6 \\
(389-450)\end{array}$ & $\begin{array}{l}953 \pm 31.5 \\
(794-1118)\end{array}$ & $\begin{array}{l}450 \pm 70.3 \\
(187-904)\end{array}$ & $\begin{array}{l}446 \pm 59.1 \\
(171-857)\end{array}$ & $\begin{array}{c}1 \pm 0.1 \\
(1-2)\end{array}$ & $\begin{array}{c}2 \pm 0.5 \\
(0.317-5.816)\end{array}$ & $\begin{array}{c}3 \pm 0.2 \\
(2-4)\end{array}$ \\
\hline SWCA-3 & 11 & $\begin{array}{l}642 \pm 26.7 \\
(415-720)\end{array}$ & $\begin{array}{c}3692 \pm 358.9 \\
(1020-5256)\end{array}$ & $\begin{array}{l}607 \pm 84.5 \\
(115-1060)\end{array}$ & $\begin{array}{c}839 \pm 173.3 \\
(375-2309)\end{array}$ & $\begin{array}{c}1 \pm 0.2 \\
(0-3)\end{array}$ & $\begin{array}{c}21 \pm 4.6 \\
(2.347-56.932)\end{array}$ & $\begin{array}{c}3 \pm 0.2 \\
(1-4)\end{array}$ \\
\hline SWNV-1 & 19 & $\begin{array}{l}444 \pm 9.1 \\
(385-526)\end{array}$ & $\begin{array}{l}1237 \pm 72.5 \\
(719-1830)\end{array}$ & $\begin{array}{c}843 \pm 112.8 \\
(139-1766)\end{array}$ & $\begin{array}{l}424 \pm 65 \\
(112-992)\end{array}$ & $\begin{array}{c}2 \pm 0.3 \\
(0-6)\end{array}$ & $\begin{array}{c}1 \pm 0.1 \\
(0.15-2.423)\end{array}$ & $\begin{array}{c}3 \pm 0.3 \\
(0-4)\end{array}$ \\
\hline SWNV-2 & 18 & $\begin{array}{l}480 \pm 6.7 \\
(441-533)\end{array}$ & $\begin{array}{c}1568 \pm 91 \\
(1110-2462)\end{array}$ & $\begin{array}{l}944 \pm 85.1 \\
(163-1694)\end{array}$ & $\begin{array}{c}402 \pm 53.8 \\
(122-936)\end{array}$ & $\begin{array}{c}3 \pm 0.4 \\
(1-7)\end{array}$ & $\begin{array}{c}1 \pm 0.3 \\
(0.001-4.816)\end{array}$ & $\begin{array}{c}3 \pm 0.1 \\
(2-4)\end{array}$ \\
\hline
\end{tabular}


Table 3A. (Continued) Site and regional mean values for biomarker and ancillary data for COMMON CARP collections made at all sites in U.S. waters from March 1994 through August 1997.

[N, number of fish; E2, 17ß Estradiol; 11KT, 11 ketotestosterone; VTG, vitellogenin; mm, millimeter; g, grams; pg, picograms; mg, milligram; mL, milliliter; ND, nondetected]

\begin{tabular}{|c|c|c|c|c|c|c|c|c|}
\hline $\begin{array}{l}\text { Regional site } \\
\text { code } \\
\text { (fig. 1) }\end{array}$ & $\mathbf{N}$ & $\begin{array}{l}\text { Length } \\
\text { (mm) }\end{array}$ & $\begin{array}{l}\text { Weight } \\
\text { (g) }\end{array}$ & $\underset{(p g / m L)}{E_{2}}$ & $\begin{array}{c}11 \mathrm{KT} \\
(\mathrm{pg} / \mathrm{mL})\end{array}$ & $\begin{array}{l}\text { Ratio of } \\
E_{2} \text { to KT }\end{array}$ & $\underset{(\mathrm{mg} / \mathrm{mL})}{\mathrm{VTG}}$ & $\begin{array}{l}\text { Developmental } \\
\text { stage }\end{array}$ \\
\hline SWNV-3 & 24 & $\begin{array}{l}471 \pm 7.6 \\
(403-526)\end{array}$ & $\begin{array}{l}1427 \pm 64.4 \\
(745-2193)\end{array}$ & $\begin{array}{l}843 \pm 76.5 \\
(231-1401)\end{array}$ & $\begin{array}{c}311 \pm 33.9 \\
(56-707)\end{array}$ & $\begin{array}{l}4 \pm 0.7 \\
(1-16)\end{array}$ & $\begin{array}{c}3 \pm 0.7 \\
(0.001-16.124)\end{array}$ & $\begin{array}{c}3 \pm 0.1 \\
(2-3)\end{array}$ \\
\hline SWUT-5 & 1 & $\begin{array}{c}720 \pm 0 \\
(720-720)\end{array}$ & $\begin{array}{c}5000 \pm 0 \\
(5000-5000)\end{array}$ & $\begin{array}{c}1159 \pm 0 \\
(1159-1159)\end{array}$ & $\begin{array}{c}687 \pm 0 \\
(687-687)\end{array}$ & $\begin{array}{l}2 \pm 0 \\
(2-2)\end{array}$ & $\begin{array}{c}1 \pm 0 \\
(0.672-0.672)\end{array}$ & $\begin{array}{l}3 \pm 0 \\
(3-3)\end{array}$ \\
\hline Summary & 83 & $\begin{array}{l}487 \pm 9.1 \\
(385-720)\end{array}$ & $\begin{array}{c}1700 \pm 113.2 \\
(719-5256)\end{array}$ & $\begin{array}{l}790 \pm 44.1 \\
(115-1766)\end{array}$ & $\begin{array}{l}447 \pm 36 \\
(56-2309)\end{array}$ & $\begin{array}{l}3 \pm 0.3 \\
(0-16)\end{array}$ & $\begin{array}{c}4 \pm 0.9 \\
(0.001-56.932)\end{array}$ & $\begin{array}{c}3 \pm 0.1 \\
(0-4)\end{array}$ \\
\hline \multicolumn{9}{|c|}{$\square$ Male non-reproductive } \\
\hline SWCA-1 & 20 & $\begin{array}{c}477 \pm 10.8 \\
(416-566)\end{array}$ & $\begin{array}{c}1547 \pm 102.7 \\
(1009-2310)\end{array}$ & $\begin{array}{c}1338 \pm 220.2 \\
(88-2542)\end{array}$ & $\begin{array}{c}3010 \pm 385.9 \\
(152-5009)\end{array}$ & $\begin{array}{l}0 \pm 0 \\
(0-1)\end{array}$ & $\begin{array}{c}0 \pm 0 \\
(\mathrm{ND}-0.12)\end{array}$ & $\begin{array}{c}3 \pm 0.1 \\
(2-3)\end{array}$ \\
\hline SWCA-2 & 28 & $\begin{array}{l}455 \pm 18.7 \\
(302-640)\end{array}$ & $\begin{array}{c}1340 \pm 153.1 \\
(334-2905)\end{array}$ & $\begin{array}{c}414 \pm 98.8 \\
(51-2388)\end{array}$ & $\begin{array}{l}1988 \pm 460 \\
(183-9699)\end{array}$ & $\begin{array}{c}0 \pm 0.1 \\
(0-2)\end{array}$ & $\begin{array}{c}0 \pm 0 \\
(\mathrm{ND}-0.183)\end{array}$ & $\begin{array}{c}3 \pm 0.1 \\
(2-3)\end{array}$ \\
\hline SWCA-3 & 25 & $\begin{array}{c}533 \pm 13.1 \\
(420-660)\end{array}$ & $\begin{array}{c}2182 \pm 177.6 \\
(477-3806)\end{array}$ & $\begin{array}{c}894 \pm 205.1 \\
(51-4546)\end{array}$ & $\begin{array}{l}1531 \pm 276 \\
(149-4978)\end{array}$ & $\begin{array}{c}1 \pm 0.1 \\
(0-3)\end{array}$ & $\begin{array}{c}0 \pm 0.1 \\
(\mathrm{ND}-3.148)\end{array}$ & $\begin{array}{c}3 \pm 0.1 \\
(2-3)\end{array}$ \\
\hline SWUT-1 & 5 & $\begin{array}{c}472 \pm 59.5 \\
(336-674)\end{array}$ & $\begin{array}{c}1585 \pm 629.8 \\
(552-3983)\end{array}$ & $\begin{array}{l}629 \pm 55.5 \\
(531-841)\end{array}$ & $\begin{array}{c}875 \pm 119.3 \\
(575-1263)\end{array}$ & $\begin{array}{c}1 \pm 0.1 \\
(1-1)\end{array}$ & $\begin{array}{c}0 \pm 0 \\
(\mathrm{ND}-0.024)\end{array}$ & $\begin{array}{c}2 \pm 0.2 \\
(1-2)\end{array}$ \\
\hline SWUT-2 & 5 & $\begin{array}{c}455 \pm 42.9 \\
(347-570)\end{array}$ & $\begin{array}{c}1361 \pm 285.6 \\
(762-2168)\end{array}$ & $\begin{array}{l}286 \pm 62 \\
(168-468)\end{array}$ & $\begin{array}{c}776 \pm 212.8 \\
(347-1569)\end{array}$ & $\begin{array}{c}0 \pm 0.1 \\
(0-1)\end{array}$ & $\begin{array}{c}0 \pm 0 \\
(\mathrm{ND}-0.043)\end{array}$ & $\begin{array}{l}3 \pm 0 \\
(3-3)\end{array}$ \\
\hline SWUT-3 & 5 & $\begin{array}{c}559 \pm 77.4 \\
(391-779)\end{array}$ & $\begin{array}{l}2635 \pm 956 \\
(870-5700)\end{array}$ & $\begin{array}{l}638 \pm 97.2 \\
(281-835)\end{array}$ & $\begin{array}{c}1098 \pm 343.2 \\
(329-2324)\end{array}$ & $\begin{array}{c}1 \pm 0.1 \\
(0-1)\end{array}$ & $\begin{array}{c}0 \pm 0 \\
(\mathrm{ND}-0.019)\end{array}$ & $\begin{array}{c}2 \pm 0.4 \\
(1-3)\end{array}$ \\
\hline SWUT-4 & 4 & $\begin{array}{c}403 \pm 58.3 \\
(278-515)\end{array}$ & $\begin{array}{c}1506 \pm 432.7 \\
(473-2591)\end{array}$ & $\begin{array}{c}486 \pm 40.8 \\
(366-546)\end{array}$ & $\begin{array}{c}580 \pm 46.2 \\
(447-660)\end{array}$ & $\begin{array}{l}1 \pm 0 \\
(1-1)\end{array}$ & $\begin{array}{c}0 \pm 0 \\
(\mathrm{ND}-0.061)\end{array}$ & $\begin{array}{c}2 \pm 0.4 \\
(1-3)\end{array}$ \\
\hline SWUT-5 & 5 & $\begin{array}{l}465 \pm 30.1 \\
(380-552)\end{array}$ & $\begin{array}{c}1291 \pm 236.2 \\
(740-2060)\end{array}$ & $\begin{array}{c}504 \pm 68.8 \\
(263-690)\end{array}$ & $\begin{array}{c}944 \pm 149.1 \\
(521-1317)\end{array}$ & $\begin{array}{c}1 \pm 0.1 \\
(0-1)\end{array}$ & $\begin{array}{c}0 \pm 0 \\
(\mathrm{ND}-0.018)\end{array}$ & $\begin{array}{c}2 \pm 0.4 \\
(1-3)\end{array}$ \\
\hline Summary & 97 & $\begin{array}{l}485 \pm 9.5 \\
(278-779)\end{array}$ & $\begin{array}{c}1688 \pm 97.3 \\
(334-5700)\end{array}$ & $\begin{array}{c}752 \pm 82.8 \\
(51-4546)\end{array}$ & $\begin{array}{c}1804 \pm 185.4 \\
(149-9699)\end{array}$ & $\begin{array}{l}1 \pm 0 \\
(0-3)\end{array}$ & $\begin{array}{c}0 \pm 0 \\
(\mathrm{ND}-3.148)\end{array}$ & $\begin{array}{c}3 \pm 0.1 \\
(1-3)\end{array}$ \\
\hline \multicolumn{9}{|c|}{ Male reproductive } \\
\hline SWAZ-1 & 10 & $\begin{array}{l}430 \pm 7.9 \\
(404-490)\end{array}$ & $\begin{array}{c}887 \pm 53 \\
(717-1294)\end{array}$ & $\begin{array}{l}95 \pm 3.8 \\
(77-118)\end{array}$ & $\begin{array}{c}261 \pm 60.9 \\
(74-731)\end{array}$ & $\begin{array}{c}1 \pm 0.1 \\
(0-1)\end{array}$ & $\begin{array}{c}0 \pm 0.1 \\
(\mathrm{ND}-0.678)\end{array}$ & $\begin{array}{c}3 \pm 0.2 \\
(2-3)\end{array}$ \\
\hline SWCA-3 & 5 & $\begin{array}{c}600 \pm 17.9 \\
(544-649)\end{array}$ & $\begin{array}{c}2584 \pm 283.9 \\
(1923-3360)\end{array}$ & $\begin{array}{c}355 \pm 96.9 \\
(181-731)\end{array}$ & $\begin{array}{c}982 \pm 268.7 \\
(191-1866)\end{array}$ & $\begin{array}{c}1 \pm 0.3 \\
(0-1)\end{array}$ & $\begin{array}{c}0 \pm 0 \\
(\mathrm{ND}-0.037)\end{array}$ & $\begin{array}{c}3 \pm 0.4 \\
(1-3)\end{array}$ \\
\hline SWNV-1 & 20 & $\begin{array}{l}460 \pm 8.3 \\
(403-518)\end{array}$ & $\begin{array}{l}1236 \pm 70.6 \\
(745-1830)\end{array}$ & $\begin{array}{c}404 \pm 93.4 \\
(71-1298)\end{array}$ & $\begin{array}{c}638 \pm 127.3 \\
(127-2481)\end{array}$ & $\begin{array}{c}1 \pm 0.1 \\
(0-2)\end{array}$ & $\begin{array}{c}0 \pm 0 \\
(\mathrm{ND}-0.005)\end{array}$ & $\begin{array}{c}3 \pm 0.2 \\
(1-3)\end{array}$ \\
\hline SWNV-2 & 19 & $\begin{array}{l}469 \pm 7.9 \\
(393-538)\end{array}$ & $\begin{array}{c}1306 \pm 72.7 \\
(666-1885)\end{array}$ & $\begin{array}{c}180 \pm 30.9 \\
(63-519)\end{array}$ & $\begin{array}{l}545 \pm 83.3 \\
(109-1363)\end{array}$ & $\begin{array}{l}0 \pm 0 \\
(0-1)\end{array}$ & $\begin{array}{c}0 \pm 0 \\
(\mathrm{ND}-0.233)\end{array}$ & $\begin{array}{c}3 \pm 0.1 \\
(2-3)\end{array}$ \\
\hline SWNV-3 & 22 & $\begin{array}{l}467 \pm 8.3 \\
(378-526)\end{array}$ & $\begin{array}{c}1542 \pm 79 \\
(1033-2193)\end{array}$ & $\begin{array}{c}282 \pm 27.1 \\
(71-585)\end{array}$ & $\begin{array}{c}1279 \pm 166.4 \\
(257-2804)\end{array}$ & $\begin{array}{l}0 \pm 0 \\
(0-1)\end{array}$ & $\begin{array}{c}0 \pm 0 \\
(0.001-0.128)\end{array}$ & $\begin{array}{c}3 \pm 0.1 \\
(2-3)\end{array}$ \\
\hline Summary & 76 & $\begin{array}{l}470 \pm 5.8 \\
(378-649)\end{array}$ & $\begin{array}{c}1385 \pm 58.1 \\
(666-3360)\end{array}$ & $\begin{array}{l}269 \pm 29.7 \\
(63-1298)\end{array}$ & $\begin{array}{c}773 \pm 76 \\
(74-2804)\end{array}$ & $\begin{array}{l}0 \pm 0 \\
(0-2)\end{array}$ & $\begin{array}{c}0 \pm 0 \\
(\mathrm{ND}-0.678)\end{array}$ & $\begin{array}{c}3 \pm 0.1 \\
(1-3)\end{array}$ \\
\hline
\end{tabular}


Table 3B. Site and regional mean values for biomarker and ancillary data for LARGEMOUTH BASS collections made at all sites in U.S. waters from March 1994 through August 1997.

[N, number of fish; E2, 17ß Estradiol; 11KT, 11 ketotestosterone; VTG, vitellogenin; mm, millimeter; g, grams; pg, picograms; mg, milligram; mL, milliliter; $\mathrm{ND}$, nondetected]

\begin{tabular}{|c|c|c|c|c|c|c|c|c|}
\hline $\begin{array}{c}\text { Regional site } \\
\text { code } \\
\text { (fig. 1) }\end{array}$ & $\mathbf{N}$ & $\begin{array}{l}\text { Length } \\
\text { (mm) }\end{array}$ & $\begin{array}{l}\text { Weight } \\
\text { (g) }\end{array}$ & $\underset{(p g / m L)}{E_{2}}$ & $\begin{array}{c}11 \mathrm{KT} \\
\text { (pg/mL) }\end{array}$ & $\begin{array}{l}\text { Ratio of } \\
E_{2} \text { to KT }\end{array}$ & $\underset{(\mathrm{mg} / \mathrm{mL})}{\mathrm{VTG}}$ & $\begin{array}{l}\text { Developmental } \\
\text { stage }\end{array}$ \\
\hline
\end{tabular}

\section{LARGEMOUTH BASS}

Northern Mid-Continent Region

\section{Female non-reproductive}

\begin{tabular}{|c|c|c|c|c|c|c|c|c|}
\hline NMCO-3 & 8 & $\begin{array}{l}332 \pm 4.3 \\
(309-348)\end{array}$ & $\begin{array}{l}741 \pm 32.7 \\
(600-850)\end{array}$ & $\begin{array}{l}977 \pm 193.8 \\
(403-1883)\end{array}$ & $\begin{array}{c}369 \pm 107.2 \\
(111-1040)\end{array}$ & $\begin{array}{l}3 \pm 0.3 \\
(2-4.3)\end{array}$ & $\begin{array}{c}1 \pm 1.1 \\
(\mathrm{ND}-8.833)\end{array}$ & $\begin{array}{l}2 \pm 0 \\
(2-2)\end{array}$ \\
\hline NMIA-7 & 10 & $\begin{array}{l}382 \pm 7.3 \\
(355-420)\end{array}$ & $\begin{array}{l}972 \pm 59.2 \\
(682-1272)\end{array}$ & $\begin{array}{l}750 \pm 46.9 \\
(604-1116)\end{array}$ & $\begin{array}{l}558 \pm 46.5 \\
(304-771)\end{array}$ & $\begin{array}{l}1 \pm 0.2 \\
(1-2.3)\end{array}$ & $\begin{array}{c}3 \pm 1.1 \\
(0.116-11.276)\end{array}$ & $\begin{array}{c}2 \pm 0.1 \\
(2-3)\end{array}$ \\
\hline NMIA-8 & 10 & $\begin{array}{c}398 \pm 10.4 \\
(337-456)\end{array}$ & $\begin{array}{l}1102 \pm 96.3 \\
(656-1726)\end{array}$ & $\begin{array}{l}997 \pm 150.3 \\
(202-1371)\end{array}$ & $\begin{array}{l}406 \pm 66.5 \\
(179-782)\end{array}$ & $\begin{array}{l}3 \pm 0.4 \\
(1-4.6)\end{array}$ & $\begin{array}{c}2 \pm 1 \\
(0.021-10.331)\end{array}$ & $\begin{array}{l}2 \pm 0 \\
(2-2)\end{array}$ \\
\hline NMIL-1 & 10 & $\begin{array}{l}319 \pm 19.3 \\
(251-408)\end{array}$ & $\begin{array}{l}618 \pm 117.6 \\
(228-1173)\end{array}$ & $\begin{array}{c}236 \pm 26.6 \\
(102-423)\end{array}$ & $\begin{array}{c}473 \pm 95.9 \\
(128-906)\end{array}$ & $\begin{array}{l}1 \pm 0.2 \\
(0-1.6)\end{array}$ & $\begin{array}{c}0 \pm 0 \\
(\mathrm{ND}-0.072)\end{array}$ & $\begin{array}{c}2 \pm 0.1 \\
(1-3)\end{array}$ \\
\hline NMIN-4 & 4 & $\begin{array}{l}297 \pm 11.2 \\
(266-316)\end{array}$ & $\begin{array}{c}340 \pm 59.4 \\
(176-450)\end{array}$ & $\begin{array}{l}681 \pm 249.8 \\
(369-1426)\end{array}$ & $\begin{array}{c}173 \pm 55.1 \\
(97-337)\end{array}$ & $\begin{array}{l}4 \pm 0.5 \\
(3-5.1)\end{array}$ & $\begin{array}{c}1 \pm 1.3 \\
(\mathrm{ND}-5.092)\end{array}$ & $\begin{array}{c}2 \pm 0.6 \\
(0-3)\end{array}$ \\
\hline NMMO-3 & 5 & $\begin{array}{c}339 \pm 25.2 \\
(296-426)\end{array}$ & $\begin{array}{l}645 \pm 157.6 \\
(375-1225)\end{array}$ & $\begin{array}{c}494 \pm 77.7 \\
(338-789)\end{array}$ & $\begin{array}{l}369 \pm 76.6 \\
(176-622)\end{array}$ & $\begin{array}{l}2 \pm 0.3 \\
(1-2.4)\end{array}$ & $\begin{array}{c}0 \pm 0.2 \\
(\mathrm{ND}-1.21)\end{array}$ & $\begin{array}{l}2 \pm 0 \\
(2-2)\end{array}$ \\
\hline Summary & 47 & $\begin{array}{l}352 \pm 7.4 \\
(251-456)\end{array}$ & $\begin{array}{l}796 \pm 50.6 \\
(176-1726)\end{array}$ & $\begin{array}{l}699 \pm 65.1 \\
(102-1883)\end{array}$ & $\begin{array}{l}422 \pm 35.4 \\
(97-1040)\end{array}$ & $\begin{array}{l}2 \pm 0.2 \\
(0-5.1)\end{array}$ & $\begin{array}{c}1 \pm 0.4 \\
(\mathrm{ND}-11.276)\end{array}$ & $\begin{array}{c}2 \pm 0.1 \\
(0-3)\end{array}$ \\
\hline \multicolumn{9}{|c|}{$\square$ Male non-reproductive } \\
\hline NMCO-3 & 10 & $\begin{array}{l}331 \pm 4.7 \\
(295-349)\end{array}$ & $\begin{array}{l}759 \pm 31.5 \\
(550-875)\end{array}$ & $\begin{array}{l}448 \pm 28.4 \\
(329-544)\end{array}$ & $\begin{array}{c}1369 \pm 237.5 \\
(561-2812)\end{array}$ & $\begin{array}{l}0 \pm 0.1 \\
(0-0.8)\end{array}$ & $\begin{array}{c}0 \pm 0 \\
(\mathrm{ND}-0.001)\end{array}$ & $\begin{array}{l}2 \pm 0 \\
(2-2)\end{array}$ \\
\hline NMIA-7 & 10 & $\begin{array}{l}337 \pm 9.6 \\
(275-387)\end{array}$ & $\begin{array}{l}661 \pm 59.6 \\
(323-1030)\end{array}$ & $\begin{array}{l}551 \pm 45.4 \\
(340-857)\end{array}$ & $\begin{array}{l}982 \pm 87.3 \\
(432-1335)\end{array}$ & $\begin{array}{l}1 \pm 0.1 \\
(0-1.2)\end{array}$ & $\begin{array}{c}0 \pm 0 \\
(\mathrm{ND}-0.026)\end{array}$ & $\begin{array}{c}2 \pm 0.2 \\
(1-2)\end{array}$ \\
\hline NMIA-8 & 10 & $\begin{array}{l}367 \pm 7.8 \\
(310-393)\end{array}$ & $\begin{array}{l}817 \pm 53.8 \\
(449-1008)\end{array}$ & $\begin{array}{c}220 \pm 20.3 \\
(99-291)\end{array}$ & $\begin{array}{l}654 \pm 46.2 \\
(386-901)\end{array}$ & $\begin{array}{l}0 \pm 0.1 \\
(0-0.8)\end{array}$ & $\begin{array}{c}0 \pm 0 \\
(\mathrm{ND}-0.006)\end{array}$ & $\begin{array}{c}2 \pm 0.1 \\
(1-2)\end{array}$ \\
\hline NMIL-1 & 10 & $\begin{array}{c}310 \pm 17.3 \\
(234-404)\end{array}$ & $\begin{array}{l}539 \pm 101.2 \\
(192-1119)\end{array}$ & $\begin{array}{c}201 \pm 11.8 \\
(153-251)\end{array}$ & $\begin{array}{l}750 \pm 172.2 \\
(231-1755)\end{array}$ & $\begin{array}{c}0 \pm 0.1 \\
(0-1)\end{array}$ & $\begin{array}{c}0 \pm 0 \\
(\mathrm{ND}-0.001)\end{array}$ & $\begin{array}{c}1 \pm 0.1 \\
(1-2)\end{array}$ \\
\hline NMIN-4 & 5 & $\begin{array}{l}301 \pm 10 \\
(265-318)\end{array}$ & $\begin{array}{l}385 \pm 44.5 \\
(258-500)\end{array}$ & $\begin{array}{l}566 \pm 197.8 \\
(315-1355)\end{array}$ & $\begin{array}{c}717 \pm 334.5 \\
(129-1681)\end{array}$ & $\begin{array}{l}2 \pm 1.1 \\
(0-6.2)\end{array}$ & $\begin{array}{c}0 \pm 0 \\
(\mathrm{ND}-0.008)\end{array}$ & $\begin{array}{c}2 \pm 0.2 \\
(2-3)\end{array}$ \\
\hline NMMO-3 & 4 & $\begin{array}{l}349 \pm 31.8 \\
(287-435)\end{array}$ & $\begin{array}{l}800 \pm 229.8 \\
(400-1450)\end{array}$ & $\begin{array}{c}648 \pm 108.4 \\
(395-923)\end{array}$ & $\begin{array}{c}1020 \pm 438.7 \\
(191-2132)\end{array}$ & $\begin{array}{l}1 \pm 0.4 \\
(0-2.1)\end{array}$ & $\begin{array}{c}0 \pm 0 \\
(\mathrm{ND}-0.001)\end{array}$ & $\begin{array}{l}2 \pm 0 \\
(2-2)\end{array}$ \\
\hline Summary & 49 & $\begin{array}{l}334 \pm 5.8 \\
(234-435)\end{array}$ & $\begin{array}{l}671 \pm 36.9 \\
(192-1450)\end{array}$ & $\begin{array}{l}400 \pm 33.1 \\
(99-1355)\end{array}$ & $\begin{array}{l}923 \pm 83.7 \\
(129-2812)\end{array}$ & $\begin{array}{l}1 \pm 0.1 \\
(0-6.2)\end{array}$ & $\begin{array}{c}0 \pm 0 \\
(\mathrm{ND}-0.026)\end{array}$ & $\begin{array}{c}2 \pm 0.1 \\
(1-3)\end{array}$ \\
\hline
\end{tabular}

\section{Female non-reproductive}

$\begin{array}{lccccccrr}\text { NECT-1 } & 3 & 295 \pm 39.2 & 446 \pm 169.2 & 730 \pm 326.5 & 133 \pm 31.4 & 5 \pm 1.1 & 0 \pm 0 & (1-0) \\ & & (254-373) & (273-784) & (307-1372) & (93-195) & (3-7) & (\text { ND- } 0.008) & 1 \pm 1 \\ \text { NEDC-1 } & 5 & 340 \pm 22.9 & 597 \pm 148.9 & 228 \pm 84.9 & 267 \pm 122.5 & 1 \pm 0.2 & 0 \pm 0.2 & (1-0) \\ & & (293-400) & (354-1164) & (51-546) & (35-731) & (1-1.8) & (0.0016-0.88) & 1 \pm 1\end{array}$


Table 3B. (Continued) Site and regional mean values for biomarker and ancillary data for LARGEMOUTH BASS collections made at all sites in U.S. waters from March 1994 through August 1997.

[N, number of fish; E2, 17ß Estradiol; 11KT, 11 ketotestosterone; VTG, vitellogenin; mm, millimeter; g, grams; pg, picograms; mg, milligram; mL, milliliter; ND, nondetected]

\begin{tabular}{|c|c|c|c|c|c|c|c|c|}
\hline $\begin{array}{c}\text { Regional site } \\
\text { code } \\
\text { (fig. 1) }\end{array}$ & $\mathbf{N}$ & $\begin{array}{l}\text { Length } \\
\text { (mm) }\end{array}$ & $\begin{array}{l}\text { Weight } \\
\text { (g) }\end{array}$ & $\underset{(p g / m L)}{E_{2}}$ & $\begin{array}{c}11 \mathrm{KT} \\
(\mathrm{pg} / \mathrm{mL})\end{array}$ & $\begin{array}{l}\text { Ratio of } \\
E_{2} \text { to KT }\end{array}$ & $\underset{(\mathrm{mg} / \mathrm{mL})}{\mathrm{VTG}}$ & $\begin{array}{l}\text { Developmental } \\
\text { stage }\end{array}$ \\
\hline NEMA -1 & 12 & $\begin{array}{c}310 \pm 9 \\
(264-375)\end{array}$ & $\begin{array}{c}479 \pm 51.5 \\
(288-886)\end{array}$ & $\begin{array}{c}387 \pm 85.4 \\
(33-958)\end{array}$ & $\begin{array}{c}376 \pm 63.3 \\
(123-729)\end{array}$ & $\begin{array}{l}1 \pm 0.3 \\
(0-2.5)\end{array}$ & $\begin{array}{c}0 \pm 0 \\
(0.0008-0.008)\end{array}$ & $\begin{array}{l}(1-0) \\
1 \pm 1\end{array}$ \\
\hline NENY-2 & 4 & $\begin{array}{l}307 \pm 1.9 \\
(303-312)\end{array}$ & $\begin{array}{c}478 \pm 21.4 \\
(430-534)\end{array}$ & $\begin{array}{l}375 \pm 99.7 \\
(172-561)\end{array}$ & $\begin{array}{c}118 \pm 84.6 \\
(18-371)\end{array}$ & $\begin{array}{c}9 \pm 3.9 \\
(2-17.7)\end{array}$ & $\begin{array}{c}0 \pm 0.1 \\
(\mathrm{ND}-0.4)\end{array}$ & $\begin{array}{l}(1-0) \\
1 \pm 1\end{array}$ \\
\hline NENY-3 & 7 & $\begin{array}{l}413 \pm 18.9 \\
(359-495)\end{array}$ & $\begin{array}{l}890 \pm 53.2 \\
(785-956)\end{array}$ & $\begin{array}{l}444 \pm 87.1 \\
(124-741)\end{array}$ & $\begin{array}{c}116 \pm 25.1 \\
(46-244)\end{array}$ & $\begin{array}{l}5 \pm 1.8 \\
(1-15.2)\end{array}$ & $\begin{array}{c}0 \pm 0 \\
(0.006-0.4)\end{array}$ & $\begin{array}{c}(2-0.2) \\
1 \pm 2\end{array}$ \\
\hline NENY-4 & 2 & $\begin{array}{c}439 \pm 45.5 \\
(393-484)\end{array}$ & $\begin{array}{c}1414 \pm 399 \\
(1015-1813)\end{array}$ & $\begin{array}{l}350 \pm 92.5 \\
(257-442)\end{array}$ & $\begin{array}{c}97 \pm 76 \\
(21-173)\end{array}$ & $\begin{array}{c}7 \pm 4.8 \\
(3-12.2)\end{array}$ & $\begin{array}{c}0 \pm 0 \\
(0.097-0.139)\end{array}$ & $\begin{array}{l}(1-0) \\
1 \pm 1\end{array}$ \\
\hline NEOH-1 & 2 & $\begin{array}{c}330 \pm 2 \\
(328-332)\end{array}$ & $\begin{array}{c}602 \pm 38 \\
(564-640)\end{array}$ & $\begin{array}{l}322 \pm 42.5 \\
(279-364)\end{array}$ & $\begin{array}{l}119 \pm 32 \\
(87-151)\end{array}$ & $\begin{array}{l}3 \pm 0.4 \\
(2-3.2)\end{array}$ & $\begin{array}{c}1 \pm 0.4 \\
(0.245-1.003)\end{array}$ & $\begin{array}{l}(3-0) \\
3 \pm 3\end{array}$ \\
\hline NEWV -2 & 10 & $\begin{array}{l}308 \pm 4.3 \\
(284-325)\end{array}$ & $\begin{array}{l}352 \pm 15.1 \\
(278-418.2)\end{array}$ & $\begin{array}{l}683 \pm 121 \\
(304-1403)\end{array}$ & $\begin{array}{c}335 \pm 60.5 \\
(84-601)\end{array}$ & $\begin{array}{l}2 \pm 0.3 \\
(1-3.6)\end{array}$ & $\begin{array}{c}0 \pm 0.1 \\
(\mathrm{ND}-0.512)\end{array}$ & $\begin{array}{c}(2-0.1) \\
1 \pm 2\end{array}$ \\
\hline Summary & 45 & $\begin{array}{l}334 \pm 8.3 \\
(254-495)\end{array}$ & $\begin{array}{l}541 \pm 47.4 \\
(273-1813)\end{array}$ & $\begin{array}{c}461 \pm 48.5 \\
(33-1403)\end{array}$ & $\begin{array}{c}251 \pm 30.7 \\
(18-731)\end{array}$ & $\begin{array}{c}3 \pm 0.6 \\
(0-17.7)\end{array}$ & $\begin{array}{c}0 \pm 0 \\
(\mathrm{ND}-1.003)\end{array}$ & $\begin{array}{c}(1-0.1) \\
1 \pm 3\end{array}$ \\
\hline \multicolumn{9}{|c|}{$\square$ Male non-reproductive } \\
\hline NECT-1 & 4 & $\begin{array}{c}314 \pm 26.5 \\
(284-393)\end{array}$ & $\begin{array}{c}389 \pm 37.8 \\
(314-494)\end{array}$ & $\begin{array}{l}709 \pm 282.6 \\
(231-1500)\end{array}$ & $\begin{array}{c}757 \pm 259.4 \\
(257-1206)\end{array}$ & $\begin{array}{l}1 \pm 0.2 \\
(1-1.5)\end{array}$ & $\begin{array}{c}0 \pm 0 \\
(\mathrm{ND}-0.002)\end{array}$ & $\begin{array}{l}3 \pm 0 \\
(3-3)\end{array}$ \\
\hline NEDC-1 & 7 & $\begin{array}{c}339 \pm 13.3 \\
(274-386)\end{array}$ & $\begin{array}{c}581 \pm 65.5 \\
(228-780)\end{array}$ & $\begin{array}{l}121 \pm 38 \\
(21-294)\end{array}$ & $\begin{array}{c}129 \pm 42.2 \\
(46-327)\end{array}$ & $\begin{array}{l}1 \pm 0.4 \\
(0-3.7)\end{array}$ & $\begin{array}{c}0 \pm 0 \\
(0.001-0.002)\end{array}$ & $\begin{array}{l}3 \pm 0 \\
(3-3)\end{array}$ \\
\hline NEMA -1 & 7 & $\begin{array}{l}296 \pm 10.7 \\
(251-335)\end{array}$ & $\begin{array}{l}393 \pm 42.2 \\
(248-542)\end{array}$ & $\begin{array}{c}526 \pm 122.3 \\
(107-1054)\end{array}$ & $\begin{array}{c}383 \pm 85.1 \\
(176-807)\end{array}$ & $\begin{array}{l}2 \pm 0.5 \\
(0-4.2)\end{array}$ & $\begin{array}{c}0 \pm 0 \\
(0.001-0.004)\end{array}$ & $\begin{array}{c}2 \pm 0.4 \\
(0-3)\end{array}$ \\
\hline NENY-2 & 6 & $\begin{array}{l}292 \pm 5.2 \\
(277-310)\end{array}$ & $\begin{array}{l}378 \pm 15 \\
(325-420)\end{array}$ & $\begin{array}{c}200 \pm 44.7 \\
(44-373)\end{array}$ & $\begin{array}{l}68 \pm 15.2 \\
(39-142)\end{array}$ & $\begin{array}{l}3 \pm 0.8 \\
(1-6.6)\end{array}$ & $\begin{array}{c}0 \pm 0 \\
(0.001-0.215)\end{array}$ & $\begin{array}{l}3 \pm 0 \\
(3-3)\end{array}$ \\
\hline NENY-3 & 12 & $\begin{array}{c}339 \pm 15.4 \\
(252-422)\end{array}$ & $\begin{array}{c}716 \pm 134.5 \\
(581-850)\end{array}$ & $\begin{array}{l}60 \pm 18.9 \\
(13-200)\end{array}$ & $\begin{array}{l}151 \pm 31 \\
(63-446)\end{array}$ & $\begin{array}{c}0 \pm 0.1 \\
(0-1)\end{array}$ & $\begin{array}{l}0 \pm 0.2 \\
(\mathrm{ND}-3)\end{array}$ & $\begin{array}{l}3 \pm 0 \\
(3-3)\end{array}$ \\
\hline NENY-4 & 2 & $\begin{array}{c}377 \pm 7 \\
(370-384)\end{array}$ & $\begin{array}{l}874 \pm 19 \\
(855-893)\end{array}$ & $\begin{array}{c}35 \pm 23.5 \\
(11-58)\end{array}$ & $\begin{array}{c}41 \pm 25.5 \\
(15-66)\end{array}$ & $\begin{array}{l}2 \pm 1.9 \\
(0-3.9)\end{array}$ & $\begin{array}{c}0 \pm 0 \\
(\mathrm{ND}-0.001)\end{array}$ & $\begin{array}{l}3 \pm 0 \\
(3-3)\end{array}$ \\
\hline NEOH-1 & 2 & $\begin{array}{c}395 \pm 15.5 \\
(379-410)\end{array}$ & $\begin{array}{c}945 \pm 155.5 \\
(789-1100)\end{array}$ & $\begin{array}{l}359 \pm 50.5 \\
(308-409)\end{array}$ & $\begin{array}{c}300 \pm 141.5 \\
(158-441)\end{array}$ & $\begin{array}{l}2 \pm 0.9 \\
(1-2.6)\end{array}$ & $\begin{array}{c}0 \pm 0 \\
(\mathrm{ND}-0.001)\end{array}$ & $\begin{array}{l}2 \pm 0 \\
(2-2)\end{array}$ \\
\hline NEWV -2 & 10 & $\begin{array}{l}300 \pm 6.9 \\
(263-330)\end{array}$ & $\begin{array}{c}294 \pm 16.6 \\
(214-377.8)\end{array}$ & $\begin{array}{c}313 \pm 16.4 \\
(201-387)\end{array}$ & $\begin{array}{l}748 \pm 64.7 \\
(426-1087)\end{array}$ & $\begin{array}{c}0 \pm 0 \\
(0-0.6)\end{array}$ & $\begin{array}{c}0 \pm 0 \\
(\mathrm{ND}-0.114)\end{array}$ & $\begin{array}{c}2 \pm 0.1 \\
(1-3)\end{array}$ \\
\hline Summary & 50 & $\begin{array}{l}321 \pm 6.2 \\
(251-422)\end{array}$ & $\begin{array}{c}466 \pm 34 \\
(214-1100)\end{array}$ & $\begin{array}{c}264 \pm 39.8 \\
(11-1500)\end{array}$ & $\begin{array}{c}340 \pm 47.3 \\
(15-1206)\end{array}$ & $\begin{array}{l}1 \pm 0.2 \\
(0-6.6)\end{array}$ & $\begin{array}{l}0 \pm 0.1 \\
(\mathrm{ND}-3)\end{array}$ & $\begin{array}{c}3 \pm 0.1 \\
(0-3)\end{array}$ \\
\hline \multicolumn{9}{|c|}{ Female reproductive } \\
\hline NEMA -1 & 4 & $\begin{array}{l}303 \pm 7.9 \\
(286-318)\end{array}$ & $\begin{array}{c}420 \pm 39.4 \\
(350-497)\end{array}$ & $\begin{array}{c}920 \pm 120.6 \\
(732-1243)\end{array}$ & $\begin{array}{l}531 \pm 92.8 \\
(382-784)\end{array}$ & $\begin{array}{l}2 \pm 0.1 \\
(2-1.9)\end{array}$ & $\begin{array}{c}4 \pm 2 \\
(\mathrm{ND}-8.242)\end{array}$ & $\begin{array}{c}3 \pm 0.4 \\
(2-4)\end{array}$ \\
\hline NENY-3 & 14 & $\begin{array}{c}399 \pm 12.5 \\
(325-478)\end{array}$ & $\begin{array}{c}1001 \pm 99.8 \\
(478-1648)\end{array}$ & $\begin{array}{c}3221 \pm 725.6 \\
(384-9670)\end{array}$ & $\begin{array}{c}754 \pm 196.4 \\
(108-2306)\end{array}$ & $\begin{array}{l}5 \pm 0.7 \\
(2-12.4)\end{array}$ & $\begin{array}{c}5 \pm 0.9 \\
(\mathrm{ND}-12.441)\end{array}$ & $\begin{array}{c}3 \pm 0.2 \\
(3-5)\end{array}$ \\
\hline Summary & 18 & $\begin{array}{l}378 \pm 13.7 \\
(286-478)\end{array}$ & $\begin{array}{l}872 \pm 97.1 \\
(350-1648)\end{array}$ & $\begin{array}{c}2710 \pm 606.2 \\
(384-9670)\end{array}$ & $\begin{array}{c}704 \pm 154.3 \\
(108-2306)\end{array}$ & $\begin{array}{c}5 \pm 0.7 \\
(2-12.4)\end{array}$ & $\begin{array}{c}5 \pm 0.8 \\
(\mathrm{ND}-12.441)\end{array}$ & $\begin{array}{c}3 \pm 0.1 \\
(2-5)\end{array}$ \\
\hline
\end{tabular}


Table 3B. (Continued) Site and regional mean values for biomarker and ancillary data for LARGEMOUTH BASS collections made at all sites in U.S. waters from March 1994 through August 1997.

[N, number of fish; E2, 17ß Estradiol; 11KT, 11 ketotestosterone; VTG, vitellogenin; mm, millimeter; g, grams; pg, picograms; mg, milligram; mL, milliliter; $\mathrm{ND}$, nondetected]

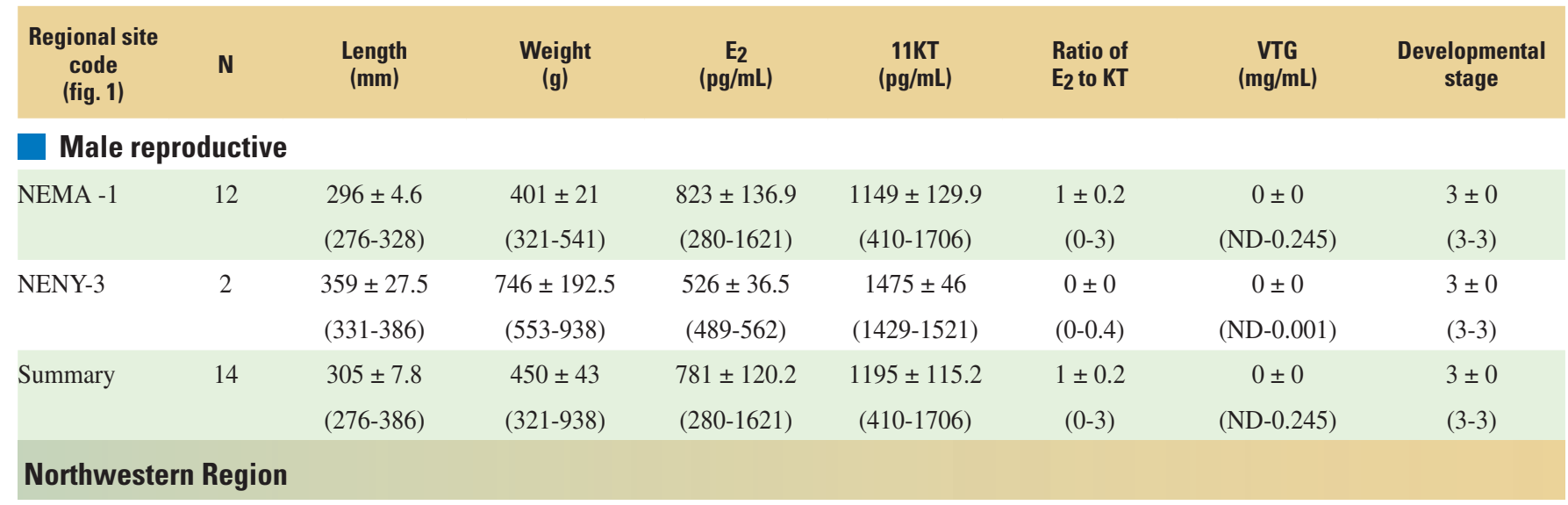

\section{Female non-reproductive}

\begin{tabular}{|c|c|c|c|c|c|c|c|c|}
\hline NWID-1 & 9 & $\begin{array}{l}365 \pm 22.8 \\
(257-492)\end{array}$ & $\begin{array}{c}891 \pm 170.3 \\
(253-2000)\end{array}$ & $\begin{array}{l}642 \pm 91.4 \\
(242-1134)\end{array}$ & $\begin{array}{c}287 \pm 42.2 \\
(167-481)\end{array}$ & $\begin{array}{l}2 \pm 0.4 \\
(1-4.2)\end{array}$ & $\begin{array}{c}0 \pm 0.2 \\
(0.026-1.535)\end{array}$ & $\begin{array}{c}1 \pm 0.1 \\
(1-2)\end{array}$ \\
\hline NWMT-1 & 10 & $\begin{array}{l}407 \pm 25.7 \\
(279-496)\end{array}$ & $\begin{array}{c}1320 \pm 249.3 \\
(280-2600)\end{array}$ & $\begin{array}{c}855 \pm 125.4 \\
(398-1585)\end{array}$ & $\begin{array}{c}383 \pm 67.3 \\
(112-805)\end{array}$ & $\begin{array}{l}2 \pm 0.2 \\
(2-3.7)\end{array}$ & $\begin{array}{c}1 \pm 0.2 \\
(0.158-2.355)\end{array}$ & $\begin{array}{c}2 \pm 0.2 \\
(1-3)\end{array}$ \\
\hline NWOR-1 & 5 & $\begin{array}{c}328 \pm 13.2 \\
(280-355)\end{array}$ & $\begin{array}{l}571 \pm 84.7 \\
(306-758)\end{array}$ & $\begin{array}{l}588 \pm 80.5 \\
(412-797)\end{array}$ & $\begin{array}{c}266 \pm 75.6 \\
(101-532)\end{array}$ & $\begin{array}{l}3 \pm 0.8 \\
(1-5.7)\end{array}$ & $\begin{array}{c}0 \pm 0 \\
(\mathrm{ND}-0.138)\end{array}$ & $\begin{array}{l}1 \pm 0 \\
(1-1)\end{array}$ \\
\hline NWWA-3 & 7 & $\begin{array}{l}355 \pm 32.9 \\
(269-522)\end{array}$ & $\begin{array}{c}920 \pm 316.7 \\
(299-2600)\end{array}$ & $\begin{array}{l}684 \pm 157.6 \\
(239-1465)\end{array}$ & $\begin{array}{c}360 \pm 110.5 \\
(122-908)\end{array}$ & $\begin{array}{l}2 \pm 0.5 \\
(1-4.5)\end{array}$ & $\begin{array}{c}5 \pm 4.8 \\
(\mathrm{ND}-33.665)\end{array}$ & $\begin{array}{c}1 \pm 0.3 \\
(1-3)\end{array}$ \\
\hline NWWA-6 & 4 & $\begin{array}{c}363 \pm 40.8 \\
(263-450)\end{array}$ & $\begin{array}{c}944 \pm 329.2 \\
(272-1803)\end{array}$ & $\begin{array}{l}1123 \pm 164 \\
(775-1406)\end{array}$ & $\begin{array}{c}404 \pm 121.2 \\
(193-689)\end{array}$ & $\begin{array}{l}3 \pm 0.5 \\
(2-4.3)\end{array}$ & $\begin{array}{c}1 \pm 0.5 \\
(0.299-2.631)\end{array}$ & $\begin{array}{c}2 \pm 0.3 \\
(1-2)\end{array}$ \\
\hline Summary & 35 & $\begin{array}{l}369 \pm 12.7 \\
(257-522)\end{array}$ & $\begin{array}{c}980 \pm 113.9 \\
(253-2600)\end{array}$ & $\begin{array}{l}758 \pm 61.1 \\
(239-1585)\end{array}$ & $\begin{array}{c}340 \pm 34.7 \\
(101-908)\end{array}$ & $\begin{array}{l}3 \pm 0.2 \\
(1-5.7)\end{array}$ & $\begin{array}{c}2 \pm 1 \\
(\mathrm{ND}-33.665)\end{array}$ & $\begin{array}{c}1 \pm 0.1 \\
(1-3)\end{array}$ \\
\hline
\end{tabular}

\section{Male non-reproductive}

\begin{tabular}{|c|c|c|c|c|c|c|c|c|}
\hline NWID-1 & 7 & $\begin{array}{c}344 \pm 12.6 \\
(314-397)\end{array}$ & $\begin{array}{c}673 \pm 79.4 \\
(444-970)\end{array}$ & $\begin{array}{l}341 \pm 98 \\
(131-815)\end{array}$ & $\begin{array}{c}1540 \pm 987.6 \\
(349-7451)\end{array}$ & $\begin{array}{c}1 \pm 0.2 \\
(0-2)\end{array}$ & $\begin{array}{c}0 \pm 0 \\
(\mathrm{ND}-0.001)\end{array}$ & $\begin{array}{c}2 \pm 0.3 \\
(1-3)\end{array}$ \\
\hline NWMT-1 & 10 & $\begin{array}{c}339 \pm 22.3 \\
(252-461)\end{array}$ & $\begin{array}{l}756 \pm 198.3 \\
(114-1880)\end{array}$ & $\begin{array}{l}251 \pm 46.4 \\
(113-591)\end{array}$ & $\begin{array}{l}528 \pm 88.6 \\
(121-976)\end{array}$ & $\begin{array}{l}1 \pm 0.2 \\
(0-2.3)\end{array}$ & $\begin{array}{c}0 \pm 0 \\
(\mathrm{ND}-0.083)\end{array}$ & $\begin{array}{c}3 \pm 0.2 \\
(1-3)\end{array}$ \\
\hline NWOR-1 & 3 & $\begin{array}{l}286 \pm 25.1 \\
(255-336)\end{array}$ & $\begin{array}{c}373 \pm 110.9 \\
(224-590)\end{array}$ & $\begin{array}{l}641 \pm 210.2 \\
(310-1031)\end{array}$ & $\begin{array}{c}813 \pm 354.3 \\
(260-1473)\end{array}$ & $\begin{array}{c}2 \pm 1.2 \\
(0-4)\end{array}$ & $\begin{array}{c}0 \pm 0 \\
(\mathrm{ND}-0.001)\end{array}$ & $\begin{array}{c}3 \pm 0.3 \\
(2-3)\end{array}$ \\
\hline NWWA-3 & 6 & $\begin{array}{l}314 \pm 35.7 \\
(240-440)\end{array}$ & $\begin{array}{l}534 \pm 194.5 \\
(144-1248)\end{array}$ & $\begin{array}{c}376 \pm 107.2 \\
(131-848)\end{array}$ & $\begin{array}{c}661 \pm 143.3 \\
(331-1307)\end{array}$ & $\begin{array}{l}1 \pm 0.1 \\
(0-1.2)\end{array}$ & $\begin{array}{c}0 \pm 0.1 \\
(\mathrm{ND}-0.669)\end{array}$ & $\begin{array}{l}2 \pm 0 \\
(2-2)\end{array}$ \\
\hline NWWA-6 & 2 & $\begin{array}{l}387 \pm 31 \\
(356-418)\end{array}$ & $\begin{array}{l}915 \pm 223.5 \\
(691-1138)\end{array}$ & $\begin{array}{l}143 \pm 36.5 \\
(106-179)\end{array}$ & $\begin{array}{l}317 \pm 118 \\
(199-435)\end{array}$ & $\begin{array}{l}0 \pm 0.1 \\
(0-0.5)\end{array}$ & $\begin{array}{c}0 \pm 0 \\
(\mathrm{ND}-0.001)\end{array}$ & $\begin{array}{c}3 \pm 0.5 \\
(2-3)\end{array}$ \\
\hline Summary & 28 & $\begin{array}{l}333 \pm 12.1 \\
(240-461)\end{array}$ & $\begin{array}{l}658 \pm 86.6 \\
(114-1880)\end{array}$ & $\begin{array}{l}334 \pm 46.7 \\
(106-1031)\end{array}$ & $\begin{array}{l}825 \pm 252.5 \\
(121-7451)\end{array}$ & $\begin{array}{c}1 \pm 0.2 \\
(0-4)\end{array}$ & $\begin{array}{c}0 \pm 0 \\
(\mathrm{ND}-0.669)\end{array}$ & $\begin{array}{c}2 \pm 0.1 \\
(1-3)\end{array}$ \\
\hline
\end{tabular}


Table 3B. (Continued) Site and regional mean values for biomarker and ancillary data for LARGEMOUTH BASS collections made at all sites in U.S. waters from March 1994 through August 1997.

[N, number of fish; E2, 17ß Estradiol; 11KT, 11 ketotestosterone; VTG, vitellogenin; mm, millimeter; g, grams; pg, picograms; mg, milligram; mL, milliliter; ND, nondetected]

\begin{tabular}{|c|c|c|c|c|c|c|c|c|}
\hline $\begin{array}{l}\text { Regional site } \\
\text { code } \\
\text { (fig. 1) }\end{array}$ & $\mathbf{N}$ & $\begin{array}{l}\text { Length } \\
\text { (mm) }\end{array}$ & $\begin{array}{c}\text { Weight } \\
\text { (g) }\end{array}$ & $\underset{(p g / m L)}{E_{2}}$ & $\begin{array}{c}11 \mathrm{KT} \\
(\mathrm{pg} / \mathrm{mL})\end{array}$ & $\begin{array}{l}\text { Ratio of } \\
E_{2} \text { to KT }\end{array}$ & $\begin{array}{c}\text { VTG } \\
(\mathrm{mg} / \mathrm{mL})\end{array}$ & $\begin{array}{l}\text { Developmental } \\
\text { stage }\end{array}$ \\
\hline
\end{tabular}

\section{Southern Mid-Continent Region}

\section{Female non-reproductive}

\begin{tabular}{|c|c|c|c|c|c|c|c|c|}
\hline SMAR-3 & 10 & $\begin{array}{c}333 \pm 10.1 \\
(294-379)\end{array}$ & $\begin{array}{l}618 \pm 93.2 \\
(313-1255)\end{array}$ & $\begin{array}{l}1405 \pm 455 \\
(256-5221)\end{array}$ & $\begin{array}{c}355 \pm 176.8 \\
(55-1838)\end{array}$ & $\begin{array}{l}9 \pm 2.2 \\
(0-24.7)\end{array}$ & $\begin{array}{c}1 \pm 0.7 \\
(\mathrm{ND}-6.942)\end{array}$ & $\begin{array}{c}2 \pm 0.2 \\
(1-2)\end{array}$ \\
\hline SMAR-4 & 10 & $\begin{array}{l}419 \pm 25.9 \\
(305-540)\end{array}$ & $\begin{array}{c}1286 \pm 245.4 \\
(391-2400)\end{array}$ & $\begin{array}{c}530 \pm 71.8 \\
(235-845)\end{array}$ & $\begin{array}{l}288 \pm 26.1 \\
(144-429)\end{array}$ & $\begin{array}{l}2 \pm 0.3 \\
(1-4.1)\end{array}$ & $\begin{array}{c}0 \pm 0 \\
(\mathrm{ND}-0.197)\end{array}$ & $\begin{array}{l}2 \pm 0 \\
(2-2)\end{array}$ \\
\hline SMIL-2 & 9 & $\begin{array}{l}341 \pm 9.4 \\
(310-377)\end{array}$ & $\begin{array}{c}488 \pm 46.8 \\
(343-721)\end{array}$ & $\begin{array}{c}926 \pm 244.6 \\
(245-2434)\end{array}$ & $\begin{array}{c}779 \pm 105.4 \\
(95-1312)\end{array}$ & $\begin{array}{l}2 \pm 0.5 \\
(0-4.6)\end{array}$ & $\begin{array}{c}0 \pm 0 \\
(\mathrm{ND}-0.001)\end{array}$ & $\begin{array}{l}2 \pm 0 \\
(2-2)\end{array}$ \\
\hline SMLA-3 & 16 & $\begin{array}{c}348 \pm 17.8 \\
(231-462)\end{array}$ & $\begin{array}{l}702 \pm 110.7 \\
(140-1493)\end{array}$ & $\begin{array}{l}689 \pm 79.4 \\
(404-1591)\end{array}$ & $\begin{array}{l}480 \pm 53 \\
(210-899)\end{array}$ & $\begin{array}{l}2 \pm 0.2 \\
(0-3.8)\end{array}$ & $\begin{array}{c}0 \pm 0 \\
(\mathrm{ND}-0.001)\end{array}$ & $\begin{array}{c}1 \pm 0.2 \\
(0-2)\end{array}$ \\
\hline SMOK-1 & 7 & $\begin{array}{l}411 \pm 20.9 \\
(332-495)\end{array}$ & $\begin{array}{c}1161 \pm 179.6 \\
(500-1950)\end{array}$ & $\begin{array}{c}1726 \pm 368.1 \\
(705-3386)\end{array}$ & $\begin{array}{l}282 \pm 60.4 \\
(114-513)\end{array}$ & $\begin{array}{c}7 \pm 1 \\
(4-11.3)\end{array}$ & $\begin{array}{c}2 \pm 0.8 \\
(\mathrm{ND}-5.443)\end{array}$ & $\begin{array}{c}3 \pm 0.1 \\
(2-3)\end{array}$ \\
\hline SMOK-2 & 12 & $\begin{array}{c}419 \pm 15.2 \\
(351-510)\end{array}$ & $\begin{array}{c}1110 \pm 132.3 \\
(600-1900)\end{array}$ & $\begin{array}{c}970 \pm 147.2 \\
(462-2417)\end{array}$ & $\begin{array}{c}925 \pm 144.6 \\
(87-1999)\end{array}$ & $\begin{array}{c}2 \pm 0.5 \\
(0-6)\end{array}$ & $\begin{array}{c}3 \pm 1.3 \\
(\mathrm{ND}-15.897)\end{array}$ & $\begin{array}{c}3 \pm 0.1 \\
(2-3)\end{array}$ \\
\hline SMOK-3 & 9 & $\begin{array}{c}393 \pm 29.3 \\
(280-510)\end{array}$ & $\begin{array}{c}1228 \pm 255.1 \\
(350-2400)\end{array}$ & $\begin{array}{c}1491 \pm 204.8 \\
(403-2356)\end{array}$ & $\begin{array}{c}675 \pm 219.3 \\
(151-2203)\end{array}$ & $\begin{array}{l}4 \pm 0.9 \\
(0-8.6)\end{array}$ & $\begin{array}{c}1 \pm 0.3 \\
(\mathrm{ND}-3.169)\end{array}$ & $\begin{array}{c}3 \pm 0.2 \\
(2-3)\end{array}$ \\
\hline SMTN-1 & 4 & $\begin{array}{c}311 \pm 32.9 \\
(250-400)\end{array}$ & $\begin{array}{c}426 \pm 149.5 \\
(191-859)\end{array}$ & $\begin{array}{c}4283 \pm 1408.4 \\
(121-6330)\end{array}$ & $\begin{array}{c}1050 \pm 303.5 \\
(228-1512)\end{array}$ & $\begin{array}{l}9 \pm 6.3 \\
(0-27.8)\end{array}$ & $\begin{array}{c}52 \pm 18 \\
(\mathrm{ND}-77.918)\end{array}$ & $\begin{array}{c}3 \pm 0.3 \\
(2-3)\end{array}$ \\
\hline SMTN-2 & 7 & $\begin{array}{c}348 \pm 32.6 \\
(231-469)\end{array}$ & $\begin{array}{c}763 \pm 249.4 \\
(151-1822)\end{array}$ & $\begin{array}{c}374 \pm 79.5 \\
(196-690)\end{array}$ & $\begin{array}{c}183 \pm 49.8 \\
(44-422)\end{array}$ & $\begin{array}{l}3 \pm 0.8 \\
(1-5.4)\end{array}$ & $\begin{array}{c}0 \pm 0 \\
(\mathrm{ND}-0.341)\end{array}$ & $\begin{array}{l}2 \pm 0 \\
(2-2)\end{array}$ \\
\hline SMTN-3 & 11 & $\begin{array}{l}421 \pm 23.5 \\
(285-543)\end{array}$ & $\begin{array}{c}1196 \pm 185.4 \\
(340-2381)\end{array}$ & $\begin{array}{c}386 \pm 54.9 \\
(189-779)\end{array}$ & $\begin{array}{c}164 \pm 23.9 \\
(58-314)\end{array}$ & $\begin{array}{l}3 \pm 1.1 \\
(1-13.4)\end{array}$ & $\begin{array}{c}0 \pm 0 \\
(0.005-0.415)\end{array}$ & $\begin{array}{c}1 \pm 0.4 \\
(0-3)\end{array}$ \\
\hline SMTN-4 & 4 & $\begin{array}{c}286 \pm 22.4 \\
(231-325)\end{array}$ & $\begin{array}{c}295 \pm 65.2 \\
(142-425)\end{array}$ & $\begin{array}{l}362 \pm 71.1 \\
(230-563)\end{array}$ & $\begin{array}{c}65 \pm 9.2 \\
(49-89)\end{array}$ & $\begin{array}{l}6 \pm 1 \\
(4-8)\end{array}$ & $\begin{array}{c}0 \pm 0.2 \\
(\mathrm{ND}-0.925)\end{array}$ & $\begin{array}{c}3 \pm 0.3 \\
(2-3)\end{array}$ \\
\hline SMTN-5 & 10 & $\begin{array}{c}375 \pm 31.3 \\
(260-573)\end{array}$ & $\begin{array}{c}963 \pm 256.8 \\
(232-2920)\end{array}$ & $\begin{array}{c}657 \pm 240.2 \\
(52-2261)\end{array}$ & $\begin{array}{l}248 \pm 80 \\
(70-869)\end{array}$ & $\begin{array}{l}3 \pm 0.8 \\
(0-7.6)\end{array}$ & $\begin{array}{c}0 \pm 0.1 \\
(\mathrm{ND}-0.863)\end{array}$ & $\begin{array}{c}2 \pm 0.3 \\
(1-3)\end{array}$ \\
\hline SMTX-1 & 6 & $\begin{array}{c}339 \pm 32.5 \\
(306-371)\end{array}$ & $\begin{array}{c}308 \pm 3 \\
(305-311)\end{array}$ & $\begin{array}{l}313 \pm 59.9 \\
(154-566)\end{array}$ & $\begin{array}{l}178 \pm 30.4 \\
(113-314)\end{array}$ & $\begin{array}{l}2 \pm 0.4 \\
(1-3.9)\end{array}$ & $\begin{array}{c}0 \pm 0 \\
(\mathrm{ND}-0.001)\end{array}$ & $\begin{array}{c}0 \pm 0.2 \\
(0-1)\end{array}$ \\
\hline SMTX-5 & 6 & & $<$ & - Not available- & & & $\begin{array}{c}0 \pm 0 \\
(\mathrm{ND}-0.001)\end{array}$ & $\begin{array}{c}1 \pm 0.2 \\
(0-1)\end{array}$ \\
\hline SMTX-6 & 1 & & & - Not available - & & & $\begin{array}{c}0 \pm 0 \\
(\mathrm{ND}-0.001)\end{array}$ & $\begin{array}{l}0 \pm 0 \\
(0-0)\end{array}$ \\
\hline SMTX-7 & 13 & $\begin{array}{c}403 \pm 19.8 \\
(309-540)\end{array}$ & $\begin{array}{c}961 \pm 136.1 \\
(366-2100)\end{array}$ & $\begin{array}{l}721 \pm 68.3 \\
(391-1061)\end{array}$ & $\begin{array}{c}223 \pm 52.2 \\
(57-648)\end{array}$ & $\begin{array}{l}6 \pm 1.2 \\
(1-16.1)\end{array}$ & $\begin{array}{c}1 \pm 0.4 \\
(\mathrm{ND}-4.874)\end{array}$ & $\begin{array}{c}2 \pm 0.1 \\
(2-3)\end{array}$ \\
\hline Summary & 135 & $\begin{array}{l}377 \pm 6.9 \\
(231-573)\end{array}$ & $\begin{array}{l}897 \pm 53.6 \\
(140-2920)\end{array}$ & $\begin{array}{l}899 \pm 86.9 \\
(52-6330)\end{array}$ & $\begin{array}{c}416 \pm 35.5 \\
(44-2203)\end{array}$ & $\begin{array}{l}4 \pm 0.4 \\
(0-27.8)\end{array}$ & $\begin{array}{c}2 \pm 0.9 \\
(\mathrm{ND}-77.918)\end{array}$ & $\begin{array}{c}2 \pm 0.1 \\
(0-3)\end{array}$ \\
\hline
\end{tabular}


Table 3B. (Continued) Site and regional mean values for biomarker and ancillary data for LARGEMOUTH BASS collections made at all sites in U.S. waters from March 1994 through August 1997.

[N, number of fish; E2, 17ß Estradiol; 11KT, 11 ketotestosterone; VTG, vitellogenin; mm, millimeter; g, grams; pg, picograms; mg, milligram; mL, milliliter; ND, nondetected]

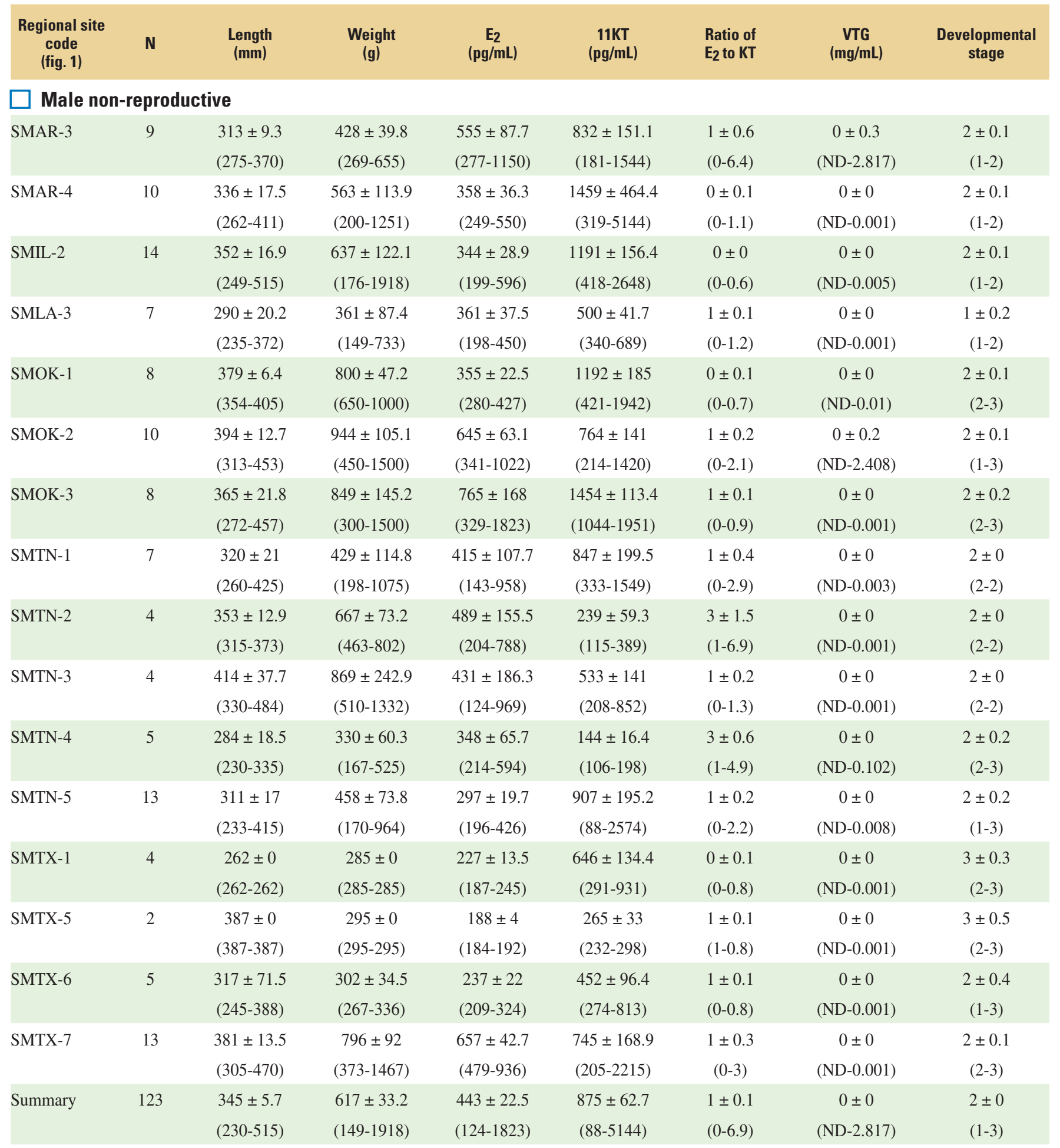


Table 3B. (Continued) Site and regional mean values for biomarker and ancillary data for LARGEMOUTH BASS collections made at all sites in U.S. waters from March 1994 through August 1997.

[N, number of fish; E2, 17ß Estradiol; 11KT, 11 ketotestosterone; VTG, vitellogenin; mm, millimeter; g, grams; pg, picograms; mg, milligram; mL, milliliter; ND, nondetected]

\begin{tabular}{|c|c|c|c|c|c|c|c|c|}
\hline $\begin{array}{c}\text { Regional site } \\
\text { code } \\
\text { (fig. 1) }\end{array}$ & $\mathbf{N}$ & $\begin{array}{c}\text { Length } \\
\text { (mm) }\end{array}$ & $\begin{array}{l}\text { Weight } \\
\text { (g) }\end{array}$ & $\underset{(\mathrm{pg} / \mathrm{mL})}{\mathrm{E}_{2}}$ & $\begin{array}{c}11 \mathrm{KT} \\
(\mathrm{pg} / \mathrm{mL})\end{array}$ & $\begin{array}{l}\text { Ratio of } \\
E_{2} \text { to KT }\end{array}$ & $\underset{(\mathrm{mg} / \mathrm{mL})}{\text { VTG }}$ & $\begin{array}{l}\text { Developmental } \\
\text { stage }\end{array}$ \\
\hline \multicolumn{9}{|c|}{$\square$ Female non-reproductive } \\
\hline SWCA-1 & 23 & $\begin{array}{c}398 \pm 17.3 \\
(273-580)\end{array}$ & $\begin{array}{c}1068 \pm 147.6 \\
(222-2804)\end{array}$ & $\begin{array}{l}802 \pm 77.1 \\
(289-1539)\end{array}$ & $\begin{array}{c}221 \pm 27.9 \\
(38-662)\end{array}$ & $\begin{array}{c}5 \pm 0.9 \\
(1-20.8)\end{array}$ & $\begin{array}{c}0 \pm 0 \\
(\mathrm{ND}-0.564)\end{array}$ & $\begin{array}{c}1 \pm 0.1 \\
(0-1)\end{array}$ \\
\hline SWCA-2 & 19 & $\begin{array}{l}392 \pm 17.3 \\
(285-595)\end{array}$ & $\begin{array}{c}1046 \pm 116.2 \\
(320-2020)\end{array}$ & $\begin{array}{c}455 \pm 130.7 \\
(54-2229)\end{array}$ & $\begin{array}{c}101 \pm 22.5 \\
(12-362)\end{array}$ & $\begin{array}{c}5 \pm 1 \\
(0-17.4)\end{array}$ & $\begin{array}{c}0 \pm 0.1 \\
(\mathrm{ND}-1.123)\end{array}$ & $\begin{array}{c}1 \pm 0.1 \\
(1-2)\end{array}$ \\
\hline SWCA-3 & 31 & $\begin{array}{l}376 \pm 10.5 \\
(270-482)\end{array}$ & $\begin{array}{l}912 \pm 80.8 \\
(277-2010)\end{array}$ & $\begin{array}{l}374 \pm 60.8 \\
(29-1345)\end{array}$ & $\begin{array}{c}273 \pm 36.6 \\
(59-811)\end{array}$ & $\begin{array}{l}2 \pm 0.3 \\
(0-9.3)\end{array}$ & $\begin{array}{c}0 \pm 0.1 \\
(\mathrm{ND}-3.541)\end{array}$ & $\begin{array}{c}1 \pm 0.1 \\
(1-2)\end{array}$ \\
\hline SWCA-1 & 11 & $\begin{array}{l}316 \pm 20.1 \\
(252-448)\end{array}$ & $\begin{array}{c}514 \pm 117.6 \\
(197-1389)\end{array}$ & $\begin{array}{c}789 \pm 119.4 \\
(207-1370)\end{array}$ & $\begin{array}{c}904 \pm 194.9 \\
(98-1894)\end{array}$ & $\begin{array}{l}1 \pm 0.3 \\
(0-3.4)\end{array}$ & $\begin{array}{c}0 \pm 0.2 \\
(\mathrm{ND}-1.8)\end{array}$ & $\begin{array}{l}3 \pm 0 \\
(3-3)\end{array}$ \\
\hline SWCA-2 & 21 & $\begin{array}{l}351 \pm 9.4 \\
(258-435)\end{array}$ & $\begin{array}{l}709 \pm 57.1 \\
(207-1333)\end{array}$ & $\begin{array}{c}187 \pm 51.1 \\
(19-821)\end{array}$ & $\begin{array}{l}202 \pm 72 \\
(18-1131)\end{array}$ & $\begin{array}{l}2 \pm 0.3 \\
(0-4.2)\end{array}$ & $\begin{array}{c}0 \pm 0 \\
(\mathrm{ND}-0.039)\end{array}$ & $\begin{array}{l}3 \pm 0 \\
(2-3)\end{array}$ \\
\hline SWCA-3 & 20 & $\begin{array}{l}354 \pm 12.4 \\
(226-475)\end{array}$ & $\begin{array}{l}677 \pm 56.4 \\
(230-1263)\end{array}$ & $\begin{array}{l}197 \pm 39 \\
(35-761)\end{array}$ & $\begin{array}{c}260 \pm 37.9 \\
(97-656)\end{array}$ & $\begin{array}{c}1 \pm 0.2 \\
(0-4)\end{array}$ & $\begin{array}{c}0 \pm 0 \\
(\mathrm{ND}-0.117)\end{array}$ & $\begin{array}{l}3 \pm 0 \\
(3-3)\end{array}$ \\
\hline Summary & 52 & $\begin{array}{l}345 \pm 7.6 \\
(226-475)\end{array}$ & $\begin{array}{l}656 \pm 40.7 \\
(197-1389)\end{array}$ & $\begin{array}{c}318 \pm 48.9 \\
(19-1370)\end{array}$ & $\begin{array}{c}373 \pm 64 \\
(18-1894)\end{array}$ & $\begin{array}{l}1 \pm 0.1 \\
(0-4.2)\end{array}$ & $\begin{array}{c}0 \pm 0 \\
(\mathrm{ND}-1.8)\end{array}$ & $\begin{array}{l}3 \pm 0 \\
(2-3)\end{array}$ \\
\hline
\end{tabular}


Table 4. All individual COMMON CARP values for biomarker and ancillary data from collections made at sites in U.S. waters from August 1994 through October 1997.

[Region: NE, northeast; NW, northwest; SE, southeast; SW, southwest; NMC, northern mid-continent; SMC, southern mid-continent. Season: NR, non-reproductive. R, reproductive. Sex: F, female, M, male. E2, 17 ß Estradiol; 11KT, 11 ketotestosterone; VTG, vitellogenin; mm, millimeter; g, grams; pg, picograms; mL, milliliter; mg, milligram; ND, not detected; NA, not available]

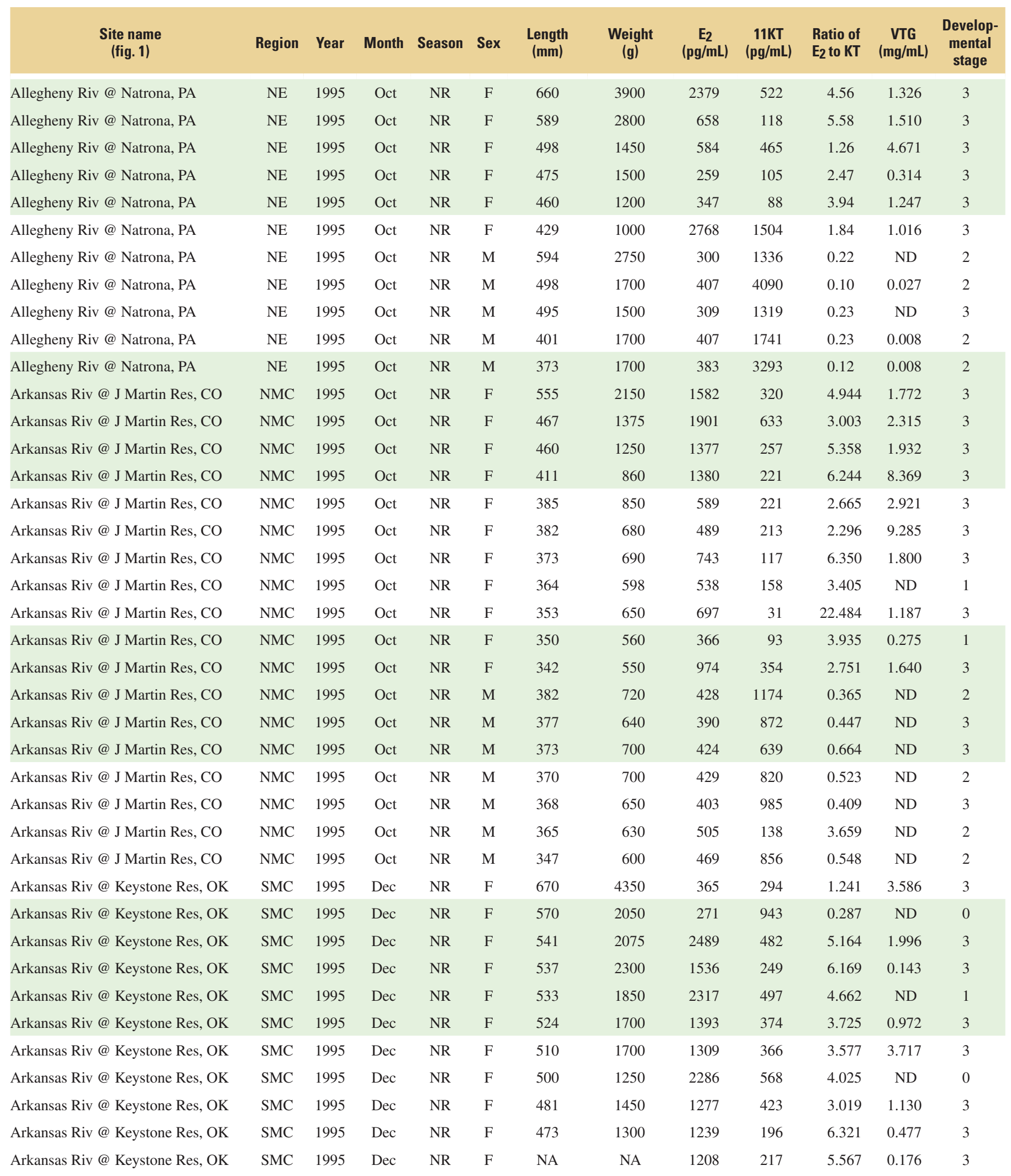


Table 4. (Continued) All individual COMMON CARP values for biomarker and ancillary data from collections made at sites in U.S. waters from August 1994 through October 1997.

[Region: NE, northeast; NW, northwest; SE, southeast; SW, southwest; NMC, northern mid-continent; SMC, southern mid-continent.

Season: NR, non-reproductive. R, reproductive. Sex: F, female, M, male. E2, 17 ß Estradiol; 11KT, 11 ketotestosterone; VTG, vitellogenin; mm, millimeter; g, grams; pg, picograms; mL, milliliter; mg, milligram; ND, not detected; NA, not available]

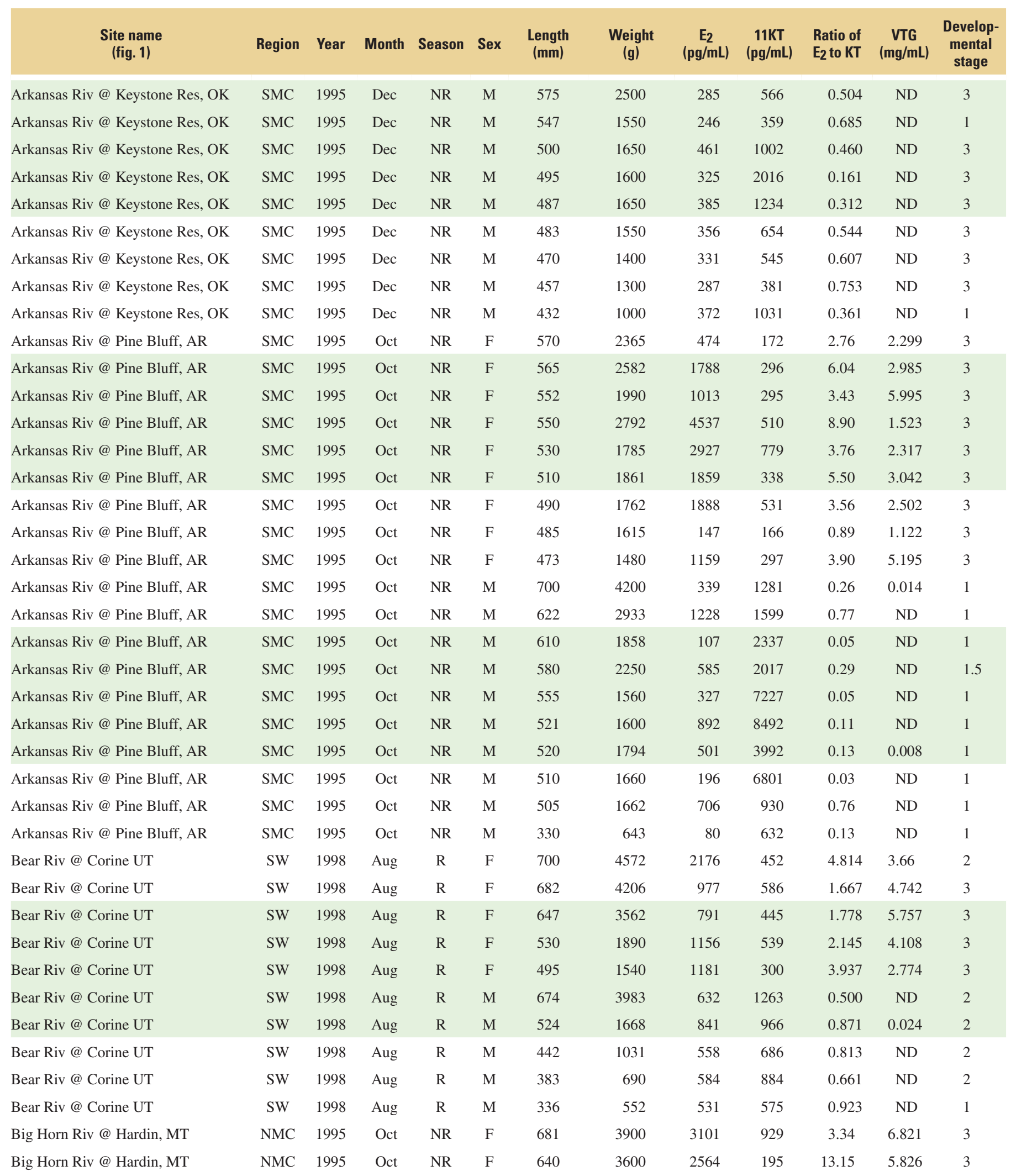


Table 4. (Continued) All individual COMMON CARP values for biomarker and ancillary data from collections made at sites in U.S. waters from August 1994 through October 1997.

[Region: NE, northeast; NW, northwest; SE, southeast; SW, southwest; NMC, northern mid-continent; SMC, southern mid-continent.

Season: NR, non-reproductive. R, reproductive. Sex: F, female, M, male. E2, 17 ß Estradiol; 11KT, 11 ketotestosterone; VTG, vitellogenin; mm, millimeter; g, grams; pg, picograms; mL, milliliter; mg, milligram; ND, not detected; NA, not available]

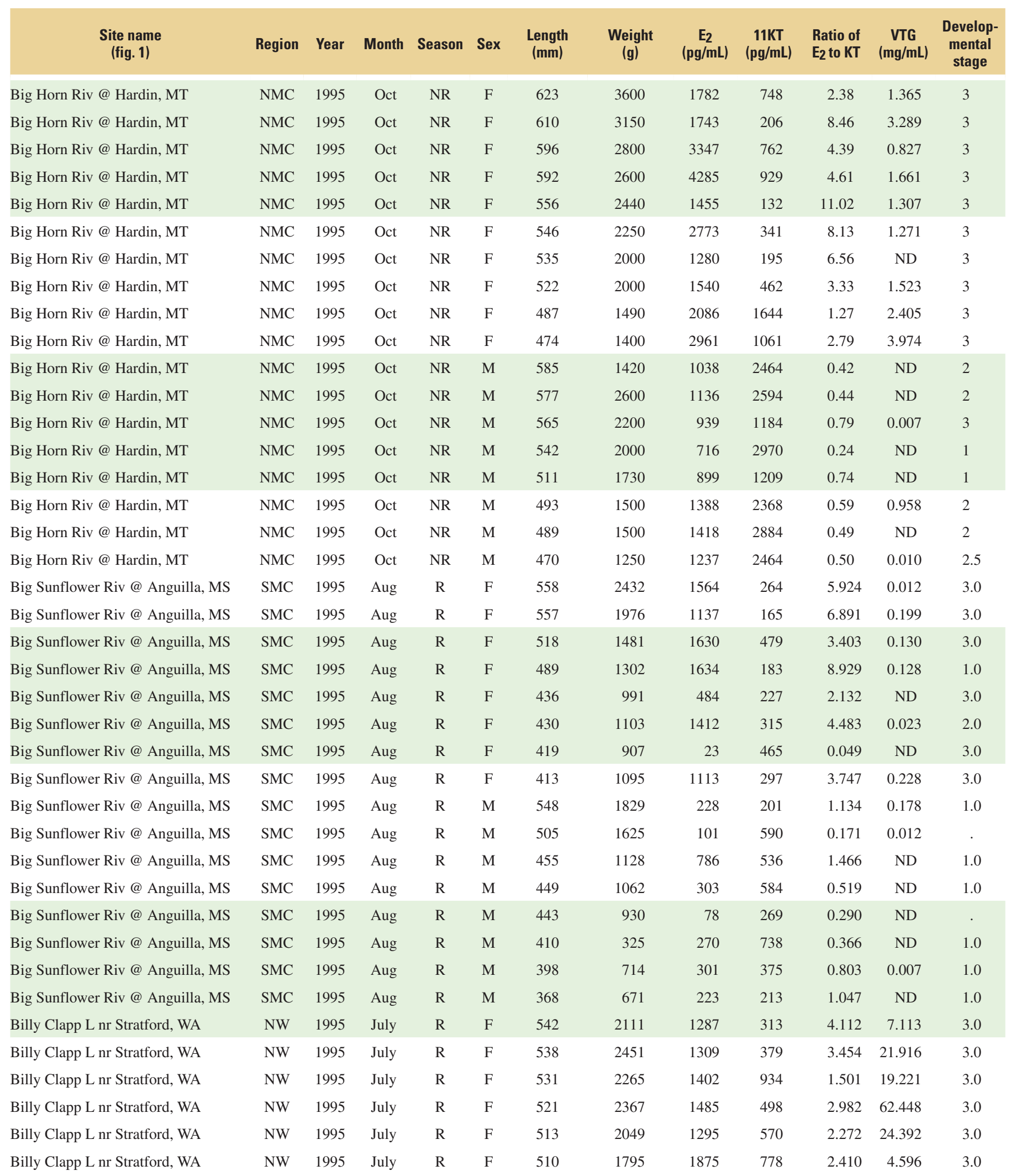


Table 4. (Continued) All individual COMMON CARP values for biomarker and ancillary data from collections made at sites in U.S. waters from August 1994 through October 1997.

[Region: NE, northeast; NW, northwest; SE, southeast; SW, southwest; NMC, northern mid-continent; SMC, southern mid-continent. Season: NR, non-reproductive. R, reproductive. Sex: F, female, M, male. E2, 17 ß Estradiol; 11KT, 11 ketotestosterone; VTG, vitellogenin; mm, millimeter; g, grams; pg, picograms; mL, milliliter; mg, milligram; ND, not detected; NA, not available]

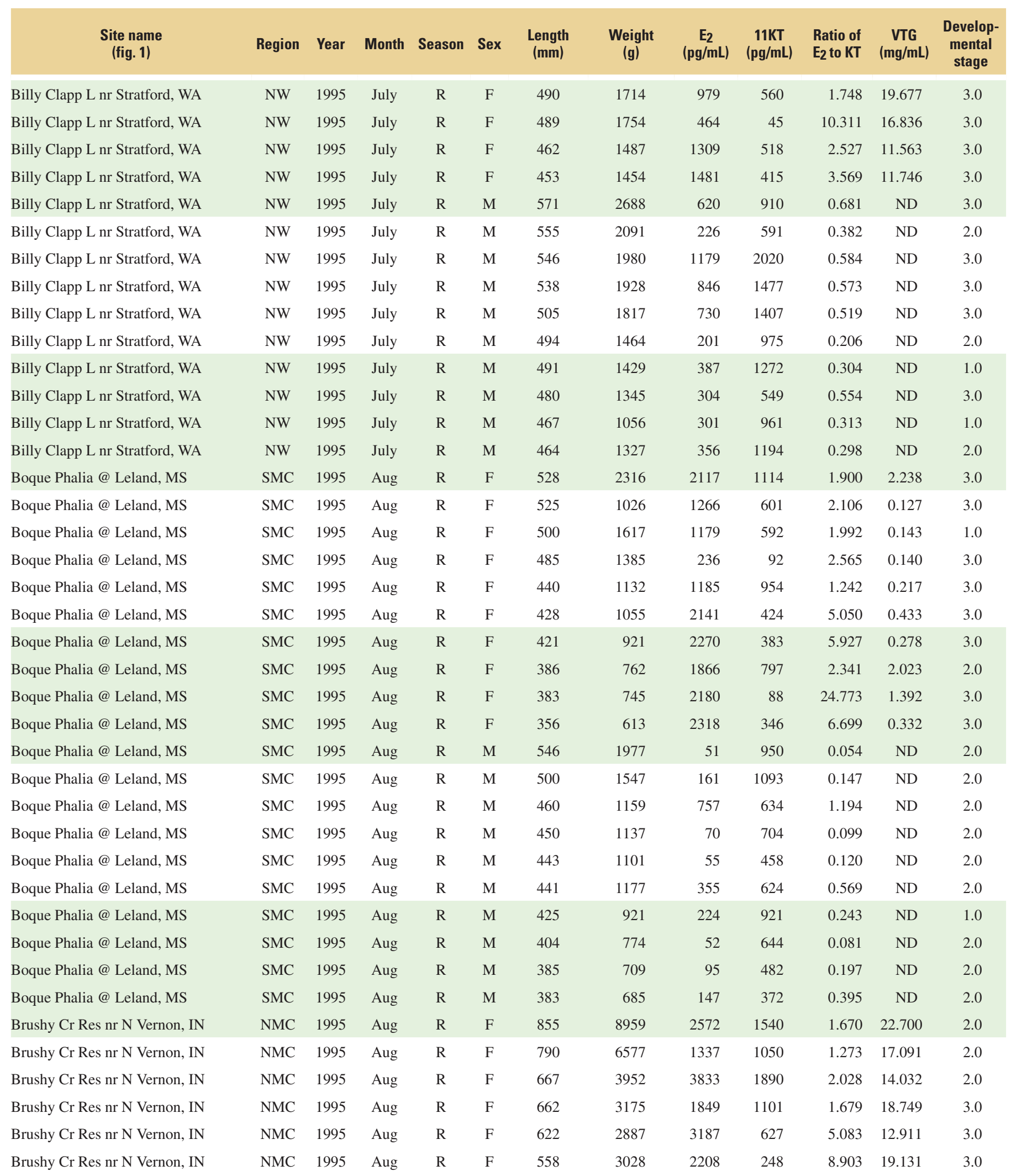


Table 4. (Continued) All individual COMMON CARP values for biomarker and ancillary data from collections made at sites in U.S. waters from August 1994 through October 1997.

[Region: NE, northeast; NW, northwest; SE, southeast; SW, southwest; NMC, northern mid-continent; SMC, southern mid-continent.

Season: NR, non-reproductive. R, reproductive. Sex: F, female, M, male. E2, 17 ß Estradiol; 11KT, 11 ketotestosterone; VTG, vitellogenin;

mm, millimeter; g, grams; pg, picograms; mL, milliliter; mg, milligram; ND, not detected; NA, not available]

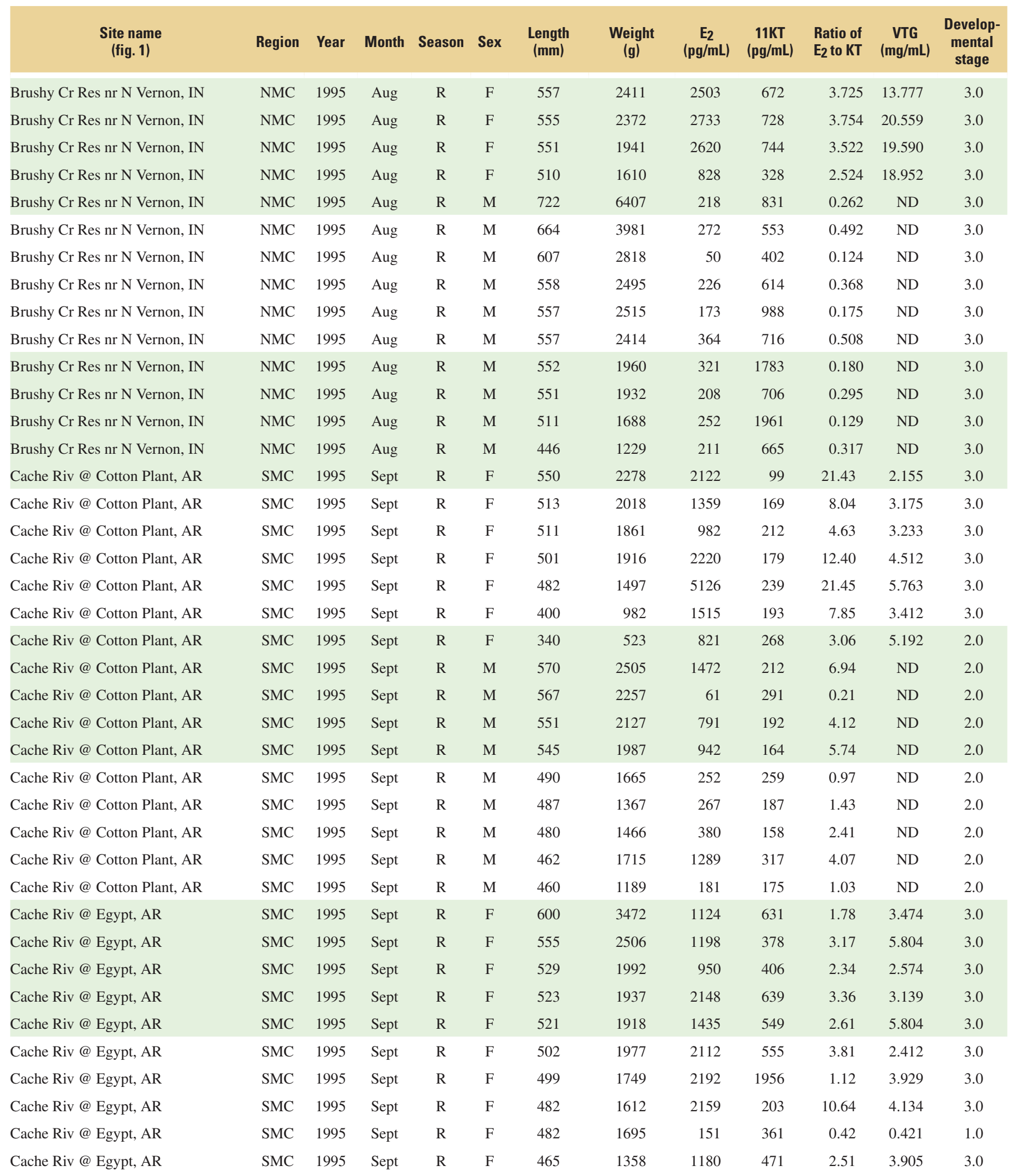


Table 4. (Continued) All individual COMMON CARP values for biomarker and ancillary data from collections made at sites in U.S. waters from August 1994 through October 1997.

[Region: NE, northeast; NW, northwest; SE, southeast; SW, southwest; NMC, northern mid-continent; SMC, southern mid-continent.

Season: NR, non-reproductive. R, reproductive. Sex: F, female, M, male. E2, 17 ß Estradiol; 11KT, 11 ketotestosterone; VTG, vitellogenin;

mm, millimeter; g, grams; pg, picograms; mL, milliliter; mg, milligram; ND, not detected; NA, not available]

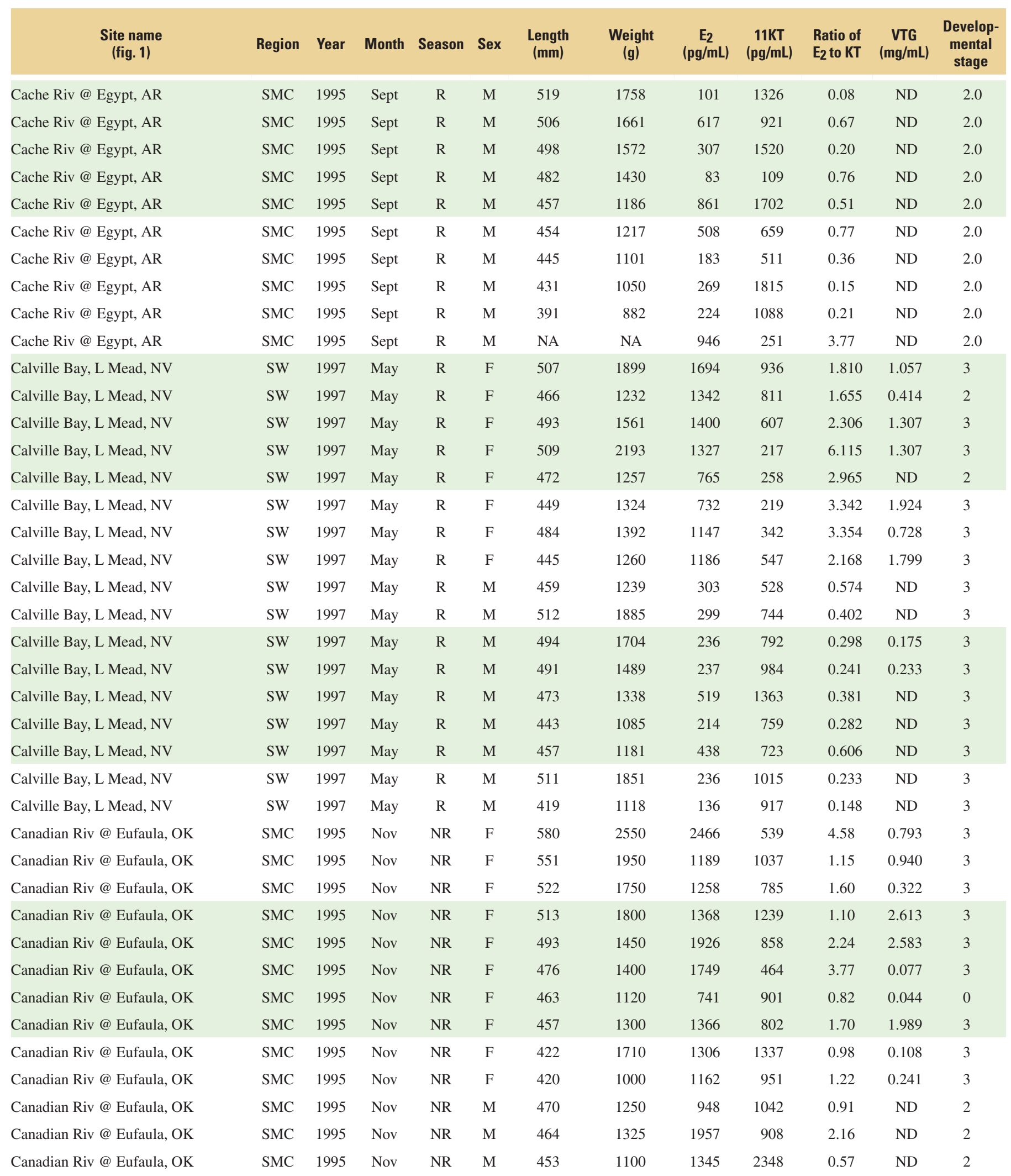


Table 4. (Continued) All individual COMMON CARP values for biomarker and ancillary data from collections made at sites in U.S. waters from August 1994 through October 1997.

[Region: NE, northeast; NW, northwest; SE, southeast; SW, southwest; NMC, northern mid-continent; SMC, southern mid-continent.

Season: NR, non-reproductive. R, reproductive. Sex: F, female, M, male. E2, 17 ß Estradiol; 11KT, 11 ketotestosterone; VTG, vitellogenin;

mm, millimeter; g, grams; pg, picograms; mL, milliliter; mg, milligram; ND, not detected; NA, not available]

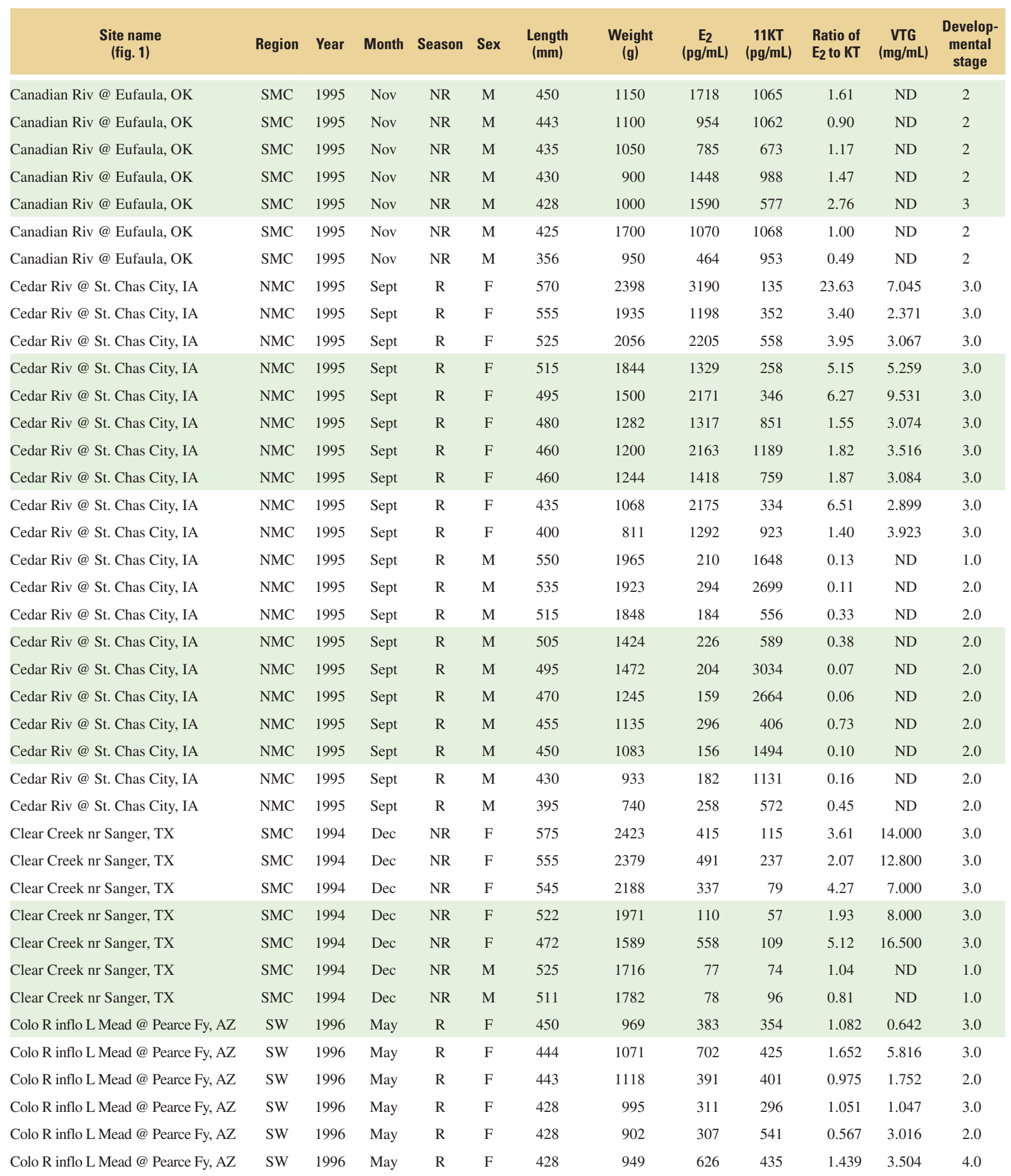


Table 4. (Continued) All individual COMMON CARP values for biomarker and ancillary data from collections made at sites in U.S. waters from August 1994 through October 1997.

[Region: NE, northeast; NW, northwest; SE, southeast; SW, southwest; NMC, northern mid-continent; SMC, southern mid-continent.

Season: NR, non-reproductive. R, reproductive. Sex: F, female, M, male. E2, 17 ß Estradiol; 11KT, 11 ketotestosterone; VTG, vitellogenin;

mm, millimeter; g, grams; pg, picograms; mL, milliliter; mg, milligram; ND, not detected; NA, not available]

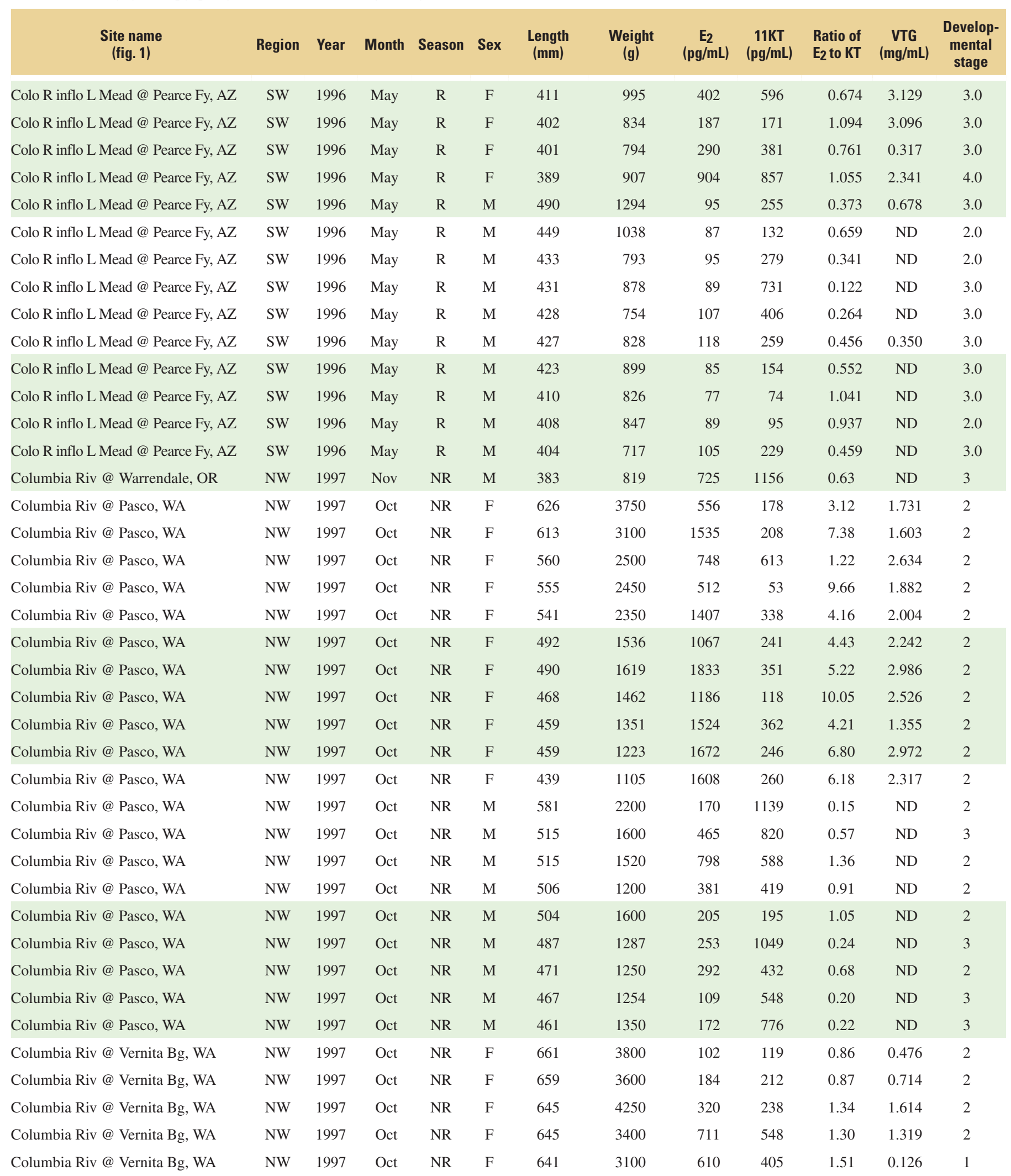


Table 4. (Continued) All individual COMMON CARP values for biomarker and ancillary data from collections made at sites in U.S. waters from August 1994 through October 1997.

[Region: NE, northeast; NW, northwest; SE, southeast; SW, southwest; NMC, northern mid-continent; SMC, southern mid-continent.

Season: NR, non-reproductive. R, reproductive. Sex: F, female, M, male. E2, 17 ß Estradiol; 11KT, 11 ketotestosterone; VTG, vitellogenin;

mm, millimeter; g, grams; pg, picograms; mL, milliliter; mg, milligram; ND, not detected; NA, not available]

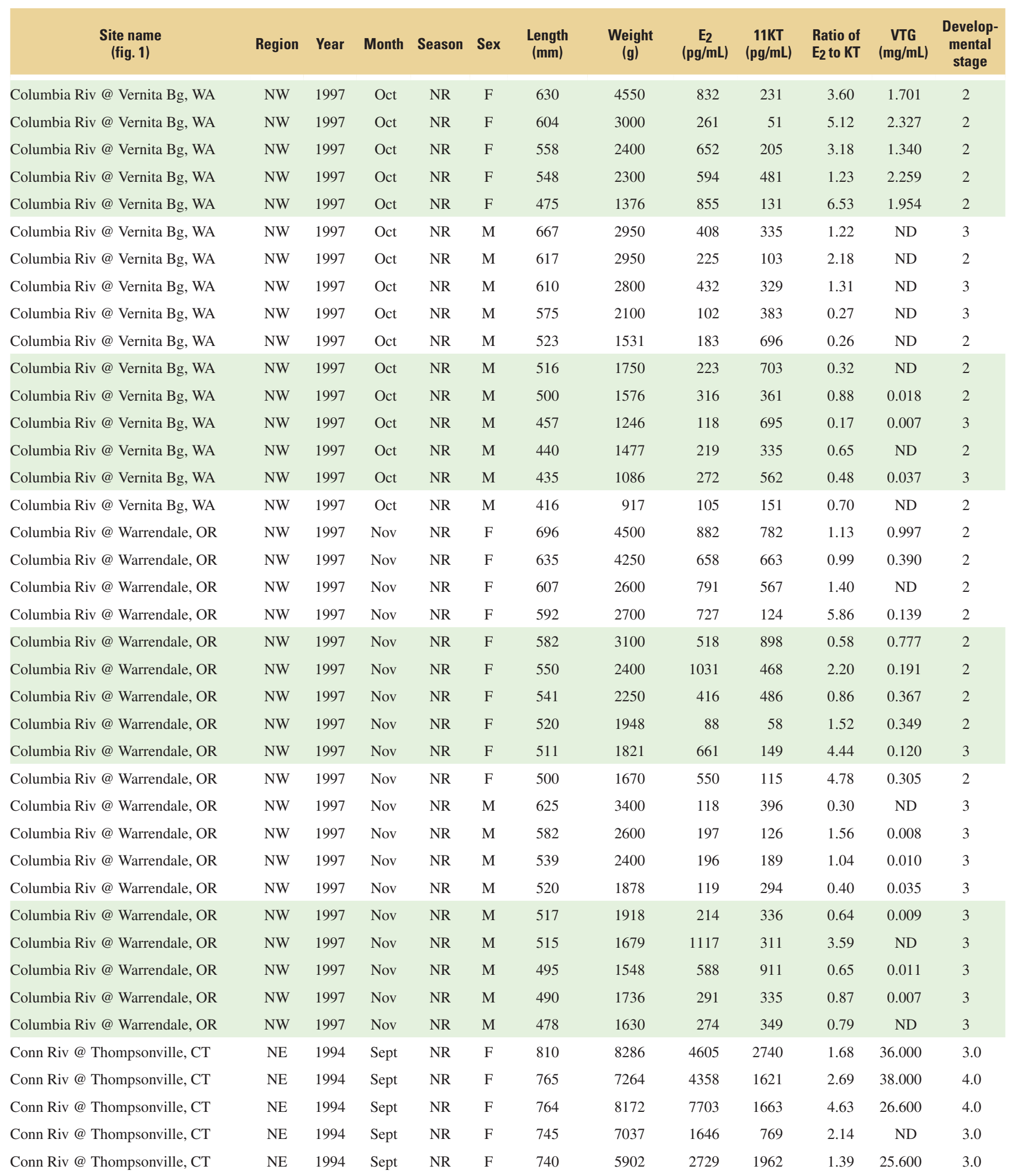


Table 4. (Continued) All individual COMMON CARP values for biomarker and ancillary data from collections made at sites in U.S. waters from August 1994 through October 1997.

[Region: NE, northeast; NW, northwest; SE, southeast; SW, southwest; NMC, northern mid-continent; SMC, southern mid-continent.

Season: NR, non-reproductive. R, reproductive. Sex: F, female, M, male. E2, 17 ß Estradiol; 11KT, 11 ketotestosterone; VTG, vitellogenin;

mm, millimeter; g, grams; pg, picograms; mL, milliliter; mg, milligram; ND, not detected; NA, not available]

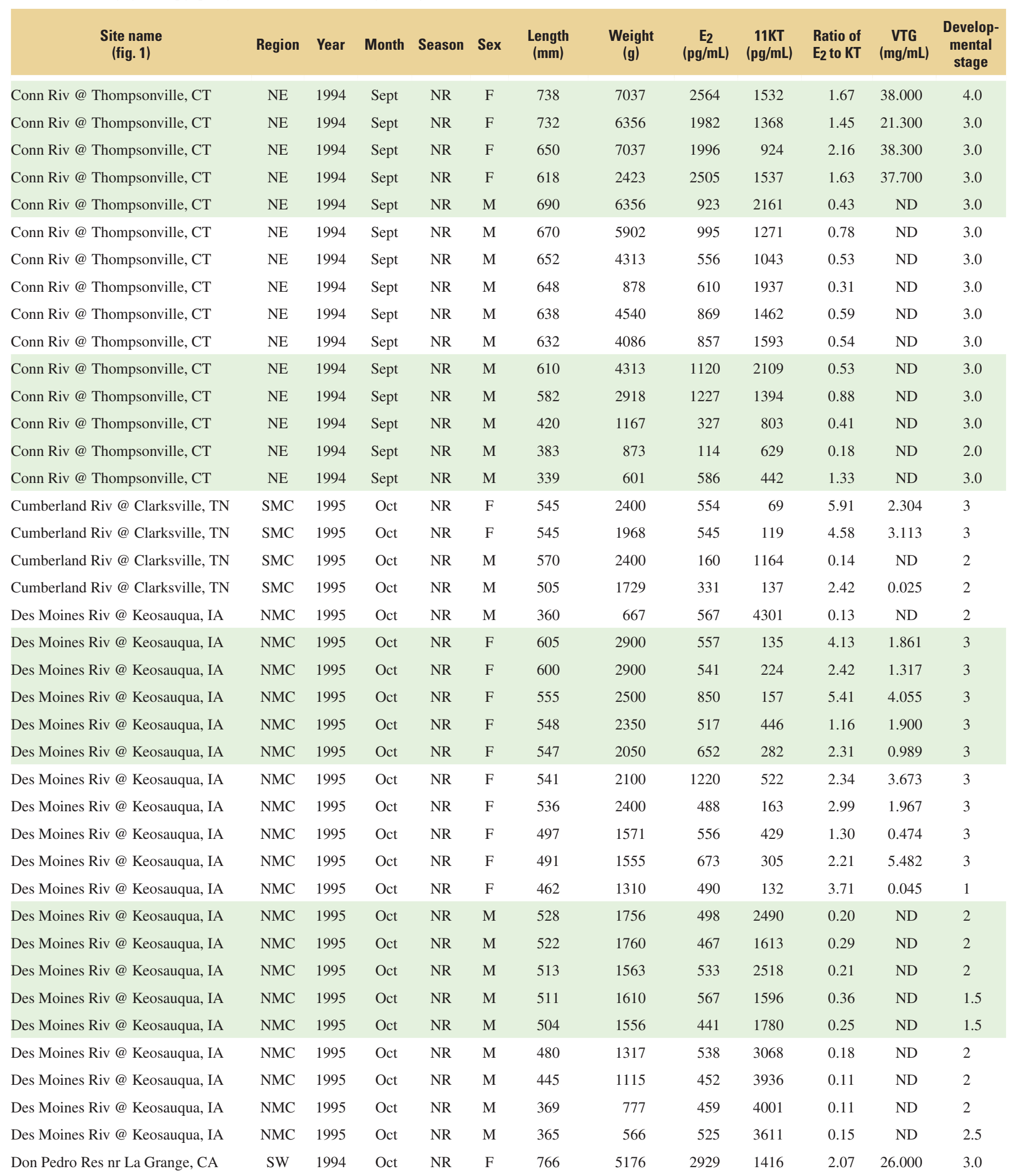


Table 4. (Continued) All individual COMMON CARP values for biomarker and ancillary data from collections made at sites in U.S. waters from August 1994 through October 1997.

[Region: NE, northeast; NW, northwest; SE, southeast; SW, southwest; NMC, northern mid-continent; SMC, southern mid-continent.

Season: NR, non-reproductive. R, reproductive. Sex: F, female, M, male. E2, 17 ß Estradiol; 11KT, 11 ketotestosterone; VTG, vitellogenin; mm, millimeter; g, grams; pg, picograms; mL, milliliter; mg, milligram; ND, not detected; NA, not available]

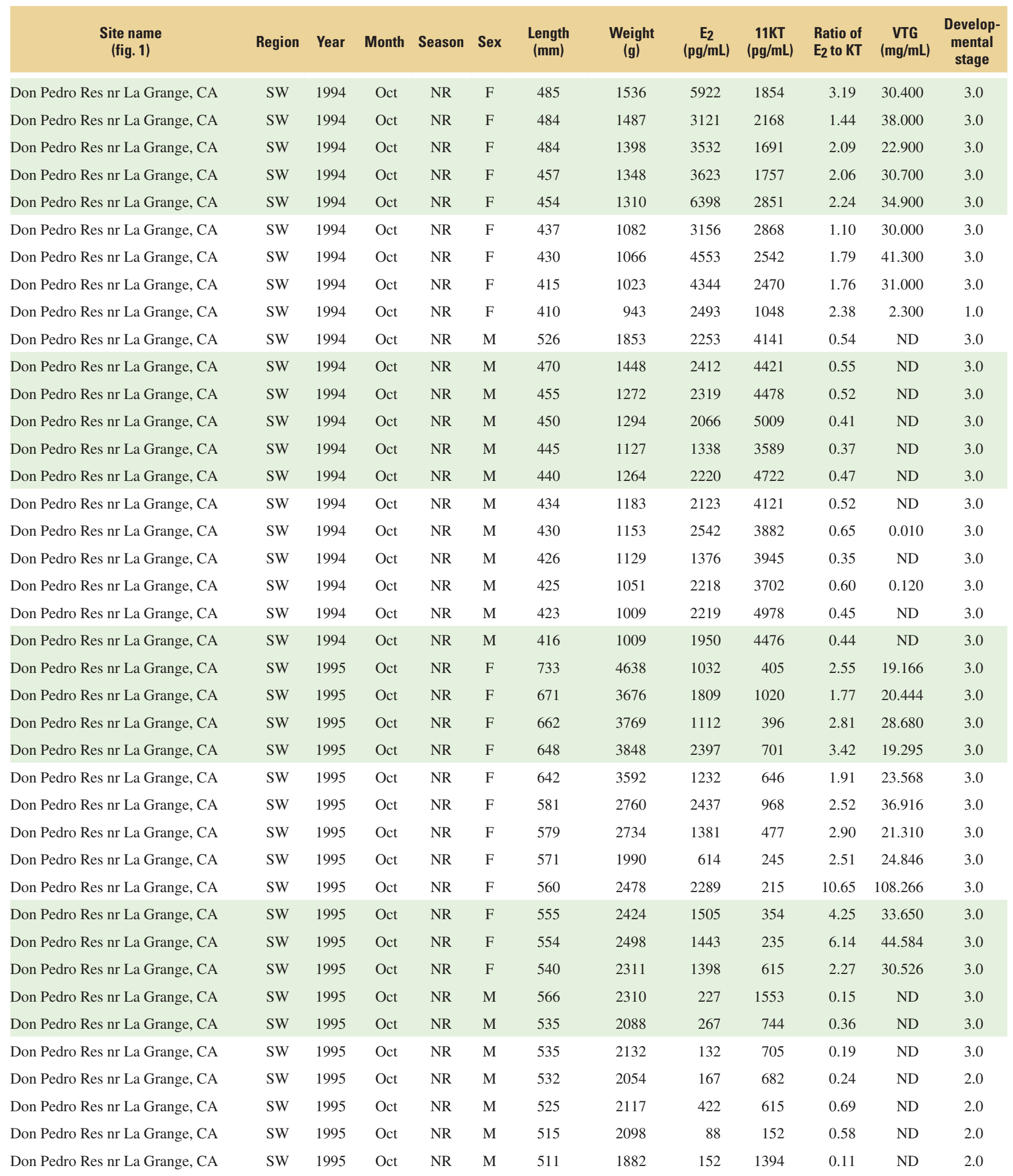


Table 4. (Continued) All individual COMMON CARP values for biomarker and ancillary data from collections made at sites in U.S. waters from August 1994 through October 1997.

[Region: NE, northeast; NW, northwest; SE, southeast; SW, southwest; NMC, northern mid-continent; SMC, southern mid-continent. Season: NR, non-reproductive. R, reproductive. Sex: F, female, M, male. E2, 17 ß Estradiol; 11KT, 11 ketotestosterone; VTG, vitellogenin; mm, millimeter; g, grams; pg, picograms; mL, milliliter; mg, milligram; ND, not detected; NA, not available]

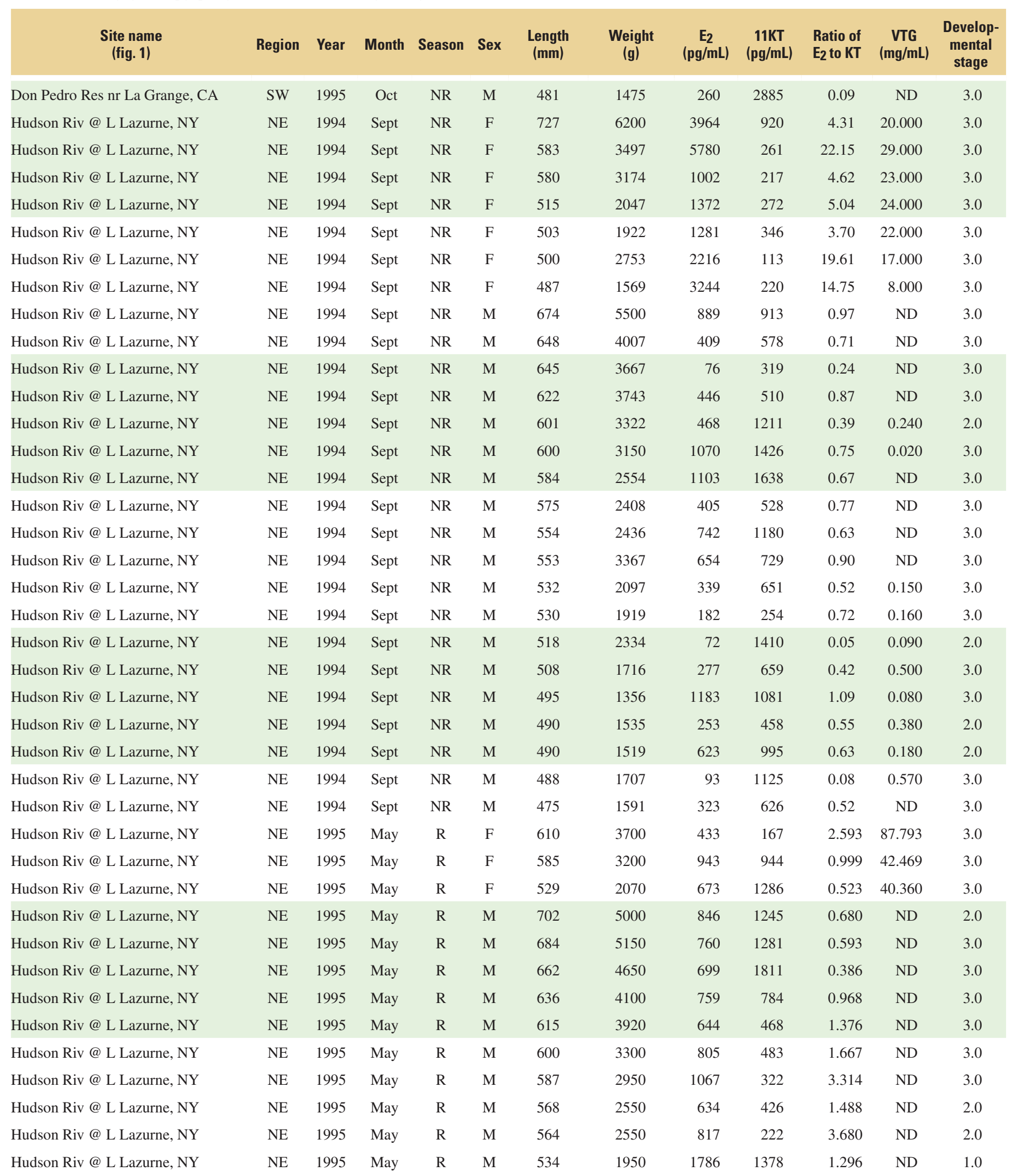


Table 4. (Continued) All individual COMMON CARP values for biomarker and ancillary data from collections made at sites in U.S. waters from August 1994 through October 1997.

[Region: NE, northeast; NW, northwest; SE, southeast; SW, southwest; NMC, northern mid-continent; SMC, southern mid-continent.

Season: NR, non-reproductive. R, reproductive. Sex: F, female, M, male. E2, 17 ß Estradiol; 11KT, 11 ketotestosterone; VTG, vitellogenin; mm, millimeter; g, grams; pg, picograms; mL, milliliter; mg, milligram; ND, not detected; NA, not available]

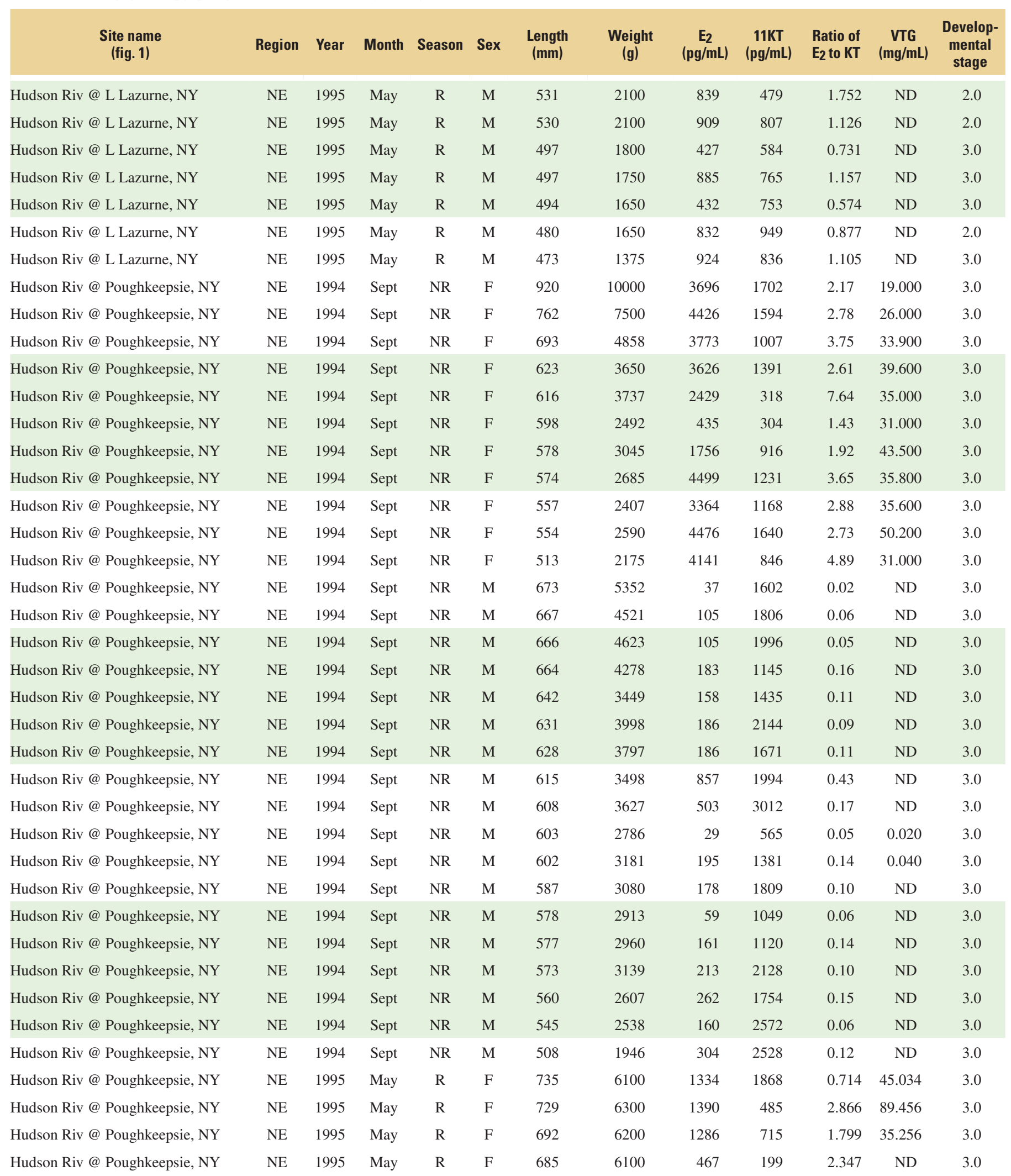


Table 4. (Continued) All individual COMMON CARP values for biomarker and ancillary data from collections made at sites in U.S. waters from August 1994 through October 1997.

[Region: NE, northeast; NW, northwest; SE, southeast; SW, southwest; NMC, northern mid-continent; SMC, southern mid-continent. Season: NR, non-reproductive. R, reproductive. Sex: F, female, M, male. E2, 17 ß Estradiol; 11KT, 11 ketotestosterone; VTG, vitellogenin; mm, millimeter; g, grams; pg, picograms; mL, milliliter; mg, milligram; ND, not detected; NA, not available]

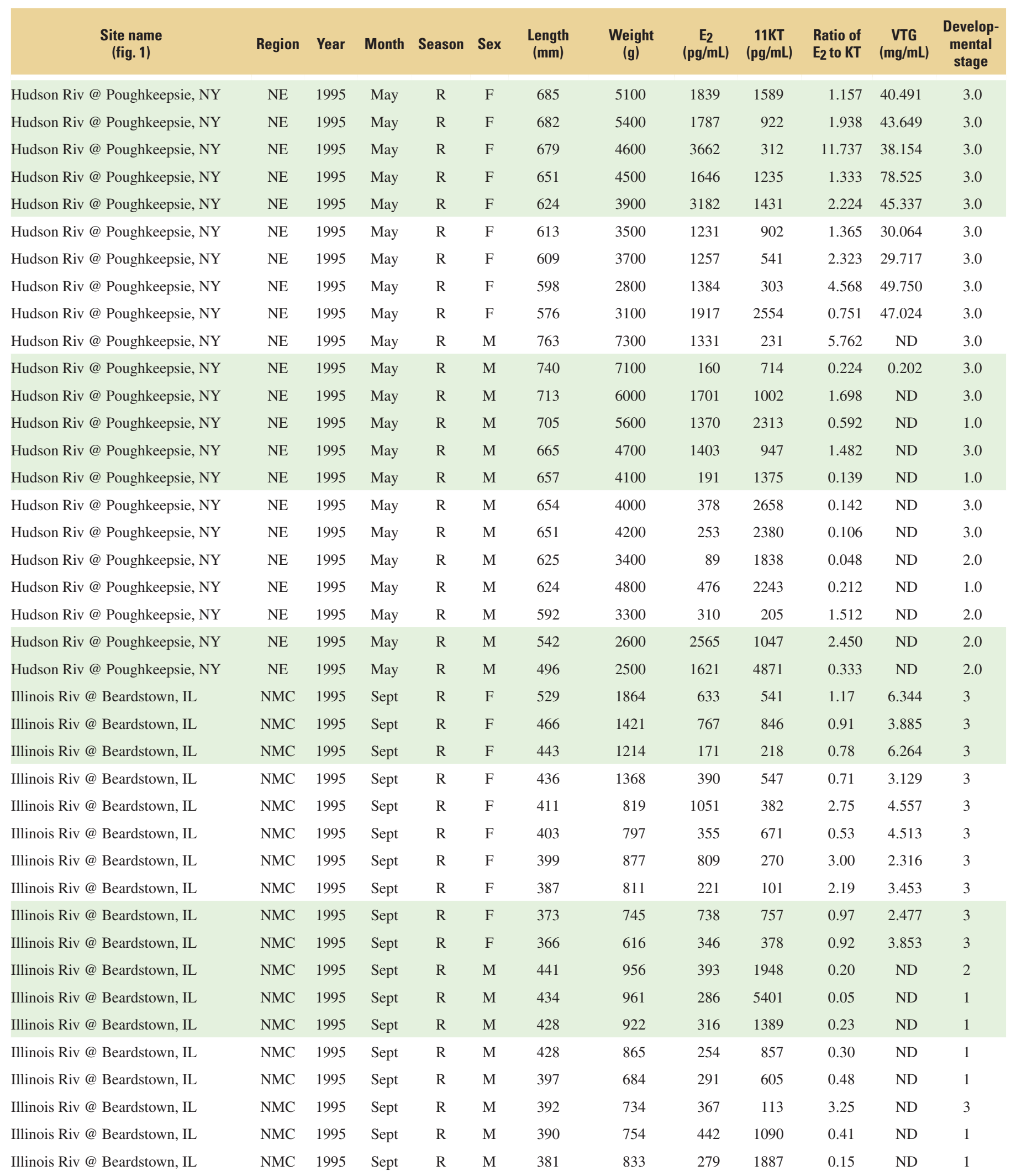


Table 4. (Continued) All individual COMMON CARP values for biomarker and ancillary data from collections made at sites in U.S. waters from August 1994 through October 1997.

[Region: NE, northeast; NW, northwest; SE, southeast; SW, southwest; NMC, northern mid-continent; SMC, southern mid-continent.

Season: NR, non-reproductive. R, reproductive. Sex: F, female, M, male. E2, 17 ß Estradiol; 11KT, 11 ketotestosterone; VTG, vitellogenin; mm, millimeter; g, grams; pg, picograms; mL, milliliter; mg, milligram; ND, not detected; NA, not available]

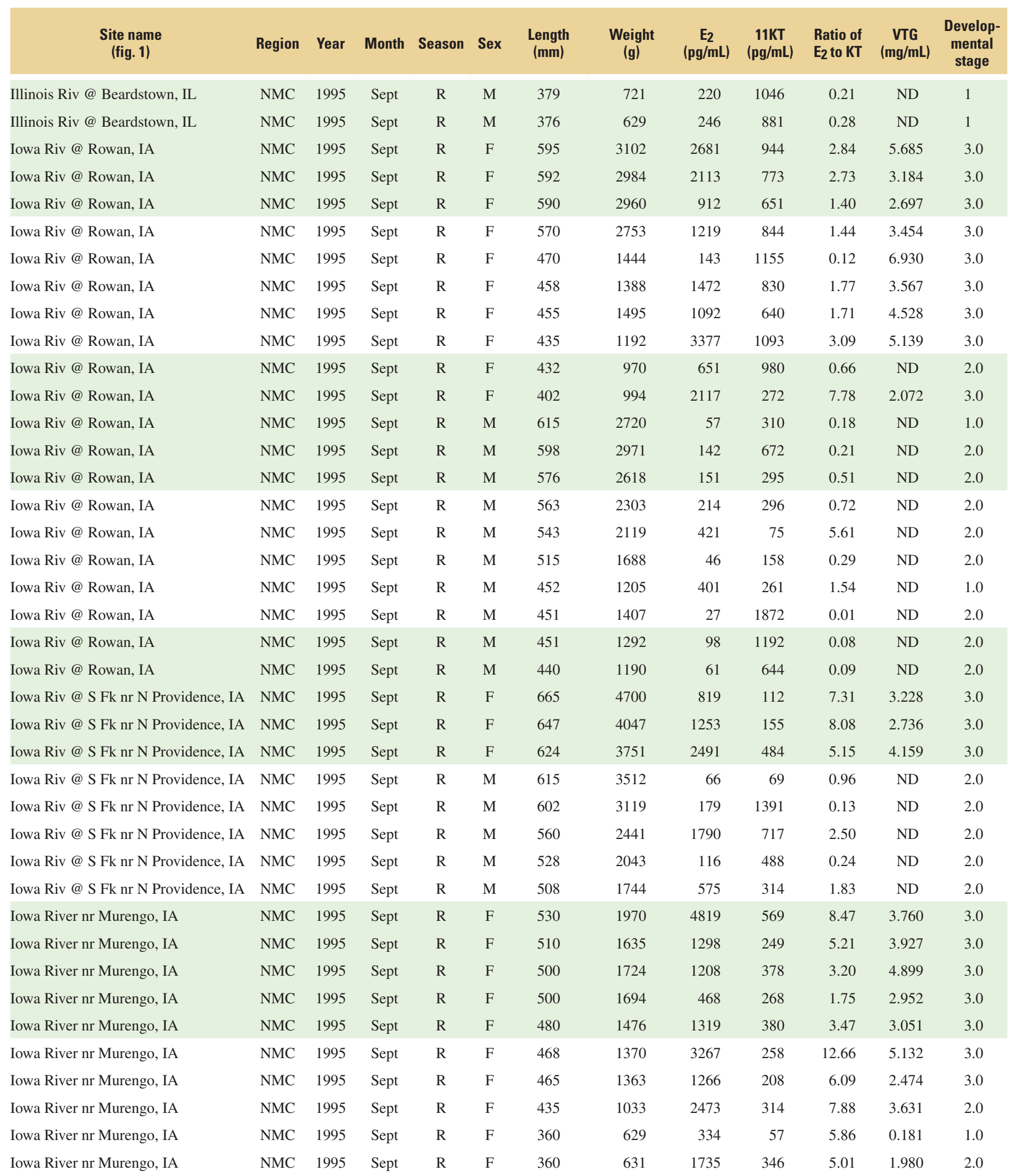


Table 4. (Continued) All individual COMMON CARP values for biomarker and ancillary data from collections made at sites in U.S. waters from August 1994 through October 1997.

[Region: NE, northeast; NW, northwest; SE, southeast; SW, southwest; NMC, northern mid-continent; SMC, southern mid-continent. Season: NR, non-reproductive. R, reproductive. Sex: F, female, M, male. E2, 17 ß Estradiol; 11KT, 11 ketotestosterone; VTG, vitellogenin; mm, millimeter; g, grams; pg, picograms; mL, milliliter; mg, milligram; ND, not detected; NA, not available]

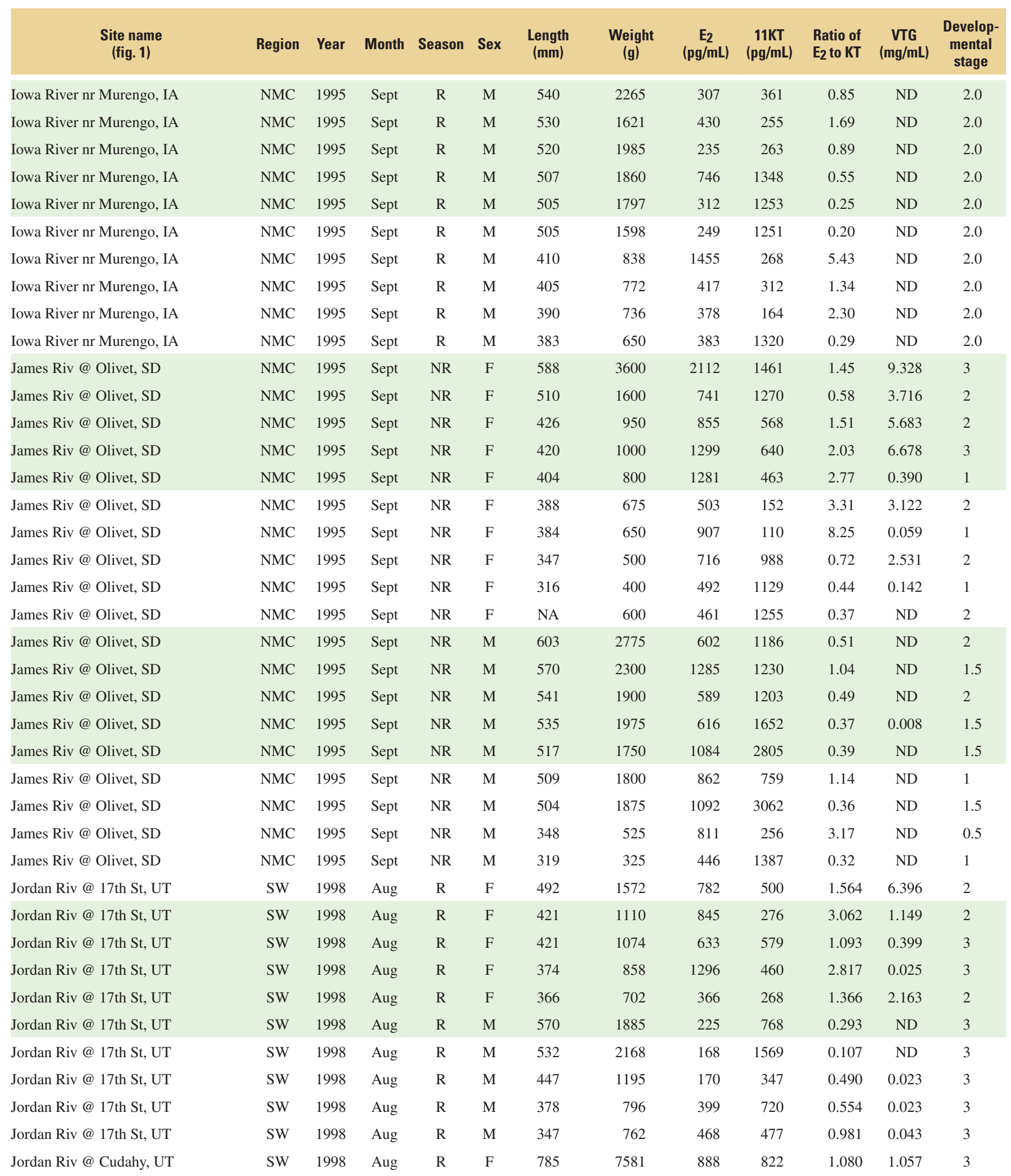


Table 4. (Continued) All individual COMMON CARP values for biomarker and ancillary data from collections made at sites in U.S. waters from August 1994 through October 1997.

[Region: NE, northeast; NW, northwest; SE, southeast; SW, southwest; NMC, northern mid-continent; SMC, southern mid-continent.

Season: NR, non-reproductive. R, reproductive. Sex: F, female, M, male. E2, 17 ß Estradiol; 11KT, 11 ketotestosterone; VTG, vitellogenin; mm, millimeter; g, grams; pg, picograms; mL, milliliter; mg, milligram; ND, not detected; NA, not available]

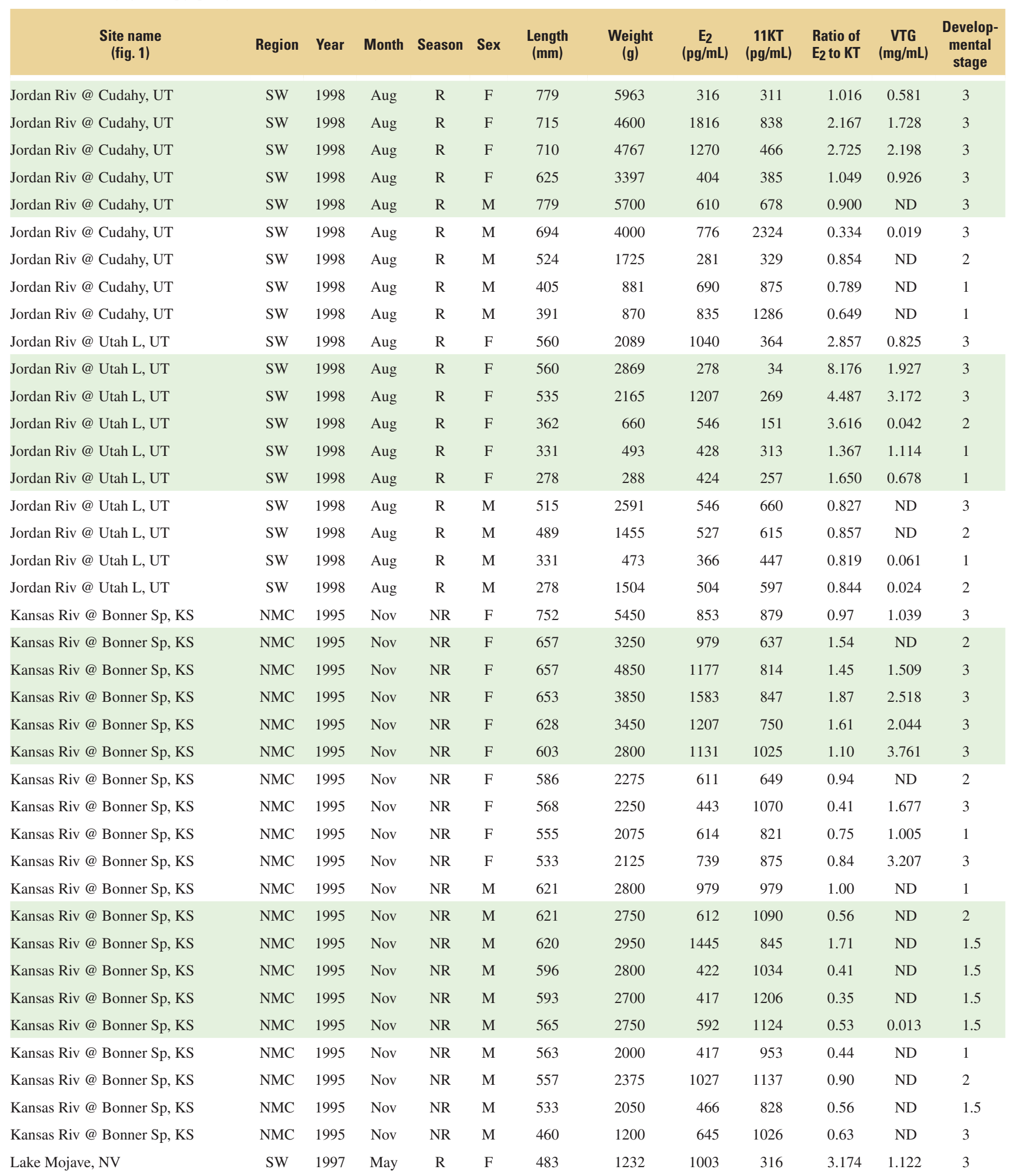


Table 4. (Continued) All individual COMMON CARP values for biomarker and ancillary data from collections made at sites in U.S. waters from August 1994 through October 1997.

[Region: NE, northeast; NW, northwest; SE, southeast; SW, southwest; NMC, northern mid-continent; SMC, southern mid-continent.

Season: NR, non-reproductive. R, reproductive. Sex: F, female, M, male. E2, 17 ß Estradiol; 11KT, 11 ketotestosterone; VTG, vitellogenin; mm, millimeter; g, grams; pg, picograms; mL, milliliter; mg, milligram; ND, not detected; NA, not available]

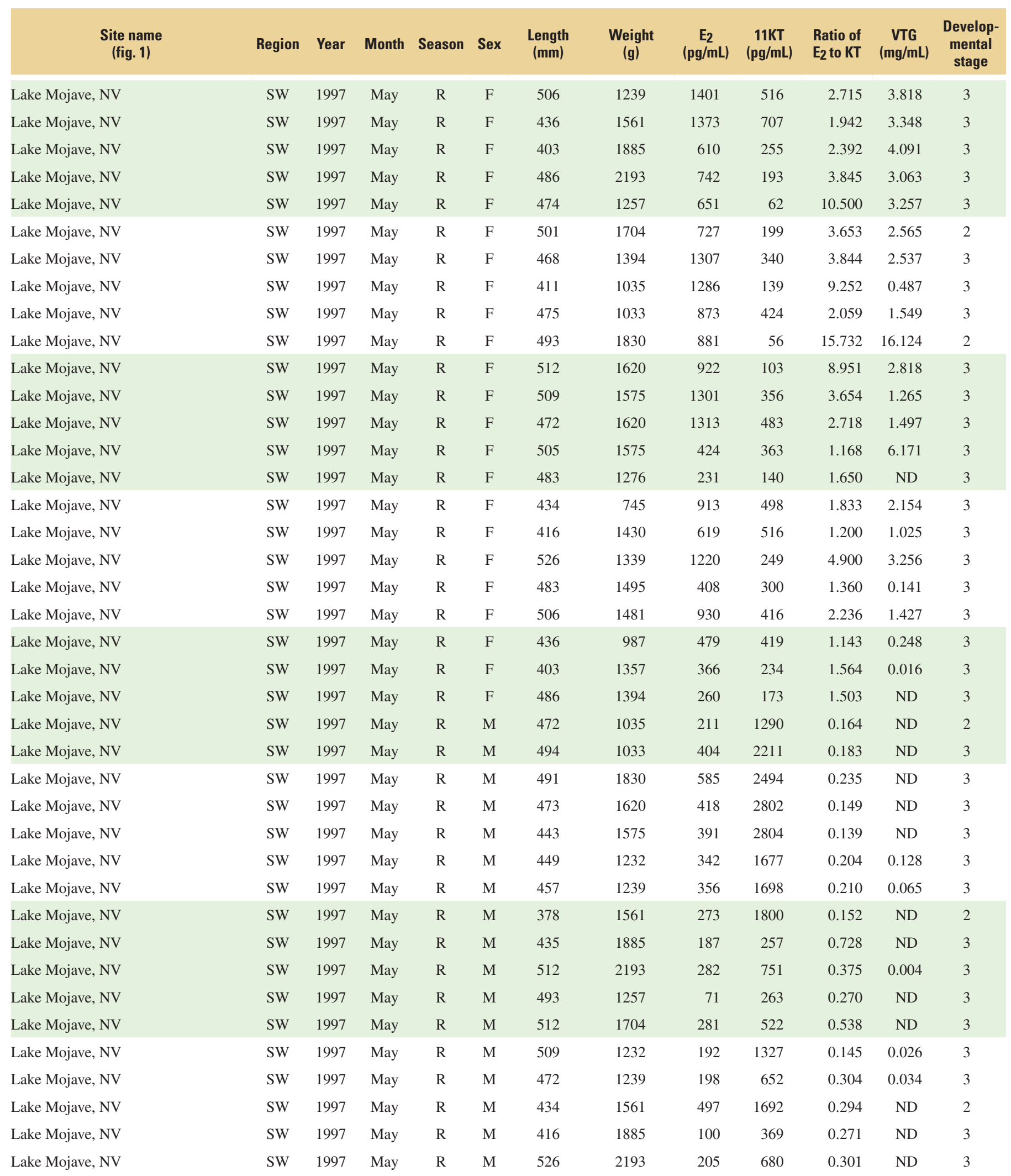


Table 4. (Continued) All individual COMMON CARP values for biomarker and ancillary data from collections made at sites in U.S. waters from August 1994 through October 1997.

[Region: NE, northeast; NW, northwest; SE, southeast; SW, southwest; NMC, northern mid-continent; SMC, southern mid-continent.

Season: NR, non-reproductive. R, reproductive. Sex: F, female, M, male. E2, 17 ß Estradiol; 11KT, 11 ketotestosterone; VTG, vitellogenin;

mm, millimeter; g, grams; pg, picograms; mL, milliliter; mg, milligram; ND, not detected; NA, not available]

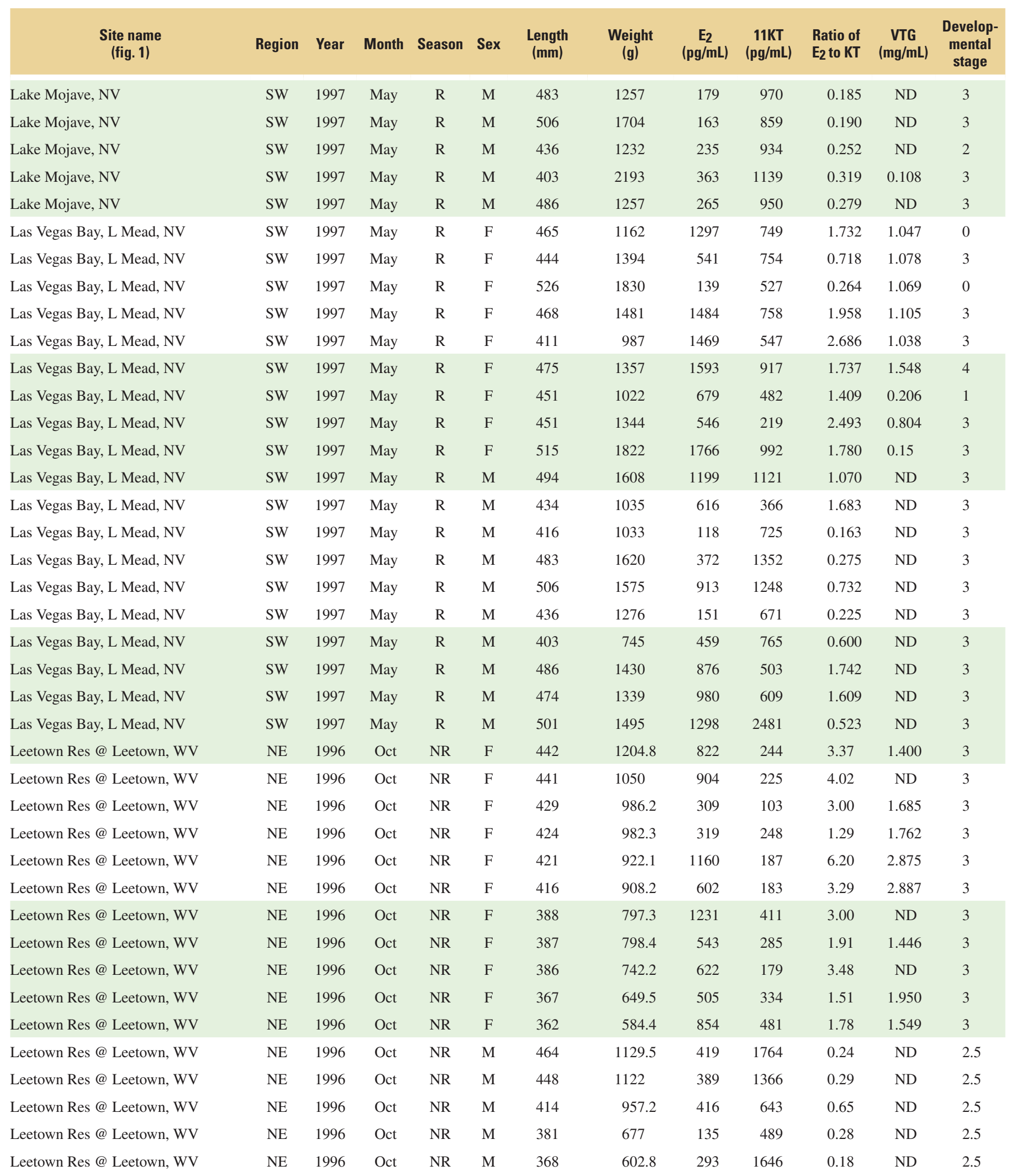


Table 4. (Continued) All individual COMMON CARP values for biomarker and ancillary data from collections made at sites in U.S. waters from August 1994 through October 1997.

[Region: NE, northeast; NW, northwest; SE, southeast; SW, southwest; NMC, northern mid-continent; SMC, southern mid-continent. Season: NR, non-reproductive. R, reproductive. Sex: F, female, M, male. E2, 17 ß Estradiol; 11KT, 11 ketotestosterone; VTG, vitellogenin; mm, millimeter; g, grams; pg, picograms; mL, milliliter; mg, milligram; ND, not detected; NA, not available]

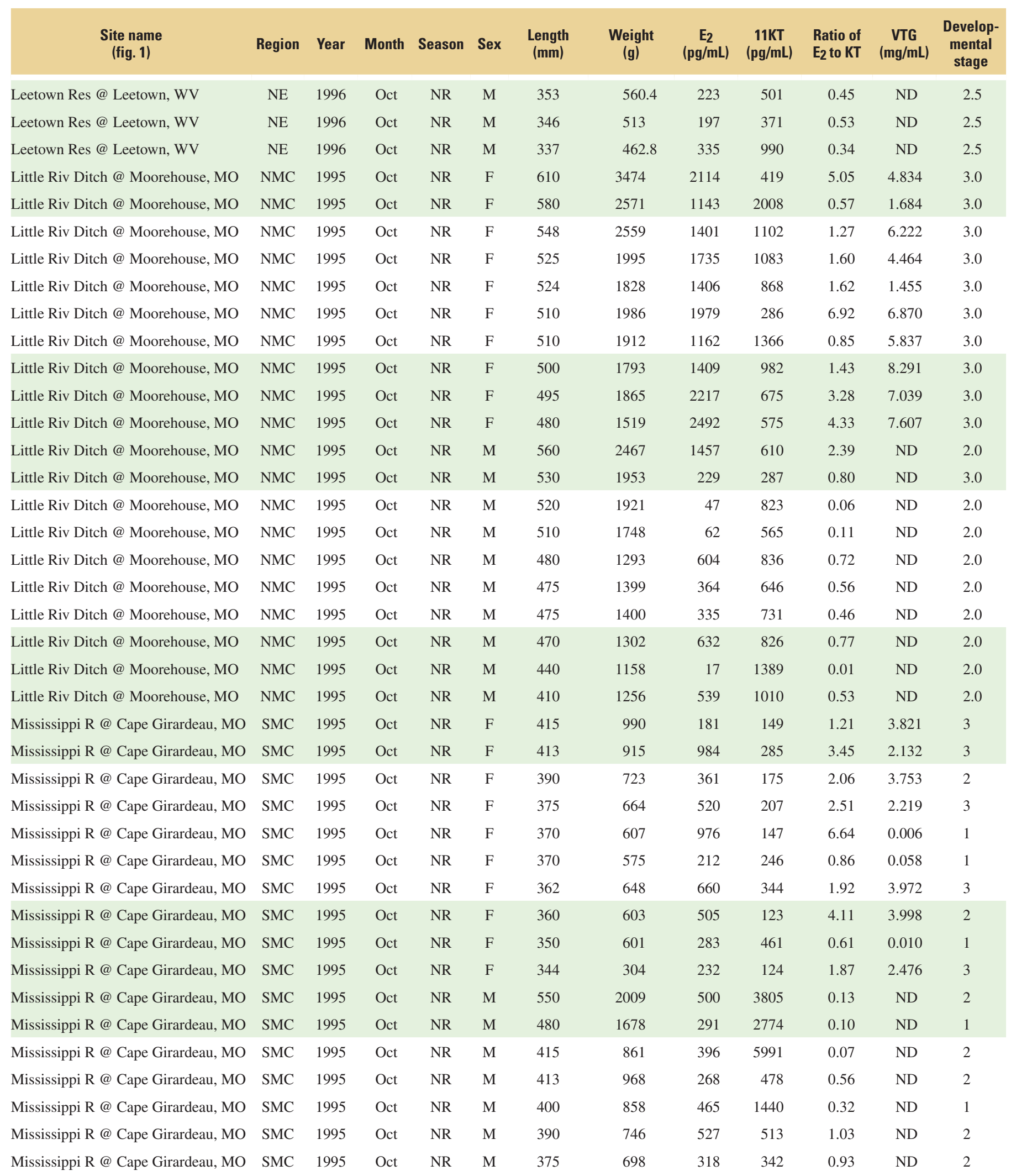


Table 4. (Continued) All individual COMMON CARP values for biomarker and ancillary data from collections made at sites in U.S. waters from August 1994 through October 1997.

[Region: NE, northeast; NW, northwest; SE, southeast; SW, southwest; NMC, northern mid-continent; SMC, southern mid-continent.

Season: NR, non-reproductive. R, reproductive. Sex: F, female, M, male. E2, 17 ß Estradiol; 11KT, 11 ketotestosterone; VTG, vitellogenin;

mm, millimeter; g, grams; pg, picograms; mL, milliliter; mg, milligram; ND, not detected; NA, not available]

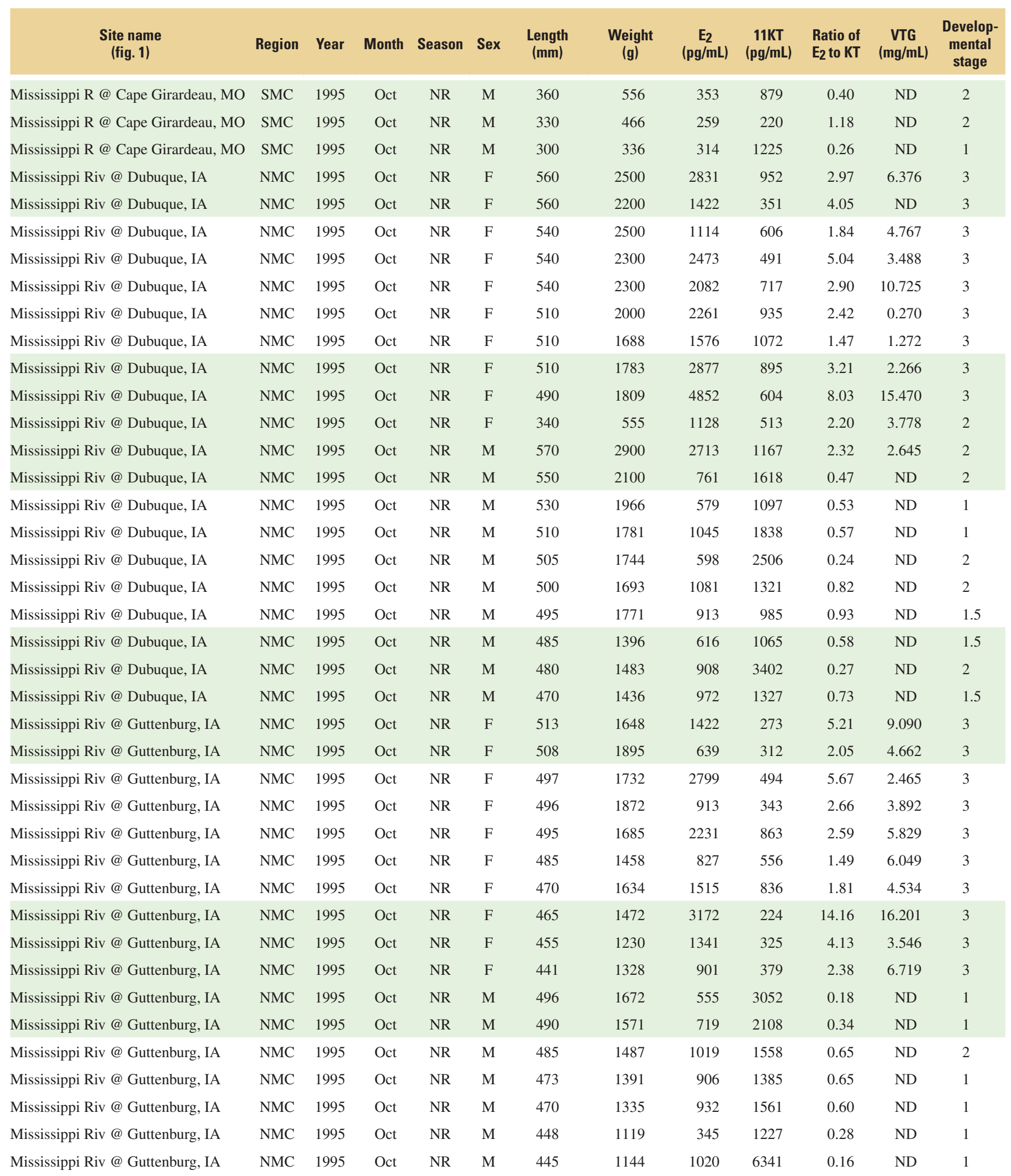


Table 4. (Continued) All individual COMMON CARP values for biomarker and ancillary data from collections made at sites in U.S. waters from August 1994 through October 1997.

[Region: NE, northeast; NW, northwest; SE, southeast; SW, southwest; NMC, northern mid-continent; SMC, southern mid-continent. Season: NR, non-reproductive. R, reproductive. Sex: F, female, M, male. E2, 17 ß Estradiol; 11KT, 11 ketotestosterone; VTG, vitellogenin; mm, millimeter; g, grams; pg, picograms; mL, milliliter; mg, milligram; ND, not detected; NA, not available]

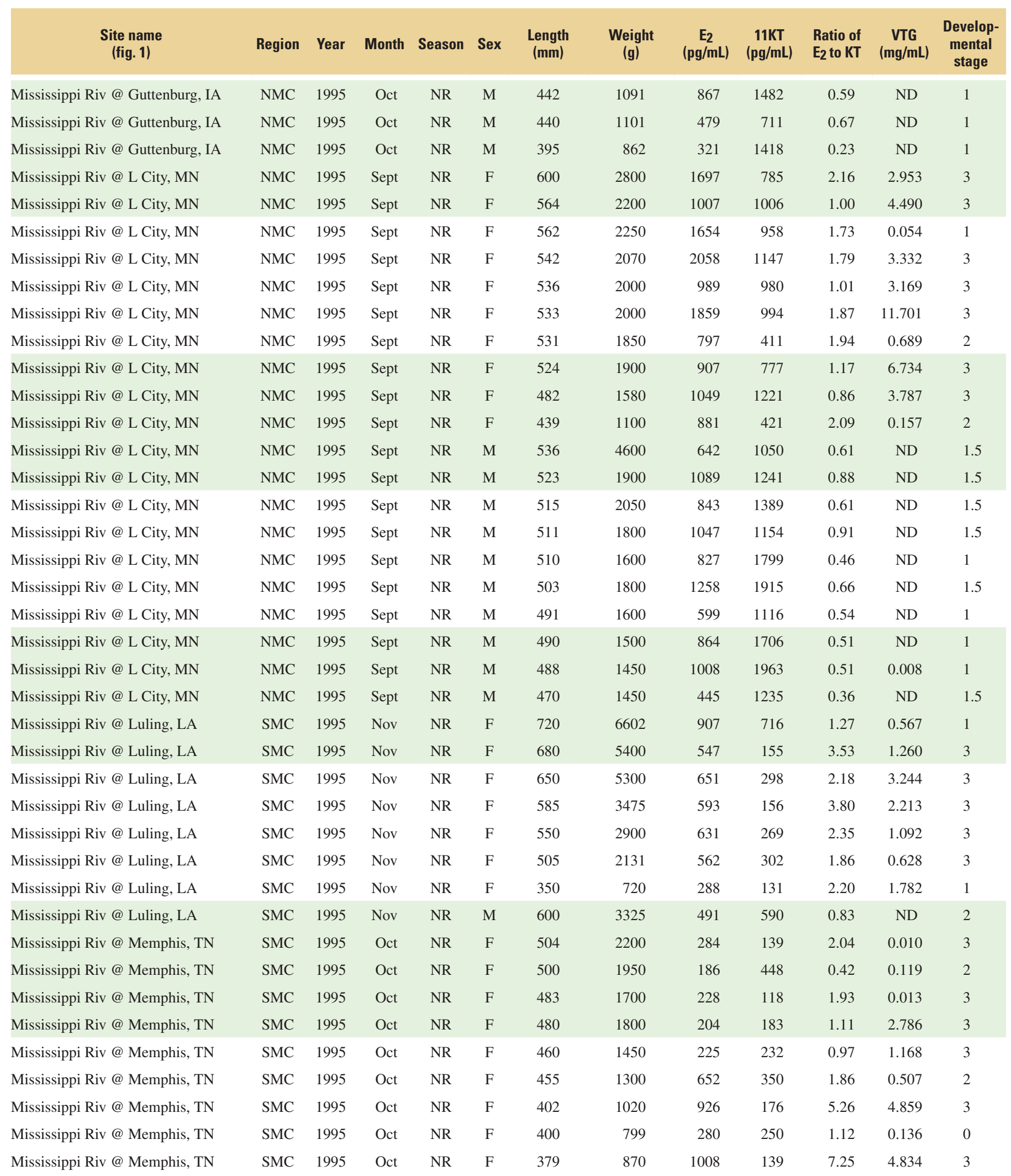


Table 4. (Continued) All individual COMMON CARP values for biomarker and ancillary data from collections made at sites in U.S. waters from August 1994 through October 1997.

[Region: NE, northeast; NW, northwest; SE, southeast; SW, southwest; NMC, northern mid-continent; SMC, southern mid-continent. Season: NR, non-reproductive. R, reproductive. Sex: F, female, M, male. E2, 17 ß Estradiol; 11KT, 11 ketotestosterone; VTG, vitellogenin; mm, millimeter; g, grams; pg, picograms; mL, milliliter; mg, milligram; ND, not detected; NA, not available]

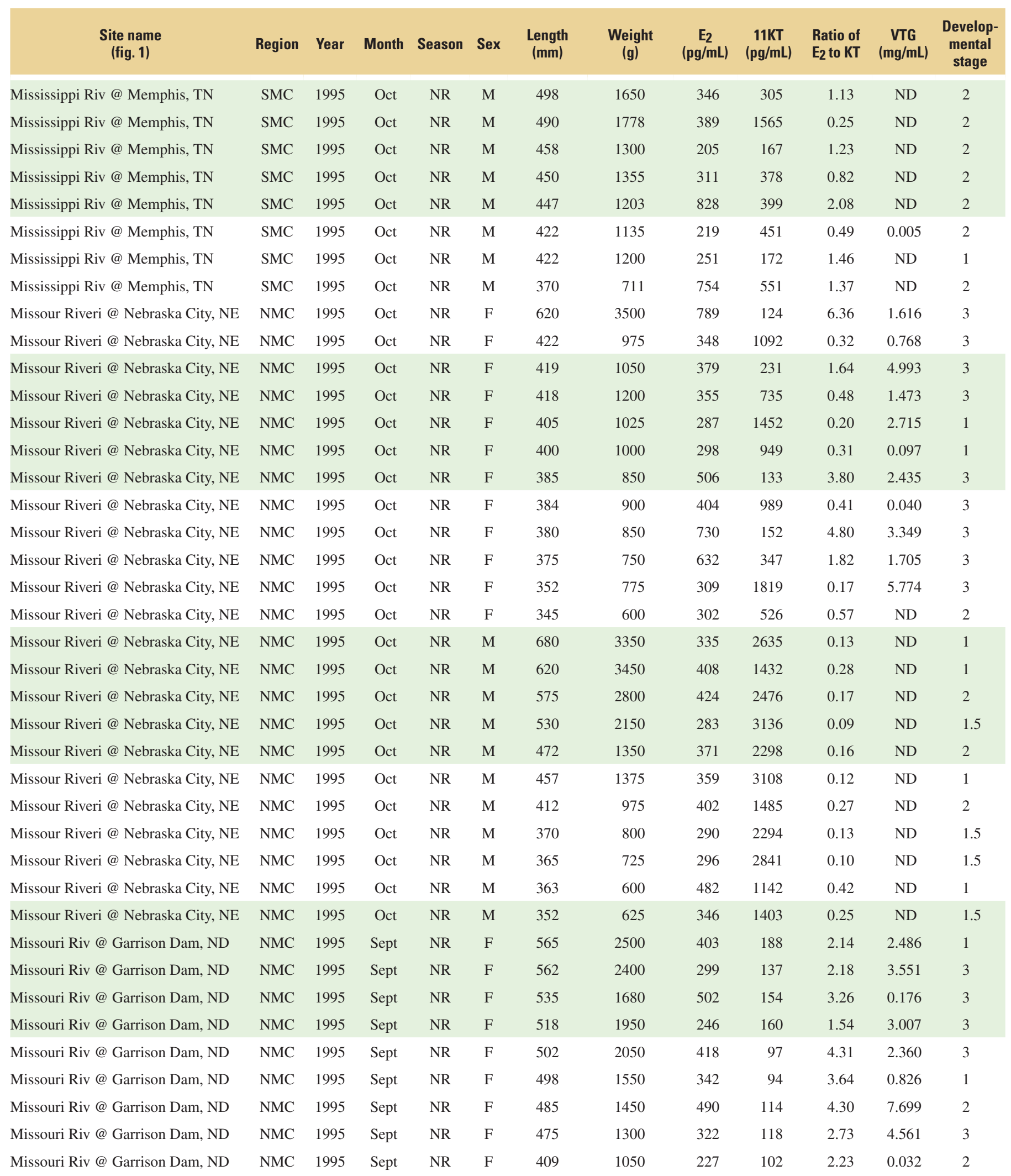


Table 4. (Continued) All individual COMMON CARP values for biomarker and ancillary data from collections made at sites in U.S. waters from August 1994 through October 1997.

[Region: NE, northeast; NW, northwest; SE, southeast; SW, southwest; NMC, northern mid-continent; SMC, southern mid-continent.

Season: NR, non-reproductive. R, reproductive. Sex: F, female, M, male. E2, 17 ß Estradiol; 11KT, 11 ketotestosterone; VTG, vitellogenin;

mm, millimeter; g, grams; pg, picograms; mL, milliliter; mg, milligram; ND, not detected; NA, not available]

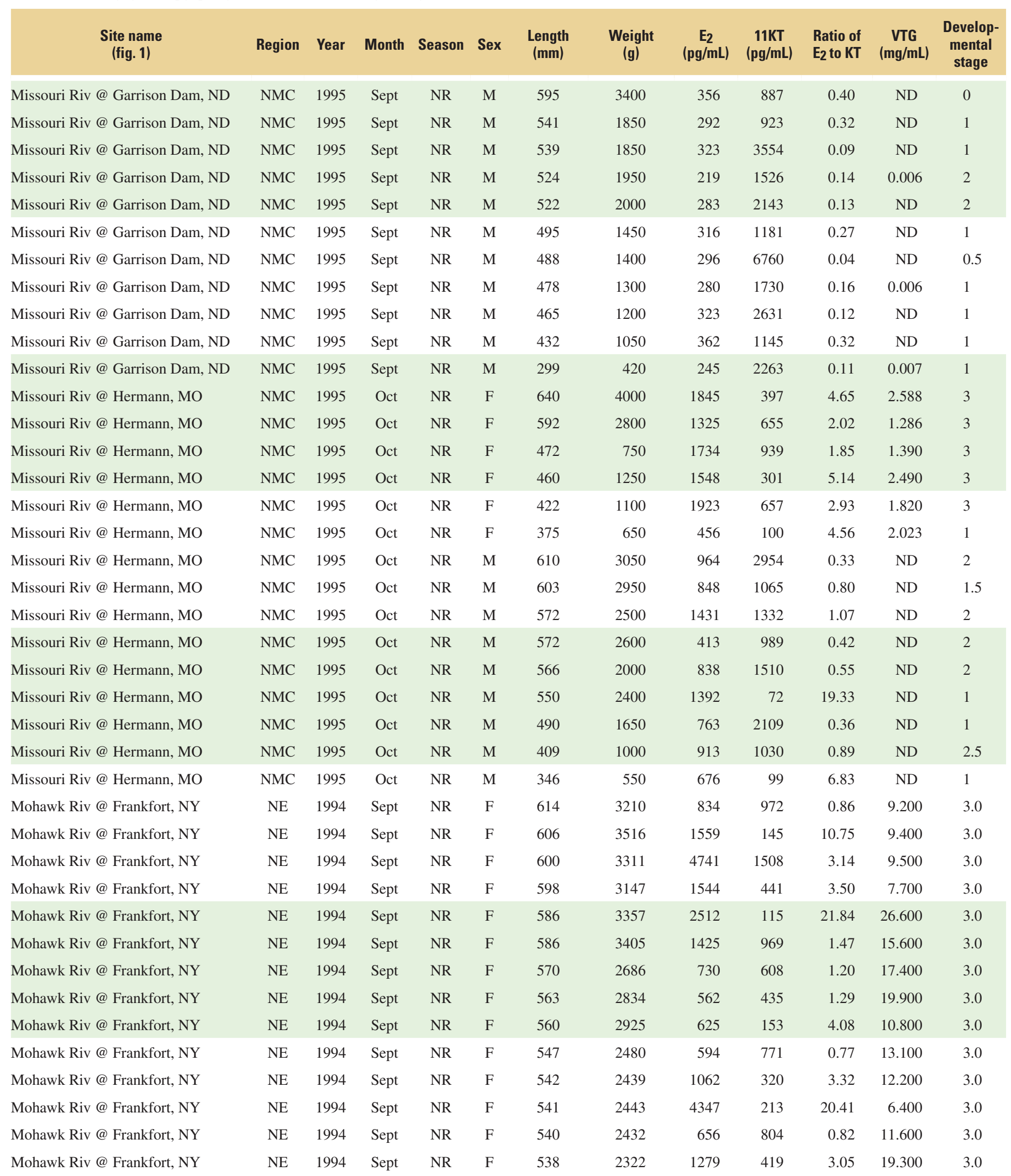


Table 4. (Continued) All individual COMMON CARP values for biomarker and ancillary data from collections made at sites in U.S. waters from August 1994 through October 1997.

[Region: NE, northeast; NW, northwest; SE, southeast; SW, southwest; NMC, northern mid-continent; SMC, southern mid-continent.

Season: NR, non-reproductive. R, reproductive. Sex: F, female, M, male. E2, 17 ß Estradiol; 11KT, 11 ketotestosterone; VTG, vitellogenin;

mm, millimeter; g, grams; pg, picograms; mL, milliliter; mg, milligram; ND, not detected; NA, not available]

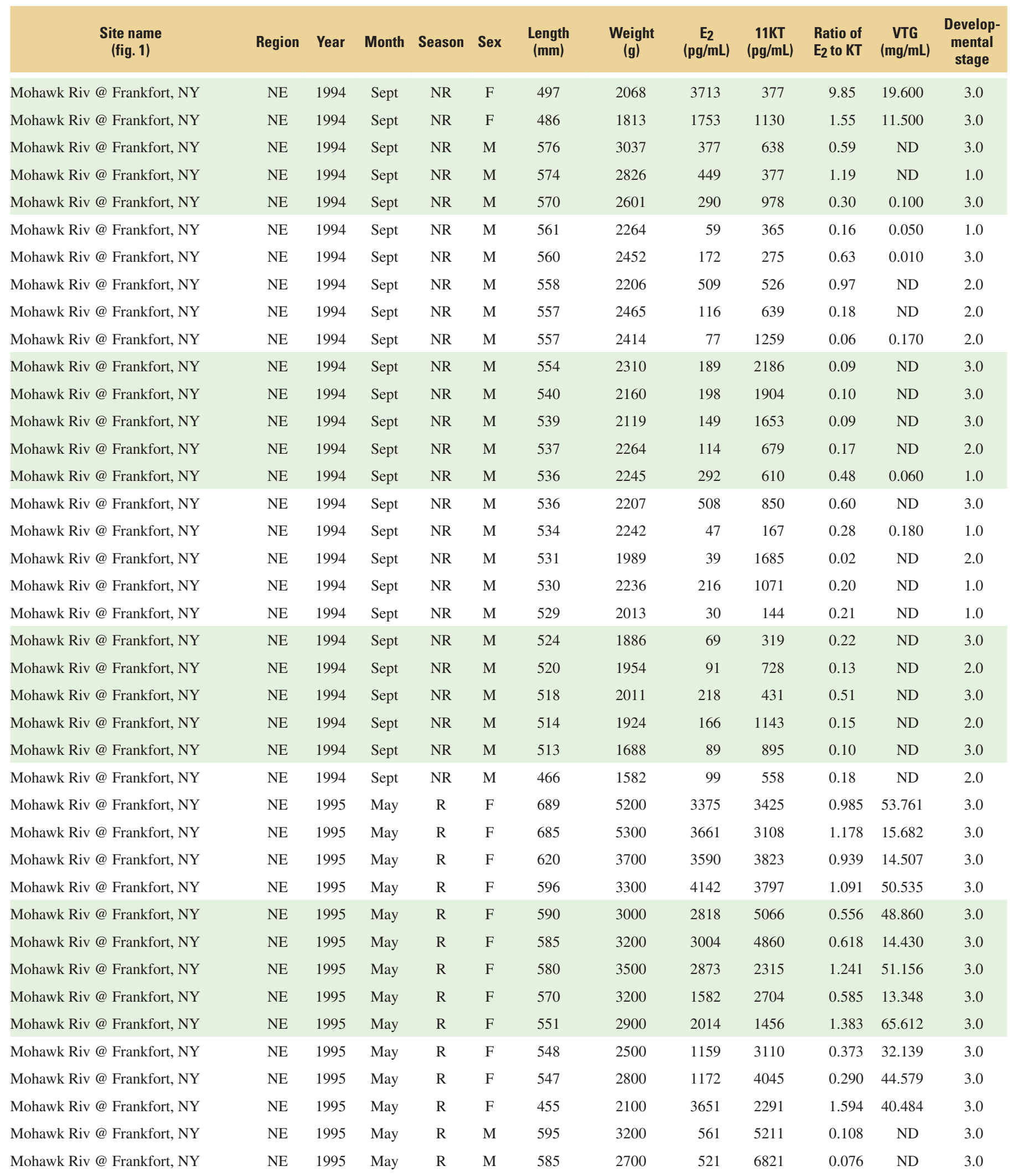


Table 4. (Continued) All individual COMMON CARP values for biomarker and ancillary data from collections made at sites in U.S. waters from August 1994 through October 1997.

[Region: NE, northeast; NW, northwest; SE, southeast; SW, southwest; NMC, northern mid-continent; SMC, southern mid-continent.

Season: NR, non-reproductive. R, reproductive. Sex: F, female, M, male. E2, 17 ß Estradiol; 11KT, 11 ketotestosterone; VTG, vitellogenin;

mm, millimeter; g, grams; pg, picograms; mL, milliliter; mg, milligram; ND, not detected; NA, not available]

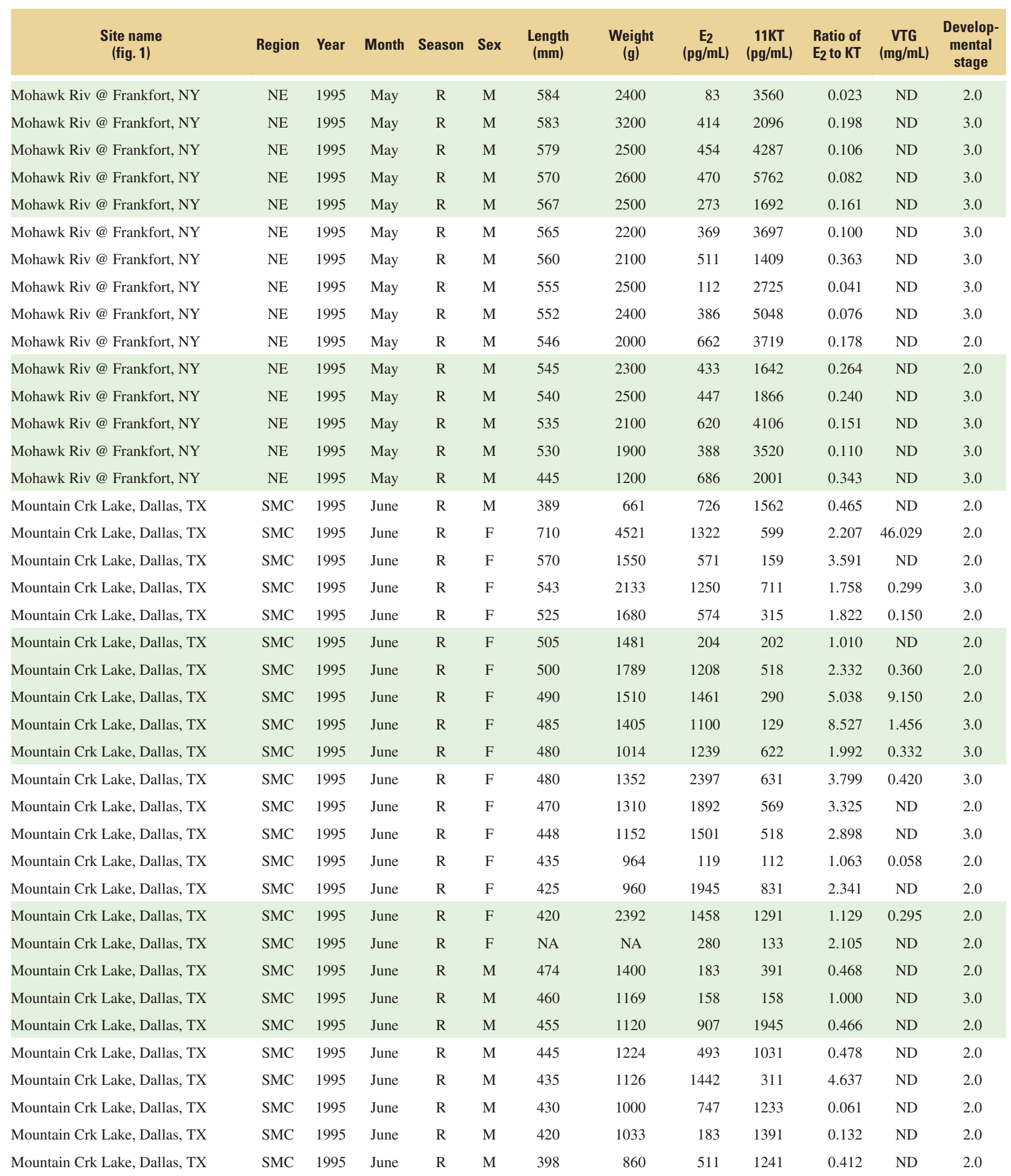


Table 4. (Continued) All individual COMMON CARP values for biomarker and ancillary data from collections made at sites in U.S. waters from August 1994 through October 1997.

[Region: NE, northeast; NW, northwest; SE, southeast; SW, southwest; NMC, northern mid-continent; SMC, southern mid-continent.

Season: NR, non-reproductive. R, reproductive. Sex: F, female, M, male. E2, 17 ß Estradiol; 11KT, 11 ketotestosterone; VTG, vitellogenin; mm, millimeter; g, grams; pg, picograms; mL, milliliter; mg, milligram; ND, not detected; NA, not available]

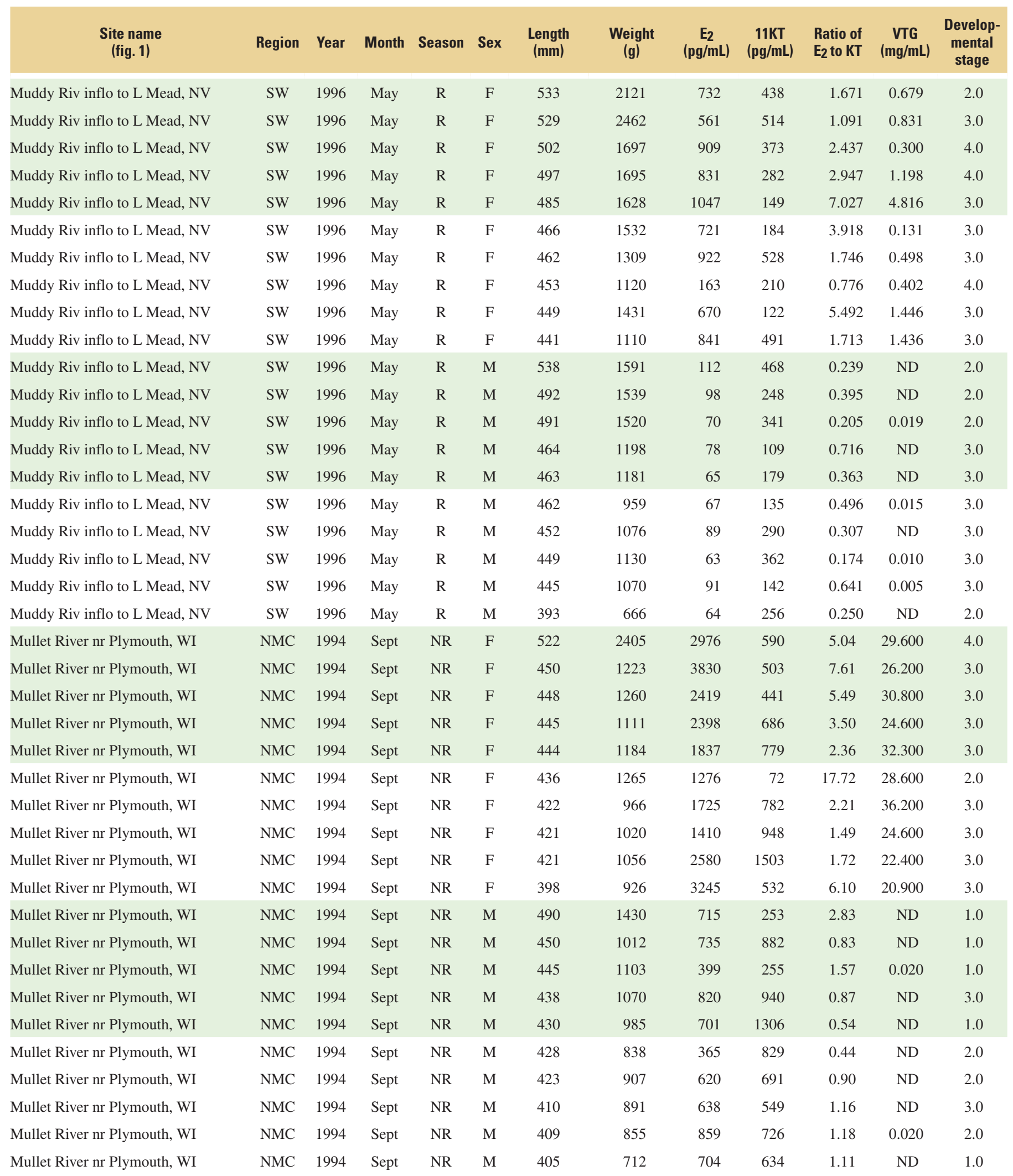


Table 4. (Continued) All individual COMMON CARP values for biomarker and ancillary data from collections made at sites in U.S. waters from August 1994 through October 1997.

[Region: NE, northeast; NW, northwest; SE, southeast; SW, southwest; NMC, northern mid-continent; SMC, southern mid-continent.

Season: NR, non-reproductive. R, reproductive. Sex: F, female, M, male. E2, 17 ß Estradiol; 11KT, 11 ketotestosterone; VTG, vitellogenin; mm, millimeter; g, grams; pg, picograms; mL, milliliter; mg, milligram; ND, not detected; NA, not available]

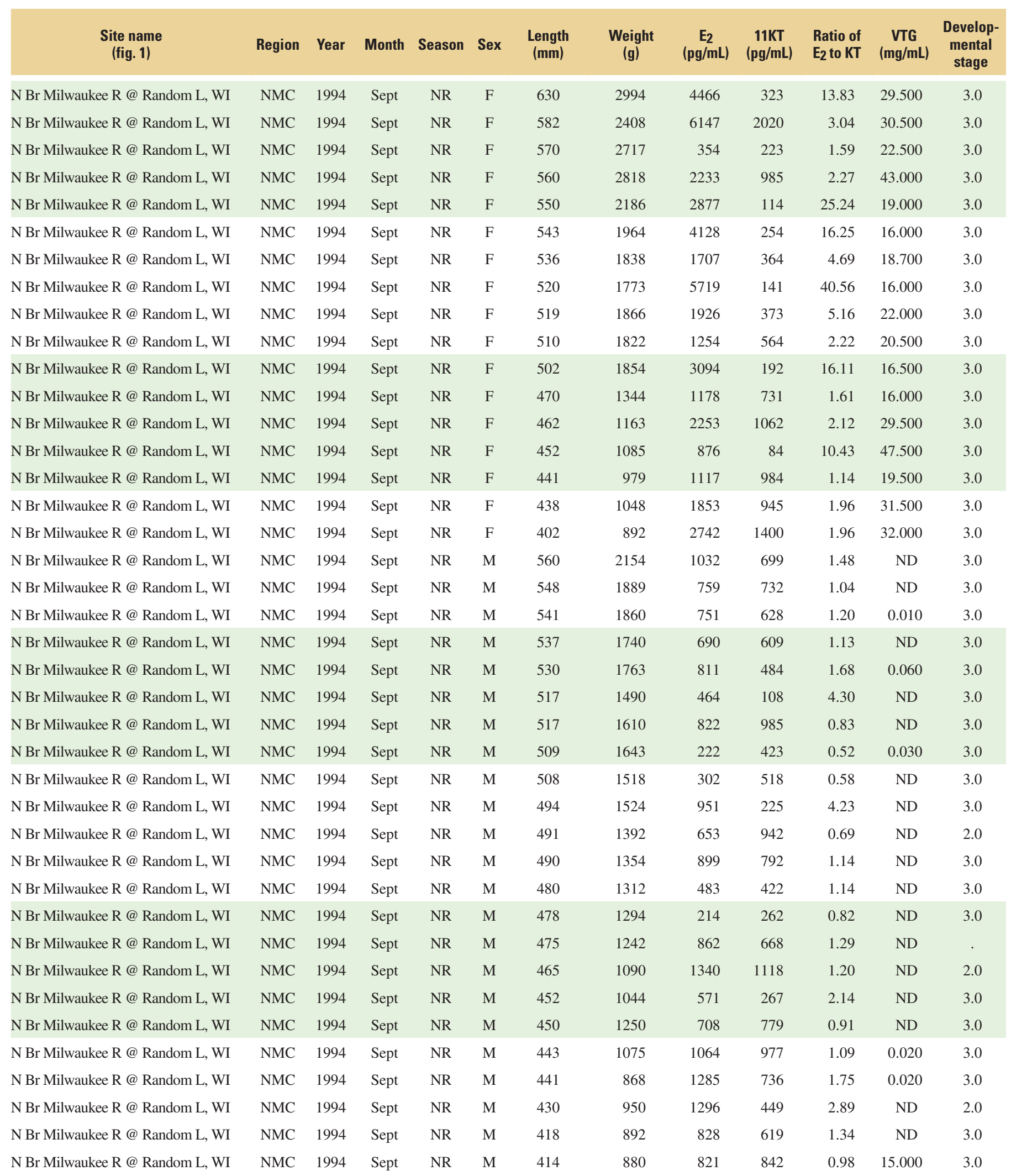


Table 4. (Continued) All individual COMMON CARP values for biomarker and ancillary data from collections made at sites in U.S. waters from August 1994 through October 1997.

[Region: NE, northeast; NW, northwest; SE, southeast; SW, southwest; NMC, northern mid-continent; SMC, southern mid-continent. Season: NR, non-reproductive. R, reproductive. Sex: F, female, M, male. E2, 17 ß Estradiol; 11KT, 11 ketotestosterone; VTG, vitellogenin; mm, millimeter; g, grams; pg, picograms; mL, milliliter; mg, milligram; ND, not detected; NA, not available]

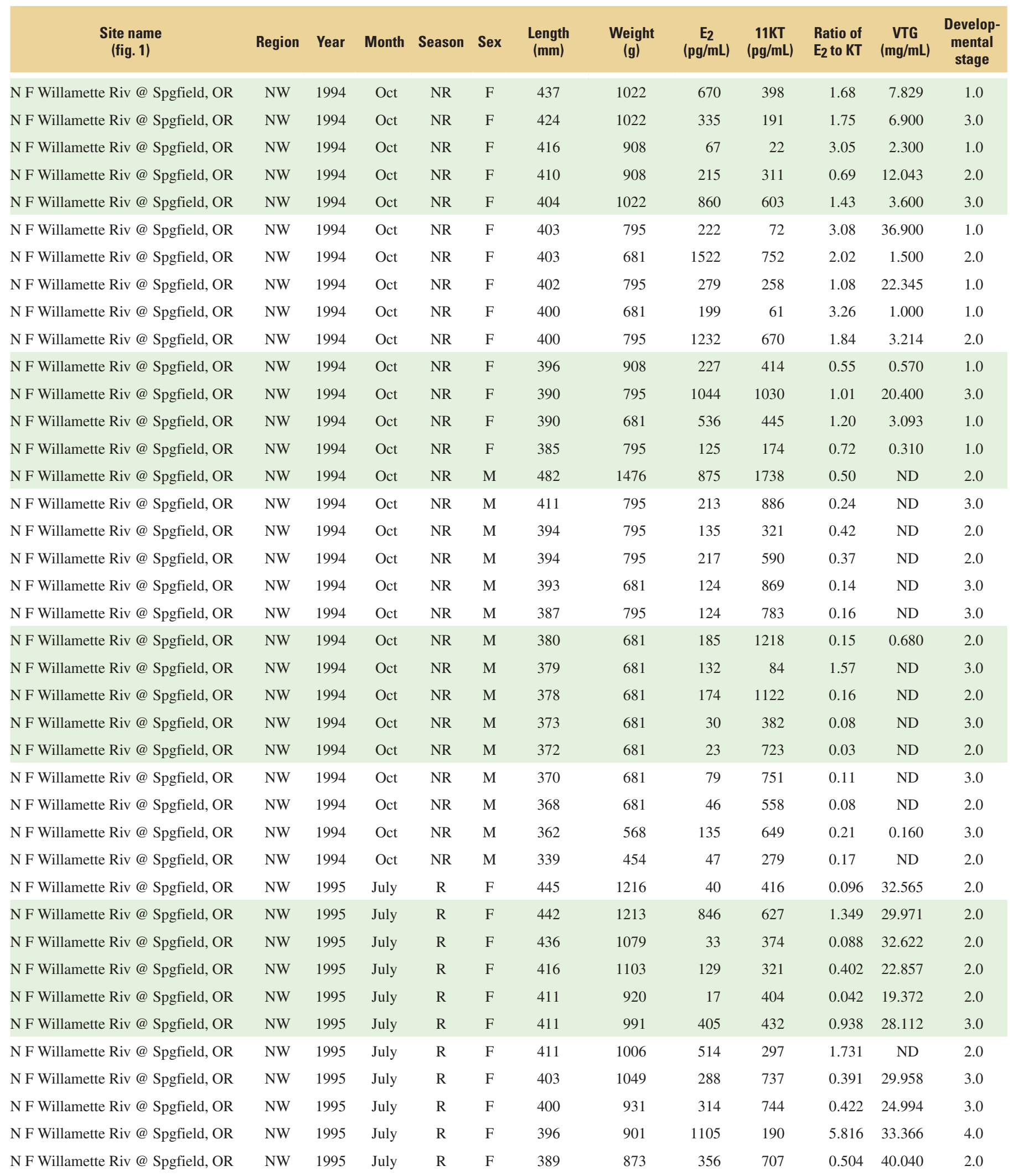


Table 4. (Continued) All individual COMMON CARP values for biomarker and ancillary data from collections made at sites in U.S. waters from August 1994 through October 1997.

[Region: NE, northeast; NW, northwest; SE, southeast; SW, southwest; NMC, northern mid-continent; SMC, southern mid-continent. Season: NR, non-reproductive. R, reproductive. Sex: F, female, M, male. E2, 17 ß Estradiol; 11KT, 11 ketotestosterone; VTG, vitellogenin; mm, millimeter; g, grams; pg, picograms; mL, milliliter; mg, milligram; ND, not detected; NA, not available]

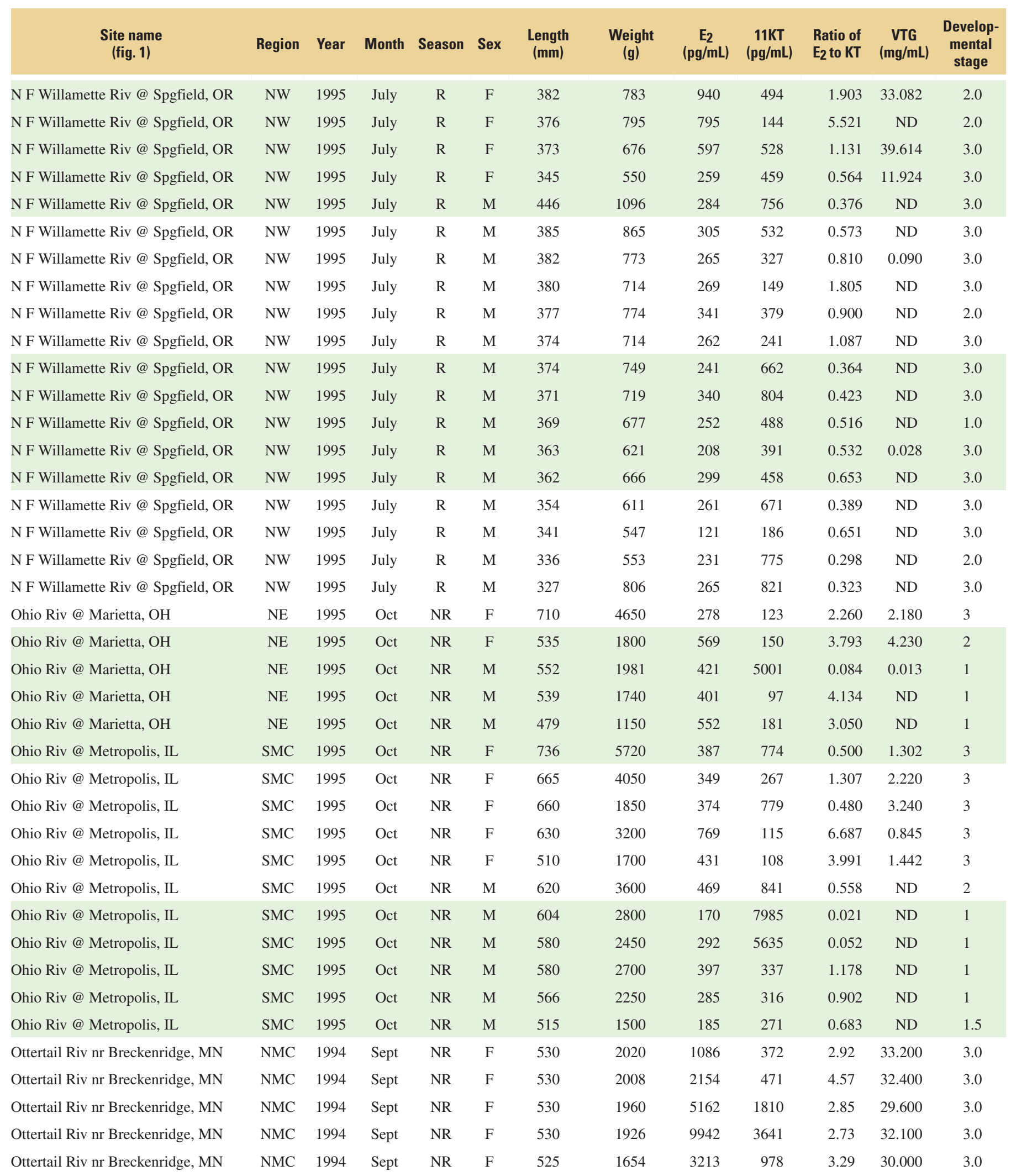


Table 4. (Continued) All individual COMMON CARP values for biomarker and ancillary data from collections made at sites in U.S. waters from August 1994 through October 1997.

[Region: NE, northeast; NW, northwest; SE, southeast; SW, southwest; NMC, northern mid-continent; SMC, southern mid-continent.

Season: NR, non-reproductive. R, reproductive. Sex: F, female, M, male. E2, 17 ß Estradiol; 11KT, 11 ketotestosterone; VTG, vitellogenin; mm, millimeter; g, grams; pg, picograms; mL, milliliter; mg, milligram; ND, not detected; NA, not available]

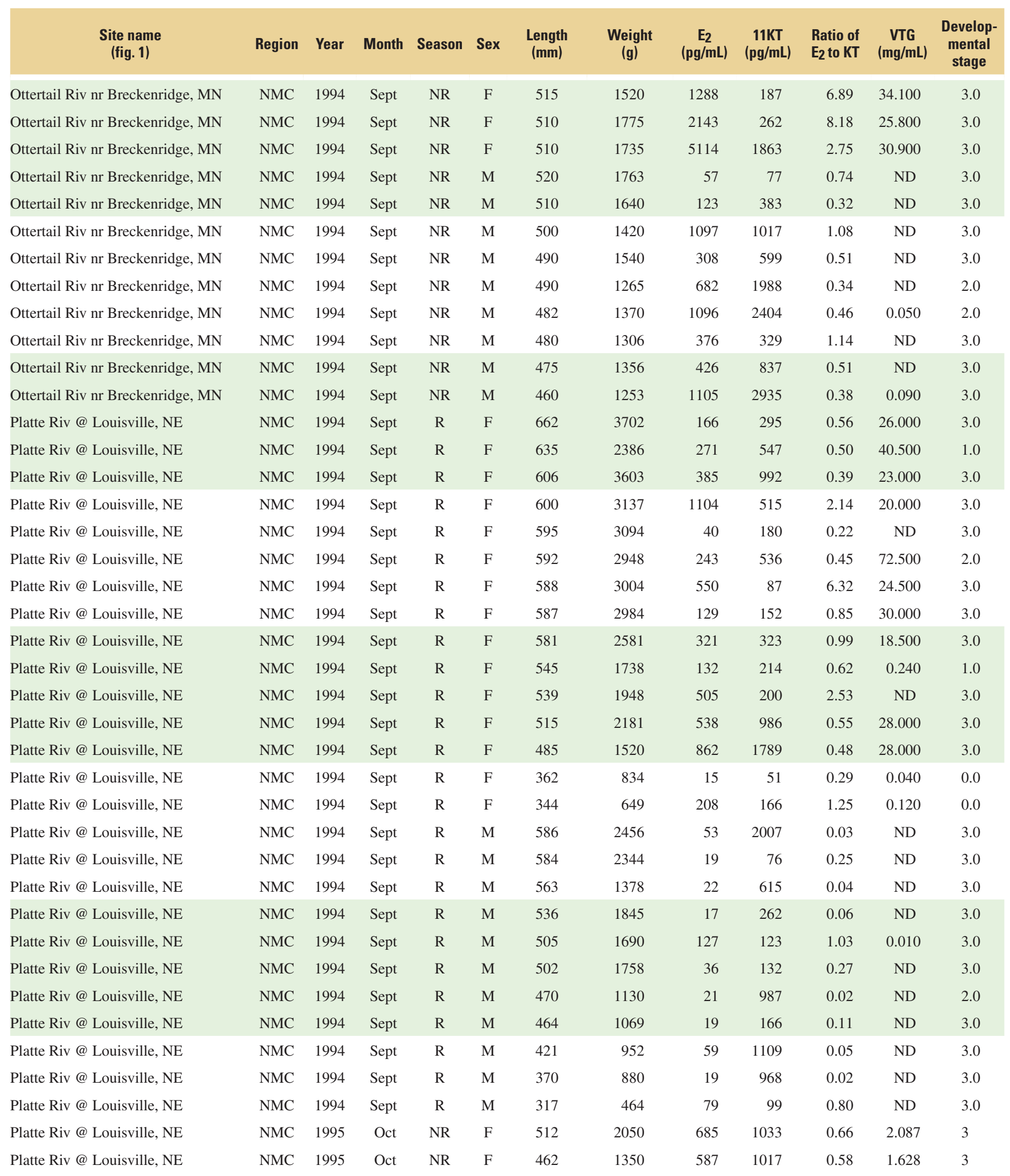


Table 4. (Continued) All individual COMMON CARP values for biomarker and ancillary data from collections made at sites in U.S. waters from August 1994 through October 1997.

[Region: NE, northeast; NW, northwest; SE, southeast; SW, southwest; NMC, northern mid-continent; SMC, southern mid-continent. Season: NR, non-reproductive. R, reproductive. Sex: F, female, M, male. E2, 17 ß Estradiol; 11KT, 11 ketotestosterone; VTG, vitellogenin; mm, millimeter; g, grams; pg, picograms; mL, milliliter; mg, milligram; ND, not detected; NA, not available]

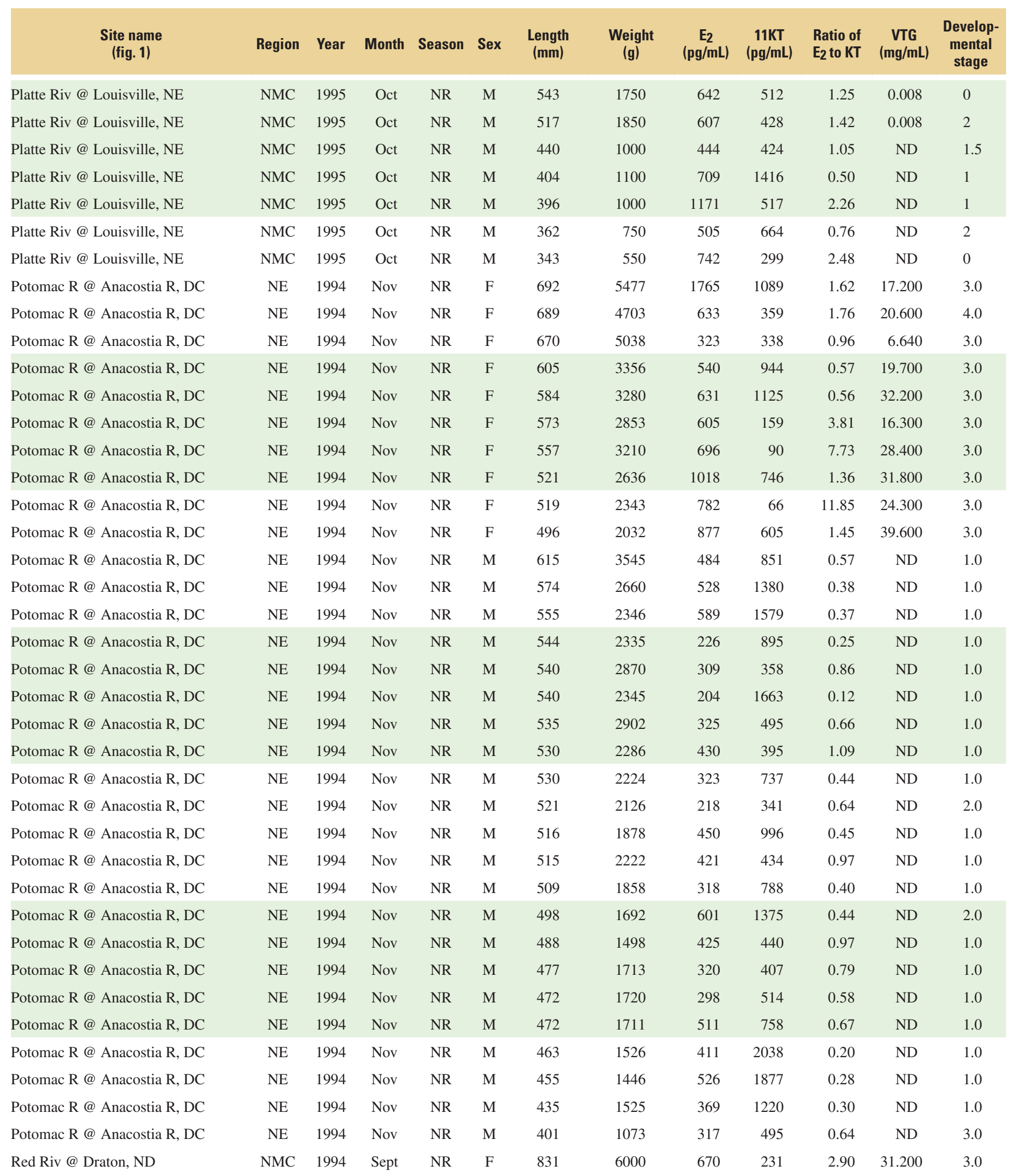


Table 4. (Continued) All individual COMMON CARP values for biomarker and ancillary data from collections made at sites in U.S. waters from August 1994 through October 1997.

[Region: NE, northeast; NW, northwest; SE, southeast; SW, southwest; NMC, northern mid-continent; SMC, southern mid-continent.

Season: NR, non-reproductive. R, reproductive. Sex: F, female, M, male. E2, 17 ß Estradiol; 11KT, 11 ketotestosterone; VTG, vitellogenin; mm, millimeter; g, grams; pg, picograms; mL, milliliter; mg, milligram; ND, not detected; NA, not available]

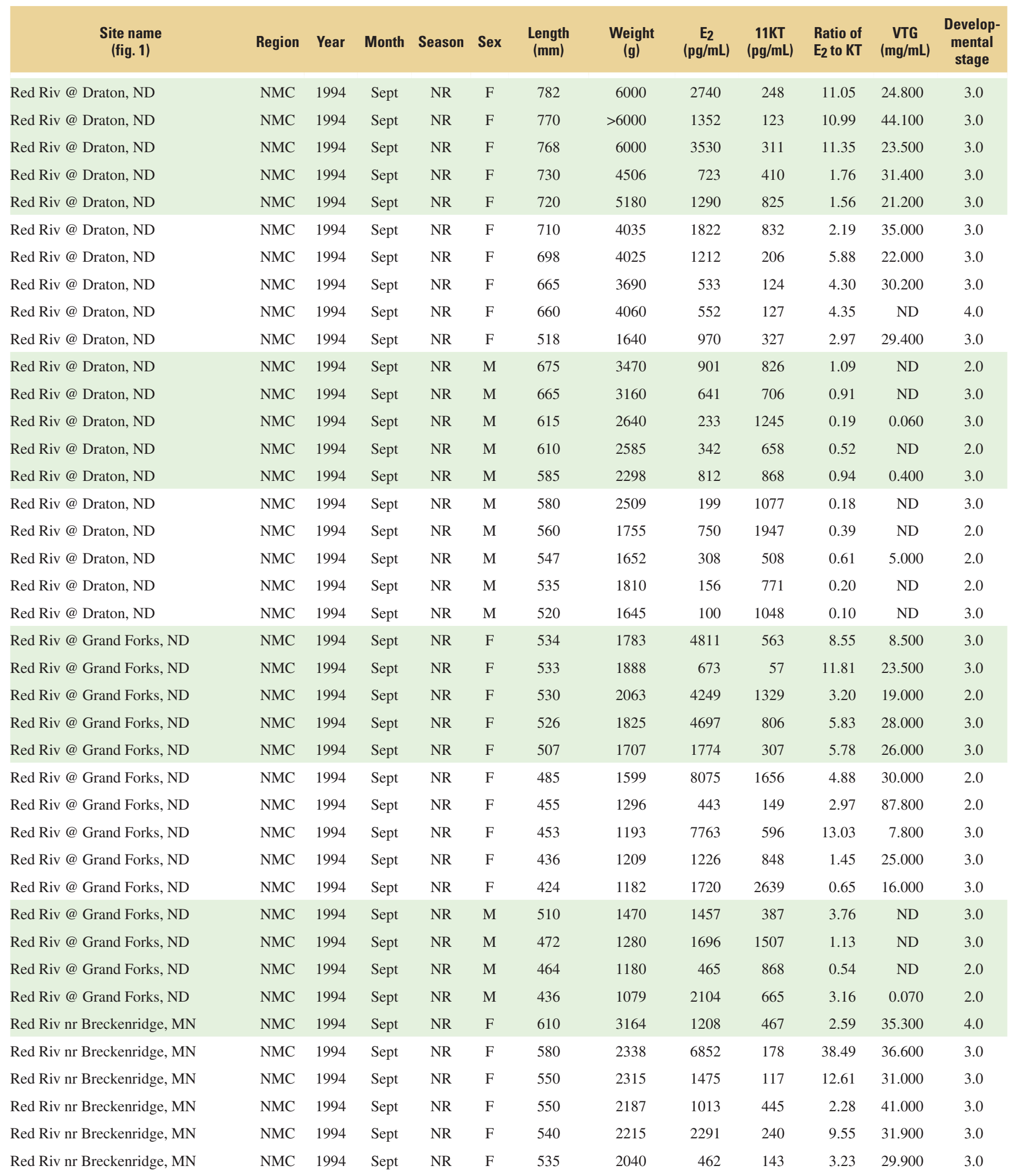


Table 4. (Continued) All individual COMMON CARP values for biomarker and ancillary data from collections made at sites in U.S. waters from August 1994 through October 1997.

[Region: NE, northeast; NW, northwest; SE, southeast; SW, southwest; NMC, northern mid-continent; SMC, southern mid-continent. Season: NR, non-reproductive. R, reproductive. Sex: F, female, M, male. E2, 17 ß Estradiol; 11KT, 11 ketotestosterone; VTG, vitellogenin; mm, millimeter; g, grams; pg, picograms; mL, milliliter; mg, milligram; ND, not detected; NA, not available]

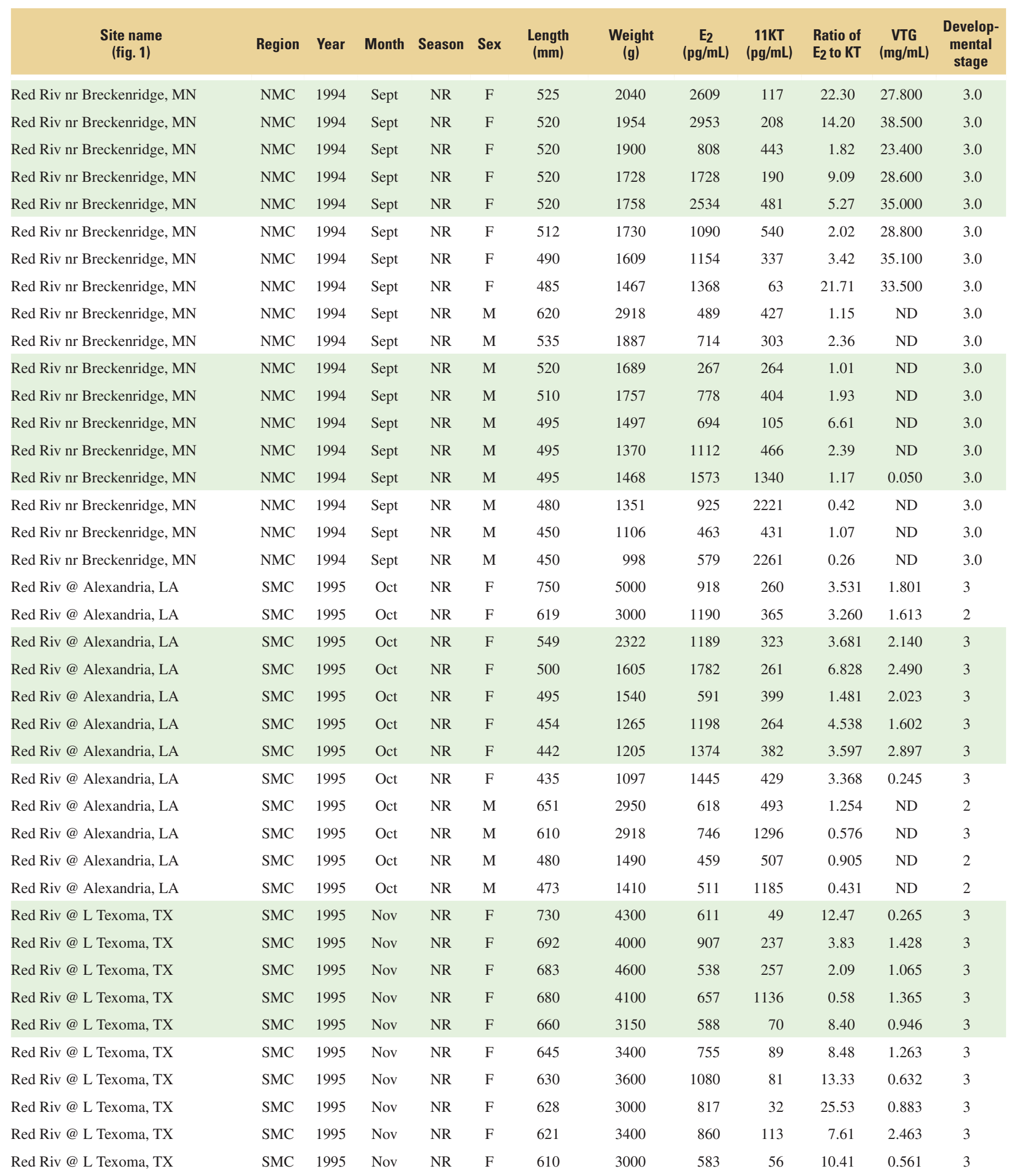


Table 4. (Continued) All individual COMMON CARP values for biomarker and ancillary data from collections made at sites in U.S. waters from August 1994 through October 1997.

[Region: NE, northeast; NW, northwest; SE, southeast; SW, southwest; NMC, northern mid-continent; SMC, southern mid-continent.

Season: NR, non-reproductive. R, reproductive. Sex: F, female, M, male. E2, 17 ß Estradiol; 11KT, 11 ketotestosterone; VTG, vitellogenin; mm, millimeter; g, grams; pg, picograms; mL, milliliter; mg, milligram; ND, not detected; NA, not available]

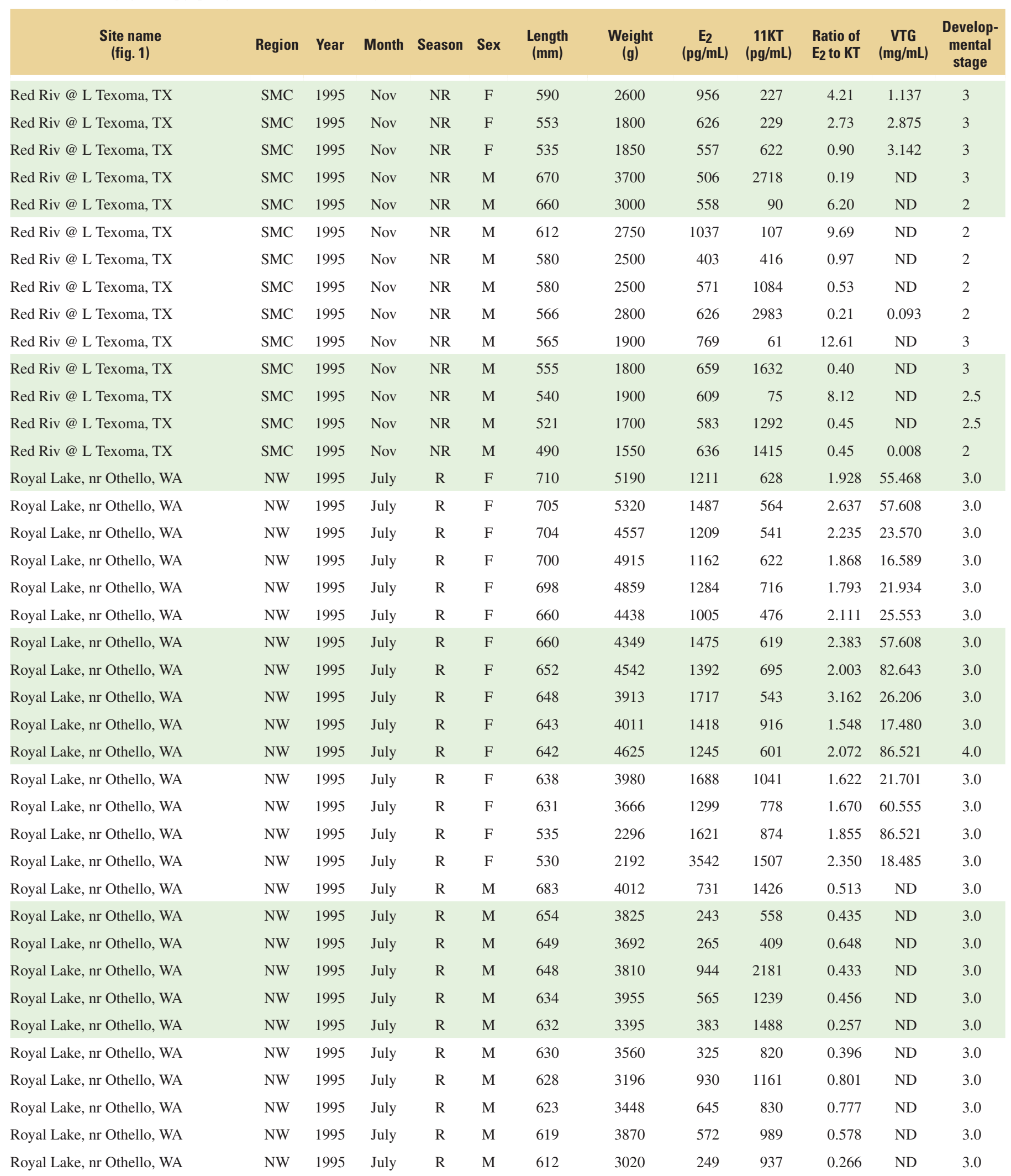


Table 4. (Continued) All individual COMMON CARP values for biomarker and ancillary data from collections made at sites in U.S. waters from August 1994 through October 1997.

[Region: NE, northeast; NW, northwest; SE, southeast; SW, southwest; NMC, northern mid-continent; SMC, southern mid-continent.

Season: NR, non-reproductive. R, reproductive. Sex: F, female, M, male. E2, 17 ß Estradiol; 11KT, 11 ketotestosterone; VTG, vitellogenin;

mm, millimeter; g, grams; pg, picograms; mL, milliliter; mg, milligram; ND, not detected; NA, not available]

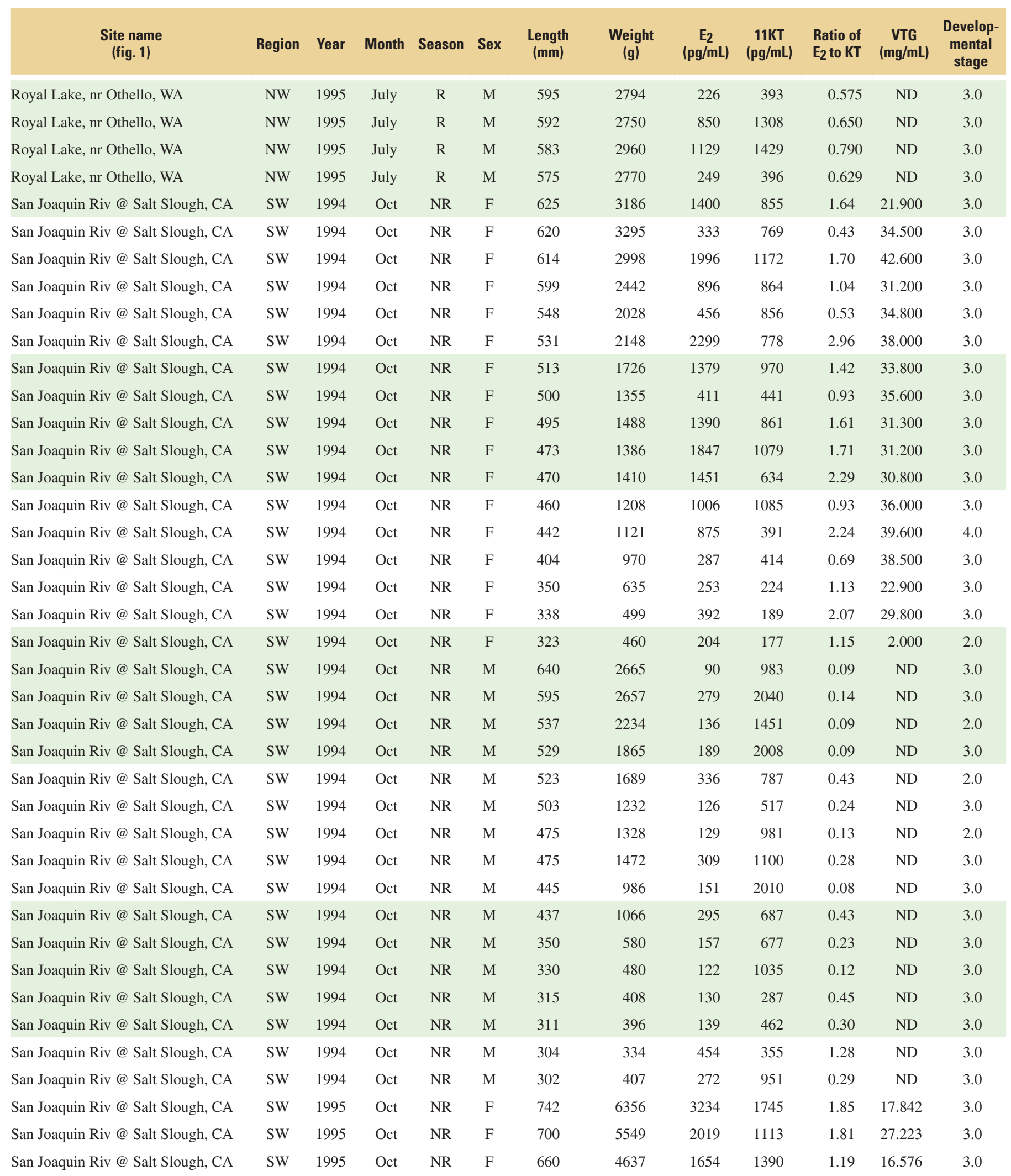


Table 4. (Continued) All individual COMMON CARP values for biomarker and ancillary data from collections made at sites in U.S. waters from August 1994 through October 1997.

[Region: NE, northeast; NW, northwest; SE, southeast; SW, southwest; NMC, northern mid-continent; SMC, southern mid-continent.

Season: NR, non-reproductive. R, reproductive. Sex: F, female, M, male. E2, 17 ß Estradiol; 11KT, 11 ketotestosterone; VTG, vitellogenin; mm, millimeter; g, grams; pg, picograms; mL, milliliter; mg, milligram; ND, not detected; NA, not available]

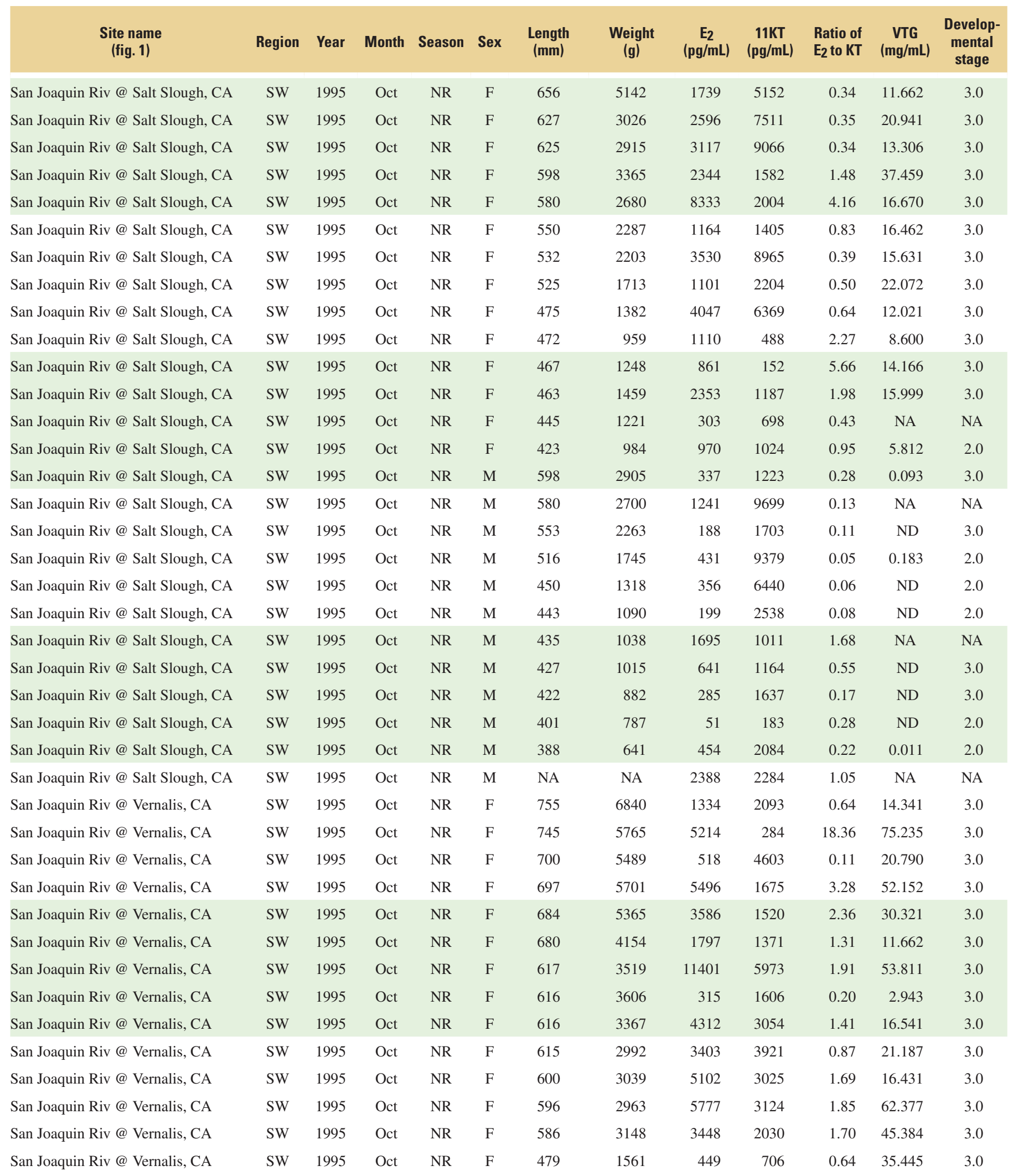


Table 4. (Continued) All individual COMMON CARP values for biomarker and ancillary data from collections made at sites in U.S. waters from August 1994 through October 1997.

[Region: NE, northeast; NW, northwest; SE, southeast; SW, southwest; NMC, northern mid-continent; SMC, southern mid-continent.

Season: NR, non-reproductive. R, reproductive. Sex: F, female, M, male. E2, 17 ß Estradiol; 11KT, 11 ketotestosterone; VTG, vitellogenin;

mm, millimeter; g, grams; pg, picograms; mL, milliliter; mg, milligram; ND, not detected; NA, not available]

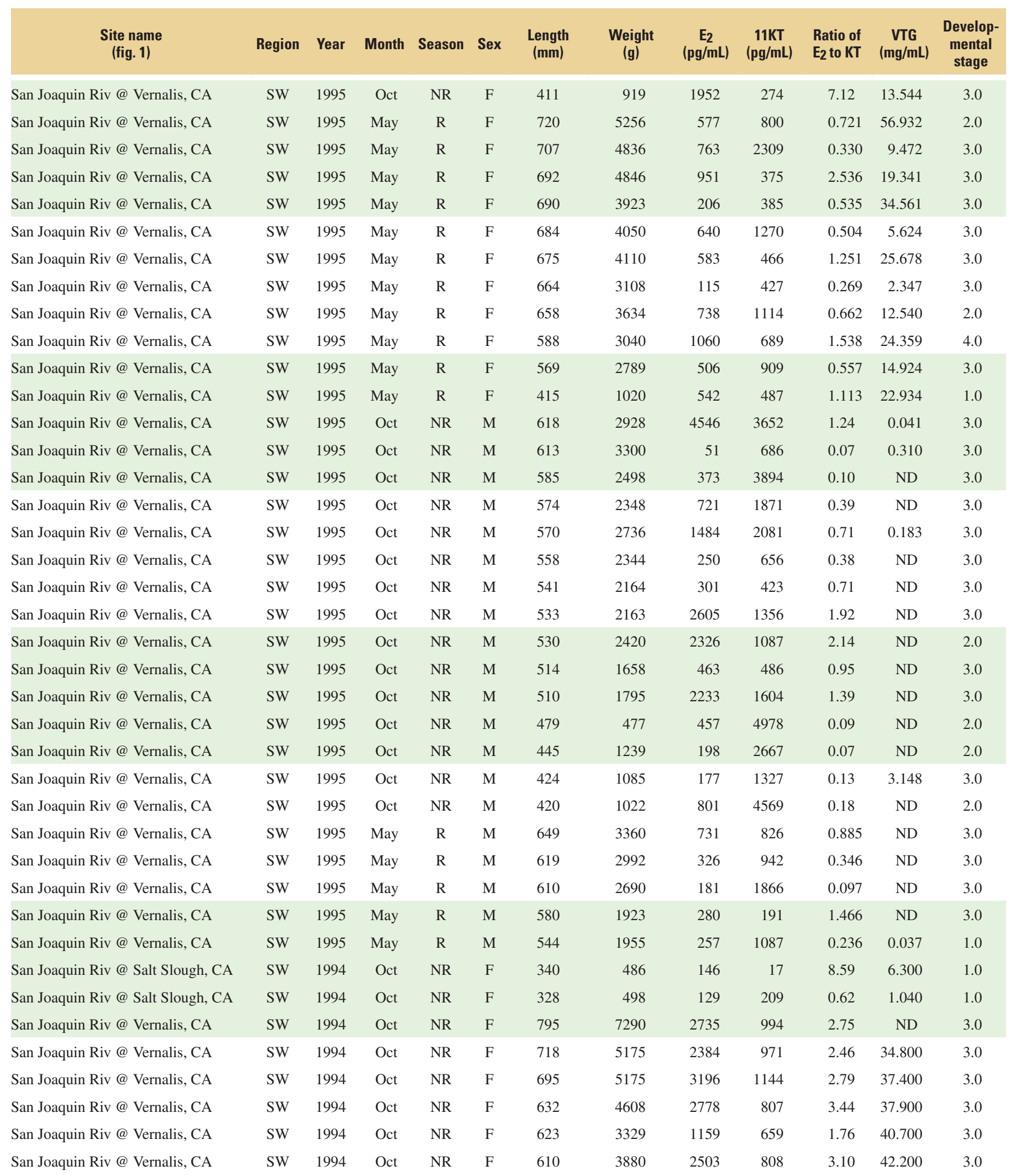


Table 4. (Continued) All individual COMMON CARP values for biomarker and ancillary data from collections made at sites in U.S. waters from August 1994 through October 1997.

[Region: NE, northeast; NW, northwest; SE, southeast; SW, southwest; NMC, northern mid-continent; SMC, southern mid-continent.

Season: NR, non-reproductive. R, reproductive. Sex: F, female, M, male. E2, 17 ß Estradiol; 11KT, 11 ketotestosterone; VTG, vitellogenin; mm, millimeter; g, grams; pg, picograms; mL, milliliter; mg, milligram; ND, not detected; NA, not available]

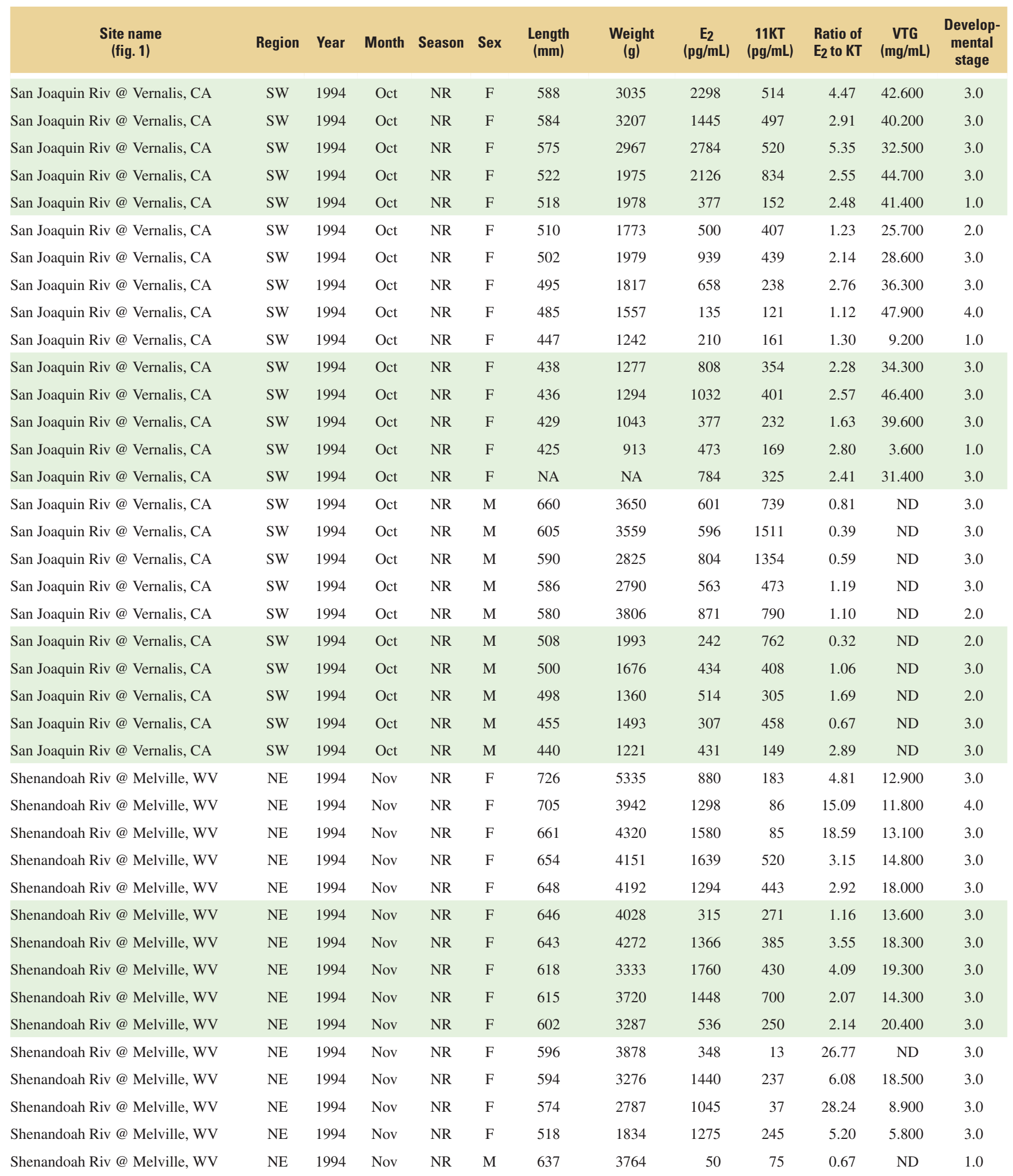


Table 4. (Continued) All individual COMMON CARP values for biomarker and ancillary data from collections made at sites in U.S. waters from August 1994 through October 1997.

[Region: NE, northeast; NW, northwest; SE, southeast; SW, southwest; NMC, northern mid-continent; SMC, southern mid-continent. Season: NR, non-reproductive. R, reproductive. Sex: F, female, M, male. E2, 17 ß Estradiol; 11KT, 11 ketotestosterone; VTG, vitellogenin; mm, millimeter; g, grams; pg, picograms; mL, milliliter; mg, milligram; ND, not detected; NA, not available]

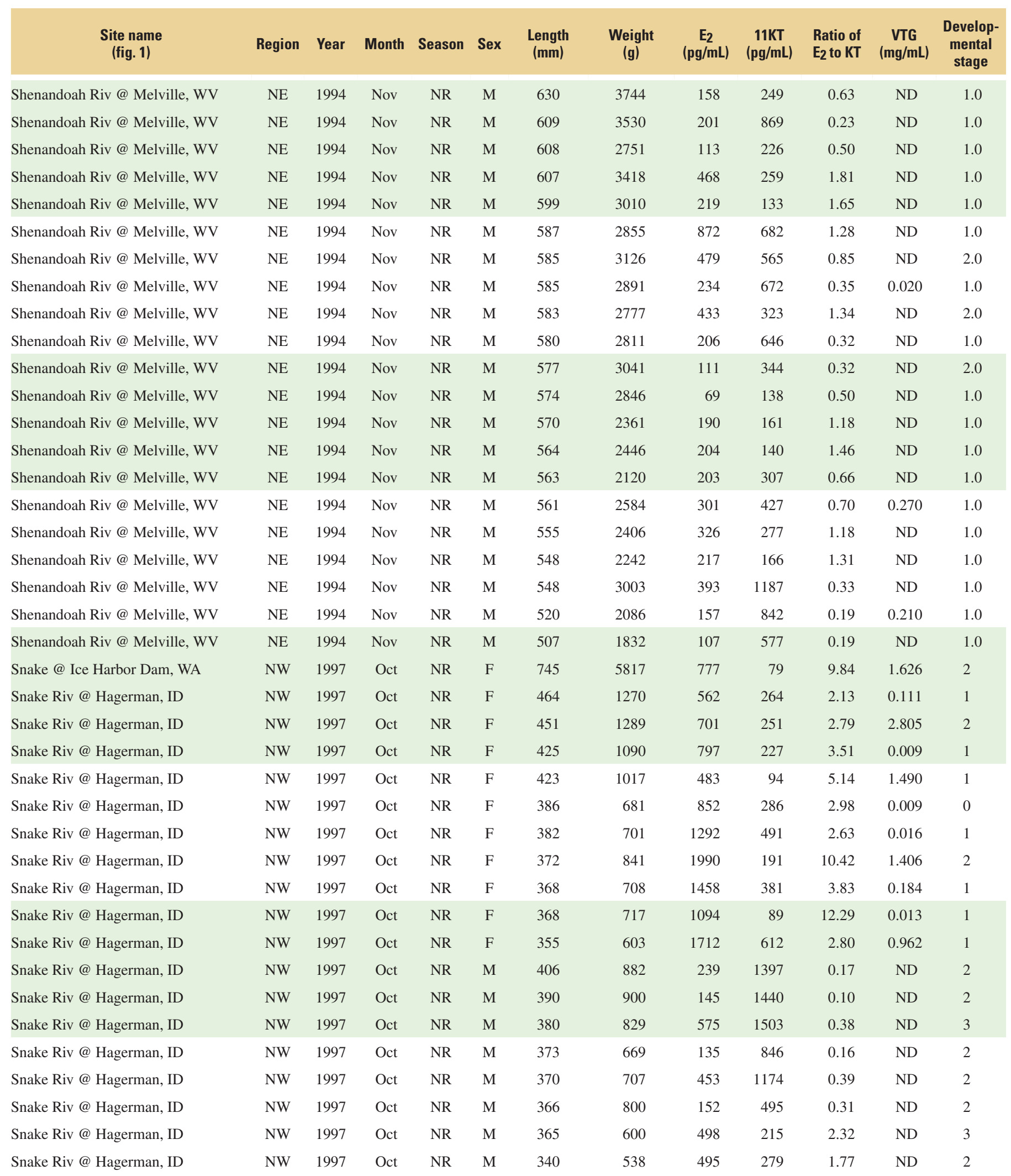


Table 4. (Continued) All individual COMMON CARP values for biomarker and ancillary data from collections made at sites in U.S. waters from August 1994 through October 1997.

[Region: NE, northeast; NW, northwest; SE, southeast; SW, southwest; NMC, northern mid-continent; SMC, southern mid-continent.

Season: NR, non-reproductive. R, reproductive. Sex: F, female, M, male. E2, 17 ß Estradiol; 11KT, 11 ketotestosterone; VTG, vitellogenin;

mm, millimeter; g, grams; pg, picograms; mL, milliliter; mg, milligram; ND, not detected; NA, not available]

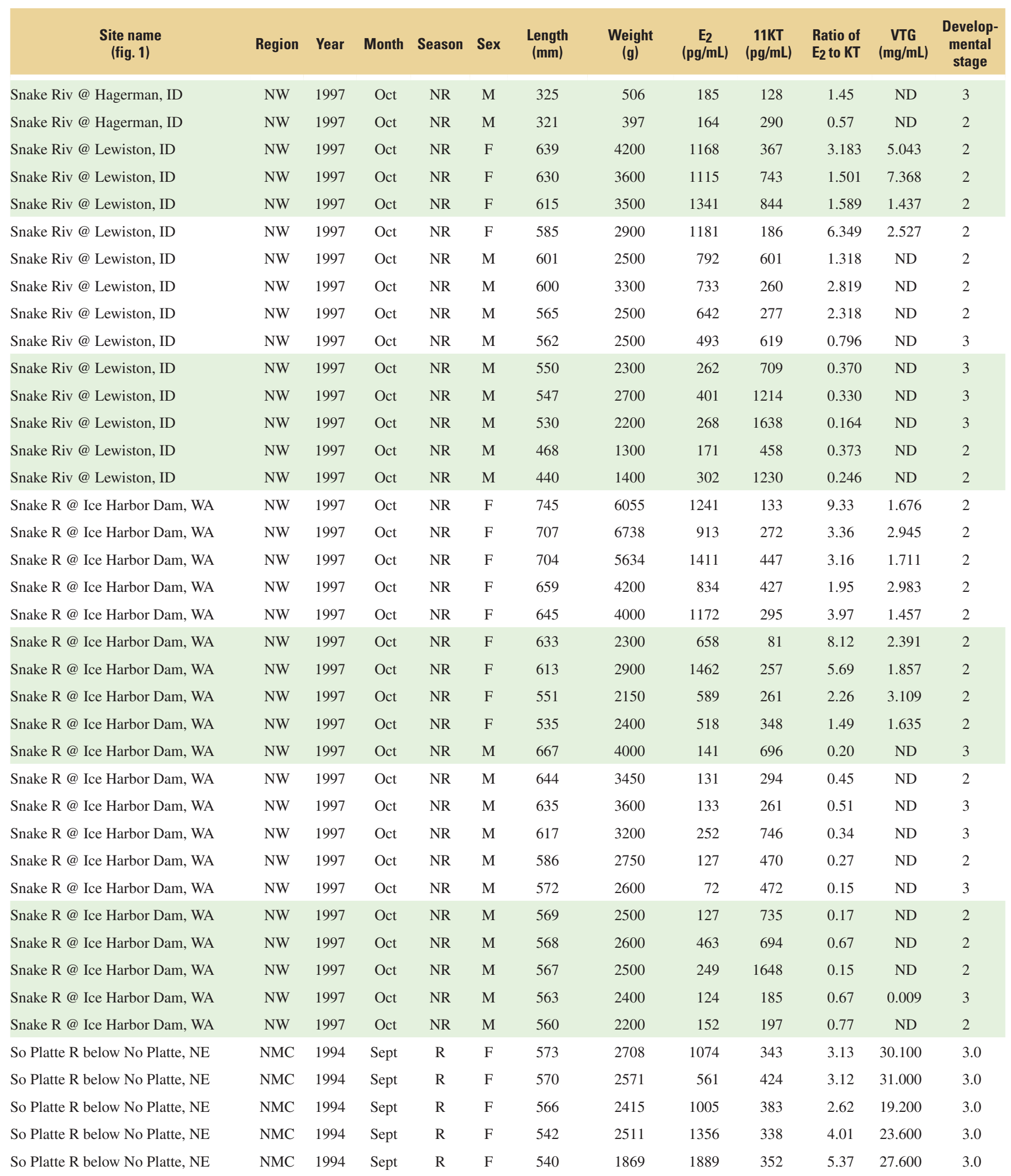


Table 4. (Continued) All individual COMMON CARP values for biomarker and ancillary data from collections made at sites in U.S. waters from August 1994 through October 1997.

[Region: NE, northeast; NW, northwest; SE, southeast; SW, southwest; NMC, northern mid-continent; SMC, southern mid-continent. Season: NR, non-reproductive. R, reproductive. Sex: F, female, M, male. E2, 17 ß Estradiol; 11KT, 11 ketotestosterone; VTG, vitellogenin; mm, millimeter; g, grams; pg, picograms; mL, milliliter; mg, milligram; ND, not detected; NA, not available]

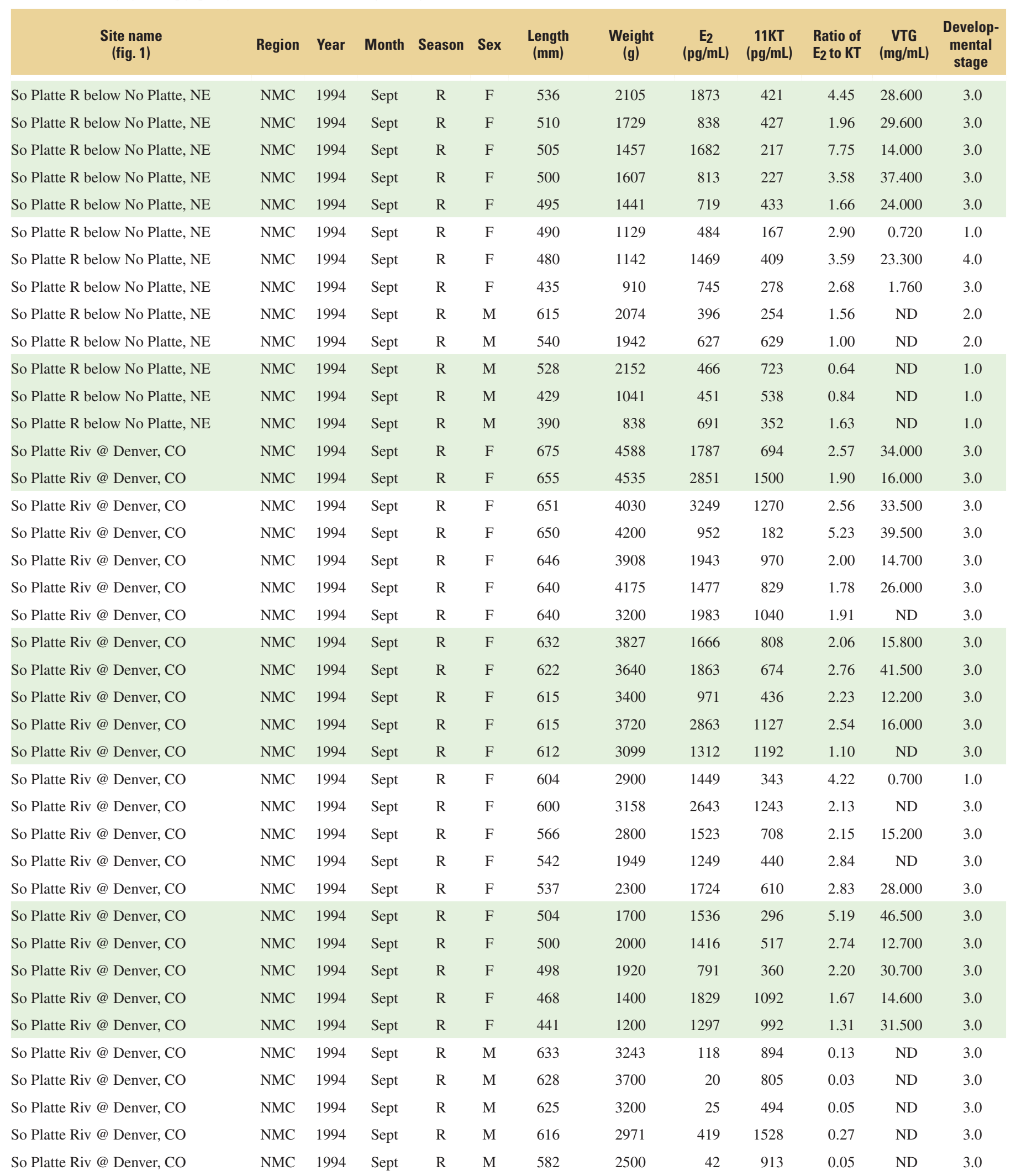


Table 4. (Continued) All individual COMMON CARP values for biomarker and ancillary data from collections made at sites in U.S. waters from August 1994 through October 1997.

[Region: NE, northeast; NW, northwest; SE, southeast; SW, southwest; NMC, northern mid-continent; SMC, southern mid-continent. Season: NR, non-reproductive. R, reproductive. Sex: F, female, M, male. E2, 17 ß Estradiol; 11KT, 11 ketotestosterone; VTG, vitellogenin; mm, millimeter; g, grams; pg, picograms; mL, milliliter; mg, milligram; ND, not detected; NA, not available]

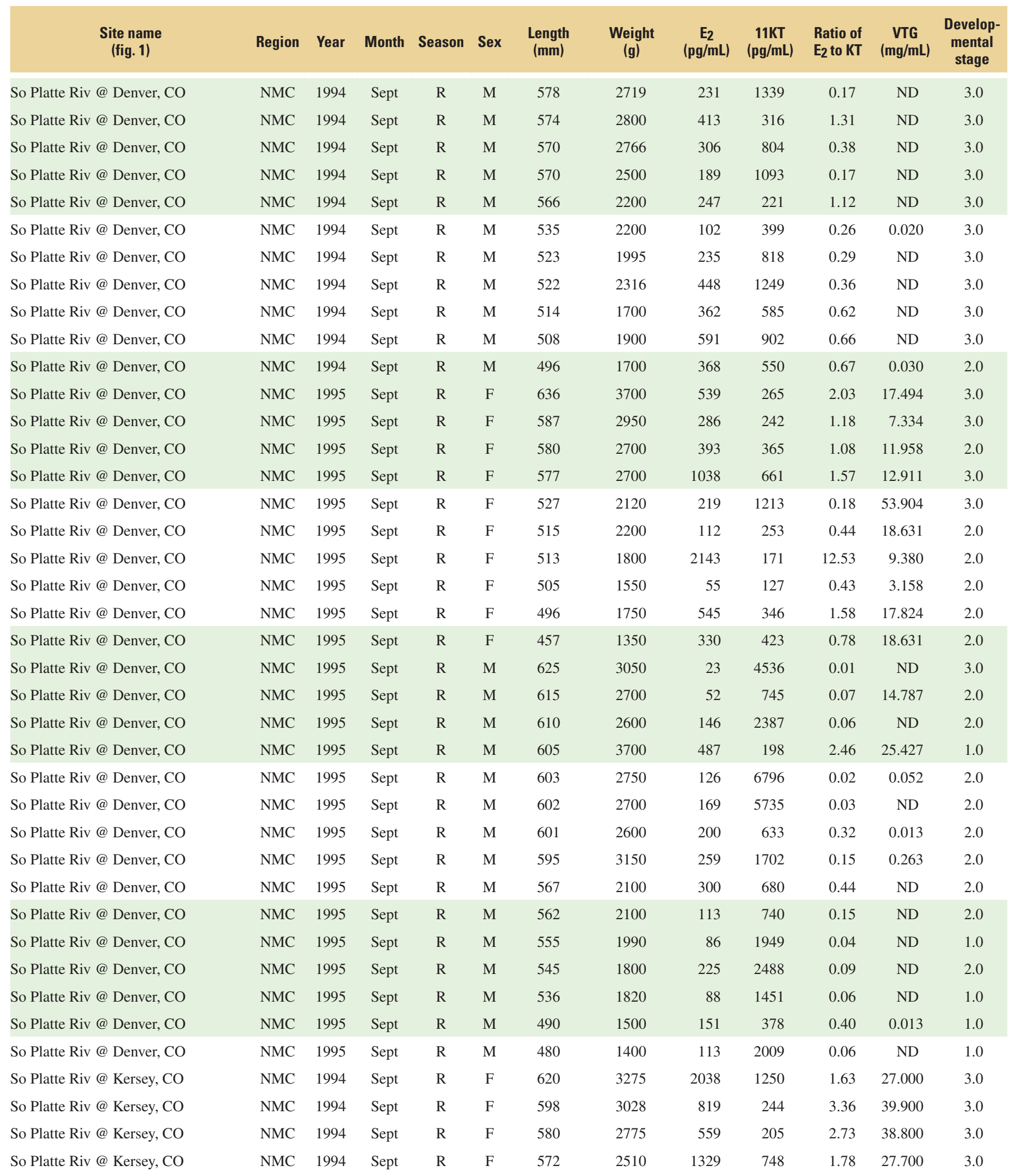


Table 4. (Continued) All individual COMMON CARP values for biomarker and ancillary data from collections made at sites in U.S. waters from August 1994 through October 1997.

[Region: NE, northeast; NW, northwest; SE, southeast; SW, southwest; NMC, northern mid-continent; SMC, southern mid-continent. Season: NR, non-reproductive. R, reproductive. Sex: F, female, M, male. E2, 17 ß Estradiol; 11KT, 11 ketotestosterone; VTG, vitellogenin; mm, millimeter; g, grams; pg, picograms; mL, milliliter; mg, milligram; ND, not detected; NA, not available]

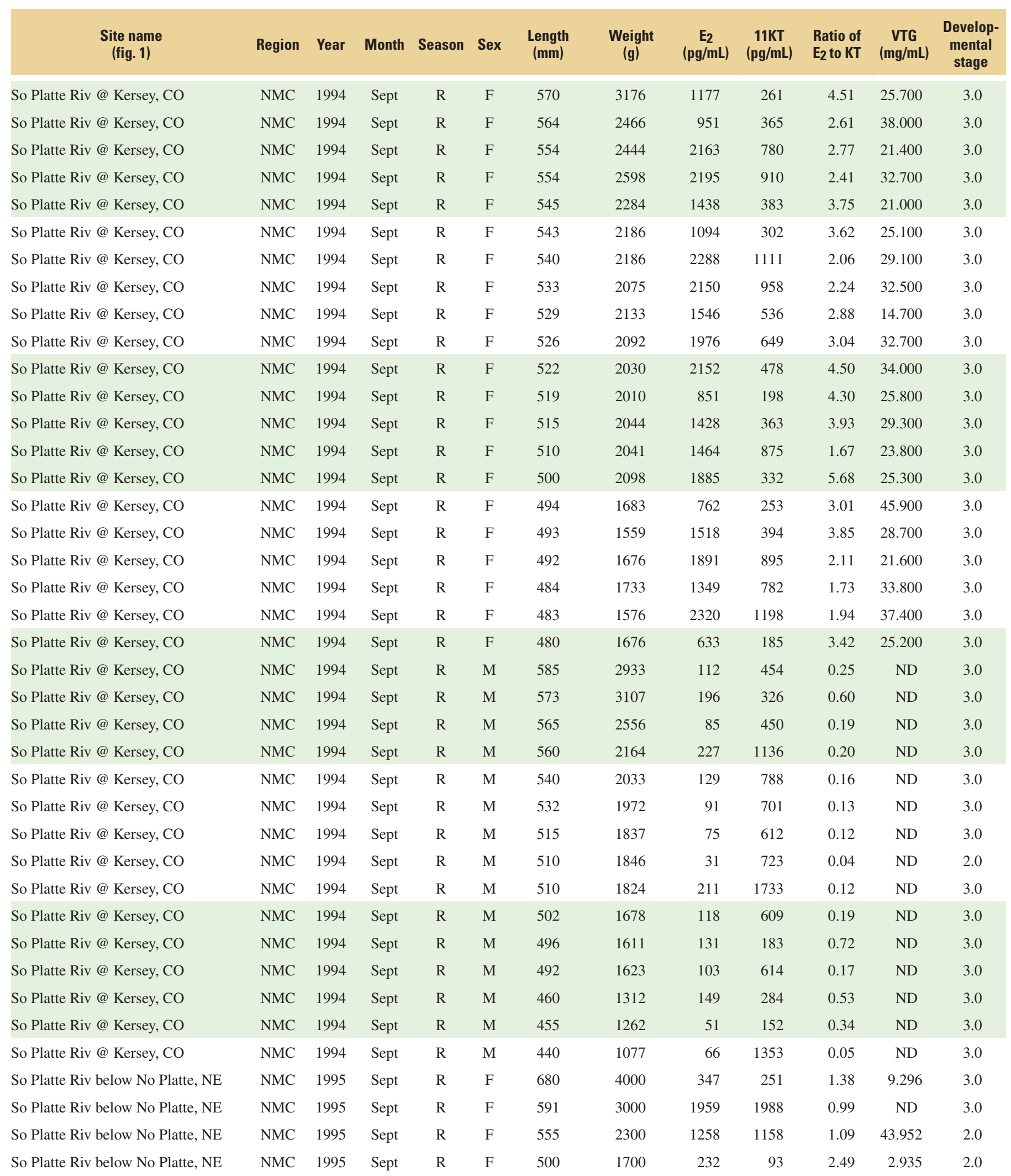


Table 4. (Continued) All individual COMMON CARP values for biomarker and ancillary data from collections made at sites in U.S. waters from August 1994 through October 1997.

[Region: NE, northeast; NW, northwest; SE, southeast; SW, southwest; NMC, northern mid-continent; SMC, southern mid-continent.

Season: NR, non-reproductive. R, reproductive. Sex: F, female, M, male. E2, 17 ß Estradiol; 11KT, 11 ketotestosterone; VTG, vitellogenin; mm, millimeter; g, grams; pg, picograms; mL, milliliter; mg, milligram; ND, not detected; NA, not available]

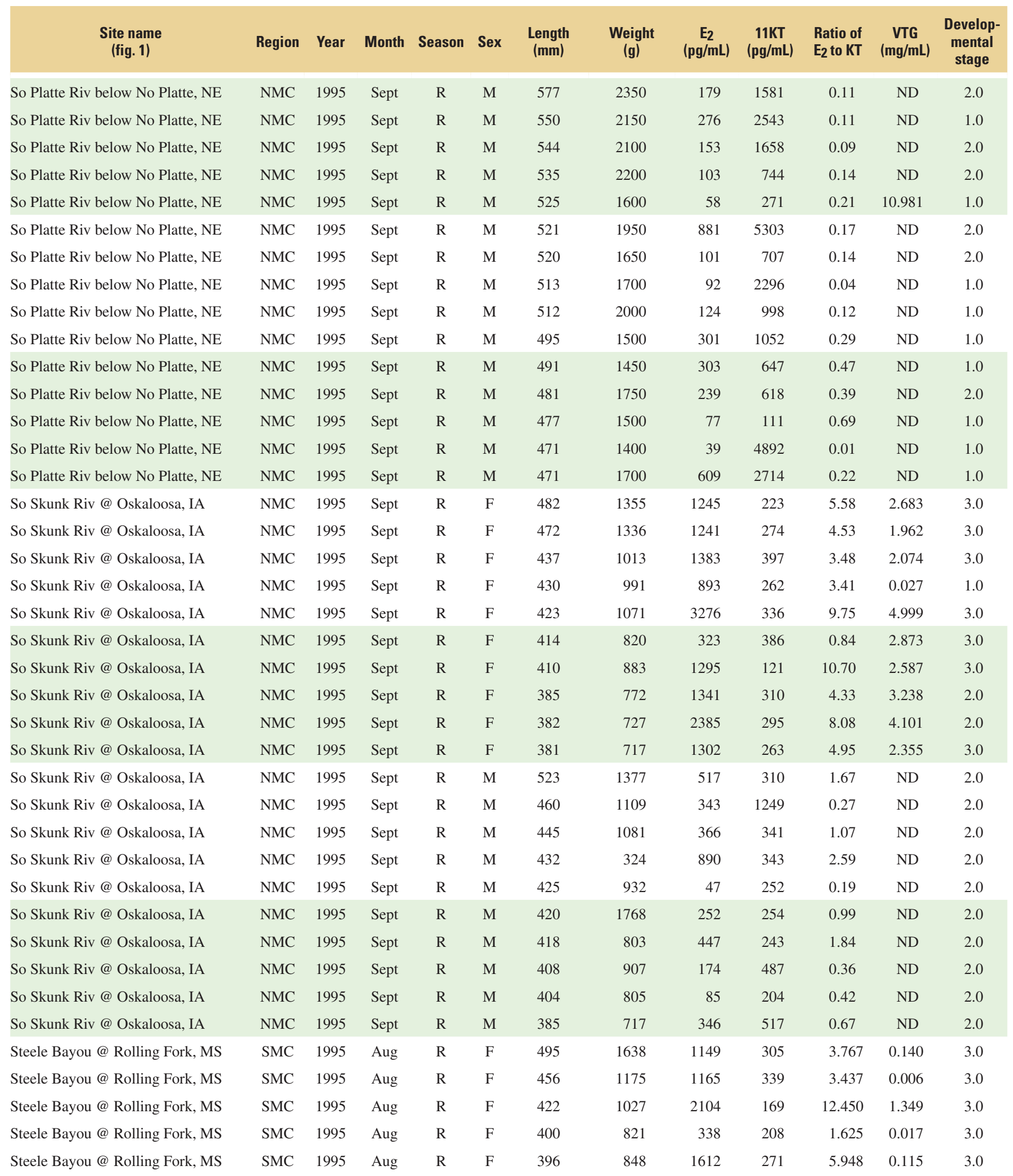


Table 4. (Continued) All individual COMMON CARP values for biomarker and ancillary data from collections made at sites in U.S. waters from August 1994 through October 1997.

[Region: NE, northeast; NW, northwest; SE, southeast; SW, southwest; NMC, northern mid-continent; SMC, southern mid-continent. Season: NR, non-reproductive. R, reproductive. Sex: F, female, M, male. E2, 17 ß Estradiol; 11KT, 11 ketotestosterone; VTG, vitellogenin; mm, millimeter; g, grams; pg, picograms; mL, milliliter; mg, milligram; ND, not detected; NA, not available]

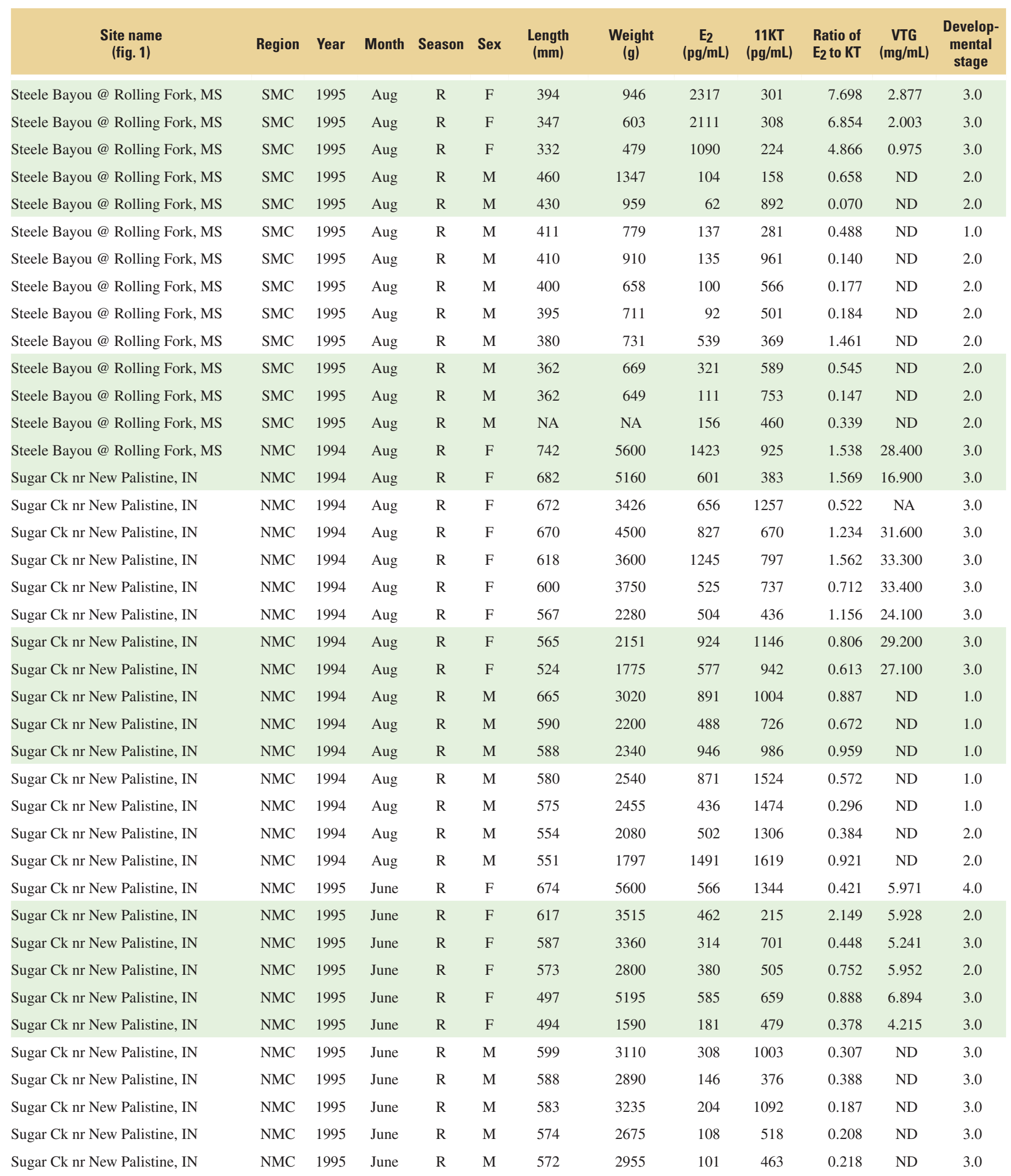


Table 4. (Continued) All individual COMMON CARP values for biomarker and ancillary data from collections made at sites in U.S. waters from August 1994 through October 1997.

[Region: NE, northeast; NW, northwest; SE, southeast; SW, southwest; NMC, northern mid-continent; SMC, southern mid-continent.

Season: NR, non-reproductive. R, reproductive. Sex: F, female, M, male. E2, 17 ß Estradiol; 11KT, 11 ketotestosterone; VTG, vitellogenin; $\mathrm{mm}$, millimeter; g, grams; pg, picograms; mL, milliliter; mg, milligram; ND, not detected; NA, not available]

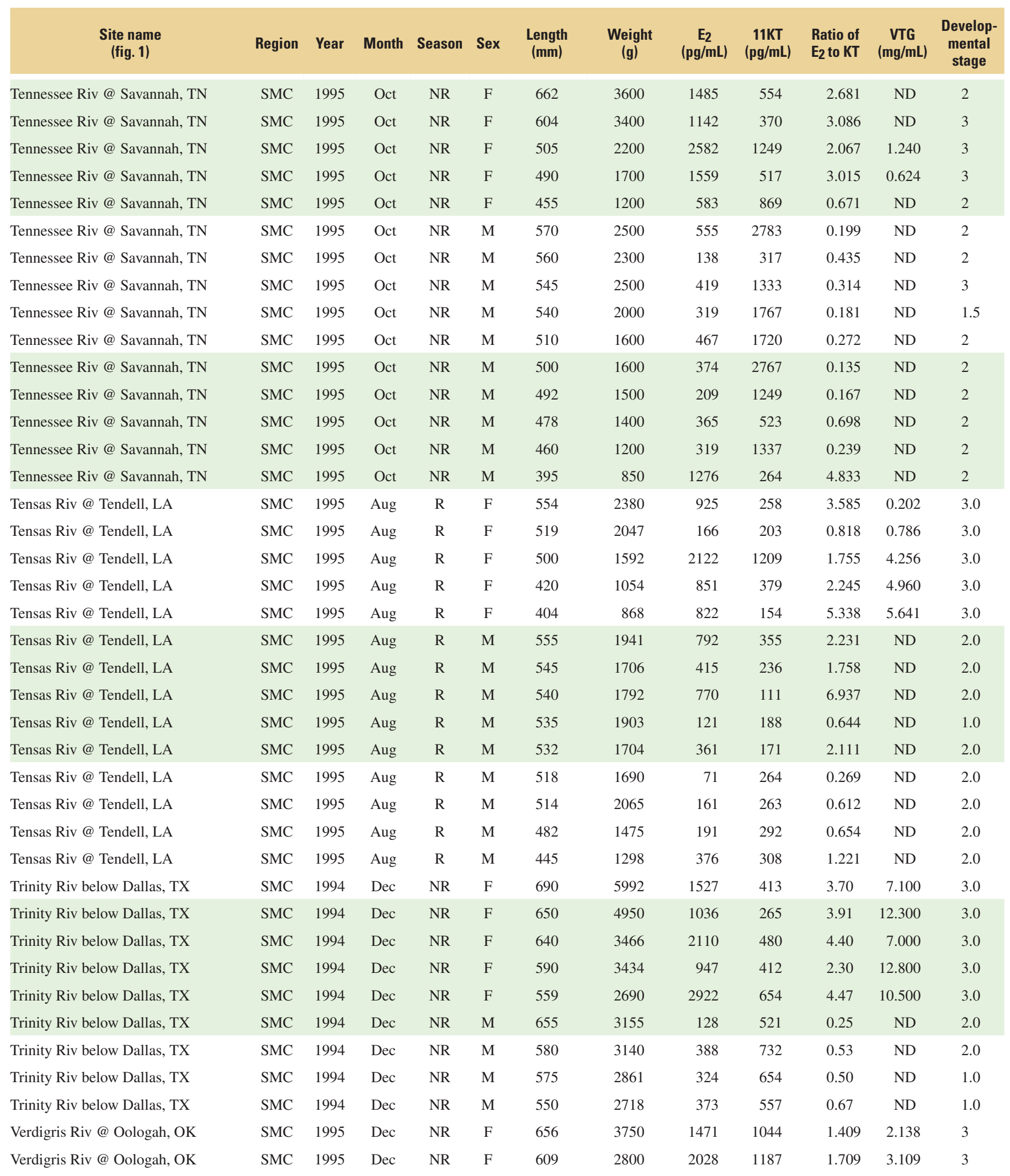


Table 4. (Continued) All individual COMMON CARP values for biomarker and ancillary data from collections made at sites in U.S. waters from August 1994 through October 1997.

[Region: NE, northeast; NW, northwest; SE, southeast; SW, southwest; NMC, northern mid-continent; SMC, southern mid-continent.

Season: NR, non-reproductive. R, reproductive. Sex: F, female, M, male. E2, 17 ß Estradiol; 11KT, 11 ketotestosterone; VTG, vitellogenin;

mm, millimeter; g, grams; pg, picograms; mL, milliliter; mg, milligram; ND, not detected; NA, not available]

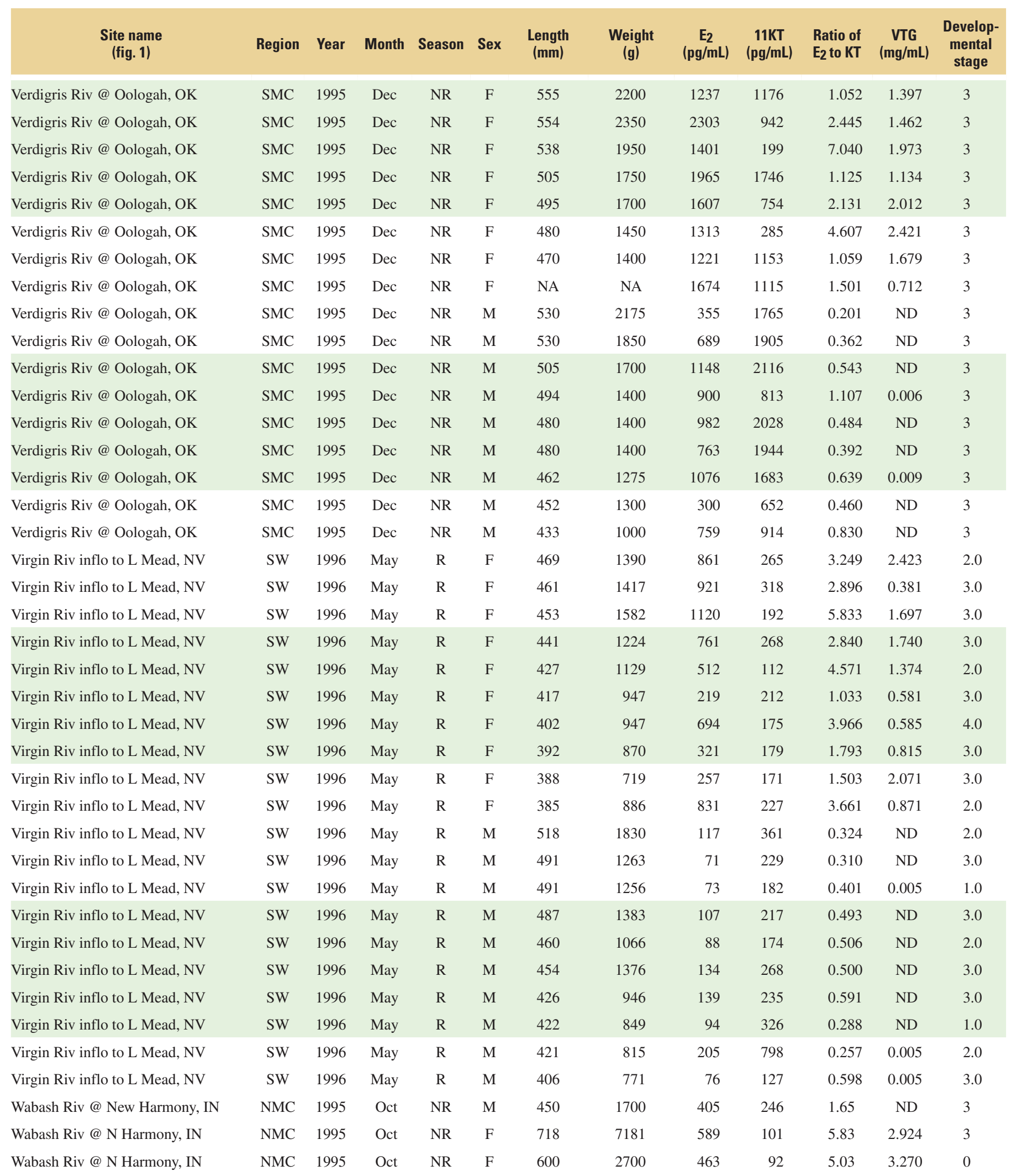


Table 4. (Continued) All individual COMMON CARP values for biomarker and ancillary data from collections made at sites in U.S. waters from August 1994 through October 1997.

[Region: NE, northeast; NW, northwest; SE, southeast; SW, southwest; NMC, northern mid-continent; SMC, southern mid-continent.

Season: NR, non-reproductive. R, reproductive. Sex: F, female, M, male. E2, 17 ß Estradiol; 11KT, 11 ketotestosterone; VTG, vitellogenin; mm, millimeter; g, grams; pg, picograms; mL, milliliter; mg, milligram; ND, not detected; NA, not available]

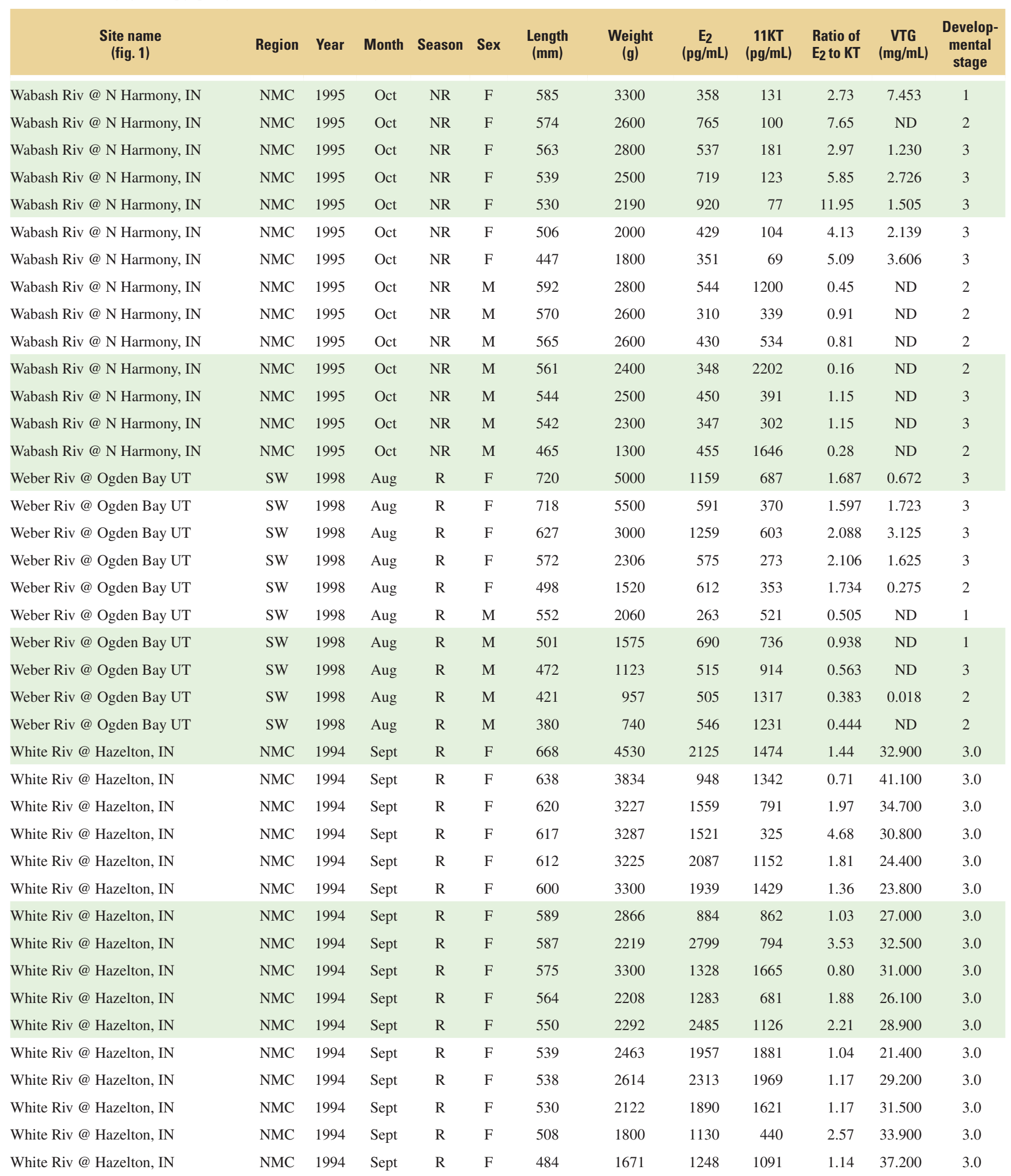


Table 4. (Continued) All individual COMMON CARP values for biomarker and ancillary data from collections made at sites in U.S. waters from August 1994 through October 1997.

[Region: NE, northeast; NW, northwest; SE, southeast; SW, southwest; NMC, northern mid-continent; SMC, southern mid-continent.

Season: NR, non-reproductive. R, reproductive. Sex: F, female, M, male. E2, 17 ß Estradiol; 11KT, 11 ketotestosterone; VTG, vitellogenin;

mm, millimeter; g, grams; pg, picograms; mL, milliliter; mg, milligram; ND, not detected; NA, not available]

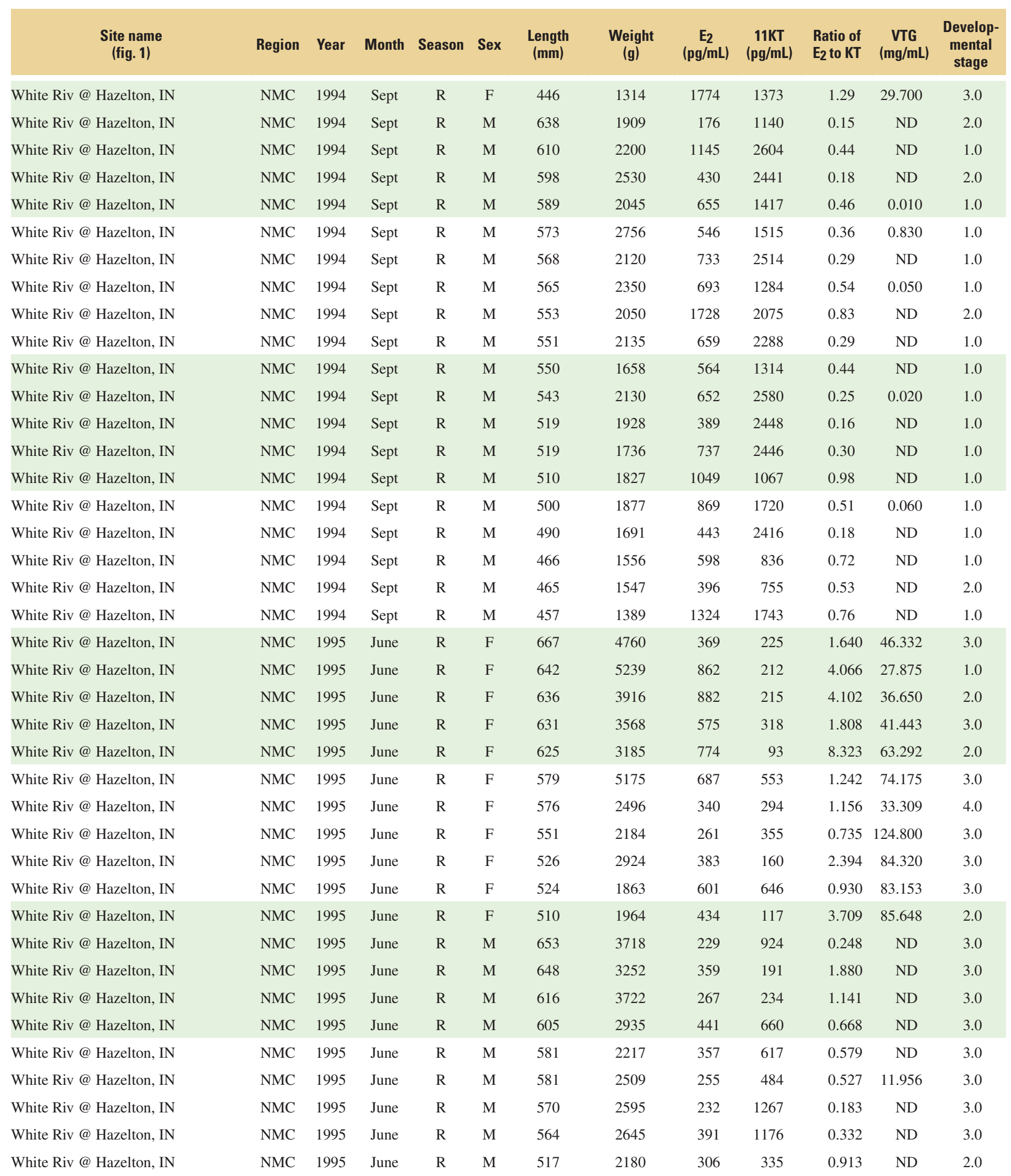


Table 4. (Continued) All individual COMMON CARP values for biomarker and ancillary data from collections made at sites in U.S. waters from August 1994 through October 1997.

[Region: NE, northeast; NW, northwest; SE, southeast; SW, southwest; NMC, northern mid-continent; SMC, southern mid-continent.

Season: NR, non-reproductive. R, reproductive. Sex: F, female, M, male. E2, 17 ß Estradiol; 11KT, 11 ketotestosterone; VTG, vitellogenin; mm, millimeter; g, grams; pg, picograms; mL, milliliter; mg, milligram; ND, not detected; NA, not available]

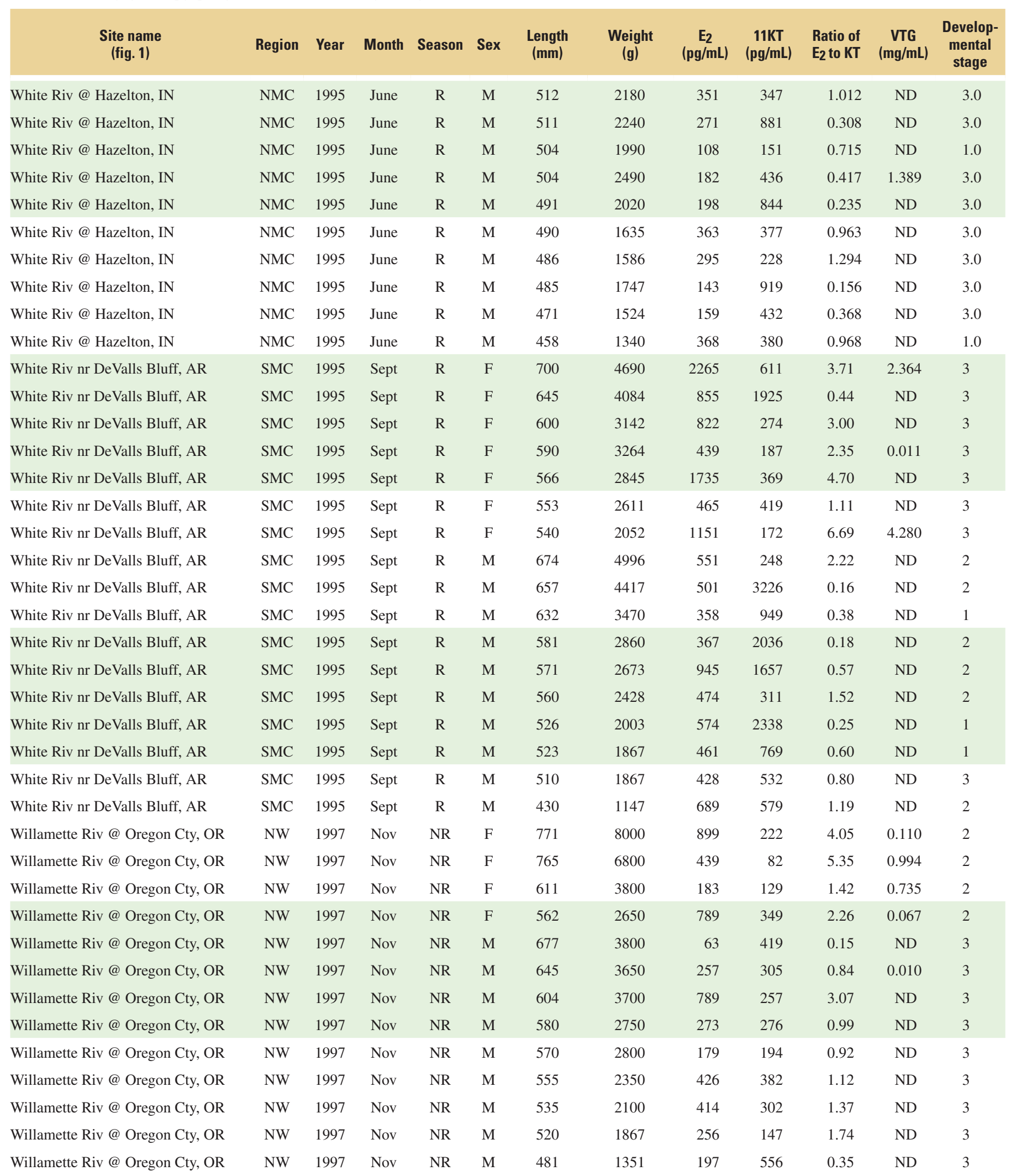


Table 4. (Continued) All individual COMMON CARP values for biomarker and ancillary data from collections made at sites in U.S. waters from August 1994 through October 1997.

[Region: NE, northeast; NW, northwest; SE, southeast; SW, southwest; NMC, northern mid-continent; SMC, southern mid-continent.

Season: NR, non-reproductive. R, reproductive. Sex: F, female, M, male. E2, 17 ß Estradiol; 11KT, 11 ketotestosterone; VTG, vitellogenin;

mm, millimeter; g, grams; pg, picograms; mL, milliliter; mg, milligram; ND, not detected; NA, not available]

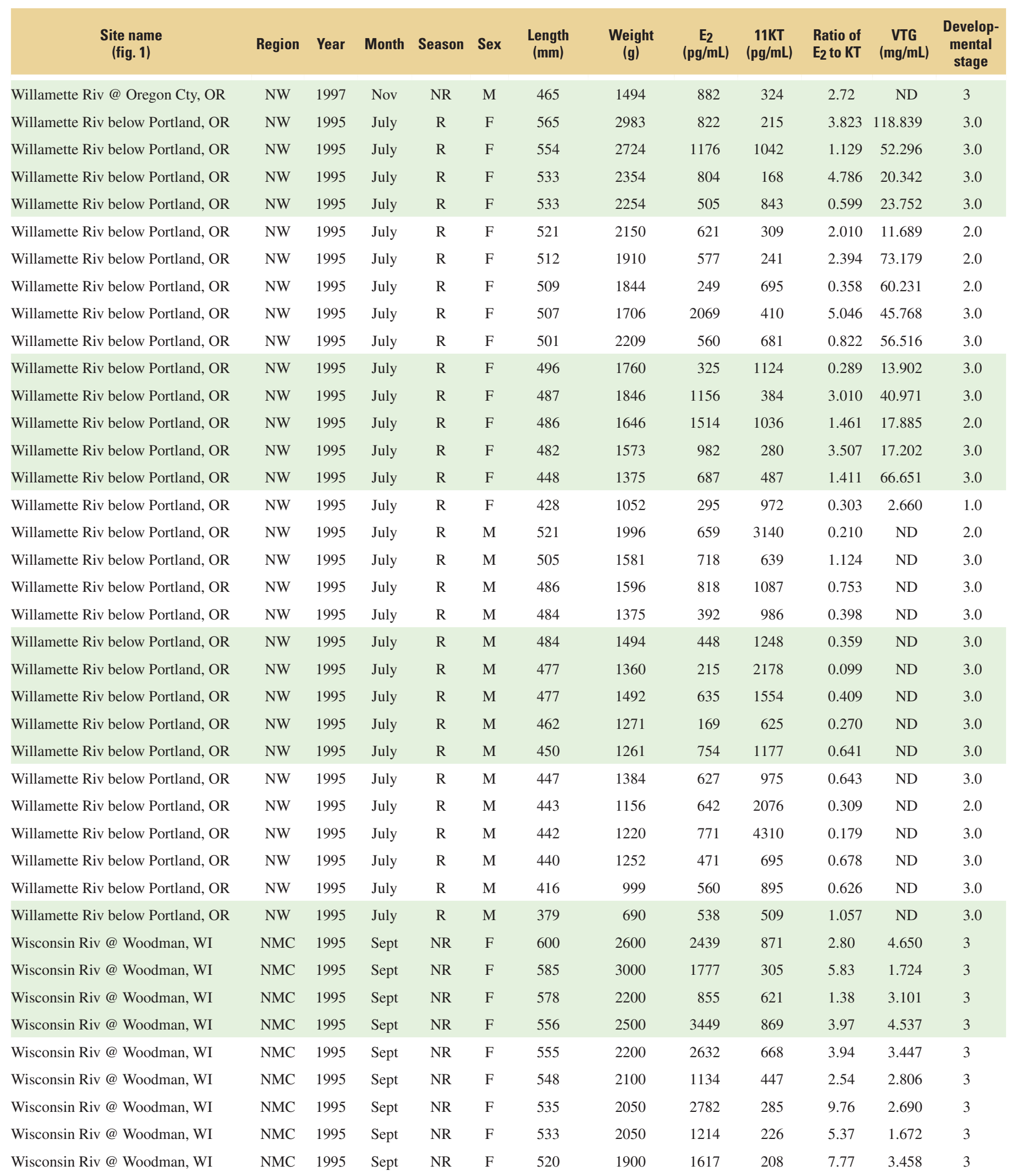


Table 4. (Continued) All individual COMMON CARP values for biomarker and ancillary data from collections made at sites in U.S. waters from August 1994 through October 1997.

[Region: NE, northeast; NW, northwest; SE, southeast; SW, southwest; NMC, northern mid-continent; SMC, southern mid-continent.

Season: NR, non-reproductive. R, reproductive. Sex: F, female, M, male. E2, 17 ß Estradiol; 11KT, 11 ketotestosterone; VTG, vitellogenin; mm, millimeter; g, grams; pg, picograms; mL, milliliter; mg, milligram; ND, not detected; NA, not available]

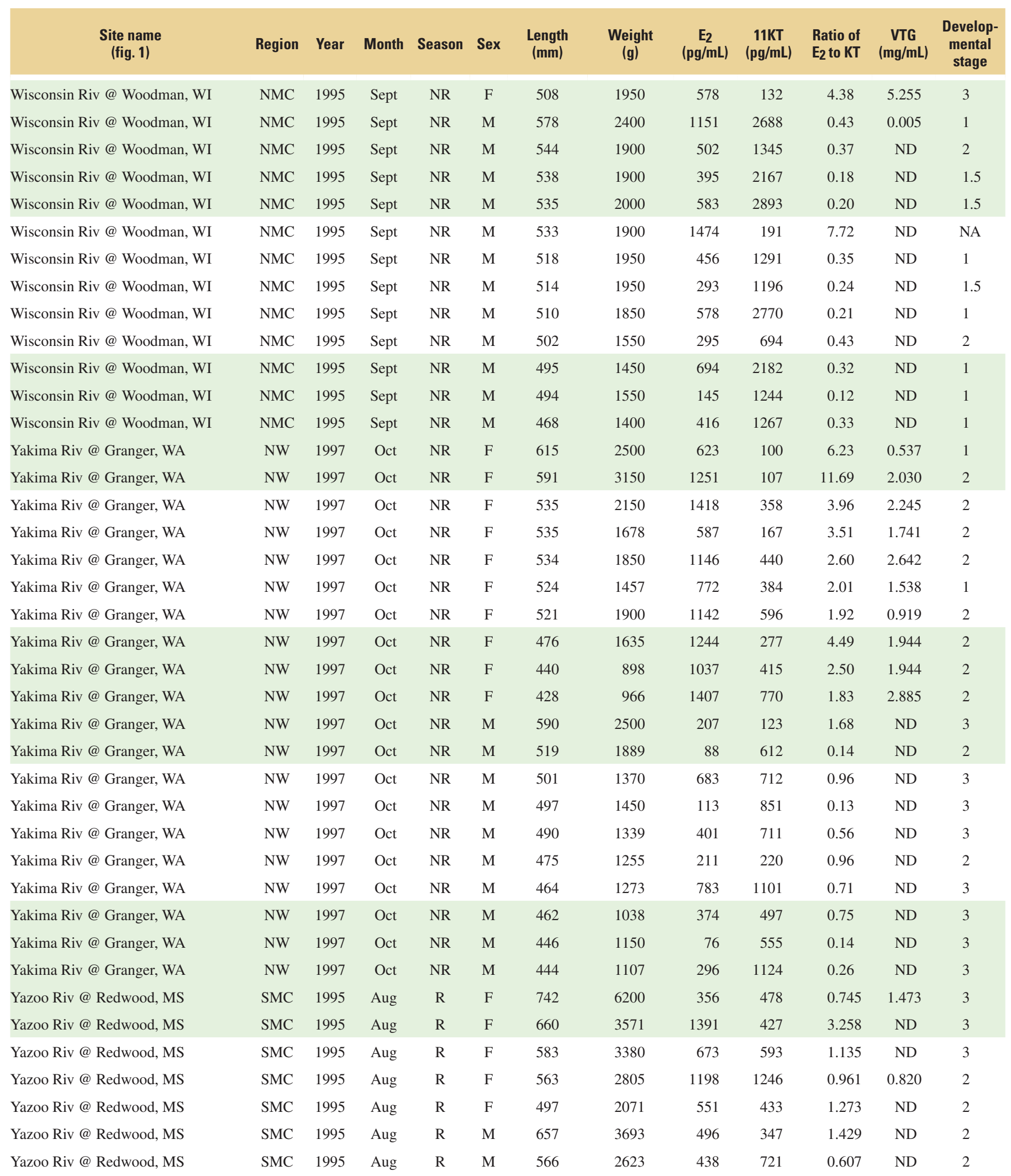


Table 4. (Continued) All individual COMMON CARP values for biomarker and ancillary data from collections made at sites in U.S. waters from August 1994 through October 1997.

[Region: NE, northeast; NW, northwest; SE, southeast; SW, southwest; NMC, northern mid-continent; SMC, southern mid-continent.

Season: NR, non-reproductive. R, reproductive. Sex: F, female, M, male. E2, 17 ß Estradiol; 11KT, 11 ketotestosterone; VTG, vitellogenin; mm, millimeter; g, grams; pg, picograms; mL, milliliter; mg, milligram; ND, not detected; NA, not available]

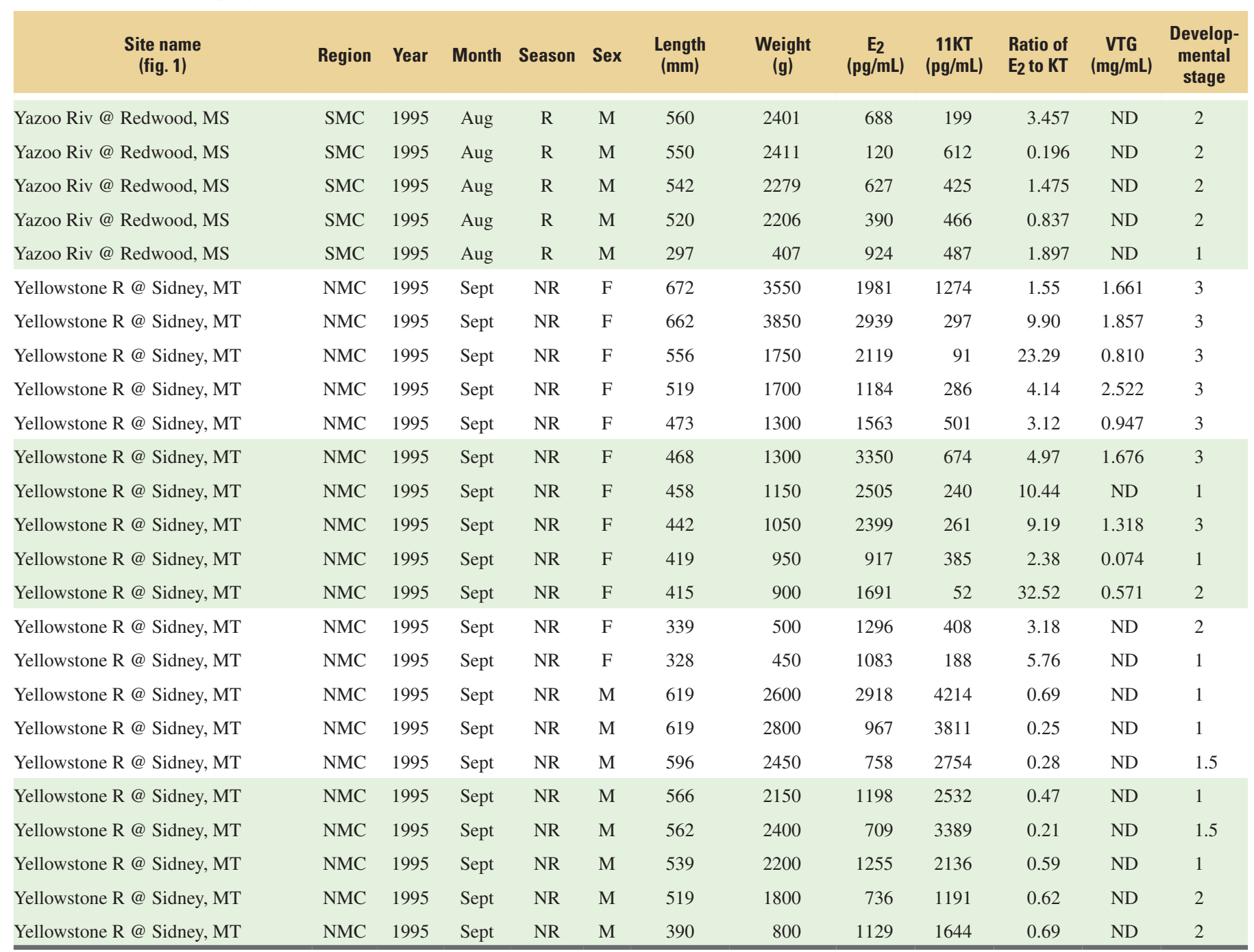


Table 5. All individual LARGEMOUTH BASS values for biomarker and ancillary data from collections made at sites in U.S. waters from March 1994 through August 1997.

[Region: NE, northeast; NW, northwest; SE, southeast; SW, southwest; NMC, northern mid-continent; SMC, southern mid-continent. Season: NR, non-reproductive. R, reproductive. Sex: F, female, M, male. E2, 17 B Estradiol; 11KT, 11 ketotestosterone; VTG, vitellogenin; mm, millimeter; g, grams; pg, picograms; mL, milliliter; mg, milligram; ND, not detected; NA, not available]

\begin{tabular}{|c|c|c|c|c|c|c|c|c|c|c|c|c|}
\hline $\begin{array}{l}\text { Site name } \\
\text { (fig. 1) }\end{array}$ & Region & Year & Month & Season & Sex & $\begin{array}{c}\text { Length } \\
\text { (mm) }\end{array}$ & $\begin{array}{c}\text { Weight } \\
\text { (g) }\end{array}$ & $\underset{(p g / m L)}{E_{2}}$ & $\begin{array}{c}11 \mathrm{KT} \\
(\mathrm{pg} / \mathrm{mL})\end{array}$ & $\begin{array}{l}\text { Ratio of } \\
E_{2} \text { to KT }\end{array}$ & $\underset{(\mathrm{mg} / \mathrm{mL})}{\text { VTG }}$ & $\begin{array}{c}\text { Develop- } \\
\text { mental } \\
\text { stage }\end{array}$ \\
\hline Arkansas Riv@ Keystone Res, OK & SMC & 1995 & Dec & NR & M & 400 & 1000 & 427 & 1563 & 0.2732 & ND & 2 \\
\hline Arkansas Riv @ Keystone Res, OK & SMC & 1995 & Dec & NR & M & 380 & 800 & 396 & 661 & 0.5991 & ND & 2 \\
\hline Arkansas Riv @ Keystone Res, OK & SMC & 1995 & Dec & NR & M & 354 & 700 & 402 & 1365 & 0.2945 & ND & 2 \\
\hline Arkansas Riv@ Keystone Res, OK & $\mathrm{SMC}$ & 1995 & Dec & NR & M & 357 & 650 & 281 & 1313 & 0.2140 & ND & 3 \\
\hline Arkansas Riv@ Keystone Res, OK & $\mathrm{SMC}$ & 1995 & Dec & NR & M & 378 & 700 & 422 & 755 & 0.5589 & 0.01 & 2 \\
\hline Arkansas Riv@ Keystone Res, OK & $\mathrm{SMC}$ & 1995 & Dec & NR & $\mathrm{F}$ & 332 & 500 & 1094 & 149 & 7.3423 & ND & 3 \\
\hline Arkansas Riv @ Keystone Res, OK & SMC & 1995 & Dec & NR & $\mathrm{F}$ & 378 & 900 & 2396 & 513 & 4.6706 & 2.3 & 3 \\
\hline Arkansas Riv @ Keystone Res, OK & $\mathrm{SMC}$ & 1995 & Dec & NR & $\mathrm{F}$ & 421 & 1400 & 705 & 114 & 6.1842 & 4.697 & 3 \\
\hline Arkansas Riv@ Keystone Res, OK & SMC & 1995 & Dec & NR & F & 378 & 825 & 978 & 121 & 8.0826 & 5.443 & 3 \\
\hline Arkansas Riv@ J Martin Res, CO & NMC & 1995 & Oct & NR & $\mathrm{F}$ & 335 & 750 & 1432 & 488 & 2.9344 & ND & 2 \\
\hline Arkansas Riv@ J Martin Res, CO & NMC & 1995 & Oct & NR & $\mathrm{F}$ & 309 & 600 & 1453 & 460 & 3.1587 & 0.01 & 2 \\
\hline Arkansas Riv@ J Martin Res, CO & NMC & 1995 & Oct & NR & $\mathrm{F}$ & 333 & 775 & 475 & 111 & 4.2793 & 0.021 & 2 \\
\hline Arkansas Riv@ J Martin Res, CO & NMC & 1995 & Oct & NR & $\mathrm{F}$ & 344 & 850 & 403 & 179 & 2.2514 & 0.043 & 2 \\
\hline Arkansas Riv @ J Martin Res, CO & NMC & 1995 & Oct & NR & $\mathrm{F}$ & 330 & 600 & 758 & 278 & 2.7266 & 0.047 & 2 \\
\hline Arkansas Riv@ J Martin Res, CO & NMC & 1995 & Oct & NR & $\mathrm{F}$ & 323 & 800 & 899 & 213 & 4.2207 & 0.131 & 2 \\
\hline Arkansas Riv @ J Martin Res, CO & NMC & 1995 & Oct & NR & M & 332 & 800 & 329 & 766 & 0.4295 & ND & 2 \\
\hline Arkansas Riv@ J Martin Res, CO & NMC & 1995 & Oct & NR & M & 337 & 752 & 388 & 1187 & 0.3269 & ND & 2 \\
\hline Arkansas Riv @ J Martin Res, CO & NMC & 1995 & Oct & NR & $\mathrm{M}$ & 327 & 750 & 535 & 1171 & 0.4569 & ND & 2 \\
\hline Arkansas Riv@ J Martin Res, CO & NMC & 1995 & Oct & NR & M & 322 & 700 & 494 & 1368 & 0.3611 & ND & 2 \\
\hline Arkansas Riv@ J Martin Res, CO & NMC & 1995 & Oct & NR & M & 328 & 660 & 493 & 2812 & 0.1753 & ND & 2 \\
\hline Arkansas Riv@ J Martin Res, CO & NMC & 1995 & Oct & NR & M & 295 & 550 & 537 & 711 & 0.7553 & ND & 2 \\
\hline Arkansas Riv @ Pine Bluff, AR & SMC & 1995 & Oct & NR & $\mathrm{F}$ & 392 & 980 & 498 & 429 & 1.1608 & ND & 2 \\
\hline Arkansas Riv@ Pine Bluff, AR & SMC & 1995 & Oct & NR & $\mathrm{F}$ & 305 & 397 & 308 & 144 & 2.1389 & ND & 2 \\
\hline Arkansas Riv@ Pine Bluff, AR & $\mathrm{SMC}$ & 1995 & Oct & NR & $\mathrm{F}$ & 315 & 391 & 235 & 329 & 0.7143 & ND & 2 \\
\hline Arkansas Riv@ Pine Bluff, AR & SMC & 1995 & Oct & NR & $\mathrm{F}$ & 405 & 970 & 749 & 319 & 2.3480 & 0.008 & 2 \\
\hline Arkansas Riv@ Pine Bluff, AR & SMC & 1995 & Oct & NR & $\mathrm{F}$ & 370 & 794 & 719 & 330 & 2.1788 & 0.009 & 2 \\
\hline Arkansas Riv@ Pine Bluff, AR & SMC & 1995 & Oct & NR & $\mathrm{F}$ & 525 & 2321 & 470 & 213 & 2.2066 & 0.018 & 2 \\
\hline Arkansas Riv@ Pine Bluff, AR & SMC & 1995 & Oct & NR & $\mathrm{F}$ & 415 & 1156 & 845 & 205 & 4.1220 & 0.019 & 2 \\
\hline
\end{tabular}


Table 5. (Continued) All individual LARGEMOUTH BASS values for biomarker and ancillary data from collections made at sites in U.S. waters from March 1994 through August 1997.

[Region: NE, northeast; NW, northwest; SE, southeast; SW, southwest; NMC, northern mid-continent; SMC, southern mid-continent.

Season: NR, non-reproductive. R, reproductive. Sex: F, female, M, male. E2, 17 ß Estradiol; 11KT, 11 ketotestosterone; VTG, vitellogenin; mm, millimeter; g, grams; pg, picograms; mL, milliliter; mg, milligram; ND, not detected; NA, not available]

\begin{tabular}{|c|c|c|c|c|c|c|c|c|c|c|c|c|}
\hline $\begin{array}{l}\text { Site name } \\
\text { (fig. 1) }\end{array}$ & Region & Year & Month & Season & Sex & $\begin{array}{l}\text { Length } \\
(\mathrm{mm})\end{array}$ & $\begin{array}{c}\text { Weight } \\
\text { (g) }\end{array}$ & $\begin{array}{c}E_{2} \\
(\mathrm{pg} / \mathrm{mL})\end{array}$ & $\begin{array}{c}11 \mathrm{KT} \\
(\mathrm{pg} / \mathrm{mL})\end{array}$ & $\begin{array}{l}\text { Ratio of } \\
E_{2} \text { to KT }\end{array}$ & $\begin{array}{c}\text { VTG } \\
\text { (mg/mL) }\end{array}$ & $\begin{array}{c}\text { Develop- } \\
\text { mental } \\
\text { stage }\end{array}$ \\
\hline Arkansas Riv@ Pine Bluff, AR & $\mathrm{SMC}$ & 1995 & Oct & NR & $\mathrm{F}$ & 505 & 2308 & 458 & 277 & 1.6534 & 0.061 & 2 \\
\hline Arkansas Riv@ Pine Bluff, AR & SMC & 1995 & Oct & NR & $\mathrm{F}$ & 540 & 2400 & 766 & 348 & 2.2011 & 0.197 & 2 \\
\hline Arkansas Riv@ Pine Bluff, AR & SMC & 1995 & Oct & NR & $\mathrm{M}$ & 380 & 783 & 523 & 622 & 0.8408 & ND & 2 \\
\hline Arkansas Riv@ Pine Bluff, AR & SMC & 1995 & Oct & NR & M & 358 & 640 & 249 & 1447 & 0.1721 & ND & 2 \\
\hline Arkansas Riv@ Pine Bluff, AR & SMC & 1995 & Oct & NR & M & 330 & 423 & 265 & 727 & 0.3645 & ND & 2 \\
\hline Arkansas Riv@ Pine Bluff, AR & SMC & 1995 & Oct & NR & $\mathrm{M}$ & 305 & 346 & 261 & 484 & 0.5393 & ND & 2 \\
\hline Arkansas Riv@ Pine Bluff, AR & SMC & 1995 & Oct & NR & M & NA & NA & 277 & 1306 & 0.2121 & ND & 1 \\
\hline Canadian Riv@Eufaula, OK & SMC & 1995 & Nov & NR & M & 453 & 1500 & 466 & 1206 & 0.3864 & ND & 2 \\
\hline CanadianRiv@Eufaula, OK & SMC & 1995 & Nov & NR & $\mathrm{M}$ & 430 & 1300 & 842 & 1393 & 0.6045 & ND & 2 \\
\hline CanadianRiv@Eufaula,OK & SMC & 1995 & Nov & NR & M & 370 & 1250 & 469 & 252 & 1.8611 & ND & 1 \\
\hline CanadianRiv@Eufaula, OK & $\mathrm{SMC}$ & 1995 & Nov & NR & M & 410 & 1000 & 704 & 1420 & 0.4958 & ND & 2 \\
\hline Canadian Riv@Eufaula, OK & SMC & 1995 & Nov & NR & M & 410 & 950 & 745 & 909 & 0.8196 & ND & 2 \\
\hline Canadian Riv@Eufaula, OK & SMC & 1995 & Nov & NR & M & 380 & 800 & 683 & 641 & 1.0655 & ND & 3 \\
\hline Canadian Riv@Eufaula,OK & SMC & 1995 & Nov & NR & $\mathrm{M}$ & 410 & 750 & 341 & 214 & 1.5935 & ND & 2 \\
\hline CanadianRiv@Eufaula, OK & SMC & 1995 & Nov & NR & M & 357 & 550 & 574 & 619 & 0.9273 & ND & 2 \\
\hline CanadianRiv@Eufaula, OK & SMC & 1995 & Nov & NR & $\mathrm{F}$ & 361 & 800 & 518 & 87 & 5.9540 & 0.287 & 3 \\
\hline CanadianRiv@Eufaula, OK & SMC & 1995 & Nov & NR & $\mathrm{F}$ & 385 & 775 & 605 & 1999 & 0.3027 & 0.675 & 3 \\
\hline Canadian Riv@Eufaula,OK & SMC & 1995 & Nov & NR & $\mathrm{F}$ & 473 & 1800 & 918 & 904 & 1.0155 & 1.581 & 3 \\
\hline CanadianRiv@Eufaula, OK & SMC & 1995 & Nov & NR & $\mathrm{F}$ & 351 & 650 & 945 & 881 & 1.0726 & 2.804 & 3 \\
\hline CanadianRiv@Eufaula, OK & SMC & 1995 & Nov & NR & $\mathrm{F}$ & 490 & 1400 & 462 & 1045 & 0.4421 & 3.414 & 2 \\
\hline CanadianRiv@Eufaula,OK & SMC & 1995 & Nov & NR & $\mathrm{F}$ & 510 & 1900 & 1022 & 1201 & 0.8510 & 4.244 & 3 \\
\hline CanadianRiv@Eufaula, OK & SMC & 1995 & Nov & NR & $\mathrm{F}$ & 400 & 1000 & 833 & 1001 & 0.8322 & 4.276 & 3 \\
\hline CanadianRiv@Eufaula,OK & SMC & 1995 & Nov & NR & $\mathrm{F}$ & 444 & 1350 & 678 & 599 & 1.1319 & 15.897 & 3 \\
\hline Chambers L nr Croscama, TX & SMC & 1996 & Sept & NR & $\mathrm{F}$ & NA & NA & 298 & 272 & 1.0956 & ND & 0 \\
\hline Chambers L nr Croscama, TX & SMC & 1996 & Sept & NR & $\mathrm{M}$ & 388 & 336 & 209 & 439 & 0.4761 & ND & 1 \\
\hline Chambers L nr Croscama, TX & SMC & 1996 & Sept & NR & M & 245 & 267 & 226 & 433 & 0.5219 & ND & 2 \\
\hline Chambers L nr Croscama, TX & SMC & 1996 & Sept & NR & M & NA & NA & 211 & 274 & 0.7701 & ND & 3 \\
\hline Chambers L nr Croscama, TX & SMC & 1996 & Sept & NR & $\mathrm{M}$ & NA & NA & 324 & 813 & 0.3985 & ND & 3 \\
\hline
\end{tabular}


Table 5. (Continued) All individual LARGEMOUTH BASS values for biomarker and ancillary data from collections made at sites in U.S. waters from March 1994 through August 1997.

[Region: NE, northeast; NW, northwest; SE, southeast; SW, southwest; NMC, northern mid-continent; SMC, southern mid-continent.

Season: NR, non-reproductive. R, reproductive. Sex: F, female, M, male. E2, 17 B Estradiol; 11KT, 11 ketotestosterone; VTG, vitellogenin; mm, millimeter; g, grams; pg, picograms; mL, milliliter; mg, milligram; ND, not detected; NA, not available]

\begin{tabular}{|c|c|c|c|c|c|c|c|c|c|c|c|c|}
\hline $\begin{array}{l}\text { Site name } \\
\text { (fig. 1) }\end{array}$ & Region & Year & Month & Season & Sex & $\begin{array}{c}\text { Length } \\
\text { (mm) }\end{array}$ & $\begin{array}{c}\text { Weight } \\
\text { (g) }\end{array}$ & $\begin{array}{c}E_{2} \\
(p g / m L)\end{array}$ & $\begin{array}{c}11 \mathrm{KT} \\
(\mathrm{pg} / \mathrm{mL})\end{array}$ & $\begin{array}{l}\text { Ratio of } \\
E_{2} \text { to KT }\end{array}$ & $\underset{(\mathrm{mg} / \mathrm{mL})}{\text { VTG }}$ & $\begin{array}{l}\text { Develop- } \\
\text { mental } \\
\text { stage }\end{array}$ \\
\hline Chickasaw NWR, TN & $\mathrm{SMC}$ & 1996 & Jan & NR & M & 405 & 964 & 247 & 218 & 1.1330 & ND & 3 \\
\hline Chickasaw NWR, TN & SMC & 1996 & Jan & NR & M & 361 & 624 & 314 & 186 & 1.6882 & ND & 1 \\
\hline Chickasaw NWR, TN & SMC & 1996 & Jan & NR & $\mathrm{F}$ & 282 & 454 & 173 & 101 & 1.7129 & 0.005 & . \\
\hline Chickasaw NWR, TN & $\mathrm{SMC}$ & 1996 & Jan & NR & $\mathrm{F}$ & 395 & 851 & 535 & 70 & 7.6429 & 0.023 & 1 \\
\hline Chickasaw NWR, TN & SMC & 1996 & Jan & NR & $\mathrm{F}$ & 573 & 2920 & 322 & 219 & 1.4703 & 0.034 & 3 \\
\hline Chickasaw NWR, TN & $\mathrm{SMC}$ & 1996 & Jan & NR & $\mathrm{F}$ & 465 & 1616 & 90 & 79 & 1.1392 & 0.141 & 1 \\
\hline Columbia Riv@Pasco,WA & NW & 1997 & Oct & NR & $\mathrm{F}$ & 323 & 556 & 468 & 141 & 3.3191 & ND & 1 \\
\hline Columbia Riv@ Pasco, WA & NW & 1997 & Oct & NR & $\mathrm{F}$ & 364 & 744 & 1465 & 387 & 3.7855 & 0.006 & 1 \\
\hline Columbia Riv@ Pasco, WA & NW & 1997 & Oct & NR & $\mathrm{F}$ & 269 & 299 & 881 & 908 & 0.9703 & 0.155 & 1 \\
\hline Columbia Riv@ Pasco,WA & NW & 1997 & Oct & NR & $\mathrm{F}$ & 320 & 452 & 833 & 581 & 1.4337 & 0.172 & 1 \\
\hline Columbia Riv@ Pasco, WA & NW & 1997 & Oct & NR & $\mathrm{F}$ & 283 & 337 & 239 & 173 & 1.3815 & 0.172 & 1 \\
\hline Columbia Riv@ Pasco, WA & NW & 1997 & Oct & NR & $\mathrm{F}$ & 522 & 2600 & 351 & 208 & 1.6875 & 0.61 & 1 \\
\hline Columbia Riv@ Pasco, WA & NW & 1997 & Oct & NR & $\mathrm{F}$ & 407 & 1450 & 553 & 122 & 4.5328 & 33.665 & 3 \\
\hline Columbia Riv@ Pasco, WA & NW & 1997 & Oct & NR & M & 440 & 1248 & 298 & 654 & 0.4557 & ND & 2 \\
\hline Columbia Riv@ Pasco, WA & NW & 1997 & Oct & NR & M & 261 & 282 & 182 & 331 & 0.5498 & ND & 2 \\
\hline Connecticut R @ Thompsonville, CT & NE & 1994 & Sept & NR & $\mathrm{F}$ & 373 & 784 & 307 & 93 & 3.3011 & 0.008 & 1 \\
\hline Connecticut R @ Thompsonville, CT & $\mathrm{NE}$ & 1994 & Sept & NR & M & 295 & 379 & 1500 & 1203 & 1.2469 & ND & 3 \\
\hline Connecticut R @ Thompsonville, CT & $\mathrm{NE}$ & 1994 & Sept & NR & M & 284 & 314 & 718 & 1206 & 0.5954 & ND & 3 \\
\hline Connecticut R @ Thompsonville, CT & NE & 1994 & Sept & NR & M & 284 & 370 & 231 & 361 & 0.6399 & 0.0014 & 3 \\
\hline Connecticut R @ Thompsonville, CT & $\mathrm{NE}$ & 1994 & Sept & NR & M & 393 & 494 & 386 & 257 & 1.5019 & 0.002 & 3 \\
\hline Cumberland Riv@Clarksville,TN & SMC & 1995 & Oct & NR & $\mathrm{F}$ & 268 & 235 & 563 & 70 & 8.0429 & ND & 3 \\
\hline CumberlandRiv@Clarksville,TN & $\mathrm{SMC}$ & 1995 & Oct & NR & $\mathrm{F}$ & 231 & 142 & 230 & 49 & 4.6939 & ND & 2 \\
\hline CumberlandRiv@Clarksville,TN & SMC & 1995 & Oct & NR & $\mathrm{F}$ & 325 & 425 & 313 & 89 & 3.5169 & 0.156 & 3 \\
\hline Cumberland Riv@ Clarksville,TN & SMC & 1995 & Oct & NR & $\mathrm{F}$ & 320 & 379 & 341 & 52 & 6.5577 & 0.925 & 2 \\
\hline Cumberland Riv@Clarksville,TN & SMC & 1995 & Oct & NR & M & 335 & 525 & 318 & 162 & 1.9630 & ND & 2 \\
\hline CumberlandRiv@Clarksville,TN & SMC & 1995 & Oct & NR & M & 300 & 370 & 265 & 106 & 2.5 & ND & 2 \\
\hline Cumberland Riv@ Clarksville,TN & SMC & 1995 & Oct & NR & M & 256 & 250 & 594 & 121 & 4.9091 & ND & 3 \\
\hline Cumberland Riv@ Clarksville,TN & SMC & 1995 & Oct & NR & M & 230 & 167 & 214 & 198 & 1.0808 & ND & 2 \\
\hline
\end{tabular}


Table 5. (Continued) All individual LARGEMOUTH BASS values for biomarker and ancillary data from collections made at sites in U.S. waters from March 1994 through August 1997.

[Region: NE, northeast; NW, northwest; SE, southeast; SW, southwest; NMC, northern mid-continent; SMC, southern mid-continent.

Season: NR, non-reproductive. R, reproductive. Sex: F, female, M, male. E2, 17 B Estradiol; 11KT, 11 ketotestosterone; VTG, vitellogenin; mm, millimeter; g, grams; pg, picograms; mL, milliliter; mg, milligram; ND, not detected; NA, not available]

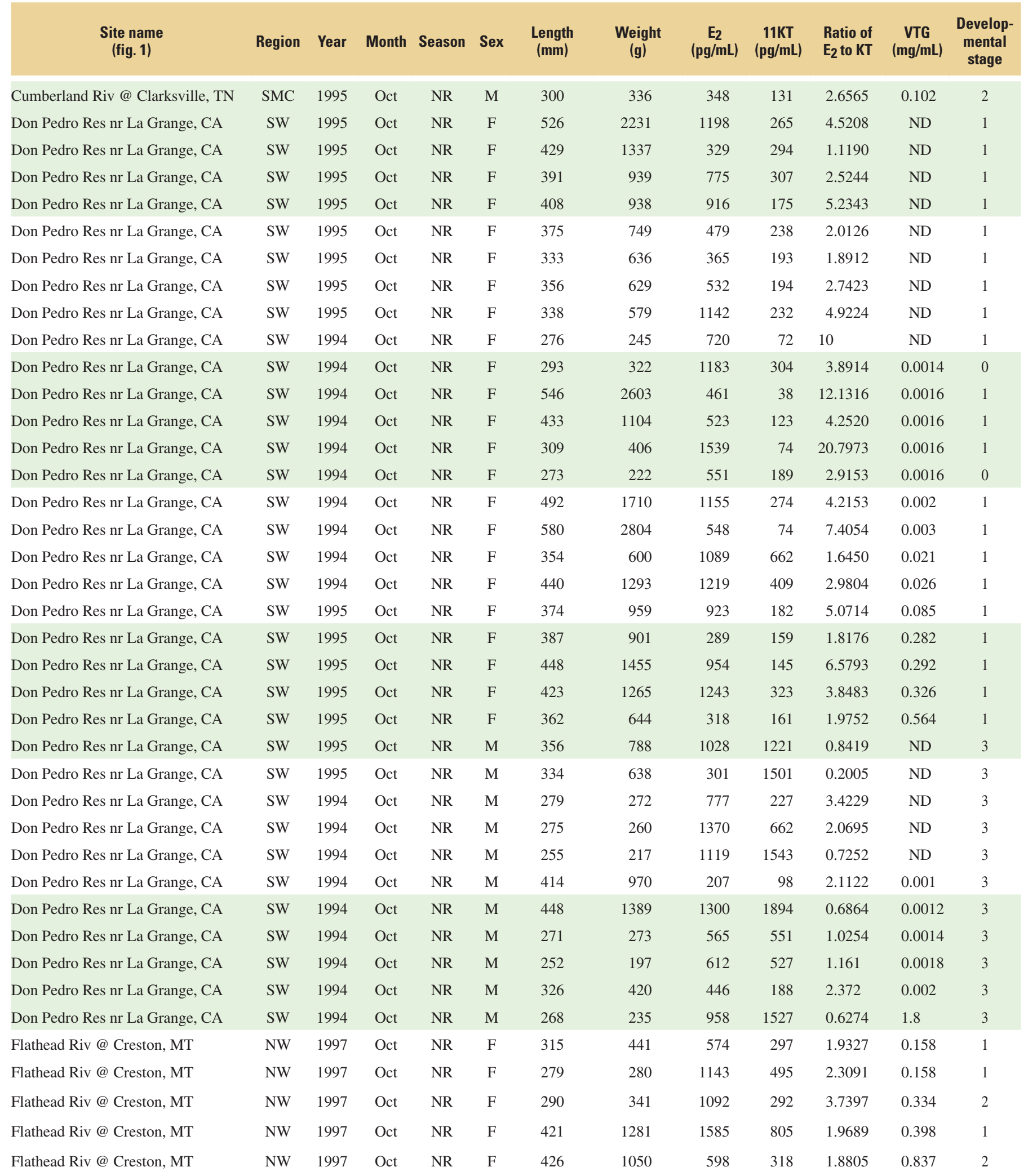


Table 5. (Continued) All individual LARGEMOUTH BASS values for biomarker and ancillary data from collections made at sites in U.S. waters from March 1994 through August 1997.

[Region: NE, northeast; NW, northwest; SE, southeast; SW, southwest; NMC, northern mid-continent; SMC, southern mid-continent.

Season: NR, non-reproductive. R, reproductive. Sex: F, female, M, male. E2, 17 B Estradiol; 11KT, 11 ketotestosterone; VTG, vitellogenin; mm, millimeter; g, grams; pg, picograms; mL, milliliter; mg, milligram; ND, not detected; NA, not available]

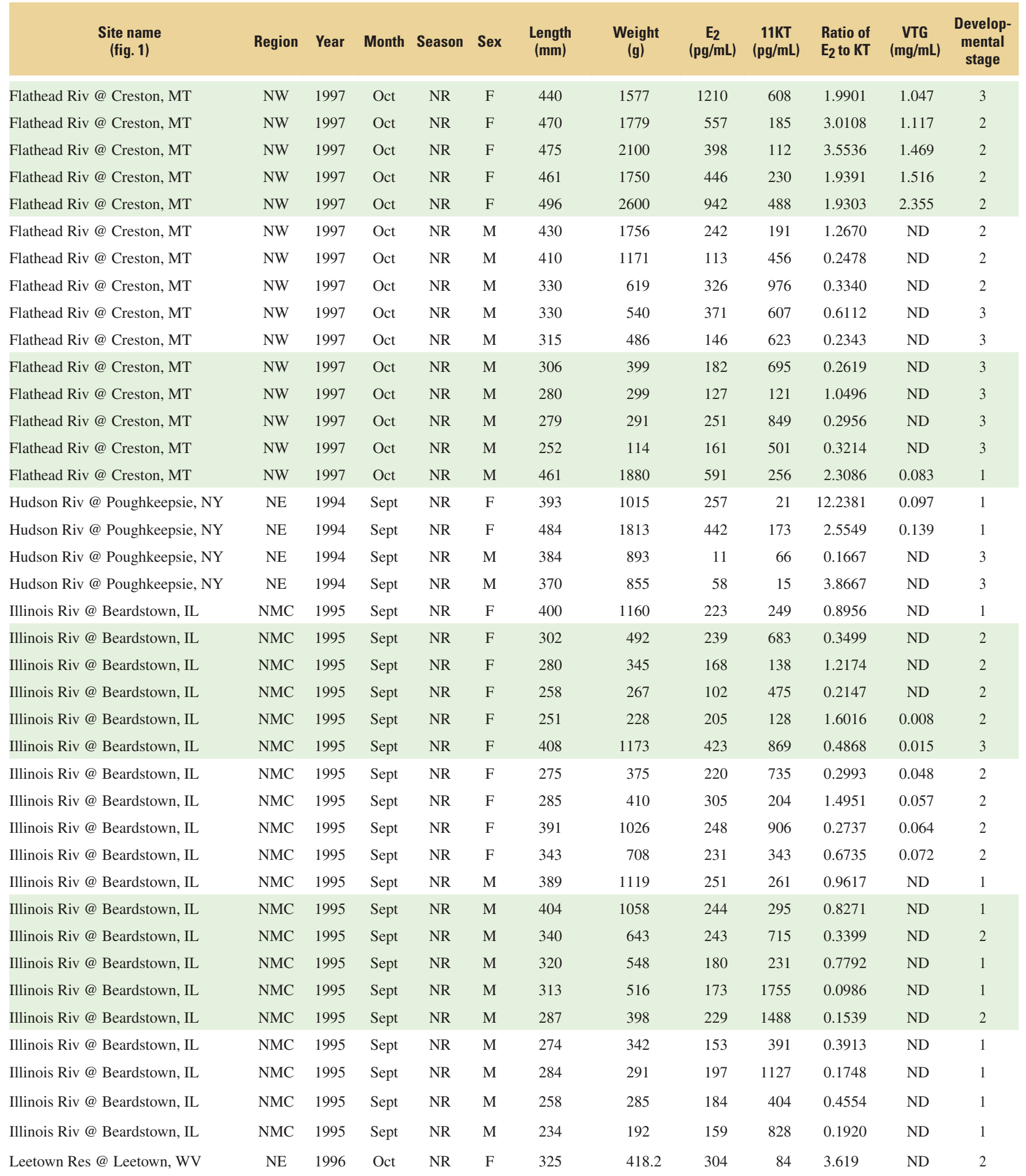


Table 5. (Continued) All individual LARGEMOUTH BASS values for biomarker and ancillary data from collections made at sites in U.S. waters from March 1994 through August 1997.

[Region: NE, northeast; NW, northwest; SE, southeast; SW, southwest; NMC, northern mid-continent; SMC, southern mid-continent.

Season: NR, non-reproductive. R, reproductive. Sex: F, female, M, male. E2, 17 ß Estradiol; 11KT, 11 ketotestosterone; VTG, vitellogenin; mm, millimeter; g, grams; pg, picograms; mL, milliliter; mg, milligram; ND, not detected; NA, not available]

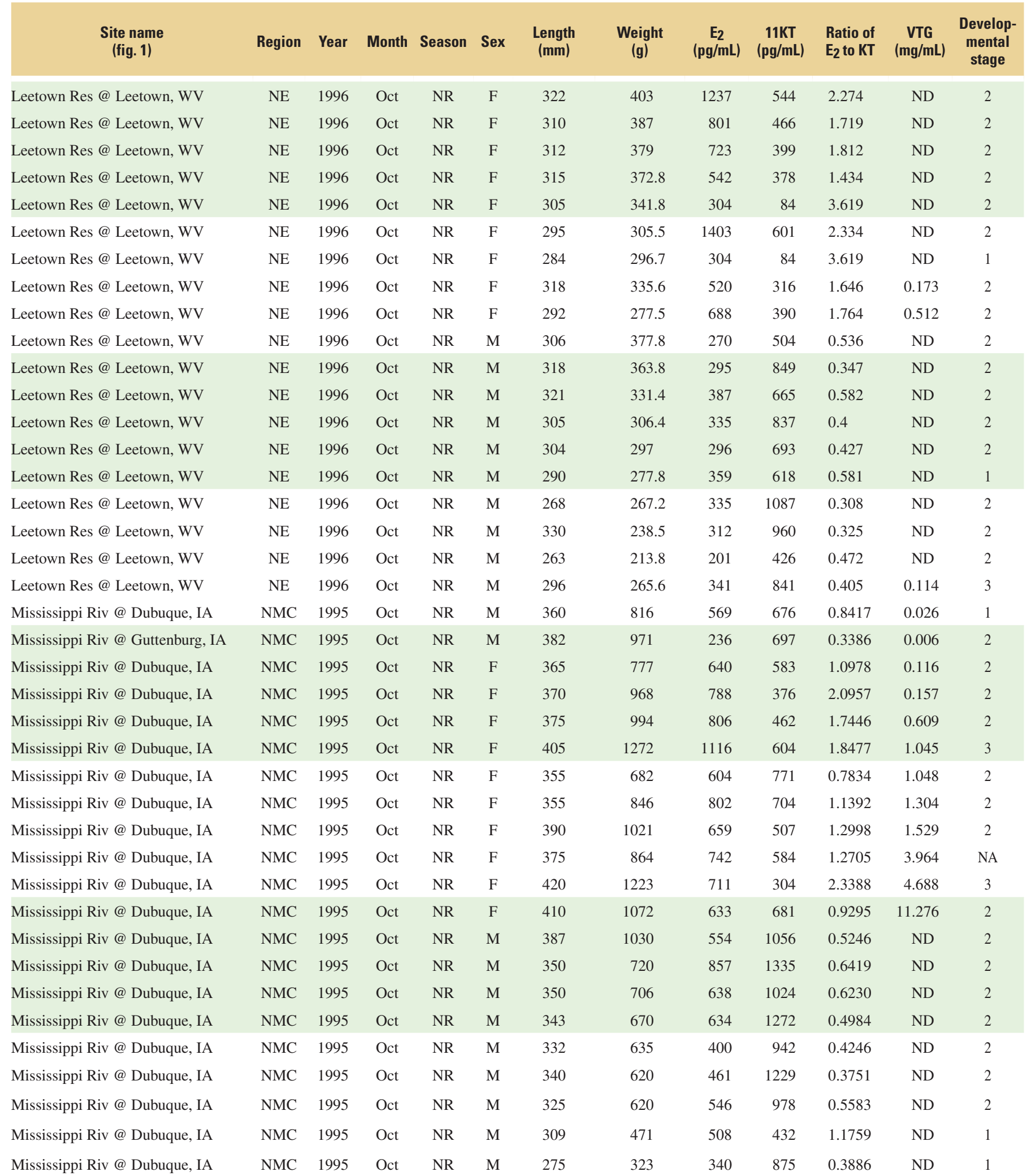


Table 5. (Continued) All individual LARGEMOUTH BASS values for biomarker and ancillary data from collections made at sites in U.S. waters from March 1994 through August 1997.

[Region: NE, northeast; NW, northwest; SE, southeast; SW, southwest; NMC, northern mid-continent; SMC, southern mid-continent.

Season: NR, non-reproductive. R, reproductive. Sex: F, female, M, male. E2, 17 B Estradiol; 11KT, 11 ketotestosterone; VTG, vitellogenin; mm, millimeter; g, grams; pg, picograms; mL, milliliter; mg, milligram; ND, not detected; NA, not available]

\begin{tabular}{|c|c|c|c|c|c|c|c|c|c|c|c|c|}
\hline $\begin{array}{l}\text { Site name } \\
\text { (fig. 1) }\end{array}$ & Region & Year & Month & Season & Sex & $\begin{array}{c}\text { Length } \\
\text { (mm) }\end{array}$ & $\begin{array}{l}\text { Weight } \\
\text { (g) }\end{array}$ & $\begin{array}{c}E_{2} \\
(p g / m L)\end{array}$ & $\begin{array}{c}11 \mathrm{KT} \\
(\mathrm{pg} / \mathrm{mL})\end{array}$ & $\begin{array}{l}\text { Ratio of } \\
E_{2} \text { to KT }\end{array}$ & $\underset{(\mathrm{mg} / \mathrm{mL})}{\text { VTG }}$ & $\begin{array}{c}\text { Develop- } \\
\text { mental } \\
\text { stage }\end{array}$ \\
\hline Mississippi Riv @ Guttenburg, IA & NMC & 1995 & Oct & NR & $\mathrm{F}$ & 425 & 1435 & 357 & 199 & 1.7940 & 0.059 & 2 \\
\hline Mississippi Riv @ Guttenburg, IA & NMC & 1995 & Oct & NR & $\mathrm{F}$ & 392 & 1016 & 1268 & 306 & 4.1438 & 0.09 & 2 \\
\hline Mississippi Riv @ Guttenburg, IA & $\mathrm{NMC}$ & 1995 & Oct & NR & $\mathrm{F}$ & 411 & 1174 & 202 & 179 & 1.1285 & 0.874 & 2 \\
\hline Mississippi Riv @ Guttenburg, IA & NMC & 1995 & Oct & NR & $\mathrm{F}$ & 423 & 1223 & 1285 & 463 & 2.7754 & 1.025 & 2 \\
\hline Mississippi Riv @ Guttenburg, IA & NMC & 1995 & Oct & NR & $\mathrm{F}$ & 390 & 1043 & 1371 & 314 & 4.3662 & 1.108 & 2 \\
\hline Mississippi Riv @ Guttenburg, IA & NMC & 1995 & Oct & NR & $\mathrm{F}$ & 390 & 940 & 1289 & 739 & 1.7442 & 1.471 & 2 \\
\hline Mississippi Riv@ Guttenburg, IA & NMC & 1995 & Oct & NR & M & 387 & 918 & 132 & 736 & 0.1793 & ND & 2 \\
\hline Mississippi Riv @ Guttenburg, IA & NMC & 1995 & Oct & NR & M & 375 & 867 & 291 & 386 & 0.7539 & ND & 2 \\
\hline Mississippi Riv@ Guttenburg, IA & NMC & 1995 & Oct & NR & M & 372 & 843 & 210 & 719 & 0.2921 & ND & 1 \\
\hline Mississippi Riv @ Guttenburg, IA & NMC & 1995 & Oct & NR & M & 365 & 783 & 289 & 675 & 0.4281 & ND & 2 \\
\hline Mississippi Riv @ Guttenburg, IA & NMC & 1995 & Oct & NR & M & 363 & 771 & 99 & 627 & 0.1579 & ND & 2 \\
\hline Mississippi Riv @ Guttenburg, IA & NMC & 1995 & Oct & NR & M & 342 & 630 & 215 & 507 & 0.4241 & ND & 2 \\
\hline Mississippi Riv @ Guttenburg, IA & NMC & 1995 & Oct & NR & M & 310 & 449 & 266 & 901 & 0.2952 & ND & 2 \\
\hline Mississippi Riv@ Memphis, TN & SMC & 1995 & Oct & NR & $\mathrm{F}$ & 301 & 328 & 196 & 132 & 1.4848 & ND & 2 \\
\hline Mississippi Riv@ Memphis, TN & SMC & 1995 & Oct & NR & F & 295 & 318 & 367 & 422 & 0.8697 & ND & 2 \\
\hline Mississippi Riv@ Memphis, TN & SMC & 1995 & Oct & NR & M & 362 & 734 & 788 & 115 & 6.8522 & ND & 2 \\
\hline Mississippi Riv@ Memphis,TN & SMC & 1995 & Oct & NR & M & 361 & 667 & 238 & 389 & 0.6118 & ND & 2 \\
\hline Mississippi Riv@ Memphis, TN & SMC & 1995 & Oct & NR & M & 315 & 463 & 727 & 182 & 3.9945 & ND & 2 \\
\hline Missouri Riv@ Hermann, MO & NMC & 1995 & Oct & NR & $\mathrm{F}$ & 426 & 1225 & 436 & 289 & 1.5087 & ND & 2 \\
\hline Missouri Riv@ Hermann, MO & NMC & 1995 & Oct & NR & $\mathrm{F}$ & 300 & 375 & 789 & 447 & 1.7651 & 0.205 & 2 \\
\hline Missouri Riv@ Hermann, MO & NMC & 1995 & Oct & NR & $\mathrm{F}$ & 366 & 725 & 338 & 309 & 1.0939 & 0.352 & 2 \\
\hline Missouri Riv@ Hermann, MO & NMC & 1995 & Oct & NR & $\mathrm{F}$ & 307 & 500 & 493 & 622 & 0.7926 & 0.562 & 2 \\
\hline Missouri Riv@ Hermann, MO & NMC & 1995 & Oct & NR & $\mathrm{F}$ & 296 & 400 & 416 & 176 & 2.3636 & 1.21 & 2 \\
\hline Missouri Riv@ Hermann, MO & $\mathrm{NMC}$ & 1995 & Oct & NR & M & 435 & 1450 & 395 & 191 & 2.0681 & ND & 2 \\
\hline Missouri Riv@ Hermann, MO & $\mathrm{NMC}$ & 1995 & Oct & NR & M & 353 & 775 & 662 & 2132 & 0.3105 & ND & 2 \\
\hline Missouri Riv@ Hermann, MO & $\mathrm{NMC}$ & 1995 & Oct & NR & M & 320 & 575 & 612 & 463 & 1.3218 & ND & 2 \\
\hline Missouri Riv@ Hermann, MO & $\mathrm{NMC}$ & 1995 & Oct & NR & M & 287 & 400 & 923 & 1295 & 0.7127 & ND & 2 \\
\hline Mohawk Riv@ Frankfort, NY & $\mathrm{NE}$ & 1994 & Sept & NR & $\mathrm{F}$ & 303 & 430 & 561 & 371 & 1.5121 & ND & 1 \\
\hline
\end{tabular}


Table 5. (Continued) All individual LARGEMOUTH BASS values for biomarker and ancillary data from collections made at sites in U.S. waters from March 1994 through August 1997.

[Region: NE, northeast; NW, northwest; SE, southeast; SW, southwest; NMC, northern mid-continent; SMC, southern mid-continent.

Season: NR, non-reproductive. R, reproductive. Sex: F, female, M, male. E2, 17 ß Estradiol; 11KT, 11 ketotestosterone; VTG, vitellogenin; mm, millimeter; g, grams; pg, picograms; mL, milliliter; mg, milligram; ND, not detected; NA, not available]

\begin{tabular}{|c|c|c|c|c|c|c|c|c|c|c|c|c|}
\hline $\begin{array}{l}\text { Site name } \\
\text { (fig. 1) }\end{array}$ & Region & Year & Month & Season & Sex & $\begin{array}{c}\text { Length } \\
(\mathrm{mm})\end{array}$ & $\begin{array}{c}\text { Weight } \\
\text { (g) }\end{array}$ & $\begin{array}{c}E_{2} \\
(\mathrm{pg} / \mathrm{mL})\end{array}$ & $\begin{array}{c}11 \mathrm{KT} \\
(\mathrm{pg} / \mathrm{mL})\end{array}$ & $\begin{array}{l}\text { Ratio of } \\
\text { E}_{2} \text { to KT }\end{array}$ & $\underset{(\mathrm{mg} / \mathrm{mL})}{\text { VTG }}$ & $\begin{array}{c}\text { Develop- } \\
\text { mental } \\
\text { stage }\end{array}$ \\
\hline MohawkRiv@ Frankfort, NY & $\mathrm{NE}$ & 1994 & Sept & NR & $\mathrm{F}$ & 305 & 479 & 172 & 54 & 3.1852 & 0.075 & 1 \\
\hline Mohawk Riv@ Frankfort, NY & $\mathrm{NE}$ & 1994 & Sept & NR & $\mathrm{F}$ & 312 & 534 & 531 & 30 & 17.7 & 0.4 & 1 \\
\hline Mohawk Riv@ Frankfort, NY & $\mathrm{NE}$ & 1994 & Sept & NR & M & 285 & 345 & 44 & 61 & 0.7213 & 0.001 & 3 \\
\hline Mohawk Riv@ Frankfort, NY & $\mathrm{NE}$ & 1994 & Sept & NR & M & 288 & 388 & 256 & 39 & 6.5641 & 0.0012 & 3 \\
\hline Mohawk Riv@ Frankfort, NY & $\mathrm{NE}$ & 1994 & Sept & NR & M & 305 & 420 & 190 & 59 & 3.2203 & 0.0016 & 3 \\
\hline Mohawk Riv@ Frankfort, NY & $\mathrm{NE}$ & 1994 & Sept & NR & M & 277 & 325 & 180 & 48 & 3.75 & 0.215 & 3 \\
\hline OhioRiv@Marietta, OH & $\mathrm{NE}$ & 1995 & Oct & NR & M & 379 & 789 & 409 & 158 & 2.5886 & ND & 2 \\
\hline Ohio Riv@ Metropolis, IL & SMC & 1995 & Oct & NR & $\mathrm{F}$ & 377 & 721 & 2434 & 788 & 3.0888 & ND & 2 \\
\hline Ohio Riv@ Metropolis, IL & SMC & 1995 & Oct & NR & $\mathrm{F}$ & 374 & 615 & 245 & 816 & 0.3002 & ND & 2 \\
\hline Ohio Riv @ Metropolis, IL & SMC & 1995 & Oct & NR & $\mathrm{F}$ & 373 & 613 & 1384 & 731 & 1.8933 & ND & 2 \\
\hline Ohio Riv@ Metropolis, IL & SMC & 1995 & Oct & NR & $\mathrm{F}$ & 345 & 520 & 391 & 856 & 0.4568 & ND & 2 \\
\hline Ohio Riv@ Metropolis, IL & SMC & 1995 & Oct & NR & $\mathrm{F}$ & 344 & 499 & 1313 & 957 & 1.3720 & ND & 2 \\
\hline Ohio Riv @ Metropolis, IL & SMC & 1995 & Oct & NR & $\mathrm{F}$ & 320 & 368 & 469 & 754 & 0.6220 & ND & 2 \\
\hline Ohio Riv@ Metropolis, IL & SMC & 1995 & Oct & NR & $\mathrm{F}$ & 316 & 366 & 355 & 702 & 0.5057 & ND & 2 \\
\hline Ohio Riv@ Metropolis, IL & SMC & 1995 & Oct & NR & $\mathrm{F}$ & 310 & 346 & 1310 & 1312 & 0.9985 & ND & 2 \\
\hline Ohio Riv@ Metropolis, IL & SMC & 1995 & Oct & NR & M & 340 & 470 & 297 & 1077 & 0.2758 & ND & 1 \\
\hline Ohio Riv@ Metropolis, IL & SMC & 1995 & Oct & NR & M & 340 & 424 & 297 & 1338 & 0.2220 & ND & 1 \\
\hline Ohio Riv@ Metropolis, IL & SMC & 1995 & Oct & NR & M & 321 & 409 & 509 & 1397 & 0.3644 & ND & 2 \\
\hline Ohio Riv@ Metropolis, IL & SMC & 1995 & Oct & NR & M & 325 & 405 & 396 & 653 & 0.6064 & ND & 2 \\
\hline Ohio Riv@ Metropolis, IL & SMC & 1995 & Oct & NR & M & 315 & 378 & 596 & 1039 & 0.5736 & ND & 2 \\
\hline Ohio Riv @ Metropolis, IL & SMC & 1995 & Oct & NR & M & 325 & 377 & 226 & 1185 & 0.1907 & ND & 2 \\
\hline Ohio Riv@ Metropolis, IL & SMC & 1995 & Oct & NR & M & 305 & 321 & 385 & 1236 & 0.3115 & ND & 1 \\
\hline Ohio Riv@ Metropolis, IL & SMC & 1995 & Oct & NR & M & 249 & 176 & 199 & 418 & 0.4761 & ND & 1 \\
\hline Ohio Riv@ Metropolis, IL & SMC & 1995 & Oct & NR & M & 350 & 555 & 365 & 1298 & 0.2812 & 0.005 & 2 \\
\hline Potomic Riv@ Anacostia Riv & $\mathrm{NE}$ & 1994 & Nov & NR & $\mathrm{F}$ & 293 & 354 & 235 & 232 & 1.0129 & 0.0016 & 1 \\
\hline Potomic Riv@ Anacostia Riv & $\mathrm{NE}$ & 1994 & Nov & NR & $\mathrm{F}$ & 298 & 365 & 172 & 97 & 1.7732 & 0.056 & 1 \\
\hline Potomic Riv@Anacostia Riv & $\mathrm{NE}$ & 1994 & Nov & NR & $\mathrm{F}$ & 320 & 503 & 546 & 731 & 0.7469 & 0.117 & 1 \\
\hline Potomic Riv@ Anacostia Riv & $\mathrm{NE}$ & 1994 & Nov & NR & $\mathrm{F}$ & 400 & 1164 & 136 & 240 & 0.5667 & 0.18 & 1 \\
\hline
\end{tabular}


Table 5. (Continued) All individual LARGEMOUTH BASS values for biomarker and ancillary data from collections made at sites in U.S. waters from March 1994 through August 1997.

[Region: NE, northeast; NW, northwest; SE, southeast; SW, southwest; NMC, northern mid-continent; SMC, southern mid-continent.

Season: NR, non-reproductive. R, reproductive. Sex: F, female, M, male. E2, 17 B Estradiol; 11KT, 11 ketotestosterone; VTG, vitellogenin; mm, millimeter; g, grams; pg, picograms; mL, milliliter; mg, milligram; ND, not detected; NA, not available]

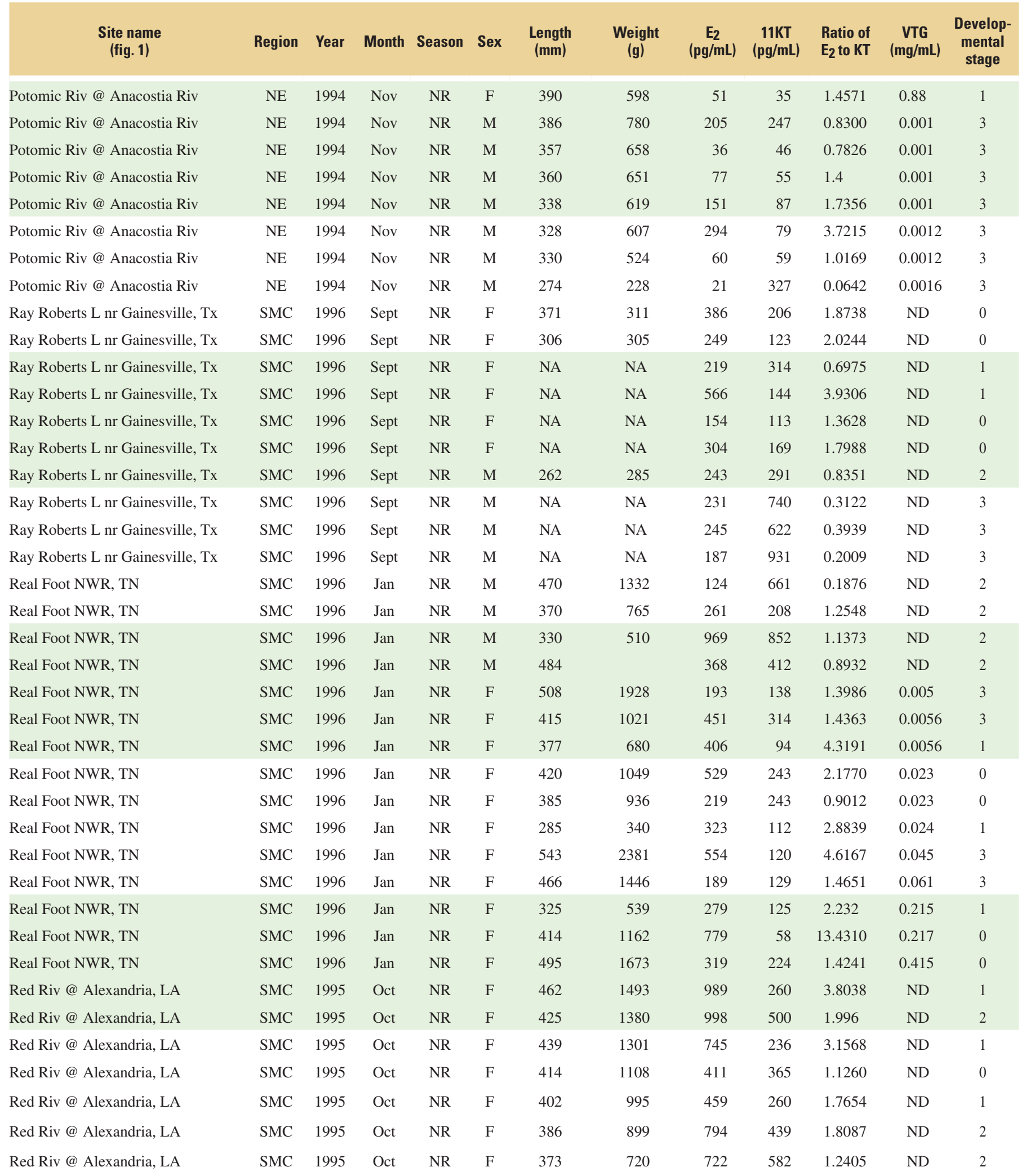


Table 5. (Continued) All individual LARGEMOUTH BASS values for biomarker and ancillary data from collections made at sites in U.S. waters from March 1994 through August 1997.

[Region: NE, northeast; NW, northwest; SE, southeast; SW, southwest; NMC, northern mid-continent; SMC, southern mid-continent.

Season: NR, non-reproductive. R, reproductive. Sex: F, female, M, male. E2, 17 B Estradiol; 11KT, 11 ketotestosterone; VTG, vitellogenin; mm, millimeter; g, grams; pg, picograms; mL, milliliter; mg, milligram; ND, not detected; NA, not available]

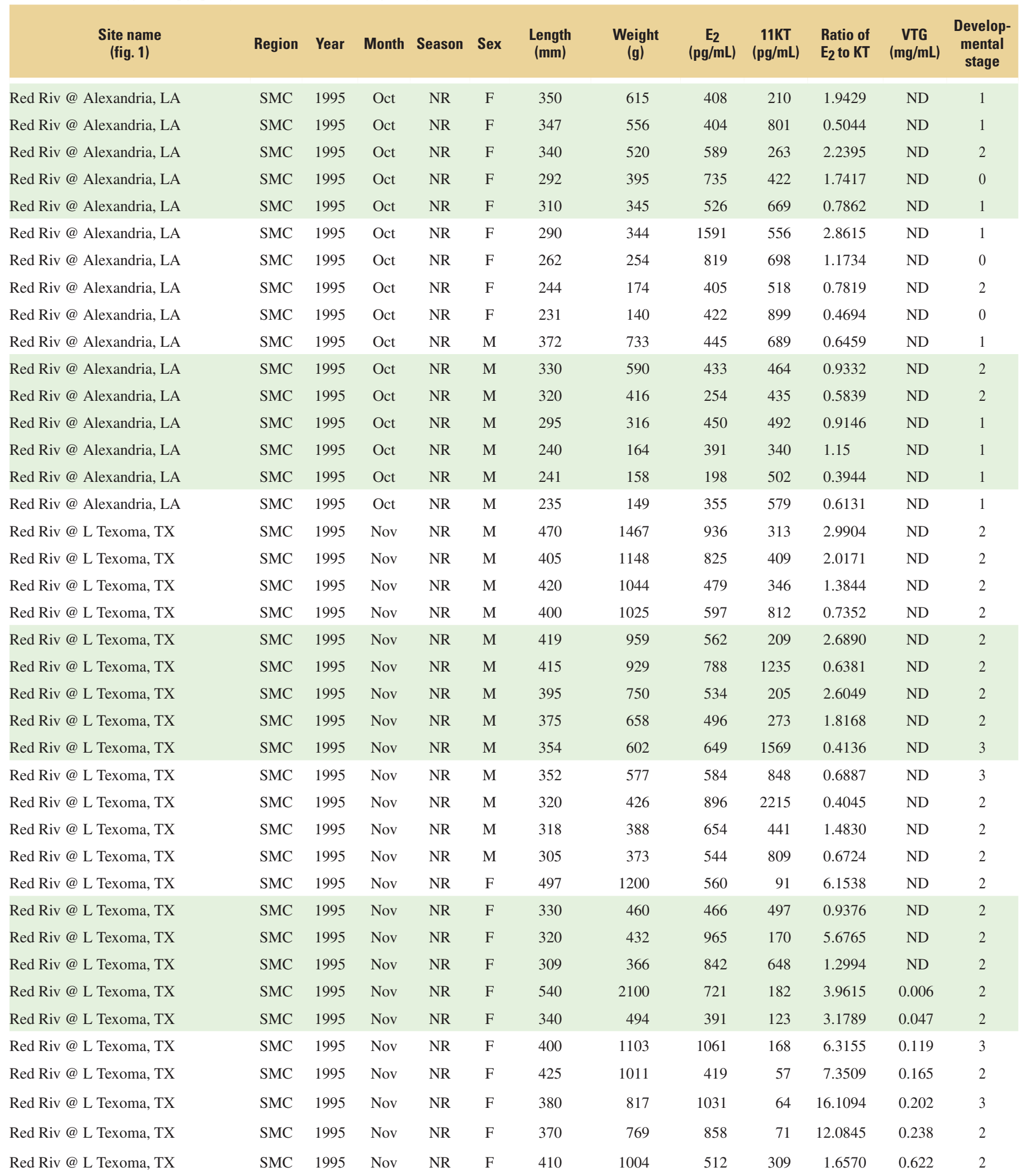


Table 5. (Continued) All individual LARGEMOUTH BASS values for biomarker and ancillary data from collections made at sites in U.S. waters from March 1994 through August 1997.

[Region: NE, northeast; NW, northwest; SE, southeast; SW, southwest; NMC, northern mid-continent; SMC, southern mid-continent.

Season: NR, non-reproductive. R, reproductive. Sex: F, female, M, male. E2, 17 B Estradiol; 11KT, 11 ketotestosterone; VTG, vitellogenin; mm, millimeter; g, grams; pg, picograms; mL, milliliter; mg, milligram; ND, not detected; NA, not available]

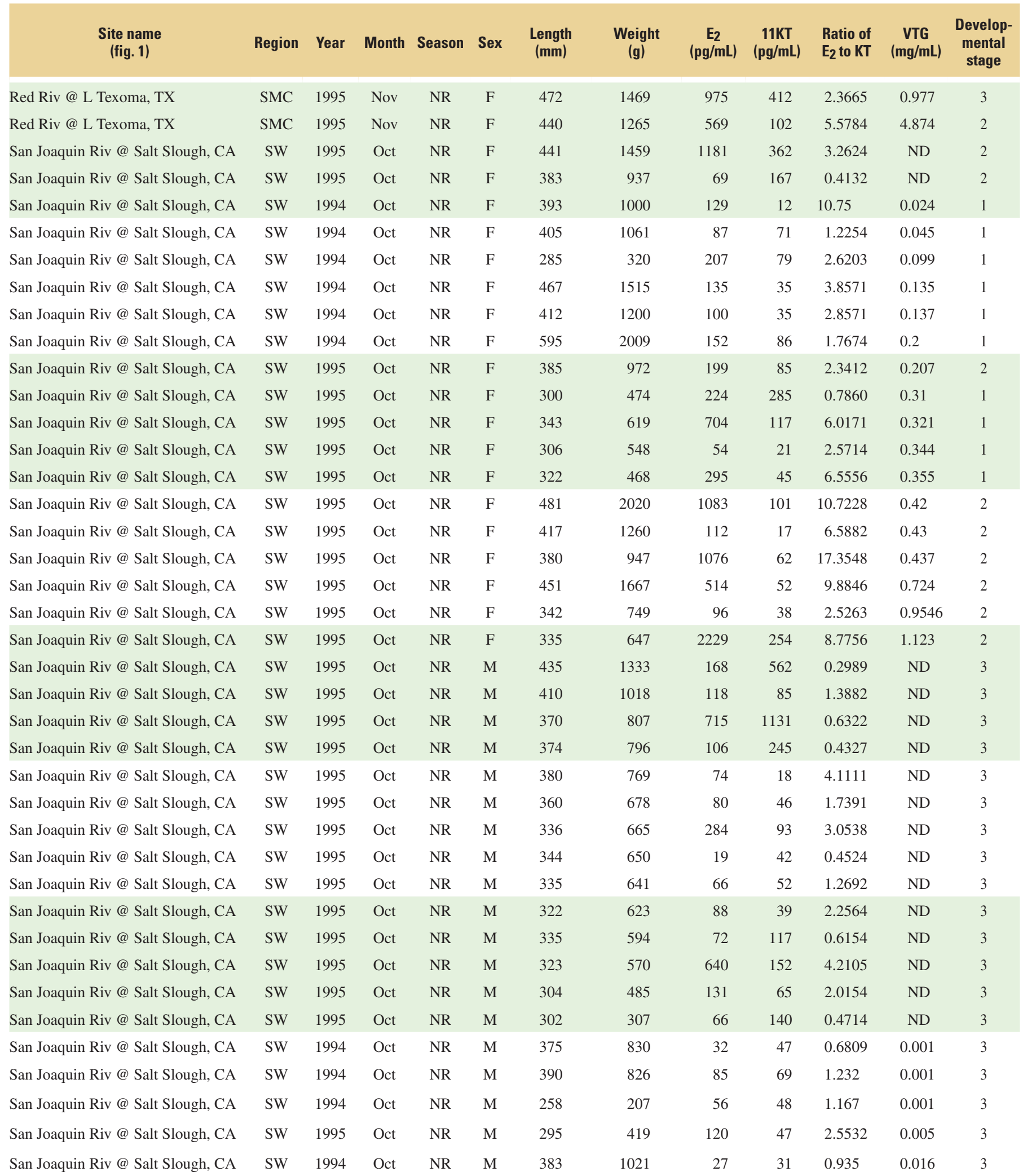


Table 5. (Continued) All individual LARGEMOUTH BASS values for biomarker and ancillary data from collections made at sites in U.S. waters from March 1994 through August 1997.

[Region: NE, northeast; NW, northwest; SE, southeast; SW, southwest; NMC, northern mid-continent; SMC, southern mid-continent.

Season: NR, non-reproductive. R, reproductive. Sex: F, female, M, male. E2, 17 ß Estradiol; 11KT, 11 ketotestosterone; VTG, vitellogenin; mm, millimeter; g, grams; pg, picograms; mL, milliliter; mg, milligram; ND, not detected; NA, not available]

\begin{tabular}{|c|c|c|c|c|c|c|c|c|c|c|c|c|}
\hline $\begin{array}{l}\text { Site name } \\
\text { (fig. 1) }\end{array}$ & Region & Year & Month & Season & Sex & $\begin{array}{l}\text { Length } \\
(\mathrm{mm})\end{array}$ & $\begin{array}{c}\text { Weight } \\
\text { (g) }\end{array}$ & $\begin{array}{c}E_{2} \\
(\mathrm{pg} / \mathrm{mL})\end{array}$ & $\begin{array}{c}11 \mathrm{KT} \\
(\mathrm{pg} / \mathrm{mL})\end{array}$ & $\begin{array}{l}\text { Ratio of } \\
E_{2} \text { to KT }\end{array}$ & $\underset{(\mathrm{mg} / \mathrm{mL})}{\text { VTG }}$ & $\begin{array}{c}\text { Develop- } \\
\text { mental } \\
\text { stage }\end{array}$ \\
\hline San Joaquin Riv@ Salt Slough, CA & SW & 1995 & Oct & NR & M & 328 & 596 & 151 & 91 & 1.6593 & 0.039 & 3 \\
\hline San Joaquin Riv@ Vernalis, CA & SW & 1994 & Oct & NR & $\mathrm{F}$ & 442 & 1401 & 377 & 165 & 2.2848 & ND & 1 \\
\hline San Joaquin Riv@ Vernalis, CA & SW & 1994 & Oct & NR & $\mathrm{F}$ & 357 & 627 & 187 & 148 & 1.2635 & ND & 1 \\
\hline San Joaquin Riv@ Vernalis, CA & SW & 1995 & Oct & NR & $\mathrm{F}$ & 324 & 522 & 1345 & 241 & 5.5809 & ND & 1 \\
\hline San Joaquin Riv@ Vernalis, CA & SW & 1994 & Oct & NR & $\mathrm{F}$ & 320 & 457 & 281 & 132 & 2.1288 & 0.002 & 1 \\
\hline San Joaquin Riv@ Vernalis, CA & SW & 1994 & Oct & NR & $\mathrm{F}$ & 376 & 800 & 284 & 111 & 2.5586 & 0.004 & 1 \\
\hline San Joaquin Riv@ Vernalis, CA & SW & 1994 & Oct & NR & $\mathrm{F}$ & 320 & 419 & 116 & 127 & 0.9134 & 0.039 & 1 \\
\hline San Joaquin Riv@ Vernalis, CA & SW & 1994 & Oct & NR & $\mathrm{F}$ & 350 & 678 & 200 & 114 & 1.7544 & 0.046 & 1 \\
\hline San Joaquin Riv@ Vernalis, CA & SW & 1994 & Oct & NR & $\mathrm{F}$ & 270 & 809 & 170 & 137 & 1.2409 & 0.049 & 1 \\
\hline San Joaquin Riv@ Vernalis, CA & SW & 1994 & Oct & NR & $\mathrm{F}$ & 482 & 2010 & 93 & 117 & 0.7949 & 0.05 & 1 \\
\hline San Joaquin Riv@ Vernalis, CA & SW & 1994 & Oct & NR & $\mathrm{F}$ & 349 & 643 & 175 & 204 & 0.8578 & 0.052 & 1 \\
\hline San Joaquin Riv@ Vernalis, CA & SW & 1995 & Oct & NR & $\mathrm{F}$ & 311 & 463 & 95 & 159 & 0.5975 & 0.209 & 2 \\
\hline San Joaquin Riv@ Vernalis,CA & SW & 1995 & Oct & NR & $\mathrm{F}$ & 422 & 1043 & 405 & 527 & 0.7685 & 0.21 & 2 \\
\hline San Joaquin Riv@ Vernalis,CA & SW & 1995 & Oct & NR & $\mathrm{F}$ & 361 & 605 & 1243 & 133 & 9.3459 & 0.21 & 2 \\
\hline San Joaquin Riv@ Vernalis, CA & SW & 1995 & Oct & NR & $\mathrm{F}$ & 465 & 1583 & 344 & 310 & 1.1097 & 0.217 & 1 \\
\hline San Joaquin Riv@ Vernalis, CA & SW & 1995 & Oct & NR & $\mathrm{F}$ & 426 & 1125 & 310 & 85 & 3.6471 & 0.597 & 1 \\
\hline San Joaquin Riv @ Vernalis, CA & SW & 1995 & Oct & NR & $\mathrm{F}$ & 373 & 851 & 259 & 353 & 0.7337 & 0.853 & 1 \\
\hline San Joaquin Riv@ Vernalis,CA & SW & 1995 & Oct & NR & $\mathrm{F}$ & 356 & 773 & 208 & 429 & 0.4848 & 0.956 & 2 \\
\hline San Joaquin Riv@ Vernalis, CA & SW & 1995 & Oct & NR & $\mathrm{F}$ & 448 & 1292 & 326 & 515 & 0.6330 & 1.259 & 2 \\
\hline San Joaquin Riv@ Vernalis, CA & SW & 1995 & Oct & NR & $\mathrm{F}$ & 346 & 674 & 1245 & 267 & 4.6629 & 1.288 & 2 \\
\hline San Joaquin Riv@ Vernalis,CA & SW & 1995 & Oct & NR & $\mathrm{F}$ & 428 & 1395 & 114 & 110 & 1.0364 & 3.541 & 2 \\
\hline San Joaquin Riv@ Vernalis, CA & SW & 1995 & Oct & NR & M & 378 & 870 & 141 & 144 & 0.9792 & ND & 3 \\
\hline San Joaquin Riv@ Vernalis, CA & SW & 1995 & Oct & NR & M & 356 & 761 & 243 & 403 & 0.6030 & ND & 3 \\
\hline San Joaquin Riv@ Vernalis, CA & SW & 1995 & Oct & NR & M & 354 & 732 & 131 & 181 & 0.7238 & ND & 3 \\
\hline San Joaquin Riv@ Vernalis, CA & SW & 1995 & Oct & NR & M & 354 & 678 & 761 & 190 & 4.0053 & ND & 3 \\
\hline San Joaquin Riv@ Vernalis, CA & SW & 1995 & Oct & NR & M & 375 & 654 & 77 & 323 & 0.2384 & ND & 3 \\
\hline San Joaquin Riv@ Vernalis, CA & SW & 1995 & Oct & NR & M & 357 & 627 & 439 & 618 & 0.7104 & ND & 3 \\
\hline San Joaquin Riv@ Vernalis, CA & SW & 1995 & Oct & NR & M & 328 & 553 & 77 & 128 & 0.6016 & ND & 3 \\
\hline
\end{tabular}


Table 5. (Continued) All individual LARGEMOUTH BASS values for biomarker and ancillary data from collections made at sites in U.S. waters from March 1994 through August 1997.

[Region: NE, northeast; NW, northwest; SE, southeast; SW, southwest; NMC, northern mid-continent; SMC, southern mid-continent.

Season: NR, non-reproductive. R, reproductive. Sex: F, female, M, male. E2, 17 B Estradiol; 11KT, 11 ketotestosterone; VTG, vitellogenin; mm, millimeter; g, grams; pg, picograms; mL, milliliter; mg, milligram; ND, not detected; NA, not available]

\begin{tabular}{|c|c|c|c|c|c|c|c|c|c|c|c|c|}
\hline $\begin{array}{c}\text { Site name } \\
\text { (fig. 1) }\end{array}$ & Region & Year & Month & Season & Sex & $\begin{array}{l}\text { Length } \\
(\mathrm{mm})\end{array}$ & $\begin{array}{c}\text { Weight } \\
\text { (g) }\end{array}$ & $\underset{(p g / m L)}{E_{2}}$ & $\begin{array}{c}11 \mathrm{KT} \\
(\mathrm{pg} / \mathrm{mL})\end{array}$ & $\begin{array}{l}\text { Ratio of } \\
E_{2} \text { to KT }\end{array}$ & $\underset{(\mathrm{mg} / \mathrm{mL})}{\text { VTG }}$ & $\begin{array}{c}\text { Develop- } \\
\text { mental } \\
\text { stage }\end{array}$ \\
\hline San Joaquin Riv @ Vernalis, CA & SW & 1995 & Oct & NR & M & 311 & 467 & 339 & 197 & 1.7208 & ND & 3 \\
\hline San Joaquin Riv @ Vernalis, CA & SW & 1995 & Oct & NR & M & 291 & 360 & 209 & 656 & 0.3186 & ND & 3 \\
\hline San Joaquin Riv @ Vernalis, CA & SW & 1994 & Oct & NR & M & 406 & 993 & 154 & 573 & 0.2688 & 0.0016 & 3 \\
\hline San Joaquin Riv @ Vernalis, CA & SW & 1994 & Oct & NR & M & 355 & 692 & 148 & 152 & 0.9737 & 0.0016 & 3 \\
\hline San Joaquin Riv@ Vernalis, CA & SW & 1994 & Oct & NR & M & 356 & 646 & 35 & 181 & 0.1934 & 0.0018 & 3 \\
\hline San Joaquin Riv @ Vernalis, CA & SW & 1994 & Oct & NR & M & 355 & 789 & 257 & 103 & 2.4951 & 0.002 & 3 \\
\hline San Joaquin Riv @ Vernalis, CA & SW & 1994 & Oct & NR & M & 226 & 230 & 141 & 207 & 0.6812 & 0.117 & 3 \\
\hline Snake Riv@ Hagerman, ID & NW & 1997 & Oct & NR & $\mathrm{F}$ & 430 & 1150 & 743 & 179 & 4.1508 & 0.026 & 1 \\
\hline SnakeRiv@ Hagerman, ID & NW & 1997 & Oct & NR & $\mathrm{F}$ & 492 & 2000 & 412 & 167 & 2.4671 & 0.031 & 1 \\
\hline Snake Riv@ Hagerman, ID & NW & 1997 & Oct & NR & $\mathrm{F}$ & 318 & 484 & 1134 & 481 & 2.3576 & 0.031 & 1 \\
\hline SnakeRiv@ Hagerman, ID & NW & 1997 & Oct & NR & $\mathrm{F}$ & 334 & 714 & 591 & 454 & 1.3018 & 0.058 & 1 \\
\hline Snake Riv@ Hagerman, ID & NW & 1997 & Oct & NR & $\mathrm{F}$ & 367 & 914 & 413 & 377 & 1.0955 & 0.059 & 2 \\
\hline Snake Riv@ Hagerman, ID & NW & 1997 & Oct & NR & $\mathrm{F}$ & 394 & 1125 & 797 & 208 & 3.8317 & 0.069 & 1 \\
\hline SnakeRiv@ Hagerman, ID & NW & 1997 & Oct & NR & $\mathrm{F}$ & 330 & 531 & 242 & 191 & 1.2670 & 0.071 & 1 \\
\hline SnakeRiv@ Hagerman, ID & NW & 1997 & Oct & NR & $\mathrm{F}$ & 257 & 253 & 871 & 342 & 2.5468 & 0.408 & 1 \\
\hline SnakeRiv@ Hagerman, ID & NW & 1997 & Oct & NR & M & 314 & 500 & 310 & 7451 & 0.0416 & ND & 2 \\
\hline SnakeRiv@ Hagerman, ID & NW & 1997 & Oct & NR & M & 315 & 444 & 582 & 711 & 0.8186 & ND & 3 \\
\hline Swinging Brg Res nr Monticello, NY & $\mathrm{NE}$ & 1994 & Oct & NR & $\mathrm{F}$ & 448 & NA & 446 & 244 & 1.8279 & 0.006 & 2 \\
\hline Swinging Brg Res nr Monticello, NY & NE & 1994 & Oct & NR & $\mathrm{F}$ & 391 & 956 & 211 & 129 & 1.6357 & 0.09 & 1 \\
\hline Swinging Brg Res nr Monticello, NY & NE & 1994 & Oct & NR & $\mathrm{F}$ & 383 & 930 & 503 & 75 & 6.7067 & 0.136 & 2 \\
\hline Swinging Brg Res nr Monticello, NY & $\mathrm{NE}$ & 1994 & Oct & NR & $\mathrm{F}$ & 359 & NA & 701 & 46 & 15.2391 & 0.196 & 2 \\
\hline Swinging Brg Res nr Monticello, NY & $\mathrm{NE}$ & 1994 & Oct & NR & $\mathrm{F}$ & 442 & NA & 380 & 75 & 5.0667 & 0.2 & 2 \\
\hline Swinging Brg Res nr Monticello, NY & $\mathrm{NE}$ & 1994 & Oct & NR & $\mathrm{F}$ & 495 & NA & 124 & 92 & 1.3478 & 0.24 & 2 \\
\hline Swinging Brg Res nr Monticello, NY & $\mathrm{NE}$ & 1994 & Oct & NR & $\mathrm{F}$ & 370 & 785 & 741 & 148 & 5.007 & 0.4 & 1 \\
\hline Swinging Brg Res nr Monticello, NY & $\mathrm{NE}$ & 1994 & Oct & NR & M & 374 & 850 & 63 & 76 & 0.8289 & ND & 3 \\
\hline Swinging Brg Res nr Monticello, NY & $\mathrm{NE}$ & 1994 & Oct & NR & M & 345 & 581 & 20 & 63 & 0.3175 & ND & 3 \\
\hline Swinging Brg Res nr Monticello, NY & $\mathrm{NE}$ & 1994 & Oct & NR & M & 350 & NA & 14 & 149 & 0.0940 & ND & 3 \\
\hline Swinging Brg Res nr Monticello, NY & $\mathrm{NE}$ & 1994 & Oct & NR & M & 343 & NA & 52 & 212 & 0.2453 & ND & 3 \\
\hline
\end{tabular}


Table 5. (Continued) All individual LARGEMOUTH BASS values for biomarker and ancillary data from collections made at sites in U.S. waters from March 1994 through August 1997.

[Region: NE, northeast; NW, northwest; SE, southeast; SW, southwest; NMC, northern mid-continent; SMC, southern mid-continent.

Season: NR, non-reproductive. R, reproductive. Sex: F, female, M, male. E2, 17 B Estradiol; 11KT, 11 ketotestosterone; VTG, vitellogenin; mm, millimeter; g, grams; pg, picograms; mL, milliliter; mg, milligram; ND, not detected; NA, not available]

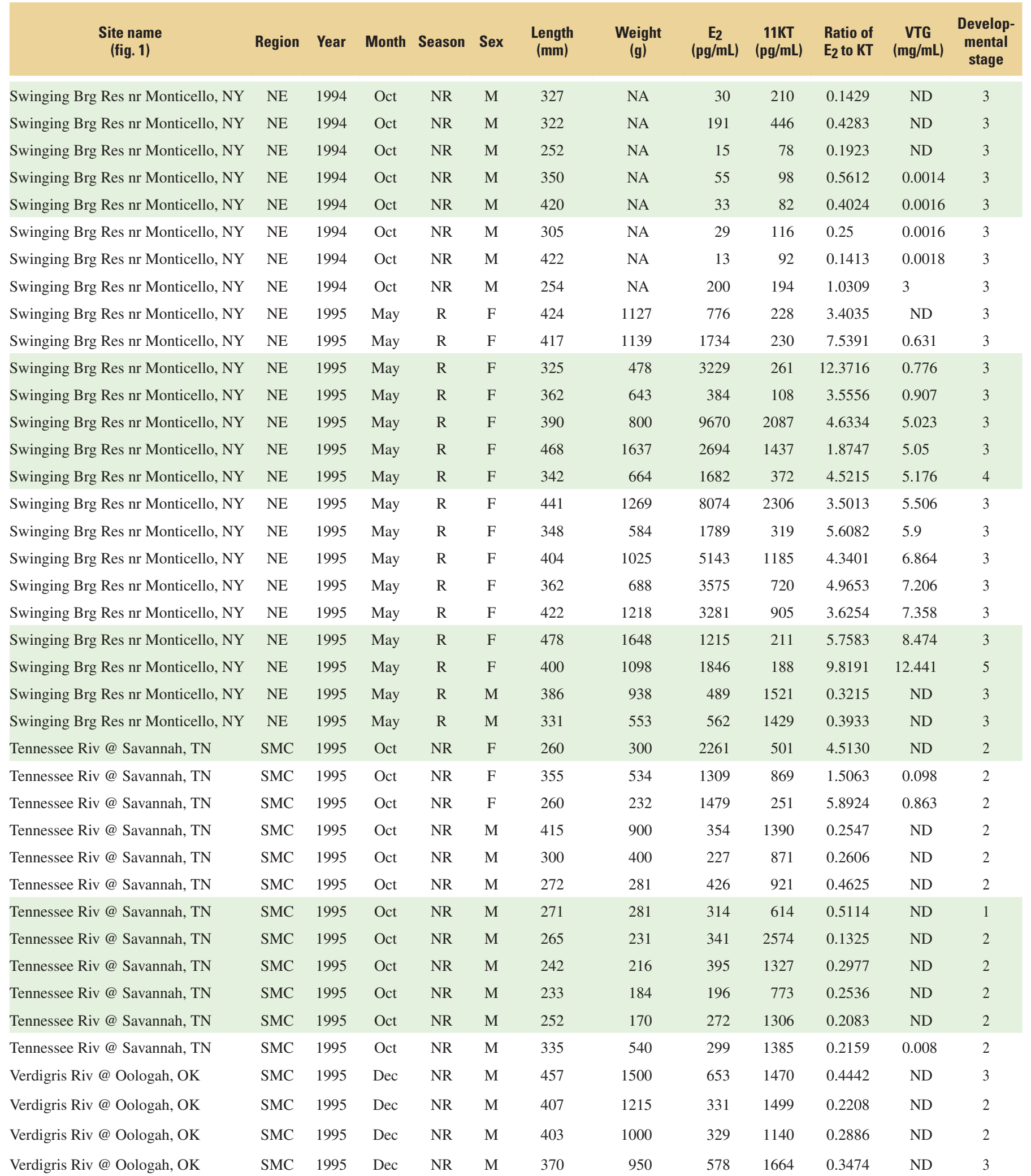


Table 5. (Continued) All individual LARGEMOUTH BASS values for biomarker and ancillary data from collections made at sites in U.S. waters from March 1994 through August 1997.

[Region: NE, northeast; NW, northwest; SE, southeast; SW, southwest; NMC, northern mid-continent; SMC, southern mid-continent.

Season: NR, non-reproductive. R, reproductive. Sex: F, female, M, male. E2, 17 B Estradiol; 11KT, 11 ketotestosterone; VTG, vitellogenin; mm, millimeter; g, grams; pg, picograms; mL, milliliter; mg, milligram; ND, not detected; NA, not available]

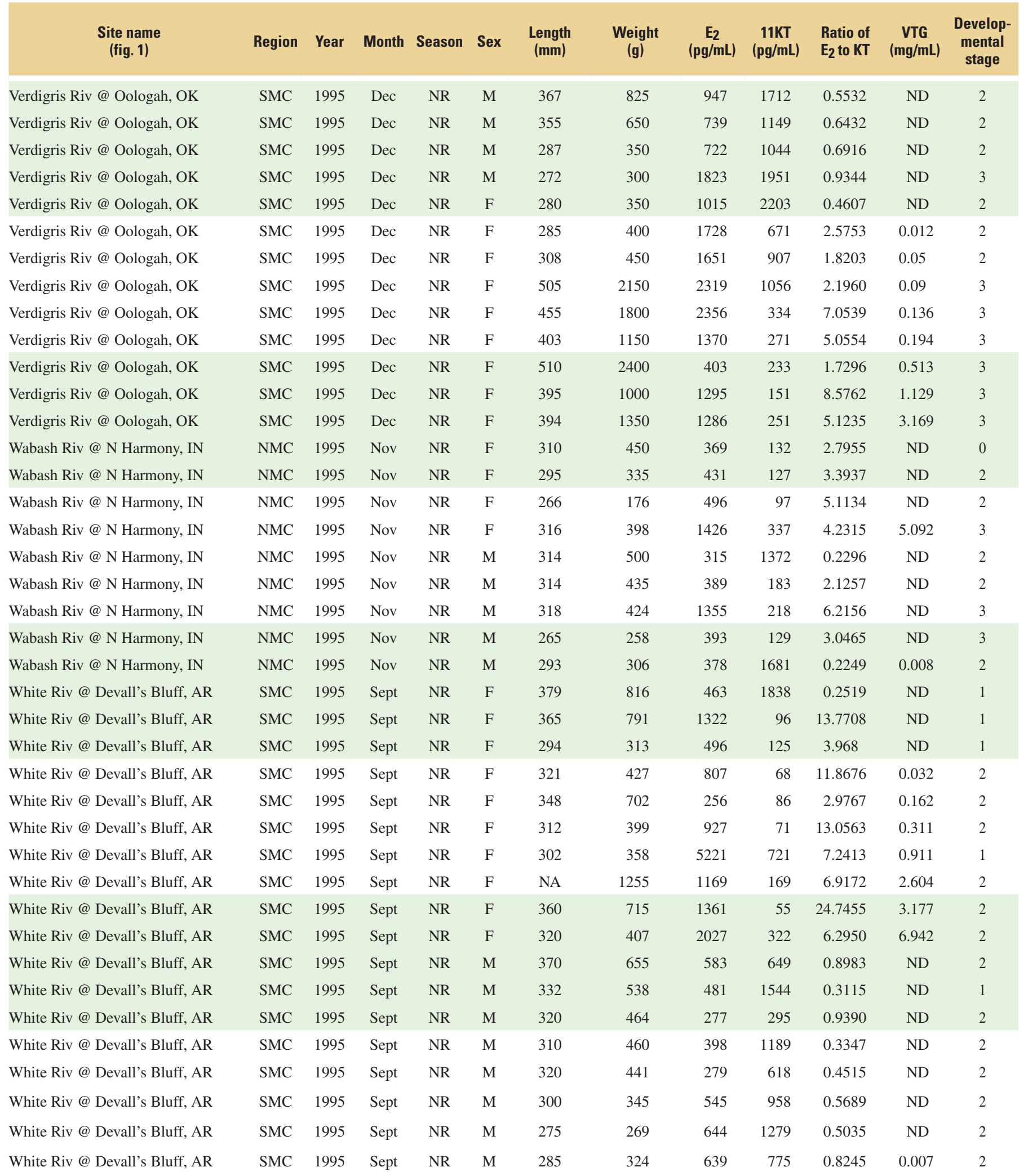


Table 5. (Continued) All individual LARGEMOUTH BASS values for biomarker and ancillary data from collections made at sites in U.S. waters from March 1994 through August 1997.

[Region: NE, northeast; NW, northwest; SE, southeast; SW, southwest; NMC, northern mid-continent; SMC, southern mid-continent.

Season: NR, non-reproductive. R, reproductive. Sex: F, female, M, male. E2, 17 ß Estradiol; 11KT, 11 ketotestosterone; VTG, vitellogenin; mm, millimeter; g, grams; pg, picograms; mL, milliliter; mg, milligram; ND, not detected; NA, not available]

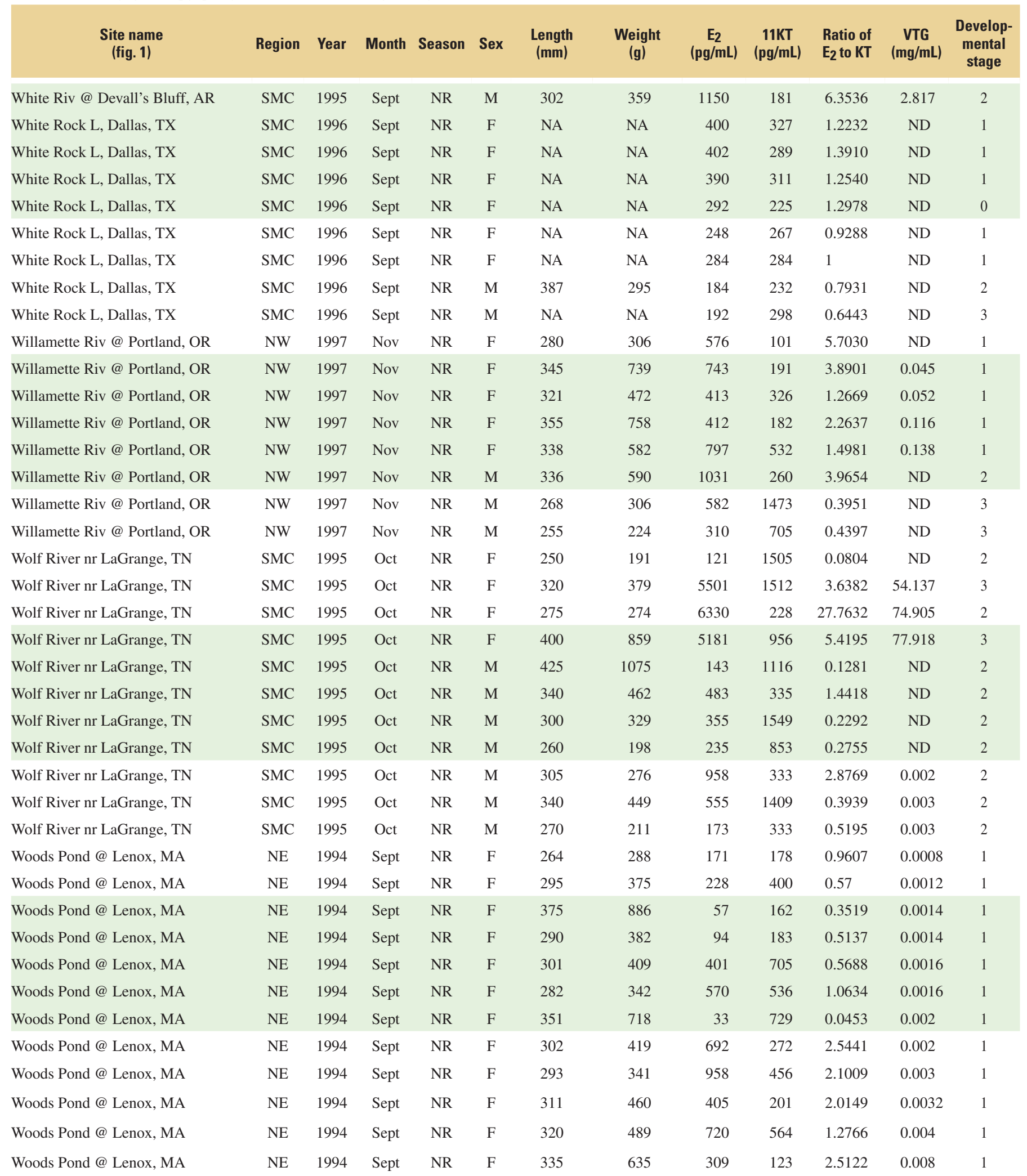


Table 5. (Continued) All individual LARGEMOUTH BASS values for biomarker and ancillary data from collections made at sites in U.S. waters from March 1994 through August 1997.

[Region: NE, northeast; NW, northwest; SE, southeast; SW, southwest; NMC, northern mid-continent; SMC, southern mid-continent.

Season: NR, non-reproductive. R, reproductive. Sex: F, female, M, male. E2, 17 B Estradiol; 11KT, 11 ketotestosterone; VTG, vitellogenin; mm, millimeter; g, grams; pg, picograms; $\mathrm{mL}$, milliliter; mg, milligram; ND, not detected; NA, not available]

\begin{tabular}{|c|c|c|c|c|c|c|c|c|c|c|c|c|}
\hline $\begin{array}{c}\text { Site name } \\
\text { (fig. 1) }\end{array}$ & Region & Year & Month & Season & Sex & $\begin{array}{c}\text { Length } \\
\text { (mm) }\end{array}$ & $\begin{array}{c}\text { Weight } \\
\text { (g) }\end{array}$ & $\begin{array}{c}E_{2} \\
(p g / m L)\end{array}$ & $\begin{array}{c}11 \mathrm{KT} \\
(\mathrm{pg} / \mathrm{mL})\end{array}$ & $\begin{array}{l}\text { Ratio of } \\
E_{2} \text { to KT }\end{array}$ & $\begin{array}{c}\text { VTG } \\
\text { (mg/mL) }\end{array}$ & $\begin{array}{c}\text { Develop- } \\
\text { mental } \\
\text { stage }\end{array}$ \\
\hline Woods Pond@Lenox, MA & $\mathrm{NE}$ & 1994 & Sept & NR & M & 251 & 248 & 795 & 190 & 4.184 & 0.0012 & 3 \\
\hline Woods Pond@ Lenox, MA & $\mathrm{NE}$ & 1994 & Sept & NR & M & 335 & 542 & 107 & 217 & 0.4931 & 0.0014 & 3 \\
\hline Woods Pond @ Lenox, MA & $\mathrm{NE}$ & 1994 & Sept & NR & M & 318 & 519 & 275 & 509 & 0.5403 & 0.002 & 0 \\
\hline Woods Pond @ Lenox, MA & $\mathrm{NE}$ & 1994 & Sept & NR & M & 272 & 290 & 601 & 807 & 0.745 & 0.0036 & 2 \\
\hline Woods Pond @ Lenox, MA & $\mathrm{NE}$ & 1995 & May & $\mathrm{R}$ & F & 286 & 354 & 732 & 402 & 1.8209 & ND & 3 \\
\hline Woods Pond @ Lenox, MA & $\mathrm{NE}$ & 1995 & May & $\mathrm{R}$ & $\mathrm{F}$ & 293 & 350 & 965 & 554 & 1.7419 & 0.718 & 2 \\
\hline Woods Pond@Lenox, MA & $\mathrm{NE}$ & 1995 & May & $\mathrm{R}$ & M & 307 & 510 & 449 & 583 & 0.7702 & ND & 3 \\
\hline Woods Pond @ Lenox, MA & $\mathrm{NE}$ & 1995 & May & $\mathrm{R}$ & M & 315 & 453 & 560 & 684 & 0.8187 & ND & 3 \\
\hline Woods Pond@Lenox, MA & $\mathrm{NE}$ & 1995 & May & $\mathrm{R}$ & M & 293 & 437 & 1209 & 1545 & 0.7825 & ND & 3 \\
\hline Woods Pond @ Lenox, MA & $\mathrm{NE}$ & 1995 & May & $\mathrm{R}$ & M & 290 & 408 & 379 & 1706 & 0.2222 & ND & 3 \\
\hline Woods Pond@ Lenox, MA & $\mathrm{NE}$ & 1995 & May & $\mathrm{R}$ & M & 308 & 405 & 280 & 1117 & 0.2507 & ND & 3 \\
\hline Woods Pond @ Lenox, MA & $\mathrm{NE}$ & 1995 & May & $\mathrm{R}$ & M & 305 & 389 & 1621 & 845 & 1.9183 & ND & 3 \\
\hline Woods Pond @ Lenox, MA & $\mathrm{NE}$ & 1995 & May & $\mathrm{R}$ & M & 280 & 345 & 1246 & 410 & 3.0390 & ND & 3 \\
\hline Woods Pond @ Lenox, MA & $\mathrm{NE}$ & 1995 & May & $\mathrm{R}$ & M & 276 & 335 & 531 & 1679 & 0.3163 & ND & 3 \\
\hline Woods Pond@Lenox, MA & $\mathrm{NE}$ & 1995 & May & $\mathrm{R}$ & M & 282 & 331 & 1372 & 1114 & 1.2316 & ND & 3 \\
\hline Yakima Riv@ Granger, WA & NW & 1997 & Oct & NR & M & 418 & 1138 & 106 & 199 & 0.5327 & ND & 3 \\
\hline Yakima Riv@ Granger, WA & NW & 1997 & Oct & NR & M & 356 & 691 & 179 & 435 & 0.4115 & ND & 2 \\
\hline
\end{tabular}




\section{References Cited}

Baumann, P.C., Mac, M.J., Smith, S.B., and Harshbarger, J.C., 1991, Tumor frequencies in walleye and brown bullhead and sediment contaminants in tributaries of the Laurentian Great Lakes: Canadian Journal of Fisheries and Aquatic Sciences, v. 48, p. 1804-1810.

Bevans, H.E., Goodbred, S.L., Miesner, J.F., Watkins, S.A., Gross, T.S., Denslow, N.D., and others., 1996, Synthetic organic compounds and carp endocrinology and histology in Las Vegas wash and Las Vegas Callville Bays of Lake Mead, Nevada, 1992 and 1995: U.S. Geological Survey, Water-Resources Investigations Report 96-4266, 12 p.

Borg, B., 1994, Androgens in teleost fishes: Comparative Biochemisty and Physiology, v. 109C, p. 219-245.

Bromage, N., and Cumaranatunga, R., 1998, Egg production in the rainbow trout, in Muir, J.F., and Roberts, R.J., eds., Recent advances in aquaculture: v. 3, p. 63-138.

Cavaco, J.E.B, Vilrokx, C.V.L., Trudeau, R.W., and Schulz, H.J., 1998, Sex steroids and the initiation of puberty in male African catfish (Clarias gariepinus): American Journal of Physiology, v. 275, p. R1793-R1802.

Cavaco, J.E.B., Schulz, R.W., and Goos, H.J.T., 1999, Steroid hormones associated with changes in steroidogenesis and Leydig cell ultrastructure in juvenile African catfish, (Clarias gariepinus): Cell and Tissue Research, v. 297, p. 291-299.

Denslow, N.D., Chow, M., Chow, M.C., Bonomelli, S., Folmar, L.C., Heppell, S.A., and Sullivan, C.V., 1997, Development of biomarkers for environmental contaminants affecting fish, in Rolland, R.M., Gilbertson, M., and Peterson, R.E., eds., Chemically induced alterations in functional development and reproduction of fishes: Pensacola, Fla., SETAC Press, p. 73-86.

Denslow, N.D., Chow M., Chow, M.C., Folmar, L.C., Monomelli, S., Heppell, S.A., and Sulliva, C.V., 1996a, Development of antibodies to teleost vitellogenins: Potential biomarkers for environmental estrogens, in Bengston, D.A., and Henshel, D.S., eds., Environmental Toxicology and Risk Assessment: Philadelphia, American Society for Testing and Materials, v. ASTM STP 13065, p. 23-36.

Denslow, N.D., Chow, M.C., Kroll, K.J., and Green, L., 1996b, Vitellogenin as a biomarker of exposure for estrogen or estrogen mimics: Ecotoxicology, v. 8, p. 385-398.

Denslow, N.D., Chow, M.C., Kroll, K.J., and. Green. L., 1999, Vitellogenin as a biomarker for estrogen or estrogen mimics: Ecotoxicology, v. 8, p. 385-398.

Down, N.E., Peter, R.E., and Leatherland, J.F., 1990, Seasonal changes in serum gonadotropin, testosterone, 11-ketotestosterone, and estradiol-17ß levels and their relation to tumor burdening on adult tumor bearing carp x goldfish hybrids in the Great Lakes: General and Comparative Endocrinology, v. 77, p. 192.

Gilliom, R.J., Alley, W.M., Gurtz, M.E., 1995, Design of the National Water Quality Assessment program: Occurrence and distribution of water-quality conditions: U.S. Geological Survey Circular 1112, 33 p.

Goodbred, S.L., Gilliom, R.J., Gross, T.S., Denslow, N.D., Bryant, W.B., and Schoeb T.R., 1997, Reconnaissance of $17 \beta$-estradiol, 11-ketotestosterone, vitellogenin, and gonad histopathology in common carp of United States streams: Potential for contaminant induced endocrine disruption: U.S. Geological Survey Open-File Report 96-627, 47 p.

Gross, T., Arnold, B.S., Sepulveda, M.S., McDonald, K., 2003, Endocrine disrupting chemicals and endocrine active agents, in Hoffman, Rattner, B., Burton, G.A., Jr., and Cairns, J., Jr., eds., Handbook of Ecotoxicology ( 2 d ed.): Boca Raton, Fla., CRC Press, chap. 39, p. 1033-1098.

Gross, T.S., Sepulveda, M.S., Wieser, C.M., Wiebe, J.J., Schoeb, T.R., Denslow, N.D., 2002, Characterization of annual reproductive cycles for pond-reared Florida largemouth bass (Micropterus salmonides floridanus): American Fisheries Society Symposium, v. 31, p. 205-212.

Harrison, E.Z., McBride, M.B., and Bouldin, D.R., 1999, Land application of sewage sludges: An appraisal of the U.S. regulations: Internal Journal of Environment and Pollution, v. 11, p. 1.

Heppell, J., Tarrab, E., Lecomte, J., Berthiaume, L., and Arella, M., 1995, Strain variability and localization of important epitopes on the major structural protein (VP2) of infectious pancreatic necrosis virus: Virology, v. 214, p. 40-49.

Hoffman, D.J., Rattner, B.A., Burton, G.A., Jr., and Cairns, J., Jr., eds., 2003, Handbook of ecotoxicology (2d ed.): Boca Raton, Fla., Lewis Publishers, 1290 p.

Hose, J.E., Cross, J.N., Smith, S.G.S., and Diehl, D., 1989, Reproductive impairment in a fish inhabiting a contaminated coastal environment off of southern California: Environemtal Pollution, v. 57, p. 139.

Kime, D.E., 1998, Endocrine disruption in fish: Boston, Kluwer Academic Publishers, 396 p.

Lee, D.S., Gilbert, C.R., Hocutt, C.H., Jenkins, R.E., McAllister, D.E., and Stauffer, J.R., Jr., 1980, Atlas of North American fresh water fishes: North Carolina Biological Survey, Publication no. 1980-12, p. 152 and 608.

Mylonas, C.C., Scott, A.P., Vermeirssen, E.L., and Zohar, Y., 1997, Changes in plasma gonadotropin II and sex steroid hormones, and sperm production of striped bass after treatment with controlled release gonadotropinreleasing hormone agonist-delivery systems: Biology of Reproduction, v. 57, p. 669-675. 
Norris, D.O., and Jones, R.E., 1987, Hormones and reproduction in fishes, amphibians and reptiles: New York, Plenum Press.

Palmer, B.D., and Selcer, K.W., 1996, Vitellogenin as a biomarker for xenobiotic estrogens: A review, in Bengtson, D.A., and Henshel, D.S., eds., Environmental Toxicology and Risk Assessment: American Society for Testing and Materials, STP 1306, v. 5, p. 3-22.

Panek, F.M., 1987, Biology and ecology of carp, in Cooper, E.L., ed., Carp in North America: Bethesda, Md., American Fisheries Society, p. 1-15.

Purdom, C.E., Hardiman, P.A.., Bye, V.J., Eno, N.C., Tyler, C.R., and Sumpter, J.P., 1994, Estrogenic effects of effluents from sewage treatment works: Chemistry and Ecology, v. 8, p. 275-285.

Schmitt, C.J., ed., 2002, Biomonitoring of environmental status and trends (BEST) program: Environmental contaminants and their effects on fish in the Mississippi River Basin. U.S. Geological Survey Open-File Report 20020004, $241 \mathrm{p}$.

Schmitt, C.J., Blazer, V.S., Dethloff, G.M., Tillitt, D.E., Gross, T.S, Bryant, Jr., W.L., DeWeese, L.R., Smith, S.B., Goede, R.W., Bartish, T.M., and Kubiak T.J., 1999, Biomonitoring of Environmental Status and Trends (BEST) Program: Field procedures for assessing the exposure of fish to environmental contaminants: U.S. Geological Survey, Information and Technology Report, USGS/BRD 19990007, 35 p., plus apps.

Schmitt, C.J., and Dethloff, G.M., eds., 2000, Biomonitoring of Environmental Status and Trends (BEST) program: Selected methods for monitoring contaminants and their effects in aquatic ecosystems: U.S. Geological Survey, Information and Technology Report, USGS/BRD 20000005, $81 \mathrm{p}$.

Smith, S.B., Donahue, A.P., Lipkin, R., Blazer, V.S., Schmitt, C.J., and Goede, R.W., 2002a, Illustrated field guide for assessing external and internal anomalies in fish: U.S. Geological Survey, Information and Technology Report, USGS/BRD 2002-0007, 46 p.

Smith, S.B., Gross, and T.S., Denslow, N.D., 2002b, Endocrine biomarkers in largemouth bass (Micropterus salmoides) related to polychlorinated biphenyls (PCBs) in Woods Pond, Housatonic River, Massachusetts: U.S. Geological Survey Open-File Report 2002-001.

Stahl, R.G, Jr., and Clark, J.R., 1998, Uncertainties in the risk assessment of endocrine-modulating substances in wildlife, in Kendall, R., Dickerson, R., Geisy, J., and Suk, W., eds., Principles and processes for evaluating endocrine disruption in wildlife: Pensacola, Fla., Society of Environmental Toxicology and Chemistry, p. 431-448.

Sumpter, J.P., and Jobling, S., 1995, Vitellogenin as a biomarker for estrogenic contamination of the aquatic environment: Environmental Health Perspective, v. 103, p. 173-178.

Tillitt, D.E., Ankley, G.T., Giesy, J.P., Ludwig, J.P., KuritaMatsuba, H., Wesehloh, D.V., Ross, P.S., Bishop, C.A., Sileo, L., Stromborg, K.L., Larson, J., and Kubiak, T.J., 1992, Polychlorinated biphenyl residues and egg mortality in double-crested cormorants from the Great Lakes: Environmental Toxicology and Chemistry, v. 11, no. 9, p. 1281-1288. 